\title{
The Organization of Behavior
}

\section{A NEUROPSYCHOLOGICAL THEORY}

\author{
D. O. HEBB
}

McGull Unioersity

1949

New York - JOHN WILEY \& SONS, Inc. London - CHAPMAN \& HALL, Limited 
COPYRIGET, IY4Y

$\mathbf{B Y}$

Joms WIIEY \& Sons, Inc.

All Rights Roserned

Thas book or any part thereof mast not be reproduced in any form whous the pirtlan permassron of the publasher. 


\section{To \\ G. C. H., C. O. H. and \\ A. $\mathbf{M}$. $\mathbf{H}$.}




\section{Preface}

In this book I have tried to bring together a number of different lines of research, in a general theory of behavior that attempts to bridge the gap between neurophysiology and psychology, as well as that between laboratory psychology and the problems of the clinic

The book is wntten, consequently, with the hope that it will be intelligible to the clmician and the physiologist as well as to psychologists The development of psychological theory can be a cooperative affarr, and has often been so in the past witness Helmholtz, Jackson, Pavlov, Freud. The clnician and the physiologist frequently have direct access to data of first importance for psychology, sometmes without recognizing the fact. Though I have done my best, it may be chimerical to hope that my discussion is extensive and clear enough to stand on its own feet, for the nonpsychological reader. The reader who needs it will find more of the detals of psychological theory in Morgan (1943) on physiological psychology, Hilgard and Marquis (1940) on the theory of learning, Woodworth (1938) on "experimental" (normal hurnan adult) psychology, and Moss (1942) or Maier and Schneurla (1935) on anumal psychology. Of these, Morgan is most durectly relevant, and in several places I have assumed a knowledge of fact to the extent provided by hus text.

It is a pleasure to record my indebtedness to the colleagues who have read and improved the contents of this book. I owe much to students in a semmar at Harvard University in the summer of 1947, and in another at McGill University in the following winter. Part or all of the manuscript was read by Professor Harlow W. Ades, Professor Frank A Beach, Dr J. G Beebe-Center, Professor R B MacLeod, Dr Francis McNaughton, Dr G A Miller, Dr Karl Pribram, Professor H. E Rosvold, and Professor R W. Sperry, and their help is gratefully acknowl- 
edged I am much mdebted to Professor Edwin G Boring, Professor George Clark, Professor K S Lashley, and Dr J C R Licklider for their painstaking and detalled criticism of a large part of the manuscript, improving both matter and style My greatest debt, perhaps, is to the weekly colloquuum and the persistent theoretical debate at the Yerkes Laboratones of Primate Bıology between 1942 and 1947, and to a small group takung part therenn who have also read the entire manuscript and have contributed greatly to it-Professor Henry W Nissen, Mr and Mrs Robt Blum, and Dr. Austin Riesen This board of critics should also include my wrfe, and to it, for the positive contributrons made as well as for some of the changes and deletions forced on me, both the reader and I owe much.

Finally I should like to express my gratitude to those who have helped with clencal work in the preparation of this book, in its various stages. particularly, Alice Sellers, Therese Mahoney, and Margaret Hallıday The McGll Unıversity Research Fund supported some of the expenmental work that is briefly reported here, and also made it possible to secure additional clerical assistance

Bfontreal

D O. Heas

Aprll, 1949 


\section{Contents}

INTRODUCTION

1 THE PAOBLEM AND THE LINE OF ATTACK 1

2 SUMMATION AND LEARNING IN PERCEPTION 17

S FIELD THEORY AND EQUIPOTENTIALITY 38

4 THE FIRST STAGE OF PERCEPTION GROWTH OF THE $\begin{array}{lll}\text { ASSEMBLY } & 0\end{array}$

5 PERCEPTION OF A COMPLEX. THE PHASE SEQUENCE 79

6 DEVELOPMENT OF THE LEARNING CAPACITY IOT

7 HIGHER AND LOWER PROCESSES RELATED TO LEARNING 140

8 PROBLEMS OF MOTIVATION- PAIN AND HUNGER 171

9 THE PROBLEM OF MOTIVATIONAL DRIFT 207

10 EMOTIONAL DISTURBANCES 235

11 THE GROWTH AND DECLINE OF INTELLIGENCE 275 $\begin{array}{ll}\text { BEBLIOGRAPHY } & 305\end{array}$

INDEX • $\quad 321$ 


\section{Introduction}

It might be argued that the task of the psychologist, the task of understanding behavior and reducing the vagaries of human thought to a mechanical process of cause and effect, is a more difficult one than that of any other scientist Certainly the problem is enormously complex, and though it could also be argued that the progress made by psychology in the century following the death of James Mill, with his crude theory of association, is an achevement scarcely less than that of the physical sciences in the same period, it is nevertheless true that psychological theory is still in its infancy There is a long way to go before we can speak of understanding the principles of behavior to the degree that we undetstand the principles of chemical reaction

In an undertaking of such difficulty, the psychologist presumably must seek help wherever he can find it. There have been an increasing number of attempts to develop new mathematical methods of analysis With these, in general, I do not attempt to deal The method of factor analysis developed by Spearman (1927) and greatly elaborated by Thurstone (1935) is well established as a powerful tool for handling certain kinds of data, though the range of its use has been limited by dependence on tests that can be conveniently given to large groups of subjects Another method is the application of mathematics more directly to the interaction of populations of neurons, by Rashevsky, Pitts, Householder, Landahl, McCulloch, and others * Bishop (1946) has discussed the work from the point of new of neurophysiology, and his remarks are fully concurred with here. The prelimmary studies made with this method so far have been obliged to sumplify the psychological problem almost out of

- Two papers by Culbertson (Bull. Math Blophys, 1948, 10, 31-40 and 97-102), and Bishop's review article, list some of the more important of the actual titles in thes field. 
exastence. This is not a criticsm, sunce the attempt is to develop methods that can later be extended to deal with more complex data, but as matters stand at present one must wait for further results before being sure that the attempt will succeed. Undoubtedly there is great potential value in such work, and if the right set of mitral assumptions can be found it will presumably become, like factor analysis, a powerful ally of other methods of study

However, psychology has an intmate relation with the other biological sciences, and may also look for help there There is a considerable overlap between the problems of psychology and those of neurophysiology, hence the possiblity (or necessity) of reciprocal assistance. The first object of this book is to present a theory of behavior for the consideration of psychologists, but another is to seek a common ground with the anatomist, physiologist, and neurologist, to show them how psychological theory relates to their problems and at the same tume to make it more possible for them to contribute to that theory

Psychology is no more static than any other science Physiologists and clunicians who wish to get a theoretical orientation cannot depend only on the writungs of Pavlov or Freud. These were great men, and they have contrubuted greatly to psychological thought. But their contribution was rather in formulating and developing problems than in providing final answers. Pavlov himself seems to have thought of his theory of conditoned reflexes as something in continual need of revision, and experimental results have continued to make revisions necessary. the theory, that is, is stull developing Again, if one were to regard Freud's theory as needing change only in its details, the main value of his work would be stultified Theorizing at this stage is like skating on thin ice-keep movng, or drown Ego, Id, and Superego are conceptions that help one to see and state important facts of behavior, but they are also dangerously easy to treat as ghostly realities, as anthropomorphic agents that want this or disapprove of that, overcoming one another by force or guile, and punishing or being punished Freud has left us the task of developing these provisional formulations of his to the point where such a danger no longer exists When theory becomes static it is apt to become dogma; and psychological 
theory has the further danger, as long as so many of its problems are unresolved, of inviting a relapse into the vitalism and indeterminism of traditional thought.

It is only too easy, no matter what formal theory of behavior one espouses, to entertain a concealed mysticism in one's thinking about that large segment of behavior which theory does not handle adequately To deal with behavior at present, one must oversimplify The risk, on the one hand, is of forgetting that one has oversimplified the problem, one may forget or even deny those inconvenient facts that one's theory does not subsume. On the other hand is the risk of accepting the weak-kneed discouragement of the vitalist, of being content to show that existing theories are imperfect without seeking to improve them. We can take for granted that any theory of behavior at present must be madequate and incomplete. But it is never enough to say, because we have not yet found out how to reduce behanor to the control of the brain, that no one in the future will be able to do so.

Modern psychology takes completely for granted that behavor and neural function are perfectly correlated, that one is completely caused by the other. There is no separate soul or lifeforce to stick a finger into the brain now and then and make neural cells do what they would not otherwise Actually, of course, this is a working assumption only-as long as there are unexplained aspects of behavior. It is quite conceivable that some day the assumption will have to be rejected. But it is important also to see that we have not reached that day yet. the working assumption is a necessary one, and there is no real evidence opposed to it. Our fallure to solve a problem_so far does not make it insoluble One cannot logically be a determinist in physics and chemistry and biology, and a mystic in psychology

All one can know about another's feelngs and awarenesses is an inference from what he does-from his muscular contractions and glandular secretions. These observable events are determined by electrical and chemical events in nerve cells If one is to be consistent, there is no room here for a mysterious agent that is defined as not physical and yet has physical effects (especially since many of the entutes of physics are known only 
through their effects). "Mind" can only be regarded, for scientific purposes, as the activity of the brain, and this should be mystery enough for anyone. besides the appalling number of cells (some nine bllion, according to Herrick) and even more appalling number of possible connections between them, the matter out of which cells are made is being itself reduced by the physicist to something quite unlike the inert stick or stone with which mind is traditionally contrasted. After all, it is that contrast that is at the bottom of the vitalist's objection to a mechanistic biology, and the contrast has lost its force (Herrick, 1929) The mystc might well concentrate on the electron and let behavior alone. A philosophical parallelısm or ıdealısm, whatever one may think of such conceptions on other grounds, is quite consistent with the scientific method, but interactionsm seems not to be.

Psychologist and neurophysiologist thus chart the same bayworkng perhaps from opposite shores, sometimes overlapping and duplicating one another, but using some of the same fixed points and continually with the opportunity of contributing to each other's results The problem of understanding behavior is the problem of understanding the total action of the nervous system, and vice versa This has not always been a welcome proposition, either to psychologist or to physiologist

A vigorous movement has appeared both in psychology and psychiatry to be nd of "physiologizing," that 1s, to stop using physiological hypotheses This pount of vew has been clearly and effectively put by Skunner (1938), and it does not by any means represent a relapse into vitalism The argument is related to modern positivism, emphasizes a method of correlating observable stmull with observable response, and, recognizing that "explanation" is ultumately a statement of relationships between observed phenomena, proposes to go to the heart of the matter and have psychology confine itself to such statements now This point of view has been criticized by Pratt (1939) and Kohler (1940) The present book is written in profound disagreement with such a program for psychology. Disagreement is on the grounds that this arises from a misconception of the scientific method as it operates in the earher stages Those apparently nave features of older scientufic thought may have had 
more to do with hitting on fertile assumptions and hypotheses than seems necessary in retrospect. The ant1-physiological position, thus, in urging that psychology proceed now as it may be able to proceed when it is more highly developed, seems to be in short a counsel of perfection, disregarding the limitations of the human intellect. However, it is logically defensible and may yet show by its fertility of results that it is indeed the proper approach to achieving prediction and control of behavior

If some psychologists jlb at the physiologist for a bedfellow, many physiologists agree with them heartily One must sympathize with those who want nothing of the psychologist's hairsplitting or the indefiniteness of psychological theory There is much more certainty in the study of the electrical activity of a well-defined tract in the brain The only question is whether a physiology of the human brain as a whole can be achieved by such studies alone One can discover the properties of its various parts more or less in isolation, but it is a truism by now that the part may have propertes that are not evident in wolation, and these are to be discovered only by study of the whole intact brain The method then calls for learning as much as one can about what the parts of the brain do (primarly the physiologist's field), and relating behavior as far as possible to this knowledge (primarily for the psychologist), then seeing what further information is to be had about how the total brain works, from the discrepancy between (1) actual behavior and (2) the behavior that would be predicted from adding up what is known about the action of the various parts.

This does not make the psychologist a physiologist, for precisely the same reason that the physiologist need not become a cytologist or biochemist, though he is intimately concerned with the information that cytology and biochemistry provide. The difficulties of finding order in behavior are great enough to requre all one's 'attention, and the psychologist is interested in physiology to the extent that it contributes to his own task

The great argument of the positivists who object to "physiologizing" is that physiology has not helped psychological theory. But, even if this is true (there is some basis for denying it), one has to add the words so far. There has been a great access of knowledge in neurophysiology snce the twenties. The work of 
Berger, Dusser de Barenne, and Lorente de No (as examples) has a profound effect on the physiological conceptions utılized by psychology, and psychology has not yet assimilated these results fully

The central problem with which we must find'a way to deal can be put in two different ways. Psychologically, it is the problem of thought some sort of process that is not fully controlled by environmental stimulation and yet cooperates closely with that stimulation. From another point of vew, physiologically, the problem is that of the transmission of excitation from sensory to motor cortex. Thus statement may not be as much oversimplified as it seems, especially when one recognizes that the "transmission" may be a very complex process indeed, with a considerable tume lag between sensory stimulation and the final motor response The fallure of psychology to handle thought adequately (or the farlure of neurophysiology to tell us how to conceive of cortical transmission) has been the essential weakness of modern psychological theory and the reason for persistent difficultues in dealing with a wide range of expernmental and clinical data, as the following chapters will try to show, from the data of perception and learning to those of hunger, sleep, and neurosis.

In mammals even as low as the rat it has turned out to be impossible to describe behavior as an interaction durectly between sensory and motor processes. Something like thinking, that is, intervenes "Thought" undoubtedly has the connotation of a human degree of complexty in cerebral function and may mean too much to be applied to lower anmals But even in the rat there is evidence that behavior is not completely controlled by immediate sensory events: there are central processes operating also

What is the nature of such relatively autonomous activities in the cerebrum? Not even a tentative answer $15^{\circ}$ avalable. We know a good deal about the afferent pathways to the cortex, about the efferent pathways from it, and about many structures linking the two. But the links are complex, and we know practically nothing about what goes on between the arrival of an excitation at a sensory projection area and its later departure from the motor area of the cortex Psychology has had to find, 
in hypothesis, a way of bridging this gap in its physiological foundation In general the bndge can be described as some comparatuvely simple formula of cortical transmission * The particular formula chosen mainly determines the nature of the psychological theory that results, and the need of choosing is the major source of theoretical schism

Two kinds of formula have been used, leading at two extremes to (1) switchboard theory, and senson-motor connections, and (2) field theory (E1ther of these terms may be regarded as opprobrium, they are not so used here ) (1) In the first type of theory, at one extreme, cells in the sensory system acquire connections with cells in the motor system, the function of the cortex is that of a telephone exchange Connections rigidly determine what anmal or human being does, and ther acquisition constitutes learning Current forms of the theory tend to be vaguer than formerly, because of effective criticism of the theory in its earlier and simpler forms, but the fundamental idea is still maintained (2) Theory at the opposite extreme denies that learnung depends on connections at all, and attempts to utilize instead the field conception that physics has found so useful. The cortex is regarded as made up of so many cells that it can be treated as a statistically homogeneous medrum The sensory control of motor centers depends, accordingly, on the distribution of the sensory excitation and on ratios of excitation, not on locus or the action of any specific cells.

Despite their differences, however, both theoretical approaches seem to imply a prompt transmission of sensory excitation to the motor side, if only by fallng to specify that this is not so No one, at any rate, has made any senous attempt to elaborate ideas of a central neural mechanism to account for the delay, between stumulation and response, that seems so characteristic of thought. There have indeed been neural theories of "motor" thought, but they amount essentially to a contunual interplay of propnoception and munumal muscular action, and do not provide for any prolonged sequence of intracerebral events as such.

But the recalcitrant data of animal behavior have been draw-

- The sumplicity possibly accounts for the opinion expressed by an anatomist who claimed that psychologists think of the brain as having all the finer structure of a bowiful of parridge. 
Ing attention more and more insistently to the need of some better account of central processes This is what Morgan (1943) has recognized in saying that "mental" variables, repeatedly thrown out because there was no place for them in a stumulusresponse psychology, repeatedly find their way back in again in one form or another The image has been a forbidden notion for twenty years, particularly in anumal psychology, but the fiend was hardly exorcused before "expectancy" had appeared instead. What is the neural basis of expectancy, or of attention, or interest? Older theory could use these words freely, for it made no serious attempt to avoid an interactionist phulosophy In modern psychology such terms are an embarrassment; they cannot be escaped if one is to give a full account of behavior, but they still have the smell of animism. and must have, until a theory of thought is developed to show how "expectancy" or the like can be a physiologically intelligible process

In the chapters that follow this introduction I have tried to lay a foundation for such a theory It is, on the one hand and from the physiologist's point of view, quite speculative. On the other hand, it acheves some synthesis of psychological knowledge, and it attempts to hold as strictly as possible to the psychological evidence in those long stretches where the guidance of anatomy and physiology is lacking The desideratum is a conceptual tool for dealing with expectancy, attention, and so on, and with a temporally organized intracerebral process. But this would have Iittle value if it did not also comprise the man facts of perception, and of learning To achieve something of the kind, the limitations of a schema are accepted with the purpose of developing certain conceptions of netral action This is attempted in Chapters 4 and 5, Chapters 1 to 3 try to clear the ground for this undertaking From Chapter 6 onward the conceptions denved from schematizing are applied to the problems of learning, volition, emotion, hunger, and so on. (In general, the reader may regard Chapters 1 to 5 as mainly preparatory, unless he is particularly interested in the neurological detals, or in the treatment of perception, to get the gist of the theory that is presented here one should read the two following paragraphs, and turn directly to Chapter 6 ) In outline, the conceptual structure is as follows: 
Any frequently repeated, particular stimulation will lead to the slow development of a "cell-assembly," a diffuse structure comprising cells in the cortex and diencephalon (and also, perhaps, in the basal ganglia of the cerebrum), capable of actung briefly as a closed system, deliverng facilitation to other such systems and usually having a specific motor faciltation A senes of such events constitutes a "phase sequence"-the thought process Each assembly action may be aroused by a precedng assembly, by a sensory event, or-normally-by both The central faculitation from one of these activities on the next is the prototype of "attention" The theory proposes that in this central facilitation, and its varied relationshup to sensory processes, lies the answer to an issue that is made inescapable by Humphrey's (1940) penetrating review of the problem of the direction of thought.

The kind of cortical organization discussed in the preceding paragraph is what is regarded as essental to adult wakung behavior It is proposed also that there is an alternate, "intrinsic" organzzation, occurring in sleep and in infancy, which consists of hypersynchrony in the firng of cortical cells But besides these two forms of cortical organization there may be disorganization It is assumed that the assembly depends completely on a very delicate timing which might be disturbed by metabolic changes as well as by sensory events that do not accord with the pre-existent central process When this is transient, it is called emotional disturbance, when chronic, neurosis or psychosis

The theory is evidently a form of connectionism, one of the switchboard vanety, though it does not deal in direct connections between afferent and efferent pathways not an "S-R" psychology, if $\mathrm{R}$ means a muscular response The connections serve rather to establish autonomous central actuvities, which then are the basis of further learning In accordance with modern physiological udeas, the theory also utilizes local field processes and gradients, followng the lead particularly of Marshall and Talbot (1942) It does not, further, make any single nerve cell or pathway essential to any habit or perception Modern physiology has presented psychology with new opportunities for the synthesis of divergent theornes and prevously unrelated data, and it is my intent to take such advantage of these opportunltes as I can. 


\section{The Problem and the Line of Attack}

This book presents a theory of behavior that is based as far as possible on the physiology of the nervous system, and makes a sedulous attempt to find some community of neurological and psychological conceptions.

The purpose of this first chapter is to define the major diffcultzes in the way of such an undertaking and to show how these difficulties determine the line it must take. The great need, as I have tried to make clear in the Introduction, is for a physiological theory of thought (or some equivalent term that can be applied to anmals as well as to man).

Let us look first at the specific problem from which the present speculations began, to see why the discussion is oriented as it is

The problem lay in certann puzzling effects of operation on the human brain The effect of a clearcut removal of cortex outside the speech area is often astonishingly small, at times no effect whatever can be found (Hebb, 1942a, 1945b). It is possible that there is always a loss of intelligence in aphasia, when the "speech area" is seriously damaged, but this does not, of course, explain why damage elsewhere should have no effect. It would be unreasonable to suppose that most of the cortex has nothing to do with intelligence, and there are in fact definite indications that this is not true Intelligence must be affected by any large brain injury-yet sometmes it seems not to be.

A final touch is added to the puzzle when we find that it is tests of the Binet type that least show the effect of injunes outside the speech area. The Binet is the measure of intelligence that is most sensitive and accurate with normal subjects How can it be possible for a man to have an IQ of 160 or higher, 
after a prefrontal lobe has been removed (Hebb, 1939), or for a woman (described by Rowe, 1937) to have an IQ of 115, a better score than two-thurds of the normal population could make, after losing the enture right half of the cortex?

Those two are perhaps the most stnkng cases, but high scores after brain operation have been reported by nearly everyone who has used standard tests as a method of study, and they have to be explained. Most investigators have preferred to forget them, and to search only for intellectual defects The defects certanly must exist, and it is important to find them, but it is just as important to learn why Bunet-type tests, the most walid and sensitive indices of normal ability, should often show no effect of injury to the brain

The only explanation that has been proposed, and perhaps the only feasible one, has to do with perceptual learning and with concepts as distunct fróm conditioned rèsponses or motor learnung (Hebb, 1942a). The explanation, roughly, is thus. The level of intelligence-test performance is a function of the concepts a patient has already developed. Once developed, a concept is retained, despite brain damage that, if th had occurred earler, would have prevented the development The patient with bram unjury at maturity may continue to thunk and solve problems normally (in familar fields), although his intelligence would have been far from normal if a simular anjury had happened at buth. The explanation meets the clinical facts and, moreover, is supported by the way in which some intellectual capacites are retained in old age when others are disappearing.

But now we come to the crux of the matter As an explanation, this is only a good start. What is a concept, physiologically and d propos of the loss of neural cells? Though it has been tned before now, an explanation cannot be half neural anatomy and balf consciousness. They are different levels in a logical hierarchy. A problem recognized at one level can be solved by recourse to a more fundamental set of conceptions, but an hypothesis cannot well comprise two levels, take in two universes of discourse at the same tume We want to explain certain clinical facts To really do so, we must find an anatomical and physiological understanding of what is known psy- 


\section{Rejecting Sensory Control}

chologically as a concept, and we must be able to deal with its relation to perception and to learning.

And with that, we land nght in the middle of the generalized problem of explaining mammalian behavior. What is a con-cept, if it is not a conditioned response? What is perceptual learning? And so on. Before such questions can be answered, psychological theory must have a new base of operations As an illustration, a particular problem has been cited, but the difficulty is really general, as the following section will ty to show, and there is no phase of psychological theory in which the same central weakness does not appear

It has already been suggested that the essential need is to find out how to handle thought, and related processes, more adequately The difficulty in doing so goes back to fundamental assumptions If this discussion is to rest on solid ground, it must start with certain ideas with which every psychologist is concerned but which have been confused, vague, or ill defined.

This first chapter, accordingly, will dot some is and cross some $t$ 's, so that we can get down to business in what follows. There are two fundamental assumptions to be dealt with One of them must be rejected, one accepted The rest of this chapter will show how they determine a point of attack for the revision of theory

\section{REJECTING THE ASSUMPTION OF A COMPLETE SENSORY CONTROL}

The first to be discussed is what can be called the assumption of a sensory dominance of behavior It is the idea that behavior is a series of reactions (mstead of actions), each of which is determined by the immediately preceding events in the sensory systems The idea is not altogether consistent with recognuzing the existence of set, attitude, or attention, and an implicit inconsistency of this sort is at the root of the current confusion in psychological theory

It may be noted in passing that the assumption of a sensory dommance of behavior is not the property of any particular theory Theories differ as to how a sensory event has its effect, but not as to its all-mportant role. Gestalt psychology avoids 
words like "stimulus" and "sensation" but as a system is not less preoccupied with stumulus configurations than other systems are with their stimuli (p. 23)

Now for the source of confusion

In the simplest terms, "attention" refers to a selectivity of response Man or anumal is contunuously responding to some events in the environment, and not to others that could be responded to (or "noticed") just as well When an experimental result makes it necessary to refer to "set" or "attention," the reference means, precisely, that the activity that controls the form, speed, strength, or duration of response is not the immediately preceding excitation of receptor cells alone. The fact that a response is not so controlled may be hard to explain, theoretically, but it is not mystical, and "attention" is not necessarily anthropomorphic, or animistic, or undefinable.

Now the tradition in psychology has long been a search for the property of the stimulus which by itself determmes the ensuing response, at any given stage of learning. This approach seems partly a consequence of psychology's persistent fight against animism ${ }^{\circ}$ and deserves respect for that reason, but it is no longer satisfactory as theory Almost without exception psychologists have recognized the existence of the selective central factor that reinforces now one response, now another. The problem is to carry out to its logical conclusion an incomplete line of thought that starts out preoccupied with stimulus or stumulus configuration as the source and control of action, eventually runs into the facts of attention and so on, and then simply

- And partly, as we shall see, the product of antiquated physiological conceptions Fighting anmism meant that psychological phenomene had to be reduced to a pattern of cause and effect The only means at hand was a physiology of the nervous system in which a knowledge of sense organs and penpheral nerve was the main content As a result, it is still easy to feel that psychology becomes part of a larger demonology with any retreat from the stumulus-response formula. As for "insight," "purpose," "attention"any one of these may still be an invocation of the devil, to the occasional psychologist. However, this attitude should not be too much made fun of It cannot be emphasized too strongly that there is continual danger of slipping momentarily into anmistre thinking, and consistent use of the S-R formula does at least avoid that danger effectively It behooves those of us who wish to use other terms to see that they are clearly defined 


\section{Rejecting Sensory Control}

agrees that attention is an important fact, without recognizing that this is mconsistent with one's earlier assumptions To complete this process, we must go back and make a change in the basis of the theory

There are three points here one is that psychologists have generally recognuzed the existence of attention or the like, another that they have done so reluctantly and sparingly, and have never recognized the fact in setting up theories. The third point is obvious enough, that we need to find some way of dealing with the facts consistently Since everyone knows that attention and set exist, we had better get the skeleton out of the closet and see what can be done with it.

The first two of these points have been pretty clearly established by Gibson (1941) His revew needs some clarification in one respect, since he declined to attempt any definition of "set" or any other of the long list of terms with a similar meaning that he gathered together, although he evidently recognized, in classifying them so, that they have something in common This common meaning has already been defined. When one considers the problem in the light of the implicit assumption of a sensory dominance of behavior it becomes clear at once that the notions of set, attention, attitude, expectancy, hypothesis, intention, vector, need, perseveration, and preoccupation (Gibson, 1941, pp 781-782) have a common element, and one only. That element is the recognition that responses are determined by something else besides the immedrately preceding sensory stimulation. It does not deny the importance of the immediate stimulus, it does deny that sensory stumulation is everything in behavior.

All such terms, then, are a reference to the "central process which seems relatively independent of afferent stumul," defined by Hilgard and Marquis (1940), which I shall call here the autonomous central process Gibson's revew shows in effect that the process is ubiquitous, that it crops up in every sort of psychological investıgation-and that almost everyone has recognuzed its existence, in one form or another To Gibson's list can be added Pavlov's (1928) and Hull's (1943) stimulus tracea lasting cerebral state, set up it is true by a specific stimulus but not transmitted and dissipated at once, Beach's (1942) cen- 
tral excitatory mechanism, Morgan's (1943) central motuve state, and Kleitman's (1939) "interest"-a factor in wakefulness. All these things have the same property of an activity that has a selective effect on behavior without being part of the present afferent excitation.

Everyone has had such ideas about the control of behavior, and yet, as Gibson noted, "The meaning [of the term "set"] is felt to be unsatusfactory, and the concept is employed reluctantly and only because the facts make it absolutely unavoidable" The reluctance is partly no doubt because of a feeling that the concept is animstic, in some obscure way But why animism, If the facts of behavior make it unavoidable? The trouble really seems to have been in finding how to make an essential idea intelligible

Hilgard and Marquis' central process, and Beach's central excitatory mechanism, are hypothetical entities, but they certainly have no flavor of animism about them "Attention" and "set" are now seen to fall in the same class it may well be that their connotations are misleading and that we shall have to look for new terms, but the idea itself is respectable, and such language need no longer risk starting a witchhunt.

\section{THE NEUROLOGICAL PROBLEM OF ATTENTION}

There is a further hazard on the course This is the apparent lack of a theoretical rationale for the autonomous central process Actually, modern neurophysiology has already removed this difficulty.

Here again the situation can only be understood historically A main function of the neural cell is of course to transmit exc1tations, and earlier ideas of anatomy and physiology made the central nervous system appear, in principle, a collection of routes, some longer, some shorter, leading without reversal from receptors to effectors-a mass of conductors that hes mactive untrl a sense organ is excited, and then conducts the excitation promptly to some muscle or gland We know now that this is not so, but the older idea still has a profound effect on psychological thought-demonstrated, for example, in the assumption of sensory dominance, discussed above 


\section{Neurological Problem of Attention}

The lack of a rationale for nonsensory influences on behavior that seemed to exist in 1920 certainly exists no more Psychologists have long had to recognize (since the days of the Wurzburg school at least) the exustence of a determining tendency, whether physiology made it comprehensible or not. Modern electrophysiology has more than caught up with psychology and now provides abundant evidence to support the same idea When the detailed evidence of neurophysiology and histology are considered, the conclusion becomes inevitable that the nonsensory factor in cerebral acton must be more consistently present and of more dominating importance than reluctant psychological theory has ever recognized Instead of a joker to occasionally confuse the student of behavior, nonsensory activities appear in every fall of the cards and must make up a large share of the deck. Neurophysiologically, it may even become a problem to account for any consistent effect of a specific stimulus (pp 121, 126).

Electrophysiology of the central nervous system indicates in brief that the brain is continuously active, in all its parts, and an afferent excitation must be superimposed on an already existent excitation. It is therefore impossible that the consequence of a sensory event should often be uninfluenced by the pre-existent activity. If we recognize in that activity the psychologically known factor of set and the like (as Denny-Brown, 1932, suggested some tume ago), the problem for psychology is no longer to account for the exastence of set but to find out how it acts and above all to learn how it has the property of a consistent, selective action instead of producing the random-error distribution postulated by Hull (1943) in his "oscillation princople."

So there really is a rational basis for postulating a central neural factor that modifies the action of a stimulus. The theoretical problem now is to discover the rules by which it operates At first glance this is a problem for the neurophysiologist only But look closer, much of the evidence, from which these rules must be worked out, is psychological, or behavioral. The problem is after all the problem of attention, and seen best in the activity of the whole anmal It is $\mathrm{n}$ the highest degree unlukely that it can be solved either from the physiological evi- 
dence alone or from the behavoral evidence alone What we need, evidently, is some synthesis of both kinds of datum The psychological data have been reviewed briefly (for a fuller account, see Hilgard and Marquis, 1940; Gibson, 1941, Morgan, 1943-under such headings as attitude, expectancy, and set). Let us turn now, again briefly, to the anatomical and physiological evidence that has made a drastic change in the theoretical problems of behavior.

For our purposes, the physiological evidence can be treated under two heads, as bearing on (1) the existence and properties of a continuous cerebral activity, and (2) the nature of synaptic transmission in the central nervous system

1 The studies reviewed by Jasper (1937), beginning with Berger's work and its confirmation by Adrian, have shown with practical certainty that the central nervous system is contunuously active, in all its parts, whether exposed to afferent stimulaton or not It appears that the EEG, or electroencephalogram, must be at least 'm part a summation of action potentials, an Index of actual cellular firing There are changes of cell potential without active transmission of impulses (Gibbs, 1945), and it is quite possible that these are the source of much of the EEG. However, there is a considerable body of endence to show that neural tissue is persistently active, and presumably the EEG includes a record of that activity It is taken here as a working assumption that the EEG is correlated with neural firing-that large potentrals indicate a local synchrony of firng, even though other factors contribute to the size of the potentral The psychological usefulness of this assumption will perhaps become evident in the following chapters.

The evidence concerning persistent activity consists first of a number of studies showing directly that the neural cell fires spontaneously, $i e$, under no stimulation except that of the nutrient fluids bething it (Fessard, cited by Jasper, 1937, Prosser, 1934, Lehmann, 1937a, 1937b, Bronk, 1939, Dubner and Gerard, 1939, Libet and Gerard, 1939). Secondly, a very important paper for the theory of behavior, by Weiss (1941a), reports spontaneous, almost incessant motor activity in a transplanted amphubian limb innervated from a pool without afferent fibers. The activity of the limb was not normally coordinated, which 
agrees with the general idea that sensory activity is essental to the regulation of central neural firing but not essential to initiating it. Funally, the work of Adran and his collaborators. Adran and Matthews (1934) rejected the earlier opinion of Adrian (1931) and Adrian and Buytenduk (1931), and concluded defnitely that the EEG is the summation of single sharp potentials, the result of actual cellular firing They concluded further that the activity is not necessanly maintamed by sensory activity Elsewhere Adrian (1934, p. 1126) has stated his general conclusions. "There are cell mechanisms in the brain which are set so that a periodic discharge is bound to take place. The moment at which it occurs can be greatly altered by afferent influences, but it cannot be postponed indefinitely"

Several comments may be made on these conclusions Spontaneity of firing by central neural cells is not philosophic indeterminacy, as some writers have thought, the "spontanesty" means only that synaptic stimulation is not the sole cause of firng. As Adrian pointed out, also, the cell that is capable of firng spontaneously is also open to synaptic control. If it is left undisturbed the processes of metabolism will lead in the end to firng, but obviously an afferent stumulation could always catch the cell in the period between the absolute refractory state and the time of spontaneous discharge With a frequent arrival of mpulses at the synapse, therefore, the cell can never reach the point of spontaneous action but must remain under afferent control

A very interesting relationship exists between sensory activity, EEG, and coordinated, adaptive behavior (or "conscious state"). There 1s, in the first place, Adraan and Matthews' observation that sensory processes, mstead of supporting synchronous, rhythmic firing and large potentials in the EEG, have the opposite effect They introduce irregularity and flattening of the electrical record In the second place, large potentials, or "hypersynchrony," negate or may negate normal function (Jasper, 1941). That is, sensory activity breaks up hypersynchrony and makes for normal, coordinated, adaptuve activity Bartley and Bushop (1933) and Adrian and Matthews have even proposed that anestheties produce "unconsciousness" by suppressing sensory activity rather than actung directly on the cerebrum 
Itself. It does seem clear from the facts discussed, from the large potentials observed in sleep, and from the hypnotic effect of minimizing the normal variation of sensory activity, that the sensory input to the brain has a constantly necessary function, for adaptive behavior We shall return to this point later (p 145). For the present, it has been advisable to point out that recognuzing the existence of spontaneous neural activity is neither indetermmacy nor a denial of the importance of sensory processes in normal behavior.

2 The nature of synaptic transmission in the central nervous system is also of fundamental importance for the theory of behavior There are two radical modifications of earlier ideas. transmission is not simply linear but apparently always involves some closed or recurrent curcurts, and a single impulse cannot ordinarly cross a synapse-two or more must act simultaneously, and two or more afferent fibers must therefore be active in order to excite a third to which they lead

The concepts of neural action chiefly developed by Lorente de No (1938a, 1938b, 1939, 1943) are well enough known by now to need no elaborate revew It is necessary, however, to point out that they have revolutionary implications for psychological theory.

In particular, the psychological criticism advanced by Koffka (1924), Lashley $(1929 b, 1930)$, and Kohler (1929), eganst the theory of neural connections as the basis of learning, applies only to the older theory of linear, sensori-motor connections, in which a single cell was supposed to be always capable of excitIng a second cell with which it synapsed The criticism is effective against the idea that synaptic resistances are all that determines the direction of transmission at the synapse $A$ radical change in the whole problem, however, has been made by considerations of tuming in transmission (Gasser, 1937) and by the conception of "optional transmission" (Lorente de No, 1939)

In a single system, and with a constant set of connections be-, tween neurons in the system, the direction in which an entering excitation will be conducted may be completely dependent on the tuming of other excitations Connections are necessary but may not be decisive in themselves, in a complex system, espe- 
Itself. It does seem clear from the facts discussed, from the large potentials observed in sleep, and from the hypnotic effect of minimizing the normal variation of sensory activity, that the sensory input to the brain has a constantly necessary function, for adaptive behavior We shall return to this point later (p 145). For the present, it has been advisable to point out that recognuzing the existence of spontaneous neural activity is neither indetermmacy nor a denial of the importance of sensory processes in normal behavior.

2 The nature of synaptic transmission in the central nervous system is also of fundamental importance for the theory of behavior There are two radical modifications of earlier ideas. transmission is not simply linear but apparently always involves some closed or recurrent curcurts, and a single impulse cannot ordinarly cross a synapse-two or more must act simultaneously, and two or more afferent fibers must therefore be active in order to excite a third to which they lead

The concepts of neural action chiefly developed by Lorente de No (1938a, 1938b, 1939, 1943) are well enough known by now to need no elaborate revew It is necessary, however, to point out that they have revolutionary implications for psychological theory.

In particular, the psychological criticism advanced by Koffka (1924), Lashley $(1929 b, 1930)$, and Kohler (1929), eganst the theory of neural connections as the basis of learning, applies only to the older theory of linear, sensori-motor connections, in which a single cell was supposed to be always capable of excitIng a second cell with which it synapsed The criticism is effective against the idea that synaptic resistances are all that determines the direction of transmission at the synapse $A$ radical change in the whole problem, however, has been made by considerations of tuming in transmission (Gasser, 1937) and by the conception of "optional transmission" (Lorente de No, 1939)

In a single system, and with a constant set of connections be-, tween neurons in the system, the direction in which an entering excitation will be conducted may be completely dependent on the tuming of other excitations Connections are necessary but may not be decisive in themselves, in a complex system, espe- 
crally, tume factors must always influence the drection of conduction. The older ideas of neural transmission gave synaptic connections too much rigidity, as a determinant of behavior The rigidity has now disappeared, the idea of connections can again be useful in psychological theory, and the question of "synaptic resistances" is completely reopened

Let me now summarize what has been said, in this and the preceding section, about attention, set, attitude, and so on (1) All psychologists have recognized some such factor in behavor It undoubtedly exists (2) Recognuzing it is really a denial that behavior is only a series of responses to environmental stimulation One important meaning of "attention" or the like is the reference to a partly autonomous, or nonsensory, cerebral activity the "autonomous central process" (3) The problem for psychology then is to find conceptions for dealing with such complexities of central neural action conceptions that will be valid physiologically and at the same tume "molar" enough to be useful in the analysis of behavior (4) Psychology is still profoundly mfluenced by the very "molecular" conception of linear transmission through a sequence of single cells The conception is no longer valid physiologically, just as it has long been without psychological usefulness The attack on neural connections as an explanation of behavior was really an attack on this particular conception of the way connections operate, modern neuroanatomy and electrophysiology have changed the question completely, and the significance of synaptic connectons must be examined all over again

Our problem, then, is to find valid "molar" conceptions of neural action (conceptions, $i e$, that can be applied to largescale cortical organizations) Bishop (1946, p. 370) has made the point, in another context, that this is an essential problem for neurophysiology also But psychologists can hardly sit around with hands folded, waitmg for the physiologist to solve it In its essence the problem is psychological and requures a knowledge of the psychological as well as the physiological evdence for its solution 


\section{PERCEPTUAL GENERALIZATION AND THE ASSUMP- TION OF A STRUCTURAL MEMORY TRACE}

Now for a second fundamental assumption of psychological theory. this time, one that must, it seems, be accepted, but in accepting it we must also recognize the difficulties it entals and provide for them These difficulties in fact determine the main features of the theory presented in this monograph

The assumption we must accept is that the memory trace, the basis of learning, is in some way structural and static, and the difficulties in the way of making the assumption are mainly in the facts of perceptual generalization that have been emphaszzed by Gestalt psychologists (Koffka, 1935, Kohler, 1929, 1940) and Lashley $(1938 b, 1942 a)$ The problem raised by these writers is crucial and must be disposed of before we touch anything else.

Lashley has concluded that a Iearned discrimmation is not based on the excitation of any particular neural cells It is supposed to be determined solely by the pattern, or shape, of the sensory excitation Kohler, also stressing the apparent fact that the pattern and not the locus of stimulation is the important thing, has developed a theory of electrical fields in the bram which control cerebral action. Like Lashley, be explicitly denues that the same cells need be excited to arouse the same perception

This suggests that the mnemonic trace, the neural change that is induced by experience and constitutes "memory," is not a change of structure. Other facts, at the same tume, are an even stronger argument that it must be structural. A structural trace, as we shall see in a moment, must be assumed, but when we do so we have to find some way of fitting in the facts of perception.

If it is really unimportant in what tussues a sensory excitation takes place, one finds it hard to understand how repeated sensations can renforce one another, with the lastng effect we call learning or memory It might be supposed that the mnemonic trace is a lasting pattern of reverberatory activity without fixed locus, like some cloud formations or an eddy in a millpond But If so it seems that the multutudinous traces in the small confines 
of the cerebral cortex would interfere with one another, producing a much greater distortion of early memories by later ones than actually occurs

Moreover, violent cortcal storms can occur (as in grand mal epilepsy or cerebral concussion) without a detectable effect on earher memories. That the trace should be purely "dynamic"a pattern of activity not dependent on structural changes for its permanence-thus seems in the highest degree unlikely. No one has explicitly made such an assumption, yet how otherwise are the known properties of a learned discrimination to be accounted for, with its inevitable tendency to be generalized beyond what has already been experienced by the anımal-its apparent undependence of excitation in specific cells?

In addition to the facts of perceptual generalization, two other forms of evidence might make it difficult to postulate a structural trace as the basis of memory One is from Lashley's (1929a) exturpation experiments, showing that the removal of blocks of the rat's cerebral cortex does not affect habits selectively. If one habit is affected, others are also. From this, Lashley has concluded that memory traces are not localized in the cerebral cortex, but himself has pounted out (Lashley, 1929b) another possible interpretation His evidence is consistent with the idea that the trace is structural but diffuse, mvolving, that 1s, a large number of cells widely spaced in the cortex, physiologically but not anatomically unified Thus is not, consequently, cruczal evidence for or against the notion of structural traces in the cortex

The other evidence that seemed once to prevent postulating a structural trace is found in the work of Wulf (cited by Koffka, 1935) and later investgators who have interpreted their studies of human memory for patterns to mean that the trace is spontaneously active, and does not lie dormant or merely deteriorate with the passage of time. Hanawalt (1937), however, effectively critcized the earlier evidence for this idea, and Hebb and Foord (1945), having obtained data inconsistent with Wulf's hypothesis, re-examined the later work that managed to avord Hanawalt's criticism They have shown that there is no evidence to even fauntly support the idea of slow, spontaneous changes in the trace This conception must be abandoned. 


\section{The Problem and the Line of Attack}

Thus the only barrier to assuming that a structural change in specific neural cells is the basis of memory lies in the generalization of the perception of patterns. Man sees a square as a square, whatever its sze, and in almost any setting $A$ rat trained to look for food behind a horzontal rectangle will there-

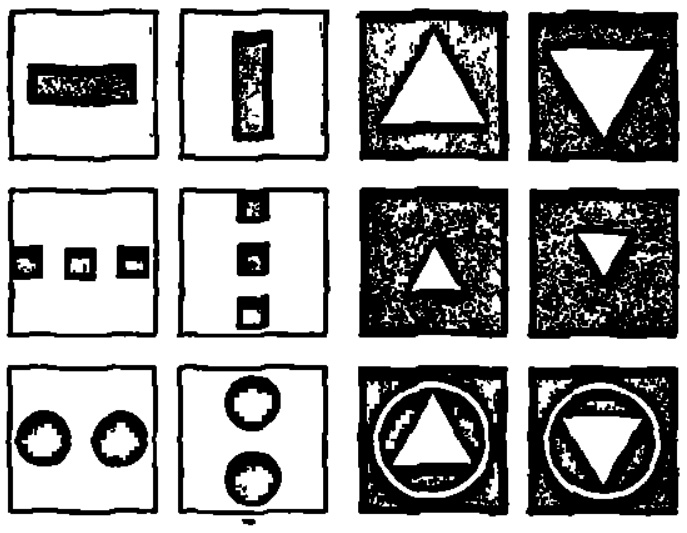

Freurks 1 Illustrating generalization in perception A rat is first trained with the two duagrams at the top, in the left half of the figure, until he always avoids the vertical bar and chooses the horizontal Ho is then tested with the next parr of diagrams, the horizontally and vertically arranged squares, and with the next parr, circles side by side versus curcles one above the other Transfer of response occurs, the rat in each case preferning the horkzontal diagram, showng that perception of horzontal and vertical is generalized beyond any particular pattern. Similarly, after training with the large plain triangles, top nght, the animal is tested with smaller trangles and with circumscribed triangles If the erect thangle, the one to the left, is postive in trauning, the rat will choose the left-hand duagram when presented with etther of the two lower pairs, agam demonstrating perceptual generaluzation

after choose almost any horizontäl figure, such as an interrupted line or a pair of circles side by side (figure 1). Trained to choose a solid upright trangle and to avord an inverted trangle, he will discriminate consistently between outlines of triangles, trangles with confusing figures added (such as circumscribing circles); and trangles of different size, which cannot thus excite the same retinal cells simultaneously (Lashley, I938b) Rats reared in darkness, then trained in the same way, show the same perceptual generalizations (Hebb, 1937a). 
These are concrete, undisputed facts of behavior. They have been interpreted as meaning that perception is independent of the locus of excitation, and this interpretation has been tacitly accepted as inescapable. The result is an awkward dilemma for theory, sunce, as we have seen, it is hard to reconcle an unlocalized afferent process with a structural (and hence localized) mnemonic trace

Lashley's (1942a) hypothesis of interference patterns is the one explicit attempt to solve this difficulty and to deal adequately with both perception and learning. As such it deserves special mention here, although we shall see that in other respects it faces great difficulties

Other writers have had to choose one hom of the dilemma. Kohler (1940), for example, starts out with the facts of perceptual generalization, in his theory of cerebral fields of force, and then cannot deal with learning $\mathrm{He}$ has no apparent way of avoiding a fatal difficulty about the nature of the trace, its locus and structure This is another aspect of the difficulty for Gestalt theory rased by Bonng (1933), who pointed out that at some point the perceptual process must act on specific cells in order to determine a specific response

The theory elaborated by Hull (1943), on the other hand, is to be regarded as providing first of all for the stability of learning It then has persistent difficulty with perception. The princple of "afferent neural interaction" appears to be a concession extorted by the facts of perceptual generalization. With this, there is some danger that the entre system may lose its meaning The great value of Hull's theory is in showing how one may conceive of variable behavior as determined by constant causal relationshups between stumulus, mtervening variables, and response. Thus is brilluantly achreved, for an important segment of behavior. But then the postulate of afferent neural interaction adds that anything may happen when two sensory events occur at the same time-which of course they are always doing. Evidently no prediction is possible until the limits, and the determinants, of afferent neural interaction can be given in detal. This it seems demands that the neurological reference, already present in the theory, be made explicit, and detalled For our present purposes, at any rate, Hull must be regarded as not 
yet havng solved the problem of dealing with the perceptual process in a theory of learming, although it remains possible that his program (Hull, 1945) will do so in the future.

\section{THE MODE OF ATTACK}

How are we to provide for perceptual generalization and the stability of memory, in terms of what the neuron does and what happens at the synapse? We must suppose that the mnemonic trace is a structural change, the difficulty, in supposing it, is a confluct with the idea that only the pattern, and not the locus of sensory stinulation, is important in perception, so let us begn by asking whether that idea is, after all, securely established.

That paragraph puts the theoretical approach of this monograph I propose to go over the evidence on perception again and show that it is not what it seems We do not know that pattern is everything, locus nothing. Next, an alternative set of ideas about perception will be developed. According to these ideas, perception does depend on exciting specific parts of the receptor surface, mnemonic trace and perceptual generalization need no longer be at odds with one another, and a physiological meaning of attention (or set, or expectancy) shows up.

Now let us see, in the following two chapters, what revisions can be made in current ideas about perception so that they can be extended into a more general theory The reader will observe that the discussion deals almost enturely with visual perception This is not because vision has any unique significance, but because it is in visual perception, with few exceptrons, that the problem of patterning and form has been studied experimentally It can be assumed that perception in other sense modes does not depend on fundamentally dufferent prunciples 


\section{Summation and Learning in Perception}

This chapter begins a revision of perceptual theory. The immediate objective is to show that "simple" perceptions are in fact complex that they are additive, that they depend partly on motor actuvity, and that their apparent simplicity is only the end result of a long learning process.

The preceding pages have tred to show that the crucial problem of perception must be dealt with explicitly at the very beginning of a psychological theory, and that one has the choice of two approaches to this problem One must decide whether perception is to depend (1) on the excitation of spectic cells or (2) on a pattern of excltation whose locus is unimportant Current opinion seems tacitly to have accepted the Gestalt argument (and Lashley's argument) that the only tenable assumption is the second of these possibilities.

The theory to be presented here is drametrically opposed to this aspect of Gestalt theory, and is based on assumption 1, that a particular perception depends on the excitation of particular cells at some point in the central nervous system Now the Gestalt argument depends, I believe, on another assumption. that when one perceives a simple figure (such as square or circle) one perceives it directly as a distinctive whole, without need of any learning process and not through a pnor recogns-

- This and the followng chapter may be disregarded by the reader who is not particularly interested in the theory of perception These two chapters are in a sense preventive The theory of behavior presented later would appear unpossible to the reader who is famular with the current literature on perception if he had not been shown that the alternative (and at first sight preferable) approach of "field theory" or "equpotentuality" has very serious difflculties indeed-more serious than has been recognized. 
tion of the several parts of the figure If one makes this assumption-if the perception of a square is as simple and immediate as it seems to us as adults-I believe that the Gestalt argument is unanswerable. But if on the other hand the perception is additive, a senal reconstruction (though very rapid and "unconscious" for the normal adult), the theoretical problem would be very much changed

In this chapter, accordingly, an attempt is made to show that quite simple diagrams are not percerved directly as distinctive wholes-that, though the stimulus has a unitary action in the figure-ground relationship, the perception of identity depends on a series of excitations from the parts of the stumulating diagram If this can be established, it will remove the necessity of accepting field theory, and the following chapter will then attempt to show that field theory actually is not consistent with some of the facts of perception

The work of Senden (1932) and of Riesen (1947) is fundamental to my argument here Senden's monograph is a comp1lation of all published reports on the vision of the congenitally blind given sight by a surgical operation after the patient was old enough to talk to the examuner and describe what he saw In some respects the data are incomplete, but the report is repeatedly referred to here because it contains the only existent evidence concerning the course taken by the early development of human perception At first sight, some of the reported facts are literally almost incredible, since they differ so much from what would be predicted by current theory (either of perception or of learning) There is, however, a considerable unanimity among the writers reviewed by Senden, some of whom evidently were not aware of the reports made by others, and the work of Riesen (1947), who reared chmpanzees in darkness to an age when the nomal chimpanzee makes an effective use of vision, fully confirms Senden's clinical evidence.

The two reports, by Senden and Ruesen, are complementary; and, though many detals are not clear, the human and chimpanzee data taken together seem to requre radical changes in the theory of perception and of learning. 


\section{DISTINCTION OF "PRIMITIVE UNITY" FROM OTHER PROPERTIES OF THE PERCEIVED FIGURE}

As a prelmmary, certain terms must be defined I want to show that simple figures do not always act as wholes, innately. But it is undoubtedly true that they sometimes do so in one respect-in the figure-ground relationship so this property of a perceived figure is to be distingushed from others, in which summation and learning are important Accordingly, the following are distungushed the conceptions of (1) a primitive, sensorily determined unity, (2) a nonsensory unity, affected by expenence, and (3) the identity (also affected by experience), of a perceived figure.

The primitive untty of a figure is defined here as referring to that unity and segregation from the background which seems to be a direct product of the pattern of sensory excitation and the inhented characteristics of the nervous system on which it acts

Rubin (1921) elaborated the conception of the figure-ground relationshup, in a study of visual perception of patterns with clearly marked boundanes In order to elucidate the relation of figure to ground (the relation of the perceived object or surface to other objects and surfaces which make up the background) he put particular stress on "ambiguous" figures (figure 2) This is the special case in which ether of two parts of a diagram may be seen as figure, each alternating as part of the ground when the other is figure The pronciples he established, however, are most fully operative in the unambiguous figure, made up of a homogeneous zone of color surrounded by another color and having a sharply defined boundary-an abrupt transition in brightness from one zone to the other at all points Such a figure may or may not be regular It is as well lllustrated by a splash of ink or by a silhouette of an anmal as by a white crrcle on a black strmulus card.

An area thus sensonly delimited is seen as one, unified and distinct from its surroundings, by any normal person, by the congentally bind on the first occurrence of vision following operation for cataract (Senden, 1932), by the normal rat (Lashley, 1938b), and apparently also at first vision by the rat 
that has been reared in darkness (Hebb, 1937a) The unity and distunctiveness of such figures from therr background, then, is independent of experience, or "primitive"

It is not possible to specify exactly the stimulating conditions which determine the primitive figure-ground organization. I do

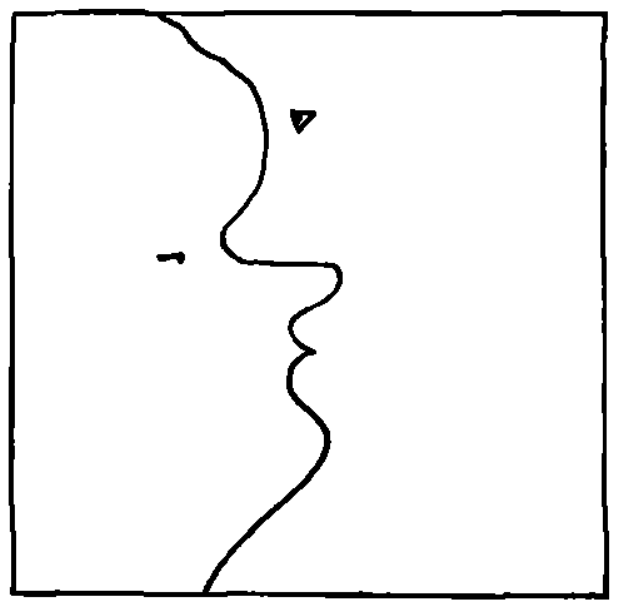

Fieurr 2 Ambiguous figure In this diagram it is possible to see elther of two profiles, but only rarely can the two be seen sunultaneously, they alternate instead When a figure appears on the left, the profile of a rather chubby man, the white area on the left appears as a more soldd surface and one that is nearer to the observer This relationship is reversed when a figure appears on the nght-the profile of one suffenng from gigantc tumors of the neck and of the frontal bone The theoretical importance of such reversals of figure-ground relationshups has been stressed by Gestalt psychologasts, though there is considersble difficulty in subsuming the instabulity under perceptual theory

not exclude as nonprimitive the perception of groupings, nor the segregation of a patch of color which has ill-defined boundaries. There are suggestions in Senden's (1932) monograph and in rat behavior that the perception of such units in the visual field may also be independent of experience Senden's monograph is of the first importance for understanding the perceptual process, but in several respects the evidence is far from clear The earler wnters whose reports are summanzed did not recognize some of the psychological problems to which their 
observations are relevant. What Senden does show, in the fact that patients always responded to certan objects as wholes and could on occasion detect differences between objects even in spite of nystagmus, is that there is a primitive or mnate figureground mechanism. He does not make it possible to state its limiting conditions.

\section{THE "NONSENSORY" FIGURE}

The nonsensory figure-ground organzzation is defined as one in which the boundanes of the figure are not fixed by gradients of luminosity in the visual field It is affected by experience and other nonsensory factors, and is not mevitable in any perception. In contrasting the primitive and the nonsensory figure, however, one need not imply that a perceived figure must be exther of one or the other kind They are rather two extremes, and in most perceptions both sensory and nonsensory factors affect figure-ground organzzation

The one of these extremes, in which exactly the same figural boundanes are recognized by anyone, has already been illustrated. The other is quite common in ordinary experience, although I have not found any discussion that makes its meaning explict. It is implied, for example, by Gibson and Crooks (1938) in their discussion of the perceptual field of the automobile driver, and the shrmking or expanding of a percelved zone of safety from potentral collision. In general terms, the nonsensory figure occurs in perception whenever the subject responds selectively to a limited part of a homogeneous area in the visual field One may look at the "middle part" of a rope as distnct from the rest, in knot-tying, or perceive the "foreground" of an unbroken landscape. It is a commonplace that in human perception many entities exust, like the place in a continuous expanse of lawn where a flowerbed is to be put in, such that no sensory delimitation of figure from ground can even be suggested The "corner" of a room certamly does not always refer to a geometrical point, it is extended, and yet does not comprise all of the two (or three) rectangular surfaces which determine it Its locus is sensorly fixed, but not its boundaries, and lan- 
guage is full of terms with a simlar implication for the theory of perception

Even commoner in everyday perception is the percerved entity in which both sensory and nonsensory factors cooperate Here the figural boundary may follow one of a number of possible luminosity gradients in the field, the particular one that is effective being usually determined by experience in one form or another This is llustrated by Leeper's (1935) experiment, which demonstrated a lasting control, by earler experience, of the particular perception that is made with Boring's ambiguous figure, "My wife and my mother-in-law" (Boring, 1930) Another illustration is the process of slow learning to see a configuration in a particular way, as in the gradual decrease of the difficulty a novice has in following the boundaries of a thalamic nucleus that are obvious to the expert.

There is reason to believe that the rat, as well as man, finds some figure-ground relations obvious and inescapable, and detects others only after prolonged experience. That is, the distinction of primitive and nonsensory figures applies also to the rodent's perception Lashley $(1938 b$, pp 156, 185) points out that success in discrumination and generalization evidently depends on the rat's finding a differentiating characteristic between two figures, or one that is common to a pattern already known and the new one which is presented in the testing situation. It is equally clear that the distmctive part of the test pattern is not obvious to the rat at once, this part, whatever it is, is what determines the response and becomes the true figure (as contrasted with what the experimenter meant to be the figure), the rest merging into ground.

In general, Gestalt writers on the organization of the figure have been concerned to show that it cannot be reduced to experience and learning, and have thus selected cases for discussion in which sensory dynamics alone is enough to produce an effective figure-ground organization They have, that is, concentrated attention on the primitive figure, and they give the impression that the spontaneity of its organization is a property of any figure True, one can find many passages in the literature on Gestalten that refer to figures not sensorly delimited, 


\section{The "Nonsensory" Figure}

but no stress on the fact that this implies some role of learning in the delimitation.

Kohler (1929), for example, has written. "Since 'real form' presupposes a segregated whole, the existence of 'form' depends upon factors of strmulation similar to those upon which the segregation and organization of wholes depend Again, definite relations in the total constellation of retinal stimuli are found to be decisive for the existence of real form" (p 202, my italics draw attention to the stress put on sensory factors) Again, after referring to Gottschald's expeninents, Kohler says "After these results, whoever defends the automatic influence of past expenence upon our seeing definte forms, will have incumbent upon him the task of supporting his theory by other exper1ments If such an influence exists, it must be restricted to rather special cases" (p 208, italics mine).

Attention is drawn to this aspect of Gestalt theory because it helps one to define the point at which one can diverge from the theory without faling to recognize the great contribution it has made to modern psychology, which has been shaped to a great extent by the impact of Gestalt ideas on behaviorism There are few psychologists who would not own a debt of this kind to Gestaltheorie, and few who do not also feel the need of qualifyng the theory in some way, though it is not easy to see just how this is to be done without losing the values that have been abtained with its help

In the pages from which the passage above is taken, Professor Kohler, I believe, provides the necessary clue His argument appears to depend on a complete antithesis of experience and innate sensory dynamics, and it is this antithesis that we may avo1d The question that is asked by Kohler is whether sensory organzation is wholly innate, or wholly experiental." If these are the only two alternatives, the argument is unanswerable Kobler and Koffka and Lashley have unquestionably slan the

- In the monograph of Kohler and Wallach (1944, pp 316, 323) there are also passages that seem not intelligible unless one is thinking in terms of such an extreme dichotomy between completely organzed sensory processes (mnately) and completely unorganuzed ones This does not do justice, either to current learning theory, or to the effectiveness of the earher Cestalt arguments in favor of an innate sensory organization 


\section{Summation and Learning in Perception}

dragon of pure learning theory, in the field of perception, and no one today would argue that perceptual organization is wholly acqured, there is some innate organization But this of course does not show that the organization is entrrely innate There is always a possibulity that perception has a partly innate, partly learned organzzation, and that besides the figure that has a "primitive unity" there are "nonsensory figures" in which experence has an important role

I propose, then, that Gestalt theory made an essential contrrbution when it showed that there are innate factors in percepton, but that it has tended to carry the argument too far in denying that learning and expenence have any mportant role $m$ the perception of simple configurations (apart, of course, from learning the meaning of the configurations) This, as we have seen, has led to emphasis on a dommant role of sensory dynamies, important as sensory processes are, however, they do not completely dominate etther behavior or perception

It is also important to see that the argument aganst an effect of experience on perception often requires the assumption that any perceived figure is perceived as a whole, in all respects Thus the upper diagram of figure 3 has been regarded as quite unfamliar to the observer (Kohler, 1929). Subjects shown this diagram for the first time failed to find another which is concealed in it, and which they had been shown before The conclusion was drawn that an unfamuliar configuration obscured a familiar, smaller one-that sensory dynamics dominates exper1ence and the effects of learning But the experimental diagram, actually, was unfamilar only as a whole. its parts, two parallelograms and a set of parallel lines forming a $\mathrm{Z}$, were certainly not unfamiliar to the experimental subjects Consequently, the conclusion is valud only if the total figure is an unanalyzable whole, which it surely is not.

In the lower diagram of figure $3, a{ }^{\prime \prime} 4^{n}$ is concealed. That it may not be recognized is evidence that sensory dynamics on occasion may override experience, in the delimitation of a figure. This is a valid point But when one looks for it, the 4 can be seen, and this is not explained by Gestalt theory A "special vector" (Kohler, 1929) is operating, but this special vector seems to be the factor of attention and expenence that is involved 
in the nonsensory figure Our problem is to find out how to modify Cestalt theory so that it can comprise this factor of attention and not have to deny the importance of learning in perception

Furthermore, Gestalt emphasis on the primitive figure, which has a marked stability, obscures the fact that in ordinary per-
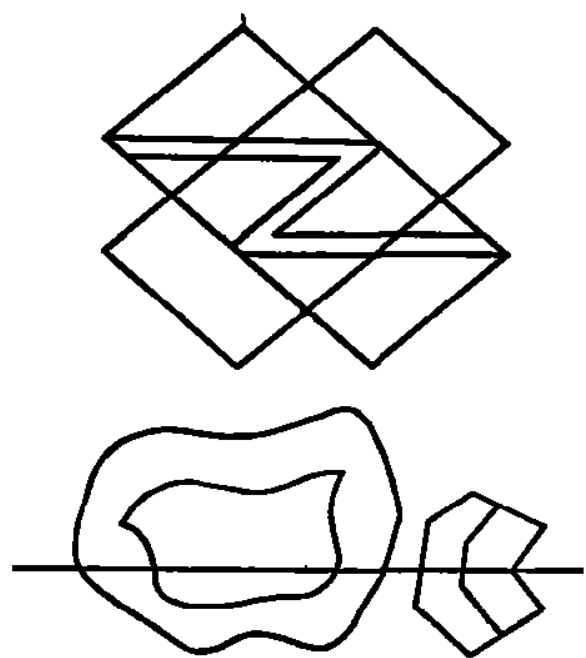

Figuris 3 Diagrams from Kohler, Gestalt psychologu, 1929 (figures 10 and 12) Courtesy of Liveright Publishing Corporation and of the author

ception any figure-ground relationship is a highly unstable one, with a practically constant fluctuation from one organuzation to another Even when one percelves the compact, clearly delimited figure which is such that any observer at once sees it, one finds that the stability of the figure-ground relation is not great, or, better, that its stability consists of a continual recurrence, instead of a constancy, of the figure. It is notonous that attention wanders, and this is another way of sayng that in perception any figure is unstable, one looks at this part of the configuration and that, and notces its corners or smooth contour, in the intervals between seeing the figure as a whole. In ordmary perception, moreover, the instability is far greater (Boring, 1933, Pullsbury, 1913) 
An adequate account of perception cannot be given in terms of a figure-ground organzation that has any great duration in tume, even when gaze is fixated at a single point in a configura. tion The fluctuations of attention which occur point directly to a fundamental importance, in any perception, of nonsensory factors. This of course has already been demonstrated for complex indetermmate figures by Carmichael, Hogan, and Walter (1932) and by Zangwill (1937), I propose that the same factors must be taken into account m the perception of square or curcle as well.

\section{"IDENTITY" IN PERCEPTION}

Identzty is defined here as referring to the properties of association inherent in a perception. The reference has two aspects first, a figure is perceived as havng identity when it is seen immediately as simular to some figures and dissimilar to othersthat is, when it falls at once into certain categories and not into others. This smularity can be summed up as spontaneous assocration, since it may occur on the first exposure to the stumulus object. Secondly, the object that is percerved as havng identity is capable of being associated readily with other objects or with some acton, whereas the one that does not have identity is recalled with great dufficulty or not at all, and is not recognized or named easily Identity of course is a matter of degree and, as I shall try to show, depends on a considerable degree of expersence, it is not unnately given.

Thorndike (1931, p 87) has presented an approach to this conception, using the term "identfiabulity," and has proposed that identifiability promotes the formation of associations If one carries the analysis a hittle further, it appears that the proposition is curcular. Identifiability is not merely a perceptible difference of one figure from another when the two are side by side, but implies a rememberable difference-1dentifiabilty is clearly, in the instances Thorndike gives, recognizability, and recogntion is one form of association Thus Thorndike's proposition is that associability affects the occurrence of associations

The real point at which he is driving seems to be that there are genume differences of associablity in different patterns. Also, more is involved in these dufferences than the number of 
trials necessary to establish recognition, there are also the spontaneous associations referred to in speaking of smilanty. Recognizability goes with selective similarity, or generalization the figure that is readily remembered is also perceived as belonging to a particular class of figures, and remembered so

An irregular mass of color or a pattern of intersecting lines drawn at random has some coherence and unity, but one such figure is not readily recognized and distinguished from others when it is seen a second time, and generalization (or simlanty) is not selective among a number of such stimul. There is not a total lack of distunctiveness and of generalization, however Two of Thorndike's figures which lack identity are not indistangurshable when seen together, and mistaking one figure for another can be called generalization Lashley and Wade (1946) dustunguush between the "so-called generalization" which means only a fallure to observe differences and the generalization which involves perception of both similanties and differences. The amorphous figure, lackung in identity, is generaluzed in the first sense only

A further lllustration of these points is found in the development of identity in the perception of chimpanzee faces by one who has seen no chimpanzees before Two anumals seen side by side are obviously different in details, but the inexpenenced observer is not able easily to remember one selectively Also, all chmpanzees at this stage look alike, the "so-called generalızation" occurs With experience the perception of identity increases Similarity is still perceived between animals, and confusion between some anumals is still possible, but there is a marked change in the perception, as shown in a much more selective simlanty of one anmal to others, and in the radical increase of the observer's capacity to assocsate even a new chimpanzee with a specific name Thus identity is a matter of degree. readiness of recognition, and the extent to which generalzzation is selective

This discussion has been meant to establish the conception of "identity" as an important property of perception which should be kept carefully distanct from the "unity" of the perceived figure (as well as from its "meaning"). Unity may be innately determined, an ummediate property of sensory dy- 
namics, whereas identity is dependent on a prolonged experience. Because these two things have not been separated in the past, it has appeared that perceptual organization is innate. Some aspects of the arganzation are present, apparently, at the first experience, but others are not.

\section{INDEPENDENCE OF UNITY AND IDENTITY IN SIMPLE FIGURES}

The examples of the preceding section dealt with complex perceptions. I wish next to review the evidence that shows that unity and identity have separate determmants even in quite simple perceptions. Seeing a circle as a single coherent object is not the same as seeng it as a dustinctive object, selectively recognizable.

Frrst, the evidence for man It bas already been said that the figure-ground segregation is good at the untral occurrence of human vision, as shown by Senden's (1932) reports, at the very time when perception of identity appears to be practically nil. Unity then can be perceived without identity Investigators (of vision followng aperation for congental cataract) aro unantmous in reporting that the perception of a square, curcle, or triangle, or of sphere or cube, is very poor. Ta see one of these as a whole object, with distinctive characteristics immediately evident, is not possible for a long period. The most intelligent and best-motuvated patuent has to seek comers painstakingly even to distinguish a trangle from a curcle. The newly seeing patient can trequently find a difference between two such figures shown together, just as the normal adult can readily detect differences between two of Thorndike's figures lackng "identufiabilsty," but the differences are not remembered. There is for weeks a practically zero capacity to learn names for such figures, even wher tactual recognition is prompt and complete

Another facet of the same lack of capacity is the farlure to generalze as normal persons do. When the patzent first gets to the point of being able to name a simple object promptly, rea ognition is completely destroyed if the object is shghtly changed or put into a new setting. The patient who had learned to name a rng showed no recognition of a slightly different ring; 


\section{Independence of Unity and Identity}

having learned to name a square, made of whto cardboard, could not name it when its color was changed to yellow by turaing the cardboard over, and so on These reports consistently indicate that the perceived whole at first vision is simultaneously unified and amorphous. There is not a sugle instance given in which the congenitally blund after operation had trouble in learning color names, but a great number in which the perception of identaty in a simple figure was poor indeed (Senden, 1932, pp. 135-141).

A second evidence comes from species differences in the perception of adentity For coherent patterns and simple groupings the figure-ground relationship appears to be the same from rat to man, so the significant differences of perceptual generalizaton (one aspect of Identity) argue strongly that figural unity and identity have separate bases. (a) The discrimination of simple geometncal figures by man and chimpanzee is unaffected by reversal of brightness relatons between figure and ground (Gellerman, 1933), a whte triangle on a black ground is generalized by these anthropoids to include a black trangle on a white ground For the rat, such a reversal completely disrupts discrimination, and no amount of traming with the white troangle alone will produce "recognition" of a black one. There is some uncertanty about the phylogenetic level at which the capacity for this generalzzation appears, see Lashley (1938b, p 144), Smith (1936), and Neet (1933), but between the rat, and chimpanzee or man, the difference in this respect seems complete (although for simple figures only, human perception of more complex figures in reversed brightness is defintely dofective, as in recogmition of photographic negatives) (b) The perception of a triangle or square by the rat is not generalized, without specific tramng, to include a similar rotated figure (Frelds, 1932) but is generalized so by chimpanzee and by twoyear-old children, although a corresponding head rotation also occurs (Gellerman, 1933). (c) Perception of a triangle is not generaluzed etther by rat or chmpanzee to include a triangle made up of small circles, but the generalization is made by twoyear-old chldren (Gellerman, 1933, Lashley, 1938b).

Thus the perception of identity is different in different mammals, the perception of primitive unity is practically the same. 
Further endence of the independence of unity and identrty is found in the peculiar "equivalences" or generalizations often observed in rat behavior, and in the anomalies to be found in the relative difficulty with which the rat learns to discriminate between certain patterns Two discrimunations that are equally easy, from the human point of view, present very unequal diffculty for the rat For normal man a square and a circle are no less obviously distinct than erect and inverted triangles, yet the rat readily learns to discriminate between the triangles and consistently has trouble with circle versus square (Lashley, 1938b, pp 155, 156) Some anumals that have learned other patterns (thus showing that their acuity is sufficient for the task) show no sign of discriminating crrcle and square at all A five-pointed star and an $H$ are for man clearly, inevitably distnet, whle the rat distnguishes them whth difficulty and no faster learning occurs than with quite irregular figures (Lashley, 1938b, p. 157). Such facts mean a great difference of rat from man in percelving the identity of simple, regular figures.

The same is evident in the patterns which may be "equivalent" for individual rats, and in some failures of equivalence One anmal may transfer his response from an erect trangle (versus an inverted one) to a single horizontal line low on the stimulus card (versus another higher on the card), while another animal fals to discriminate the complete trangles with which he was trained, when a slight change is made of their positions on the respective stimulus cards. It is very doubtful in such cases that the rat has perceived the pattern as a distinctive whole, and it seems that a response is frequently determmed by only a part of a figure as simple as a triangle

Lashley (1938b, p 182) has recognized this, but interpreted it by comparing the rat to a sk-jumper who does not pay attenton wn his jump to nonessentrals such as the shape of a spectator's hat The general interpretation was made that there is little significant difference in the perception of simple figures by rat and man, but this conclusion is not supported by the evidence

When identity is distinguished from unity, we find great species differences in the perception of identity and not in what is seen as a primitive unity. The analogy between the rat's per- 
ceiving all the detall of a trangle in the jumping apparatus, and the ski-jumper's perception of a hat, is valid-except that it sets the human subject a much more difficult task It is mconceivable that a ski-jumper would not perceive a large triangle as such, fully structured, if it lay close to and marked the point of safe landing in an otherwise plain field, as it does for the rat in the jumping apparatus The rat, however, may respond selectrvely to only the part of the figure whrch lies nearest the point to which he jumps, although his field of vision is wide and although (as Lashley has demonstrated) he perceives much more than he has to in making a discrimination

These considerations are the more convincing because the evidence of the rat's frequent falure to see the triangle as a distunctive whole figure comcides with Senden's description of the congenitally blind after acquinng vision-the normally reared rat, and a man seeing for the first time, both have precisely the same kind of difficulty

It is reasonable to conclude that the perception of identity (as defined) follows the same prnnciples in rat and man but is much more highly developed in normal man Since there is no evidence of any clear difference of the primitive figure-ground organization perceived by these two species, but strong suggestions that it is the same for both, the evidence is consistent with the idea that identity and figural unity have separate physiological bases They are, that is, psychologically independent This makes it possible to suppose that additive processes may occur in the development of identity without denying that the figure has a primitive unity

\section{LEARNING TO PERCEIVE SIMPLE FIGURES}

The facts already adduced in the last section have indicated a role of learning in the perception of triangle or square as distinctive wholes The idea that one has to learn to see a triangle must sound extremely improbable, and so I shall now present the evidence to this effect more systematically

We have seen that the perceptions of the congenitally blind after operation are almost completely lacking in identity Senden (1932, pp 155-157) reports cases in which there was an 
ceiving all the detall of a trangle in the jumping apparatus, and the ski-jumper's perception of a hat, is valid-except that it sets the human subject a much more difficult task It is mconceivable that a ski-jumper would not perceive a large triangle as such, fully structured, if it lay close to and marked the point of safe landing in an otherwise plain field, as it does for the rat in the jumping apparatus The rat, however, may respond selectrvely to only the part of the figure whrch lies nearest the point to which he jumps, although his field of vision is wide and although (as Lashley has demonstrated) he perceives much more than he has to in making a discrimination

These considerations are the more convincing because the evidence of the rat's frequent falure to see the triangle as a distunctive whole figure comcides with Senden's description of the congenitally blind after acquinng vision-the normally reared rat, and a man seeing for the first time, both have precisely the same kind of difficulty

It is reasonable to conclude that the perception of identity (as defined) follows the same prnnciples in rat and man but is much more highly developed in normal man Since there is no evidence of any clear difference of the primitive figure-ground organization perceived by these two species, but strong suggestions that it is the same for both, the evidence is consistent with the idea that identity and figural unity have separate physiological bases They are, that is, psychologically independent This makes it possible to suppose that additive processes may occur in the development of identity without denying that the figure has a primitive unity

\section{LEARNING TO PERCEIVE SIMPLE FIGURES}

The facts already adduced in the last section have indicated a role of learning in the perception of triangle or square as distinctive wholes The idea that one has to learn to see a triangle must sound extremely improbable, and so I shall now present the evidence to this effect more systematically

We have seen that the perceptions of the congenitally blind after operation are almost completely lacking in identity Senden (1932, pp 155-157) reports cases in which there was an 
immediate perception of differences in two figures seen together, but also a number of instances in which even this was not possible Thus a patient sometimes saw differences between a sphere and cube, sometimes not (p. 91) Color has been found to dominate form persistently in the first vision of these patients Eleven months after operation the color names learned by a patient in hospital were retained, but the little that had been learned of form was forgotten (p 135). An egg, potato, and cube of sugar were seen by a patient repeatedly, unth naming was prompt, but then were not recognized when put into colored light, the cube of sugar was well named when it was seen on the table or in the investigator's hand but not recognuzed when suspended by a thread with a change of background (p 138).

Such patients, when learning has proceeded far enough, manlfest the characteristic generalizations of the normal person, so the initial difficulties are not to be put down to structural defects of the sensory apparatus (Senden, pp 173-175).

Ruesen (1947) has fully confirmed the conclusion that ordinary visual perception in higher mammals presupposes a long learning period. His observations concerning the almost complete visual incapacity of chimpanzees reared in darkness, and the slowness of learning, are of the greatest importance. They show that Senden's simular results with man are not due to some madequacy of the clinical tests, nor peculiarly human.

The course of perceptual learning in man is gradual, proceeding from a dominance of color, through a period of separate attention to each part of a figure, to a gradually arrived at identification of the whole as a whole an apparently simultaneous instead of a serzal apprehension $A$ patient was tramed to discriminate square from triangle over a period of 13 days, and had learned so little in this tume "that he could not report their form without counting corners one after another - A And yet it seems that the recognition process was beginning already to be automatic, so that some day the judgment 'square' would be given with simple vision, which would then easily lead to the belief that form was always sumultaneously given" (Senden, 1932, p. 160) The shortest time in which a patient approximated to normal perception, even when learning was confined 
to a small number of objects, seems to have been about a month

It is possible then that the normal human infant goes through the same process, and that we are able to see a square as such in a single glance only as the result of complex learning The notion seems unhkely, because of the utter simplicity of such a perception to the normal adult But no such argument can be vald, since Lashley (1937) has shown that subjective simplicity and immediacy may be very deceptive as an index of physiological simplicity. There are moreover residual traces of learning in normal perception, and hints of its complexaty

Gellerman (1933) reports that chimpanzees and two-year-old children recognized a triangle that had been rotated through $120^{\circ}$ from the training position, but (m the one protocol that is given) responded selectively only after a head rotation, and persistent head rotation continued in the later discriminations. Older human subjects do not need to make the same receptor adjustment to recognize the figure in two positions, and so this generalization may be a learned capacity, simple as it seems to us.

Consider also the following evidence, which is suggestive though perhaps not conclusive. When a simple figure such as square, curcle, or triangle, subtending a retinal angle of from $2^{\circ}$ to $10^{\circ}$, is fixated at one point, it tends in a second or so to become almost amorphous except near the point of fixation. The effect is not due to fatigue alone, nor to poor acuity outside the macular zone' sunce (1) a single straight line does not suffer nearly as much, (2) shiftung fixation back and forth between two corners of a triangle does not restore the clanty with which the third is seen, and (3) an $8^{\circ}$ curcle drawn with a line $2 \mathrm{~mm}$. wide, and a $4^{\circ}$ crrcle drawn with a line $1 \mathrm{~mm}$. wide, seem to give approximately the same effect

The factors involved are evidently complicated, it will be found, for example, that with a large figure merely imagining eyemovements (of following the contours) will restore definition of the figure. Also, these "umaginary" eyemovernents, or subliminal actuvations of the motor system, occur more frequently and are less easy to control in lookng at a smaller than at a larger figure, and it is hard to be sure that the szze of the figure is un- 
important. But this at least seems definite, that a stable, clear, and effective perception of crrcle or square is more possible with eyemovement than without Once the question is asked, anyone can verify for himself the falsity of the implicit assumption (usually made in the study of perception) that the figure acts always as one, without a reinforcing perception of its parts as distinct from the whole.

My point is not that eyemovements are essential to perception by a sophisticated observer (nor, in the following paragraph, that they are completely necessary for an image), but that the perception is definitely clearer, more effective, with them than without. This is really an evident fact It is to be interpreted in the light of all evidence, cited above, showng that the perception of square or circle is slowly learned and depends orighnally on multiple visual fixations

Directly in line with such phenomenological observations are the following introspections I find it very difficult to have a clear image of a triangle, square, or circle without imagining or actually making a senes of eyemovements. Several others, asked to make observation on this point, have reported the same thing It is hard or impossible, that is, to have a clear image of a triangle as fixated at one point Eyemovements defintely improve the "image" They do not take the form, necessanly, of following the figure's contours, but are apt to jump from point to point, perhnps three to four points in all Thus the distinctiveness of the image is not merely in the eyemovement pattern, for approximately the same series of eyemovements may contribute to a good image either of circle or square. Activation of the motor system, overt or implicit (even possibly within the cerebrum alone, with no activity of the final common paths), contributes essentially to the development of visual integration without being sufficient to it As I have said, such evidence is hard to evaluate, but it points to the same conclusion as Senden's evidence, already crted, and is supported by it.

Clark and Lashley (1947) have confirmed the observations of Kennard and Ectors (1938) and Kennard (1939), and have provided what appears to be an independent confirmation of the argument above Kennard found a one-sided loss of vision by monkeys on extirpation of the opposite frontal eyefield, a cortical 
motor area for head-and-eye movement. Clark and Lashley have demonstrated this phenomenon convincingly, with an adequate method of testing The most significant and strikng observation was startle by the monkey when an object was passed from the blind side into the seeing side, at the moment of passing the midline. One might have argued that the animal could "see" an object in his apparently hemianopic field but was not able to move his eyes toward it. The observation referred to rules that interpretation out, and other observations showed that the hemianopia is a genuine fallure to see (though it is transient, disappearing in a week or two).

Now the question is what the motor cortex can have to do with visual perception-unless perception intumately involves a motor activity, liminal or sublimunal There is no reason to think that the frontal cortex has anything to do with the receptron of visual sensation, and the alternative seems to be that it must have something to do with the elaboration of sensation into visual perceptions. In Chapter 5 will be found a treatment of perception which supposes that perception of even a sumple object involves a "phase sequence." This is a cham of central cortical events with motor links Although the motor activations may be subliminal and do not always produce overt response, their role is essential in any perception This would account for the observations of Kennard and Ectors, and Clark and Lashley, which therefore can be considered to be a confirmation of the argument of Chapter 5 as well as the argument of the preceding paragraphs

\section{CONCLUSIONSS}

Animal experuments and the human clinical data alike indcate that the perception of sumple diagrams as distnctive wholes is not immediately given but slowly acquired through learning. Introspective observations which would not carry much weight in themselves appear to agree fully with other evidence, showing vestiges of a summative process involved in perceiving the identity of circle or triangle, although such a figure is seen by the adult clearly and is effectively discriminated at a single glance, there are still traces left of complexities such as the 
learning process described by Senden would produce, which for normal persons must have occurred in early infancy and which makes the unified perception possible.

The subjective experience of an irreducible simplicity in the perceptron of square or curcle may then be fully analogous to the illusion of the mage of the letter-square (Woodworth, 1938, p. 42), where the subject thinks he has an actual image of the square but can be shown not to have." Although the percepton of identity is good at a glance, it is umproved by several glances at different parts of the figure This process of "successive part reinforcement," as an ald to perception, exists at the same time as an essential unity of the whole, and theory must provide for the additive process, with its motor elements, as well as for the primitive unity.

This argument is not in any way a return to the old idea that sensory integration occurs solely through motor activity, or that the distunctuveness of a perception is solely or mainly due to distinctive eyemovement. We know that this is not true. But there are three theoretical possibilities, not two (2) perceptual integration is wholly the result of motor activity, (2) it is wholly independent of motor activity, and (3) the motor activity is important but not all-important-the position that is taken here

Grant fully that visual integration cannot be reduced to a syathesis of unrelated elements through effector activity, and the question remains, how much suguificance the motor factor

- The experiment is as follows The subjoct is shown a diagram such as

$$
\begin{array}{rrrr}
x & e & a & \mathbf{a} \\
\mathbf{r} & 1 & 1 & \mathbf{s} \\
\boldsymbol{o} & \boldsymbol{E} & \mathbf{z} & \mathbf{g} \\
\mathrm{d} & \mathbf{y} & \mathbf{u} & \mathbf{p}
\end{array}
$$

and studies it intl he has, apparently, an image of the whole square and can "look at" it and read the letters of, one by one if he really has such en image, it will not matter m what durection he is asked to "read." Actually, it is found that the subject cansot reproduce the letters as fast from tight to left as from left to nght, or promptly give the four letters, $p_{1} z, z_{1}, x_{\text {, }}$ that make up the diagonal from lower nght to upper left. So what seems a simple, immedrately given image of the whole is actually a senal reconstruction of parts of the figure An "image" of trangle or square is simpler, longer practlced, but may be fundamentally the same. The perception of such figures, also, may involve a temporal sequence 
may still have Receptor adjustment (head-and-eye movement) is the most prominent feature of visual perception whether in rat, chumpanzee, or man-except in long-practiced habits. The assumption has been tacitly made that the adjustments are unimportant for theory (once it was shown that they were not the whole answer). The fact of eyemovement has been treated only as a further (negative) evidence that the locus of excitation in the retina is unmportant, since the changing retunal projection does not disturb perceptron. But obviously another point of vew is possible. The thesis of this chapter is that eyemovements un perception are not adventitous They contribute, constantly and essentially, to perceptual integration, even though they are not the whole ongin of it. 


\section{Field Theory and Equipotentiality}

The last chapter examined the proposition that perception is a unitary process, in all its aspects We can turn now to an mvestigation of the two theories, Kohler's and Lashley's, that have resulted from that idea In effect, this will be a critique of the theory of perception, since Kohler and Lashley are the only writers who both recognize the real problem of the neural mechansms of perceptual mtegration and attempt an adequate solution. Others have avoided the crucral difficulty of specifying the way in which perceptual generalization occurs Either like Pavlov $(1928,1932)$ they have not seen the difficulty at all, or like Hull (1943) have put it to one side for solution later. Hull has discussed the problem, but only to show that it as not necessarily insoluble for his theory of learning (Hull, 1945). For the present, Kohler and Lashley are the only ones who have attempted to say where and how perceptual generalization takes place

For reasons already mentioned, however, the line of thought that they have chosen may be a blind alley, and it must be shown that there are other possible solutions. Until someone challenges the theory of innate stimulus equivalence and equipotentiality, and of neural fields and gradients as the only mechanism of integration, any theory with neural connections in its ancestry is damned before it is born. Being gravd, and so disapproving of infant damnation, let me look for another gospel. 


\section{CLARIFICATION OF TERMS AND ISSUES}

As elsewhere, the issues here have sometumes been lost sight of $\mathrm{n}$ the dust of a prolonged battle There is one main question whether recognition, or a selective discriminatory response, requires the excitation of specific neural cells or not

The doctrine that it does not, we can refer to as equipotentrality. Kohler does not use the term, but his position is the same as Lashley's on this point, and a single term will do

Explanations of the supposed equipotentiality differ greatly in some respects but agree in their stress on patterning in the central nervous system as against locus of excitation so we can again use a single term, configuration theory, to designate Kohler's (1940, Kohler and Wallach, 1944) explanation by electrical fields in the cortex and Lashley's (1942a) alternative idea of urradiating waves of excitation and therr interference patterns.

Next, sensory equipotentiality can be coined for Lashley's "equivalence of stimuli," which is ambiguous One may want to refer to a limited degree of equipotentality, in afferent structures, without accepting the whole theory of equpotentiality in central action, and this term can be used for the purpose

"Equivalence of stimul" has a double reference It may mean only (1) that different stmulh can arouse the same response. This is an observed fact of behavior, whatever one's interpretaton of the fact But Lashley has also used the term to mean (2) that it does not matter what sensory cells are excited in order to get a certain response, and this is interpretation The fact that two different patterns have the same effect may be due to eyemovement, or to multuple learning processes (the position adopted here), or it may be because similar patterns of excitation have the same ultimate motor effect, innately and necessarily, whether the receptors excited are the same or not. Let us then separate fact from interpretation, and let us refer (1) to the behavioral evidence in speaking of the equivalence of stzmull, and (2) to a partcular interpretation in speaking of sensory equipotentiality.

Finally, the idea of equipotentiality itself should be made more explucit. "Potentuglity" literally implies only that different 
cells are able to acquire the same function in behavior. Anyone must agree that this can happen, with separate learning processes It is the basic idea of conditioning But Lashley has given the word another meaning, that all cells in a system necessarly do acquire the same function, when excited in a given pattern, so that separate learning processes are not necessary for habits unvolving dufferent stumuli or different parts of a cortical field.

Suppose for example that a habit is set up in thes way. the stimulus is a curcle, let us say, that falls always on the same retinal cells in the lower right quadrant, the response is a movement of the left hand The stimulation then 13 confined to one half of the visual area in the nght hemisphere, and the response is determmed by motor systems that are also in the right hernisphere. Equipotentrality implies (1) that any other retunal cells, excited in a crrcular pattern, will elicit the same response-with ether left or nght band, (2) that the right hemisphere may be exturpated, and the left will be found then to have "learned" whatever the night did, and (3) that this transfer of learnug from one set of cells, primanly excrted, to other sets does not depend on an earlier experience that set up connections between them The idea is that the transfer is an innate property of the central nervous system, so that it is not possible for a particular habit to depend on any particular neural cell within an anatomcal system, in any circumstances. If a whole system is exturpated, habits will be lost (as when both visual areas are removed), but it is not possible for a habit to be dependent on one half of an anatomical system only (for the habit to depend on one visual area alone, or on any part of $2 t$ )

This is obviously a rather extreme position An alternative hypothesis to be proposed later recognizes a hmited field action in the sensory projection areas, and something like a limited equipotentiality between cells that are in functional parallel in physiological systems (this will be clear later on), but in the main it derives transfer of response from earher-established habits and specific connections.

The issue is thus defined. Wo must recogneze that Kohler and Iashley have been completely successful in their original $p$ attack on stimulus-response theory such as Watson's. No one 
now believes that retinal cells acqure durect connections with muscle cells, as in a child's learning to say "cat" when he sees a cat. The elimmation of such oversinplified forms of switchboard theory was a fundamental contribution. One need only glance at the Journal of Nenrophystology to see that there are gradients of neural activity, there is some mutual influence of nerghboring parts as an excitation is conducted from the retuna to area 17. Electrophysiology has fully confirmed the value of such rdeas, and they must enter into psychological theory.

But in fact, they have been generally accepted already, so this is no longer an issue Pavlov's uradiation and concentration is a feld action Hull and Spence both make use of the notion of gradients. The question now is whether gradients and fields are the only mechanism of a selective neural action or whether they are combined with an equally important mechanusm of connections and speczelzzed conduction paths

In short, configuration theory represents two things It has, been, first, a necessary corrective to earher ideas, such as Titchener's and Watson's. This positive contribution must be recognuzed as an important step forward. But there is also an over-reaction from punctate sensory elements and sensonmotor connections that is about as extreme as these older ideas. As a theory, therefore, configurationusm is just as vulnerable as. structuralsm and the early behaviorism were.

Now let us see what the evidence is by whrch we can accept or reject field theory and equipotentsality. Some of the evidence that has been used to support these ideas actually is not relevant to the question, and such evidence can be discussed first, leaving the more relevant evidence for later consideration

\section{THE PERCEPTION OF BRIGHTNESS, SIZE, PITCH}

In the literature, the perception of relative intensity has been used almost entirely by writers on configuration theory for the embarrassment of learning theory Actually, it is no easier for configuration theory to explam, and so is not really relevant to the debate between the two. It has been thought to be crucial, however, and so must be considered here Man or anmal tends to perceive relative rather than absolute intensity, extent, or fre- 
quency. One can readily tram an anmal to choose the larger of two surfaces; it is extraordmarily hard to get hm to choose a particular size, except when differences are very great These facts are psychologically important, and they provided a deczsive answer to learning theory in its older forms, but we know too little of the physiology of the relative perception to use it as evidence for or against any currently mamtamed psychological theory.

The actual expermental evidence is as follows, taking the discrumunation of brightness as an example.

A rat is trained to go toward a light of intensity 1 and to keep away from intensity 2 ( $e$, twice as strong as intensity 1) $\mathrm{He}$ is then presented with intensities 2 and 4 , and chooses (if he chooses at all) intensity 2-which he was taught to avoid (In the test, discrimunation may break down, choices being made at random This happens perhaps less than half the tume, but a consistent choice of an absolute stimulus value never occurs in the rat in these circumstances, and the relative value is what the anumal discriminates, when he does discrimmate.)

Or, if the rat is taught in the first place to avoid an intensity 1 and to choose intensity 2, in the test with 2 and 4 he will reject the stumulus be was tramed to respond to in favor of a new one, intensity 4 . This happens even when the anumal has been reared in total darkness and has never in his life seen that stumulus before This is, so far as one can see, still a conclusive answer to any theory of behavior that deals in connections from specific rods and cones to specific muscle fibers (Hebb, 1937b).*-

But the perception of brightmess is commonly cited in a broader context, as supporting configuration theory in general against "learning" theory in general. By implication, it is treated as support for the idea that discrumination is not dependent on the excitation of any particular cells, at any neural level. This does not follow. The truth is that no one has produced any good explanation of the relative perception, so it does not support one camp agaunst the other, and the truth is, also, that

- This author, however, seems to have supposed that such experiments rule out all possibulity that connections are the basis of learning The hypothesis presented in the following section is enough to show that the conclusion was unwarranted 
we know little or nothing about the locus of the cells whose actrinty mediates the perception "brighter," or "smaller," or "higher" (m pitch), so the facts neither deny nor support the hypothesis of equipotentrality.

We know, with reasonable certainty, that the training process does not form a connection all the way from receptors to effectors, but it is still possible to suppose that connections may be formed at some intermedate levels. I shall outline here a possible hypothess, manly to show that we must know more of the physiology of sensation before bnghtness perception (for example) can be made an argument for or agamst any existent theory.

\section{AN HYPOTHESIS OF BRIGHTNESS PERCEPTION}

Marshall and Talbot's (1942) treatment of visual acuity makes it possible to assume that the perception "Brighter" depends on the activity of particular cortical cells, but not of particular receptor cells We take for granted that intensity 2 excites a larger population of first-order (retinal) neurons than intensity 1 and with a higher rate of finng Intensity 4 excites a still larger population The fact that 2 and 4 may elicit the same selective response is an argument for a sensory equipotentality, but the equipotentiality may disappear farther on in the system.

Marshall and Talbot point out that the whole vsual system, from receptors to the several layers of the cerebral cortex, must act to damp strong stumulations, amplify weak ones See what this means for the behavioral problem At each synaphe stage in transmission the damping, or amplification, increases A level $X$ may be reached in the system where the degree of activity is constant for an adapted retnal area, with a brightness in the middle range The amount of activity at $X$, then, will be the same whether the eye is adapted to intensity of 1,2 , or 4 .

This of course is true only in adaptation The system must remain very sensitive to changes of stumulus intensity Therefore, when the eye after being adapted to 1 is focused on 2 , the level of acturity at $X$ goes up, say, from $n$ impulses per second to $1.5 \mathrm{n}$. The same would happen when the eye adapted to 2 
is focused on 4 In one part of the system, the same central neurons might thus be active in the perception "brighter," regardless of absolute stimulus values (In other parts, the cells that are aroused would stll correspond partly to the absolute untensxty, and this helps to account for the fact that absolute values can be detected, though the tendency is toward the relative discrumination.)

My point is not that thus has explained the relative perception. I do not suppose that the problem is as simple as this has made it sound. The point is that the known facts are not unconsistent with the idea that the perception "brighter" involves an actuity in specufic neural cells at some point in the system, and thus that a perception of relative values is not incompatible with some specific neural connections Sornething else, a "sensory equipotentrality" akm to neural gradients and field action, is certainly involved; but the establishment of new symaptic connections is not ruled out as the basis of an animal's learning to respond to the brighter of two surfaces.

In the next two chapters some neurophysiological ideas are proposed about visual perception They could be elaborated speculatively to deal with the perceptuon of brightness and stze, though I have not done so. The perception of size, brightness, and pitch should be written down for the present as not yet accounted for, by any theory. Before they are, we shall have to know much more about the physiology of sensation.

\section{THE PERCEPTION OF PATTERNS}

In the perception of patterns we reach surer ground, having more of the relevant physiological unformation available and beng better able to see what bearng the facts have on the theory of equipotentiality.

An anmal that has learned to look for food behind a small square and not behind a small circle will, withoust further training, choose a large square and reject a large circle $5 \mathrm{mall}$ and large squares are "equivalent strmuli." If each is perceived with a single fixation, it is impossible for the total pattern in each case to excite the same set of retinal cells. The current treatment of perception disregards eyemovement, the unference has 
been drawn, therefore, that the locus of the cells stimulated by the square is unimportant Only the pattern of stumulation matters

But we have seen, in the last chapter, that eyemovement cannot be disregarded A rat traned to jump toward a square does not respond only to the total figure, but at times clearly responds to parts of it, as separate entities Eyemovement has never been controlled in animal studies of perception. If the rat fixates parts of the figure in succession the result is to superimpose its parts, one after another, on the same retunal cells that were involved in the onginal traming The locus of excitation may then be essentral. Evidently the equivalence of similar patterns dufferent in stze does not in itself provide support for the theory of equipotentrality

For the endence to become crucial, two conditions must be fulfilled. The equivalent stumuli must be shown to be conducted to different parts of the central nervous systern, and there must have been no previous expernence that could have set up connections between those parts before the experiment began

One of these conditions has been met by Levine's (1945a, 1945b) experment with pigeons The optic chrasm in birds $2 s$ completely crossed, so one eye conducts to one optic lobe, the other to the other. Levine achieved the truly surpnising result of finding transfer for patterns in the lower half of the visual field and not in the upper half For configuration theory, the best that can be sard with these results is that there is a limited equipotentrality, between parts of the optuc lobes (from the opposite point of view, these results definitely refute the theory of a general equpotentzality in vision).

Levme's experinent did not, however, control earher expernence in his burds, and it remains possible that even the limited equpotentiality suggested by his results is illusory It is possible that patterns in the lower half of the bird's visual fieldas when the bird is foraging on the ground, or alighting on a perch that extends into the field of vision of both eyes-had established, by perceptual learning before the experiment began, interconnections between the two optic lobes which might later be a basis of transfer This would not require that the bird 
have learned beforehand the actual patterns used in the exper1ment, only that the parts of those patterns (or "perceptual eler ments" see Chapter 5) have been learned. That this possibulity is not far-fetched will be seen as we consider next the instantaneous recognition of patterns by man, where it is evident that the perception of one or two parts of a figure may be the clue to recognizing the whole.

Human beings readily recognize certain patterns in the tachistoscope The time of exposure of the pattern is of the order of one-ifth of a second The subject cannot fixate the separate parts of the pattern one after another, and cannot adjust his eyes in advance so as to make it fall on any particular retinal cells This might be interpreted as equpotentiality, but there are facts that make that interpretation practically impossible.

I propose that the human capacity for recognizing patterns without eyemovement is possible only as the result of an intensive and prolonged vsual training that goes on from the moment of birth, during every moment that the eyes are open, with as mcrease in skall evident over a period of 12 to 16 years at least The evidence supporting this proposition is found (1) in the errors made in tachistoscopic recognition, (2) in the way in which reading skill develops, and (3) in the rate and course of learning by the congenitally blind after operation.

1. The recognition of patterns momentarily perceived, as in the tachistoscope, is extremely defective except with simple and conventional patterns, or very simple combinations of them. The process is exactly that named by Woodworth (1938) "schema with correction" This means that the pattern is perceived, first, as a famular one, and then with something missing or somethung added The something, also, is famuluar; so the total perception is a melange of the habitual.

- In particular, the slope of lines as they extend into the fields of both eyes sumultaneously This explanation by a learning process is not, however, the only alternative to equupotentality Another possibility is that part of each optic lobe, receiving impulses from the uppes hemretna only, has a point-to-point projection to the other lobe Transfer would thus be mnately provided for The explanation by learning, however, should be examined flrst, by repeating Levine's experiment with pigeons reared in darkness and kept in darkness except when they are ectually being tranned or tested 


\section{The Perception of Patterns}

The subject's reports are such as "a triangle with the top cut off" or "a square with a crooked bottom" It is thus clear that the subject is not only responding to the diagram as a whole, he perceives its parts as separate entities, even though presentation is so brief Errors are prominent, and such as to show that all the subject really perceives-and then only with rough accuracy-1s the slope of a few lines and ther durection and distance from one another. A drawing or a report of what is seen tachistoscopically is not unluke a paleontologist's reconstruction of early man from a tooth and a rib There is a clear effect of earlier experience, filling in gaps in the actual perception, so that the end result is ether something familar or a combination of famllar things-a reconstruction on the basis of experience.

All that is needed to account for the recognition of pattern without special adjustment of the eyes, to the extent that such recognition really occurs, is ability to recognize roughly (1) the slope of line, (2) degree of separation of points, in any retinal projection. These in combination cover intersection of lines, sharp inflections of line (ar corners), and curvature of line (by the variation of slope at different points, and the absence of sharp inflection) What I am proposing, then, is that, during the contnuous, intensive, and prolonged visual training of infancy and childhood, we leam to recognize the direction of line and the distance between points, separately for each grossly separate part of the visual field.

- Here, as elsewhere, I have chosen to be more definite than is actually required by the theory to be developed, this is done whth the conviction (1) that vagueness is not desirable as such, in psychological theory, and (2) that the more definite assumption may turn out to be correct, and at any rate should be erplored.

If it as not correct, however, the later theorkang is unaffected An alternative possibility to be considered is that some form of Lashley's interference-pattem theory may hold in modified form Lashley has used the theory to explain perception of a square or trangle as a singlo unified process, and it will be seen in the later part of ths chapter that the theory In this form cannot be malntaned, but it might be feasible to apply it to single lines unstead Then the stimulation from a line of a certain slope in one part of the visual field would be mnately equivalent to the stimulation of a sumilar line in another part of the field I know of no evidence that opposes this, but no endence for it exther.

Psychologically, auch an explanation might be more plausible, what it 
I have already admitted that such ideas sound unlikely The perception of simple objects seems so simple and drect, so obviously ummedsate, that it cannot be complex and the result of a long-drawn-out learning process. But it was shown in Chapter 2 that the learning process is necessary, that the end result is compler, whatever it may seem

It should be noted that these ideas do not mean that each separate row of suggle cells in the retina must be separately conditioned for the perception of horizontal line $(e \mathrm{~g})$ in different retmal projections. Marshall and Talbot's discussion of visual acuity implies a signeficant overlap of excitation, at the level of the primary visual cortex, from lines that have distunct retunal projections. It is not clear how great this spread is, in terms of retinal angle, but it may be of the arder of $2^{\circ}$ in central vision and considerably greater at the periphery Accordingly, the number of separate visual habits necessary to ensure the recogmition of horizontal line in any retmal projecton might be, perhaps, not greater than 10 or 15 . Lines of slightly different slope, likewnse, would not have to be learned separately (remember that accuracy in discriminating slope tachistoscopically is not great) The number of separate perceptual habits needed to account for tachistoscopic vision, consequently, is by no means infinte

Now consider how intensive and prolonged the human visual training is As we shall see later, every single movement of the eyes, when exposed (as they always are) to an unevenly lighted field, produces a number of excitations corresponding exactly to the excitation from a number of lines all parallel to the durection of eyemovement. The eyes are constantly movng when the subject is awake. Every waking moment then provides the necessary conditions for reinforcung the perception of lunes, in every part of the retina.

would postulate is that parallel "ndges" of activity in area 17 would tend to excite the same, duffusely arranged, cells in $18 \mathrm{My}$ neural schematizing, in the following two chapters, would then $g 0$ on as before, but without the necessity of supposing that different parts of the retina must be separately conditioned to produce recognition of the slope of line in any part of the field

The question is, I believe, open to expenzmental test, and will be investugated 
We have already seen that it takes months for the first direct apprehension of a figure such as a plain, well-marked triangle to be established The normal human infant, apparently, reaches this stage quite early in life, but his further training contenues every moment that his eyes are open, and must extend his capacity for prompt recognition of patterns fallug outside the macula.

2. Speed of reading increases up to the age of 12 to 16 years, or perhaps even later This speed, depending on the instantaneous recognition of larger and larger blocks of letters, may be regarded as a function of the training of peripheral vision, it umplies that learning to recognize a word ummediately (as a single Gestalt) in direct central vision does not mean recognizing it ummediately at any angle of regard, though this is what the theory of equipotentiality requires.

It might be argued that the theory does not require this, because of differences of acuity between central and peripheral vision Peripheral recognition of a word may depend, for example, more on its general outline and less on particular letters. It might be argued, that $2 s$, that an increased speed of reading depends on learning to use different cues from those used in central vision. This may well be true, but such an argument abandons the fundamental thesis of equipotentrality, namely, that the same propertues of form are perceved whatever the retmal projection, provided acuity is sufficient Above all, such an argument would umply that peripheral recognition and central recognition depend on separate visual habits, which is the argument of this chapter.

More direct evidence on the point is avaulable, however, than when the above considerations were first formulated These considerations suggested the need of a re-examination of some features of tachistoscopic perception, and this has been begun by $M$ Mishkin and $D$ Forgays Their experments show drectly that reading does not tram all parts of the retma in the same way, even when acuity does not enter the picture. The work will be reported in detal elsewhere, in summary, the following results have been obtamed. Fluent readers of English, used to reading from left to night, are able to recognize words to the right of the fixation point between two and three times 
as well as words to the left of fixation. Readers of Jewish (in which the words run from nght to left) recognize more words to the left of fixation These results can be obtained when the subject does not know whether an Englush or a Jewsh word is coming next, or whether it will fall to the night or the left of fixation-that is, with a random order of left-right, English-Jewish presentations The subjects that have been studied were not very fluent in Jewssh, apparently, and the left-right dufference, with Jewish words, was not statistically significant $(P=015)$; but it is certan that whatever factors make the English word better recognized to the right of fixation does not affect recognition of Jewish words. Either there is no difference, or the Jewish word is recognized better to the left

This shows directly that there is not an equipotentiality between the left and the nght hemiretina, or left and nght occipital cortex, in man. A learned response can be more readily elicited from one part of the retina than another, which provides a good deal of support for the assumption, made in these chapters, that a separate learning process is necessary for the elicitation of the same response by stimulation of a separate region in a receptor surface

3. Finally, there are Senden's explicit reports of the way pattern vision develops after the congenitally blund are operated on (see Chapter 2). The patient, despite weeks of practice, must still count corners to distunguish a square from a trangle here there is no unimportance of the locus of stimulation, no endence of equipotentiality When the patient first gets to the point of naming an object promptly, the recognition is destroyed by puttung the object into a new settung or by changing its color. The significant fact is that the characterstic normal generalization shows up later, after a prolonged and arduous traming process. The evidence is clearly that the apparent equipotentiality is the sequel and product of learning, the perception of pattern is specific and limited at first, generalized only with further practice; and in these cases there is no support whatever for the idea that when a pattern is recognized at one angle of regard it will be at once recognized in any other. 
as well as words to the left of fixation. Readers of Jewish (in which the words run from nght to left) recognize more words to the left of fixation These results can be obtained when the subject does not know whether an Englush or a Jewsh word is coming next, or whether it will fall to the night or the left of fixation-that is, with a random order of left-right, English-Jewish presentations The subjects that have been studied were not very fluent in Jewssh, apparently, and the left-right dufference, with Jewish words, was not statistically significant $(P=015)$; but it is certan that whatever factors make the English word better recognized to the right of fixation does not affect recognition of Jewish words. Either there is no difference, or the Jewish word is recognized better to the left

This shows directly that there is not an equipotentiality between the left and the nght hemiretina, or left and nght occipital cortex, in man. A learned response can be more readily elicited from one part of the retina than another, which provides a good deal of support for the assumption, made in these chapters, that a separate learning process is necessary for the elicitation of the same response by stimulation of a separate region in a receptor surface

3. Finally, there are Senden's explicit reports of the way pattern vision develops after the congenitally blund are operated on (see Chapter 2). The patient, despite weeks of practice, must still count corners to distunguish a square from a trangle here there is no unimportance of the locus of stimulation, no endence of equipotentiality When the patient first gets to the point of naming an object promptly, the recognition is destroyed by puttung the object into a new settung or by changing its color. The significant fact is that the characterstic normal generalization shows up later, after a prolonged and arduous traming process. The evidence is clearly that the apparent equipotentiality is the sequel and product of learning, the perception of pattern is specific and limited at first, generalized only with further practice; and in these cases there is no support whatever for the idea that when a pattern is recognized at one angle of regard it will be at once recognized in any other. 


\section{CLINICAL AND ANATOMICAL EVIDENCE}

We can tum next to the clinical and anatomical endence that is related to the question of equipotentiality in perception

The conclusions reached already are supported by the perceptual completions in hemianopia, described by Fuchs (1920) and Lashley (1941) When one occipital pole of the brain has been destroyed by injury, or temporarily loses its function in a migrame attack, the patient becomes blind in half the visual field. If a simple symmetrical object such as a solid white square or a billard ball is fixated in the midline, the patient nevertheless reports that he sees all of it That he does not, but instead completes in his perception what he really sees only half of, is shown by another fact If half the object is presented instead, in such a way that the missing half would have fallen in the blind side, the patient still "sees" the whole object

What is the basis of this completion? Koffka (1935) treats it as a further evidence of Gestalt principles-an instance of the operation of field forces. This, however, is precisely what it cannot be.

In two of Fuchs' cases at least (Fuchs, 1920, pp 424, 436) the gunshot wound that produced hemianopia must have destroyed one area 17. The bullet entered the skull in the midline at the occipital pole, and could hardly have destroyed the optrc radiatrons on one side without also destroying the visual cortex on that side But area 17 is the cortical tissue in which a dynamic completion, due to field forces, would have to occur, there is no isomorphism in the excitation conducted beyond 17-conduction is diffuse, and the topological relationship to the visual stimulus that is retained from the retina to area 17 is lost beyond it, so the field-force idea is applicable only at this level. The completion, then, is not the result of a field process.

In the case of migraine scotoma described by Lashley, the symptoms indicate that one visual area was nonfunctional during an attack But this again is the tissue in which interference patterns would have to orignate, to produce the perception of a complete object fixated in the center. According to the theory 
of interference patterns (Lashley, 1942a) the perception of a semicircle lyug immedutely to the right of the fixation point depends on the partucular interference pattern that is set up by that diagram in the left visual cortex A whole curcle, fixated in the middle, sets up two such interference patterns, one from each visual area. In the hemianopic, only one can occur, why, then, should the patient see a whole circle? The conclusion is
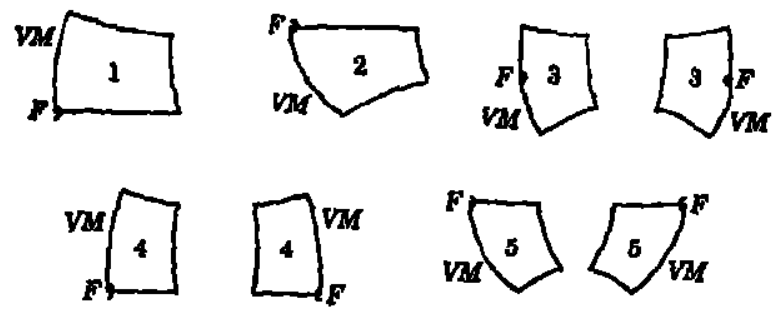

Ficores 4 Diagramming roughly the changes in cortical projection of a square when the fixation point only is changed based on the data of Polyak, 1941, and his figure 100, for a square subtending a visual angle of $18^{\circ} 20^{\prime}$ (the swze of the "central area" of the retuna) 1, fixation on the upper nght corner of the square, which thus falls in the lower left visual field and produces an excitation in the upper nght cortex only, 2, fixation on the lower right corner, 3 , bulateral projection with fixation on the center of the square, 4, bilateral, fixation on the midpoint of the top line of the square, 5 , fixation on midpoint of bottom line. $F$, projection of fixation pount, VM, vertical mendisn.

nevitable that the "completion" occurs in some other, undamaged, area of the cortex-and that it cannot, consequently, be a field process.

Next, consider some of the anatomical propertes of the visual cortex in which the field process must occur.

There is a point-to-point correspondence between the retina and the visual cortex, or area 17 (Lashley, 1934, Polyak, 1941). Thus there is an usomorphism of the visually stomulating surface with the resulting excitation in area 17 , and both the theories that we are examining assume that this fact accounts for the perception of a square, for example, as a distunctive whole But there are definte limits to what isomorphusm can account for in perception

Figure 4 represents roughly the patterns of cortical excitation 
that are aroused by lookang at different parts of the same pattern, a square These show how hard it is to suppose that the perception of a square, as distunct from other quadrulaterals, is determined by the shape of the isomorphic excitation in area 17, since that shape varies so much

(There are undoubtedly major errors in the figure, which is based on a diagram by Polyak [1941, figure 100]. Polyak made no atternpt to represent corresponding retinal and cortical points in detal, the necessary evidence for doing so is actually not in existence Polyak's diagram also deviates in some respects from his text, which is presumably the more accurate, his figure 100, for example, shows the "central fovea" half as wide as the "central area," whereas his text, pp. 230-231, suggests that the dufference in size is greater. Figure 4 follows his diagram, not the text. Finally, the human visual area cannot be represented accurately as a flat sheet )

Figure 4 is only roughly accurate, it seems certain, however, that the distortion, in the pattern of cortical excitation, that $\infty \mathrm{c}-$ curs when fixation is changed from one to another part of the figure is at least as great as that dragrammed If the data of Marshall and Talbot (1942, p 134) had been used ustead, the discrepancies would have been greater Furthermore, a difference in saze (in terms of retmal angle) between two squares would produce even greater changes in the shape of the cortical excrtation, although squares of different size are also supposed to denve their common identity from the distribution of excitathon in area 17.

The reproduction of retinal patterns in area 17 is topological, not topographical, and if identity were completely determined by the shape of the cortical excitation, regardless of what the fixation point may be, the perceived properties of a square with shifting gaze or at dufferent distances should change markedlyremaining, it is true, a quadrilateral (topological reproduction provides for this), but not retaning the same proportions. That is, errors should occur, in the perception of quadrilaterals, which do not occur, or in the perception of trangles, and so on. A crrcle in one angle of regard should be confused with an oval in another angle. One might postulate a differential cell density to correct the distortion of topographical relations which I 
have diagrammed, but there is no support from histological study of area 17 to support this ad hoc assumption, and I can find no way of dong it for one pattern which would not mean an even greater distortion for another.

However, another way of dealing with this obstacle for field theory has been proposed by Kohler and Wallach (1944), who suggest that a differential "satzation" or fatigue would correct the anatomical distortion of the cortical retina This proposal will be considered with other aspects of therr theory of figural aftereffects.

\section{THE THEORY OF FIGURAL AFTER-EFFECTS}

Kohler and Wallach (1944) have proposed a new treatment of perception which is in some respects very attractive and deals with a certain set of facts very efficiently On the other hand, I believe that the theory is inconsistent with some of the facts reported by Kohler and Wallach themselves, and that it cannot be considered to have disposed of the anatomical difficulty that has just been discussed.

Following some onginal experiments by Gibson, Kohler and Wallach have studied certain peculiar fatgue effects resulting from prolonged inspection of visual objects The method of experment is this The subject keeps his eyes fixed, for some two to three minutes, on a single "fixation point" in a large surface on which a diagram is also presented. The fixation point is determined by the expermenter so that the "inspection figure" stumulates a particular part of the retina. Then, after the inspection period, the subject looks at a second surface, again with a fixation point determined by the expermenter, which is placed so that a "test figure" on this second surface also stmulates a particular part of the retina Thus in figure 5 are represented both an inspection and a test figure, superimposed so that one can see the relationshup between the retunal projection of each. The fixation point is shown by a small $x$, the inspection figure (at which the subject stared first) is the large rectangle marked $I$, and the test figure is the set of four small squares each marked $T$. These four squares are so placed that two fall in a region of greater fatigue or "satiation," near the boundary of the 
rectangle, and two fall farther away, allowing one to discover the changes that occur in the neighborhood of the inspection figure, where satiation has occurred

When a test figure is projected on the retna near the place at which the inspection figure was projected, several effects can be observed in the test figure These effects include a color effect (fading), a depth effect (apparent increase of distance from the observer), and displacement effects (apparent change in direction of line, in posston, or in size) It is the displacement effects with which we are concerned here. Kohler and Wallach propose, first, that the basis of perception is the occurrence of electric currents aroused by the visual excitation in the cortex of area 17, secondly, that the flow of these currents, in the neighborhood of the excitation, increases the resistance of the tissue through which they flow This increase of resistance is satiation, and the changes it causes in current flow account for displacement effects. This is on the assumpton that perceived distance between two objects "varies with the degree to which corresponding cortical objects [i $e$,

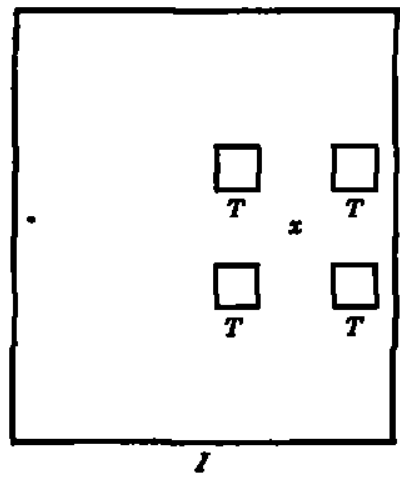

Figuri 5 Diagram from Kohler and Wallach, Proc Amer Phil Soc, 1944, 88, p 280 Courtesy of Amencan Philosophical Society and of the authors The large square, $I$, is the "inspection figure" to which the subject is first exposed, the large square is then removed, and the subject looks at the four smaller "test" squares, each marked $T$ the excitations in area 17] are interrelated by therr figure currents" (Kohler and Wallach, 1944, p 334) Just what this interrelation is and how it operates is not specified, but its degree decreases when the tissue between two areas of excitation has an increased resistance The two corresponding objects in the visual field then look farther apart. It is also important to observe that Kohler and Wallach state explictly (p 337) that apparent displacements are not dependent on actual changes in the locus of the excitations in area 17 only on an mcreased ressstance to current flow in the tissue between them.

These assumptions account economically for some of the phe- 
nomena described by Kohler and Wallach. Other phenomena, however, seem inconsistent with the theory Consider first the perceived size of a figure within an area of satiation, including the special case where the test figure comcides with the inspection figure According to the theory, the perceived size of a square or curcle should increase wh moreased satiation, since the greater resistance to current flow between opposite sides should produce an overestimation of the distance between them. In fact, the opposite is frequently reported by Kohler and Wallach when a test figure, such as square or circle, comcides exactly with the locus of the previously seen inspection figure, its apparent sye diminishes, Again, in figure 5, the excitations produced by the two small test squares on the right, near the boundary of the mspection rectangle, are separated by tissue that is more satiated than that separating excitations from the two small squares on the left-yet the two on the right appear closer together than those on the left. This difficulty for the theory is found in the data obtaned by Kohler and Wallach with their figures $1,2,3,4,5,7,10$, and so on

The problem has been recognuzed by the authors and is briefly discussed at the end of their monograph (pp 351, 356) They suggest two possible solutions one is that apparent size may be affected by the presence of a large area of satiation surrounding the figure, as well as by the degree of satiation withm it (p 351), another is the possibulity that an inmediate selfsatiation, by the test figure itself, may have a greater effect than immediate self-satiation plus a pre-exastent satiation (p 356). If I understand these explanations, they may undermme all the preceding argument, much as "afferent neural interaction" undermines Hull's theory by removing the definiteness of inference from his other postulates (p 15) Certanly these two influences, of immediate self-satiation and of a surrounding area of satiation, would have to be reckoned with in the production of other phenomena, else their existence would become an ad hoc postulate to be used only where difficulty appears This of course may mean only that the theory needs to be worked out in more detal, as Kohler and Wallach themselves have pointed out. 
Next, a difficulty of a somewhat different knd, related to the anatomical distortions of the cortical retina (the difficulty for configuration theory that was discussed in the preceding secton)

To deal with the anatomical fact that objects equally spaced in the visual field are not equally spaced for the cortical retma, the key assumption is made by Kohler and Wallach that a permanent satiation in the peripheral cortical retina would be greater than in the cortical fovea, and, accordingly, that anatomical distortion such as has been dragrammed in figure 4 would be corrected functionally The greater penpheral satiaton is thought to occur as follows Contours equally spaced on the retina would not be equally spaced in area 17 , because the peripheral retina has a smaller cortical representation. So an equal distribution of contours in the visual field, and consequently on the retina, would result in a bunching of contours in the peripheral part of the cortical retina, and a wide spacing of contours in the cortical macula On this argument, the closer spacing of contours would produce a higher level of satation in peripheral vision, which would compensate for the fact that a given retinal angle here is represented by a much shorter cor-. tical distance than the same angle in central vision (Kohler and Wallach, 1944, p. 345). Although the cortical distance is shorter, resistance is higher too so perceived distance remains the same.

But all this has overlooked an essential fact. Acuity in central vision is very much higher than in peripheral vssion, and so there is no basis for assuming that the central cortical retina would be the site of a smaller number of excitations per centmeter. Kohler and Wallach's argument assumes, essentally, that all contours in the visual field are supraliminal for the pernphery, given this assumption, their argument stands, but we know actually that the assumption cannot be made, that many contours are supralıminal for central vision and subliminal for peripheral vision These contours, sublummal at the penphery, would fill in the gaps between the more widely spaced excitations in central vsion that originate from contours which are supralıminal for the whole visual system, peripheral as well as central There is no evidence permitting one to be certain that the frequency of contours that are sublmmal for the peripheral field is such 
as to compensate exactly for the greater spacing of other contours in the macular area of the visual cortex, but what evidence we have implies this, since differences of acuity appear to be closely related to the rato of centmeters of cortex per degree of visual angle (Marshall and Talbot, 1942).

The theory of figural after-effects, therefore, has not avoided the difficulties for configuration theory that are to be found in the anatomy of the visual cortex.

\section{CONCLUSIONS}

The conclusions that have been arrived at concerning field theory and equipotentiality, in this and the preceding chapter, may be summarized as follows. The examination of these theones of perception was undertaken in the first place because no way could be found of dealing with attention and attitude, in - terms of the configurational hypothesis This still stands as an essential weakness, which by itself would justufy a search for some other approach to perception On closer inspection, however, one also finds that configuration theory has flaws even in the treatment of perception itself, and some of the flaws are serious It is not possible to reject the theory finally, for an answer may be found for each of the objections that have been rased, and the alternative treatment that is proposed in the two following chapters has its own difficulties and shortcomings-it is in fact because my theorzung about perception is in some respects implausible that I am obliged to emphasize weaknesses in other theones, just as later (in Chapter 8) a rather vague treatment of motivation is bolstered up by trying to show that Hull's alternative is not as precise as it seems

The fundamental difficulty with configuration theory, broadly speaking, is that it leaves too little room for the factor of expe-

' rience It makes difficulty in seeing how learning can occur (particularly, perceptual learning), and in seemg how autonomous central processes can exist and influence behavior Assocration (as distuct from conditioned reflex) theory, on the other hand, may make it possible to adopt a halfway position in which one can take advantage of some of the obvious values both of configurationist and of connectionist theories. 
It is important as psychology comes of age to avoid, if possible, the extreme positions that have often been adopted in the discussions of the past It is consequently relevant here to point out that a large part of the original thesis of the configurationusts (the Gestalt group, Lashley, Tolman, et al) has already been accepted and is fully made use of in current theorizing, and also that the theory to be presented in the following chapters is explicitly designed to deal from the first with the problems of form perception and attention or set-problems whose existence has been most insisted on in recent years by the configurationists-as well as those problems of learning and memory emphasized by learning theorists Thus the present argument is based at least as much on Gestalt as on learning theory.

The current debate between these two theories cannot be understood on the assumption that they are utterly opposed to each other It is rather to be seen as like the running battle between the Left and the Right in governmental policy. the Left contunually insisting on the inadequacy of present theory, workng to broaden and elaborate the conceptual armament of the experimenter, the Right msistung always on the dangers of being too free with changes that may open the doors to animism, on the importance of demonstrable evidence and intelligible definitions, and on the necessity of fully exploning the ideas we have now before rejecting them as insufficient. But in this conflict, as in the political one, it will be found over any great penod of time that the Right (in current psychology, learning theory) has adopted again and again the ideas originally advocated by the Left (currently configurationism), once they are properly aged and found not to have spoiled. Thus modern learning theory is "sophisticated", it deals freely in Ideas that were once anathema to it-1deas of gradients, generalizations, interaction among afferent processes as well as the classical afferent-efferent action, and so on Obviously both Left and Right are needed for the development of psychology, though it may be easy for the configurationist to forget the value of the systematzzing and search for rigor made by his opponent, or for the learning theornst to forget where some of his ideas originally came from. 


\section{The First Stage of Perception: Growth of the Assembly}

This chapter and the next develop a schema of neural action to show how a rapprochement can be mado between (1) perceptual generalization, (2) the permanence of learning, and (3) attention, determining tendency, or the like It is proposed first that a reperted stimulation of specific receptors will lead slowly to the formation of an "assembly" of association-area cells which can act briefly as a closed system after stumulaton has ceased; this prolongs the tume durng which the structural changes of learning can occur and constitutes the simplest instance of a representative process (mage or idea). The way in which this cell-assembly might be established, and its characteristics, are the subject matter of the present chapter In the following chapter the interrelationships between cell-assemblies are dealt with; these are the basis of temporal organization in central processes (attention, attitude, thought, and so on). The two chapters (4 and 5) construct the conceptual tools with which, in the following chapters, the problems of behavior are to be attacked.

The first step in this neural schematzing is a bald assumption about the structural changes that make lasting memory possible The assumption has repeatedly been made before, in one way or another, and repeatedly found unsatsfactory by the critics of learning theory I believe it is still necessary. As a result, I must show that in another context, of added anatomical and physsological knowledge, it becomes more defensible and more fertle than in the past.

The assumption, in brief, is that a growth process accompanymg synaptc activity makes the synapse more readily traversed 
This hypothesis of synaptic resistances, however, is different from earler ones in the following respects. (1) structural connections are postulated between single cells, but single cells are not effective units of transmission and such connections would be only one factor determining the direction of transmission, (2) no direct sensori-motor connections are supposed to be established in this way, in the adult animal, and (3) an intimate relationship is postulated between reverberatory action and structural changes at the synapse, implyng a dual trace mechanism

\section{THE POSSIBILITY OF A DUAL TRACE MECHANISM}

Hulgard and Marquus (1940) have shown how a reverberatory, transient trace mechanism might be proposed on the basis of Lorente de Nó's conclusions, that a cell as fired only by the simultancous activity of two or more afferent fibers, and that internuncial fibers are arranged in closed (potentally self-exciting) curcuits Their diagram is arranged to show how a reverberatory curcuit might establish a sensori-motor connection between receptor cells and the effectors which carry out a cond1toned response There is of course a good deal of psychological evidence which is opposed to such an oversimplified hypothesis, and Hilgard and Marquis do not put weight on it At the same time, it is important to see that something of the kind is not merely a possible but a necessary inference from certan neurological ideas. To the extent that anatomical and physiological observations establish the possibility of reverberatory after-effects of a sensory event, it is established that such a process would be the physiological basis of a transient "memory" of the stimulus There may, then, be a memory trace that is wholly a function of a pattern of neural activity, independent of any structural change.

Hugard and Marquis go on to point out that such a trace would be quite unstable. A reverberatory activity would be subject to the development of refractory states in the cells of the circuit in which it occurs, and external events could readily interrupt it We have already seen (in Chapter 1) that an "activity" trace can hardly account for the permanence of early 
learning, but at the same tume one may regard reverberatory activity as the explanation of other phenomena

There are memones which are instantaneously established, and as evanescent as they are immediate. In the repetition of digits, for example, an interval of a few seconds is enough to prevent any interference from one series on the next Also, some memones are both instantaneously established and permanent To account for the permanence, some structural change seems necessary, but a structural growth presumably would require an appreciable time. If some way can be found of supposing that a reverberatory trace might cooperate with the structural change, and carry the memory until the grouth change is made, we should be able to recognize the theoretical value of the trace which is an activity only, without having to ascribe all memory to it. The conception of a transient, unstable reverberatory trace is therefore useful, if it is possible to suppose also that some more permanent structural change remforces it There is no reason to think that a choice must be made between the two conceptions, there may be traces of both kinds, and memones which are dependent on both

\section{A NEUROPHYSIOLOGICAL POSTULATE}

Let us assume then that the persistence or repetition of a reverberatory activity (or "trace") tends to induce lasting cellular changes that add to its stability The assumption ${ }^{\circ}$ can be precusely stated as follows When an axon of cell $\mathrm{A}$ is near enough to excite a cell $\mathrm{B}$ and repeatedly or persistently takes part in firng $u t$, some grouth process or metabolic change takes place in one or both cells such that A's effictency, as one of the cells firing $\mathrm{B}$, is increased

The most obvious and I believe much the most probable suggestion concernung the way in which one cell could become more capable of firng another is that synaptic knobs develop and increase the area of contact between the afferent axon and efferent soma ("Soma" refers to dendrites and body, or all of

- See p 229 for a further discussion of this point and an elaboration of the assumption made concerning the nature of memory. 


\section{A Neurophysiological Postulate}

the cell except its axon) There is certanly no drrect evidence that this is so, and the postulated change if it exists may be metabolsc, affecting cellular rhythmicity and lumen, or there might be both metabolic and structural changes, mcluding a limited neurobiotaxis. There are several considerations, however, that make the growth of synaphic knobs a plausible conception The assumption stated above can be put more defnitely, as follows.

When one cell repeatedly assists in firing another, the axon of the first cell develops synaptuc knobs (or enlarges them if they already exist) in contact with the soma of the second cell. This seems to me the most likely mechanusm of a lasting effect of reverberatory action, but I wish to make it clear that the subsequent discussion depends only on the more generally stated proposition italicized above

It is wise to be explicit on another point also The propositon does not require action at any great distance, and certainly is not the same as Kappers' (Kappers, Huber, and Crosby, 1936) conception of the way in which neurobiotaxis controls axonal and dendritic outgrowth But my assumption is evidently related to Kappers' ideas, and not inconsistent with them The theory of neurobiotaxis has been severely criticized, and clearly it does not do all it was once thought to do On the other hand, neurobiotaxis may still be one factor determining the connections made by neural cells. If so, it would cooperate very neatly with the knob formation postulated above Criticism has been durected at the idea that neurobiotaxis $\mathrm{d}$ rects axonal growth throughout its whole course, and that the process sufficiently accounts for all neural connections The idea is not tenable, particularly in view of such work as that of Weiss (1941b) and Sperry (1943).

But none of this has shown that neurobrotaxs has no influence in neural growth, its operation, withen ranges of a centimeter or so, is still plausible Thus, in figure $\theta$ (Lorente de No, 1938a), the multiple synaptc knobs of fiber 2 on cell $C$ might be outgrowths from a fiber passing the cell at a distance, and determined by the fact of repeated simultaneous excitatrons in the two Again, the course followed by fiber 7 in the 


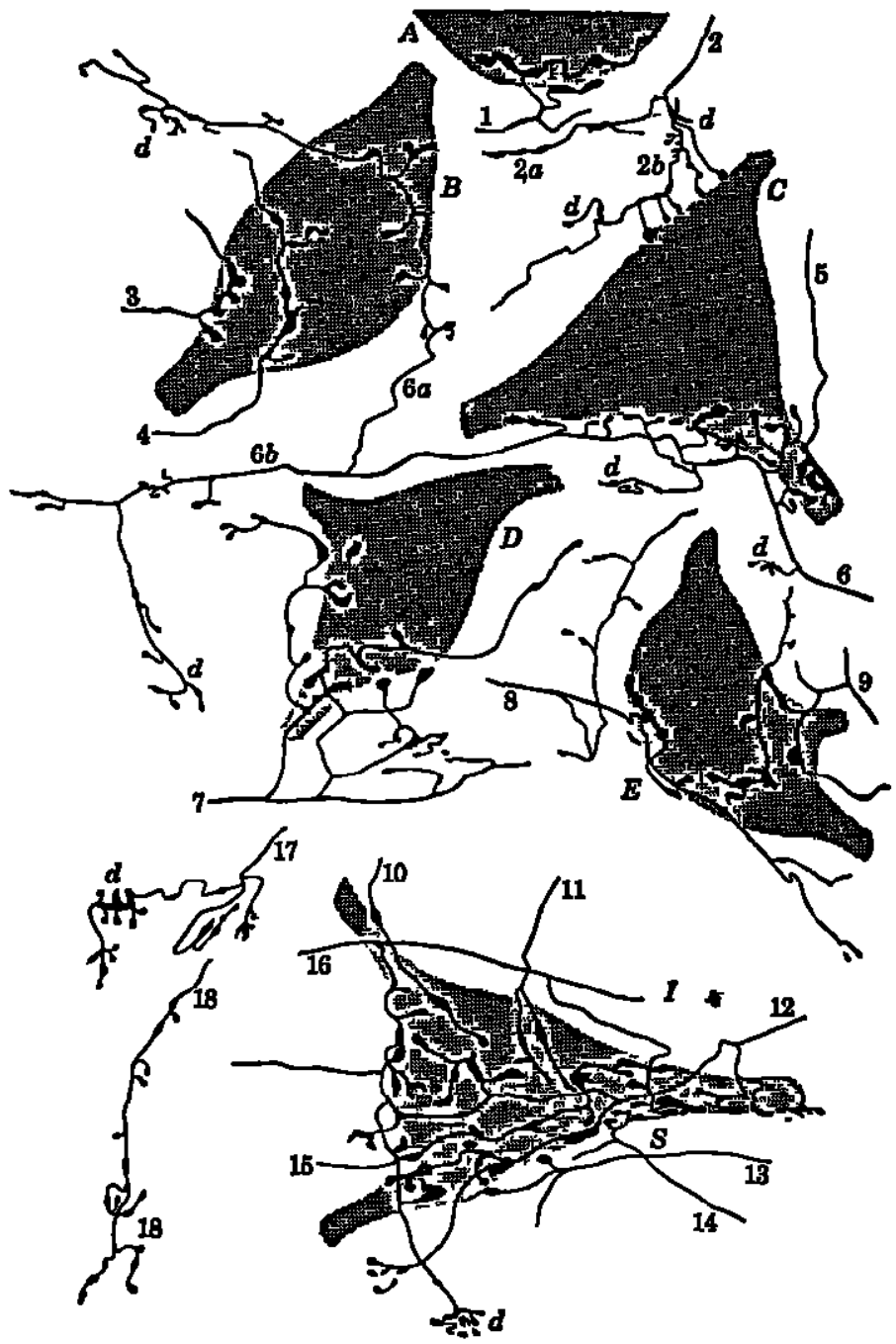

Ficurae 6 Relationships between synaptic knobs and the cell body From Lorente de Nó, 1988a Courtesy of Charles C Thomas and of the author 


\section{A Neurophysiological Postulate}

neighborhood of cell $D$ may include deflectons from the ongmal course of the fiber, determined in the same way.

The detals of these histological speculations are not important except to show what some of the possiblities of change at the synapse might be and to show that the mechanism of learning discussed in this chapter is not wholly out of touch with what is known about the neural cell The changed faciltation that constitutes learning might occur in other ways without affecting the rest of the theory To make it mare spectic, I have chosen to assume that the growth of synaptic knobs, with or without neurobiotaxis, is the basis of the change of facilitatron from one cell on another, and this 25 not altogether $1 \mathrm{~m}$ plausible It has been demonstrated by Arvanitak (1942) that a contiguity alone will permit the excitation aroused in one cell to be transmitted to another. There are also earlier exper1ments, reviewed by Arvanitak, with the same implication. Even more important, perhaps, is Erlanger's (1939) demonstration of impulse transmission across an artificial "synapse," a blocked segment of nerve more than a millimeter in extent. Consequently, in the intact nervous system, an axon that passes close to the dendrites or body of a second cell would be capable of helping to fire it, when the second cell is also exposed to other stimulation at the same point The probability that such closely timed coincidental excitations would occur is not considered for the moment but will be returned to. When the coincidence does occur, and the active fiber, which is merely close to the soma of another cell, adds to a local excitation in it, I assume that the joint action tends to produce a thickening of the fiber-forming a synaptic knob-or adds to a thickening already present

Lorente de No (1938a) has shown that the synaptuc knob is usually not a terminal structure (thus the term "end foot" or "end button" is misleading), nor always separated by a stalk from the axon or axon collateral If it were, of course, some action at a distance would be inevitably suggested, if such connections are formed in learning The knob instead is often a rather irregular thickening in the unmyelinated part of an axon near its ending, where it is threading its way through a thicket of dendrites and cell bodies. The point in the axon where the 
thickening occurs does not appear to be determined by tho structure of the cell of which it is a part but by something extermal to the cell and related to the presence of a second cell. The number and size of the knobs formed by one cell in contact with a second cell vary also. In the light of these facts it is not implausible to suppose that the extent of the contact established is a function of joint cellular activity, given propmquity of the two cells.

Also, if a synapse is crossed only by the action of two or more afferent cells, the implication is that the greater the area of contact the greater the likelshood that action in one cell will be decisve in firng another." Thus three afferent fibers with extensive knob contact could fire a cell that otherwise might be fired only by four or more fibers, or fired sooner with knobs than without.

In short, it is feasible to assume that synaptic knobs develop with neural activity and represent a lowered synaptic resistance. It is implied that the knobs appear in the course of learning, but this does not give us a means of testing the assumption. There is apparently no good evidence concerning the relative frequency of knobs in infant and adult brains, and the assumption does not imply that there should be none in the newborn infant. The learning referred to is learning in a very general sense, which must certainly have begun long before birth (see e.g, the footnote on pp 121-2)

- One point should perhaps be made explicat. Followng Lorente de N6,' two afferent cells are considered to be effective at the synapse, when one is not, only because their contacts with the efferent cell are close together so their action summates When both are active, they create a larger region of local disturbance in the efferent soma The larger the knobs in a given cluster, therefore, the smaller the number that might activate the cell on which they are located On occasion, a single afferent cell must be effective in transmission It is worth pointing this out, also, because it might appear to the reader otherwise that there is something mystenous about emphasis on the necessity of activity in two or more cells to activate the synapse. All that has really been shown is that in some curcurnstances two or more afferent cells are necessary Fowever, this inevitably imples that an increase in the number of afferent cells simultaneously active must increase the relability with which the synapse is traversed. 


\section{CONDUCTION FROM AREA 17}

In order to apply this idea (of a structural remforcement of synaptic transmission) to visual perception, it is necessary first to examine the known properties of conduction from the visual cortex, area 17, to areas 18, 19, and 20 (In view of the criticisms of architectonic theory by Lashley and Clark [1946], it may be said that Brodmann's areas are referred to here as a convenient designation of relative cortical position, without supposing that the areas are necessarly functional entities or always histologically distunctive.)

It has already been seen that there is a topological reproduction of retmal activities in area 17, but that conduction from 17 to 18 is diffuse Von Bonin, Garol, and McCulloch (1942) have found that a localized excitation in 17 is conducted to a large part of 18, a band lying along the margins of 17. There is no point-to-point correspondence of 17 and 18 Excitation from 18 is conducted back to the nearest border region of 17 , to all parts of area, 18 itself, and to all, parts of the contralateral 18, of area 19 (lying anternor to 18), and of area 20 (in the lower part of the temporal lobe)

The diffusity of conduction from area 17 is illustrated by the dragram of figure 7 Cells lying in the same part of 17 may conduct to different points in 18 The cells in 18, thus stimulated, also lead to points in 18 itself which are widely separated, to any part of the ipsiateral areas 19 and 20 , and, through one synapse, to any part of the contralateral 19 and 20 . Conversely, cells lying in different parts of 17 or 18 may have connections with the same point in 18 or 20

Thus there is convergence as well as spread of excitation. The second point illustrated by figure 7 is a selective action in 18, depending on the convergence of fibers from 17. In the figure, $F$ and $G$ are two cells in area 18 connecting the same macroscopic areas. $F$, however, is one that happens to be exposed to excitations from both $A$ and $B$ (two different regions in area 17). When an area-17 excitation includes both $A$ and $B, F$ is much more likely to be fired than $G$ The figure does not show the short, closed, multuple chans which are found in all parts 
of the cortex and whose faclitating activity would often make It possible for a single fiber from $B$ to fire $G$ But the same sort of local bombardment would also and in finng $F$, and the cell which receives excitations from two area-17 fibers simultaneously would be more likely to fire than that which receives excitation from only one

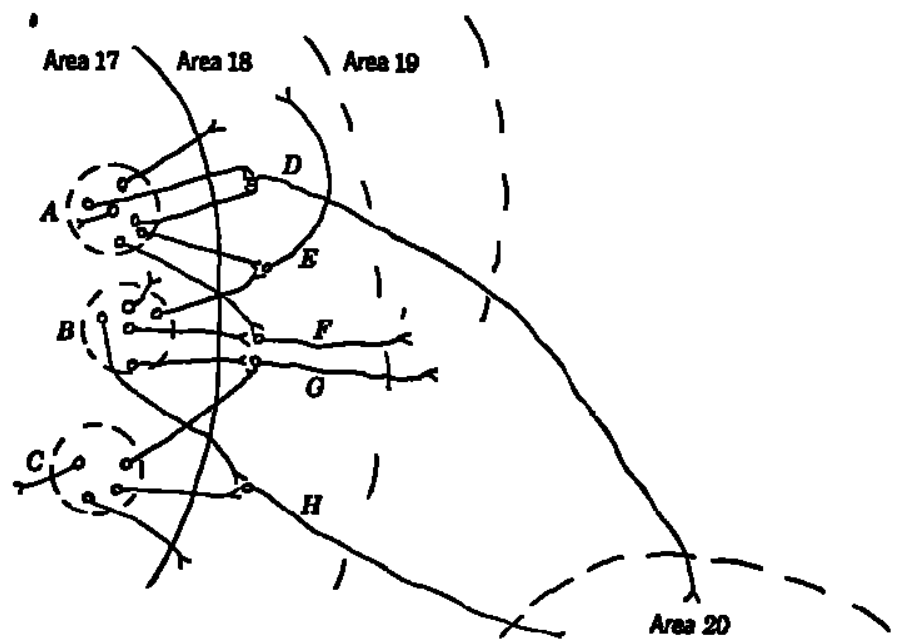

Figure 7 Hllustrating convergence of cells in Brodmann's area 17 upon cells in area 18, these cells in turn leading to other areas $A, B, C$, three grossly dustnct regions in area $17, D, E, F, G, H$, cells in area 18 See text

On the other hand, when $B$ and $C$ (instead of $A$ and $B$ ) are excited sumultaneously, $G$ would be more likely to fire than $F$ Any specific region of activity in area 17 would tend to excite specific cells in area 18 which would tend not to be fired by the excitation of another region in 17 . These spectic cells in 18 would be diffusely arranged, as far as we know at random They would be usually at some distance from one another and would always be intermingled with others which are not fired by the same afferent stimulation, but because of their lasting structural connections would tend always to be selectively excited, in the same combination, whenever the same excitation recurs in area 17 This of course would apply also in areas 19 and 20 Sunce a single pount m 18 fires to many pounts through- 
out 19 and 20, excrtation of any large number of area-18 cells means that convergence in 19 and 20 must be expected How often it would happen is a statistical question, which, will be deferred to a later section.

The tissues made active beyond area 17 , by two different visual stimuli, would thus be (1) grossly the same, (2) histologically distinct. A dufference of stumulating pattern would not mean any gross difference in the part of the brain which medrates perception (except in the afferent structures up to and including area 17, the visual cortex). Even a completely unilateral activity, it should be noted, would have diffuse effects throughout areas 18, 19, and 20 not only on one side of the bram but on both. At the same time, a difference of locus or pattern of stimulation would mean a difference in the particular cells in these areas that aro consistently or maximally fired.

\section{MODE OF PERCEPTUAL INTEGRATION THE CELL-ASSEMBLY}

In the last chapter it was shown that there are important properties of perception which cannot be ascribed to events in area 17, and that these are propertes which seem particularly dependent on learning. That "sdentity" is not due to what happens in 17 is strongly impled by the distortions that occur in the projection of a retinal excitation to the cortex. When the facts of hemianopic completion are also considered, the conclusion appears inescapable Perception must depend on other structures besides area 17

But we now find, at the level of area 18 and beyond, that all topographical organization in the visual process seems to have disappeared All that is left is activity in an irregular arrangement of cells, which are intertangled with others that have nothing to do with the perception of the moment We know of course that perception of simple objects is unified and determinate, a well-organized process. What basis can be found for an integration of action, in cells that are anatomically so disorganized?

An answer to this question is provided by the structural change at the synapse which has been assumed to take place in 
learning. The answer is not simple, perceptual integration would not be accomplished drectly, but only as a slow development, and, for the purposes of exposition, at least, would involve several distunct stages, whth the first of which we shall now be concerned.

The general idea is an old one, that any two cells or systems of cells that are repeatedly active at the same tıme will tend to become "associated," so that activity in one facilitates activity in the other The details of speculation that follow are intended to show how this old xdea might be put to work again, with the equally old idea of a lowered synaptic "resistance," under the eye of a different neurophysiology from that which engendered them. (It is perhaps worth while to note that the two ideas have most often been combined only in the special case in which one cell is associated with another, of a higher level or order in transmission, which it fires, what I am proposing is a possible basis of association of two afferent fibers of the same order-in principle, a sensori-sensory associntion," in addition to the linear association of conditioning theory )

The proposal is most simply illustrated by cells $A, B$, and $C$ in figure $8 A$ and $B$, visual-area cells, are simultaneously active The cell $A$ synapses, of course, with a large number of cells in 18, and $C$ is supposed to be one that happens to lead back into 17. Cells such as $C$ would be those that produce the local wedge-shaped area of finng in 17 when a point in 18 is strychninized (von Bonin, Garol, and McCulloch, 1942) The cells in the region of 17 to which $C$ leads are being fired by the same massive sensory excitation that fires $A$, and $C$ would almost necessarily make contact with some cell $B$ that also fires into 18 , or communicate with $B$ at one step removed, through a shortaxon crrcuit. With repettion of the same massive excitation in 17 the same firing relations would recur and, according to the assumption made, growth changes would take place at synapses $A C$ and $C B$ This means that $A$ and $B$, both afferent neurons

- It should be observed, however, that some theornsts have contanued to mantain that "S-S" (sensori-sensory) associations are formed in the learning process, and have provided experimental evidence that seems to estabhsh the fact. See, e g. Brogden, J Exp Psychol, 1947, 37, 527-539, and earler papers cated therein 
of the same order, would no longer act independently of each other.

At the same tume, in the conditions of stumulation that are dragrammed in figure $8, A$ would also be likely to synapse (drectly, or vie a short closed link) with a cell $D$ which leads back into an unexcited part of 17 , and there synapses with still

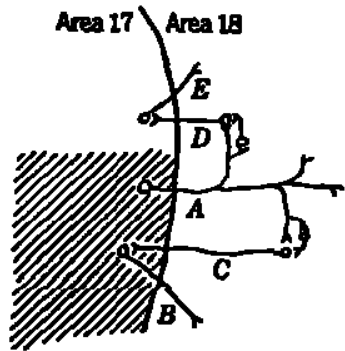

Fieures 8

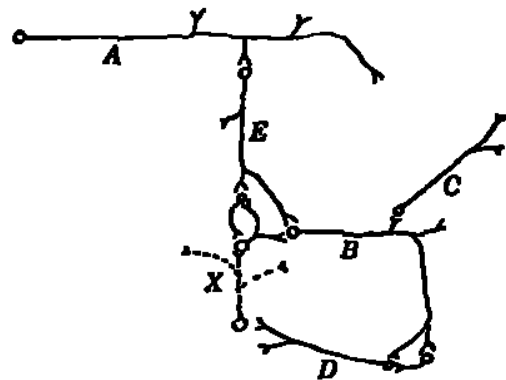

Ficure 9

Ficuria 8 Cells $A$ and $B$ hie in a region of area 17 (shown by hatching) which is massively excited by an afferent stimulation $C$ is a cell in area 18 which leads back into $17 \quad E$ is in area 17 but hes outslde the region of activity See text.

Fieure $\theta \quad A, B$, and $C$ are cells in area 18 which are excited by converging fibers (not shown) leading from a specific pattern of activity in area 17. $D, E$, and $X$ are, among the many cells with whtch $A, B$, and $C$ have connections, ones which would contribute to an integration of their activity. See text.

another cell $E$ of the same order as $A$ and $B$ The synapse $D E$, however, would be unlikely to be traversed, since it is not like $C B$ exposed to concentrated afferent bombardment. Upon frequent repetition of the particular excitation in area 17 , a functional relationship of activity in $A$ and $B$ would increase much more than a relatronshp of $A$ to $E$.

The same considerations can be applied to the activity of the enormous number of individual cells in 18, 19, and 20 that are simultaneously aroused by an extensive activity in 17. Here, it should be observed, the evidence of neuronography implies that there are anatomical connections of every point with every other point, within a few millimeters, and that there is no orderly arrangement of the cells concerned. 


\section{Growth of the Assembly}

Figure 9 diagrams three cells, $A, B$, and $C$, that are effectively fired in 18 by a partcular visual stumulation, frequently repeated (by fixation, for example, on some point in a constant distant environment). $D, E$, and $X$ represent possible connections which might be found between such cells, durectly or with intervening links. Supposing that time relations in the firing of these cells make it possible, activity in $A$ would contribute to the firing of $E$, and that in $B$ to fing $C$ and $D$ Growth changes at the synapses $A E, B C, B D$, and so on, would be a beguning of integration and would increase the probability of coordinated activity in each pair of neurons.

The fundamental meaning of the assumption of growth at the synapse is in the effect this would have on the timing of action by the efferent cell The mcreased area of contact means that firing by the efferent cell is more likely to follow the lead of the afferent cell. A fiber of order $n$ thus gams increased control over a fiber $n+1$, makung the firng of $n+1$ more predictable or determunate The control cannot be absolute, but "optnonal" (Lorente de N6, 1939), and depends also on other events in the system In the present case, however, the massive excitation in 17 would tend to establish constant conditions throughout the system durng the brief penod of a single visual fixation, and the postulated synaptic changes would also increase the degree of this constancy $A$ would acquire an increasing control of $E$, and $E$, with each repetition of the visual stmmulus, would fire more consistently at the same tme that $B$ is firing ( $B$, it will be recalled, is drrectly controlled by the area-17 action) Synaptic changes $E B$ would therefore result. Simularly, $B$ acquires an uncreasing control of $D$, and whenever a cell such as $D$ happens to be one that connects again with $B$, through $X$, a closed cycle (BDXB) is set up.

It 2 , however, misleading to put emphass on the coincidences necessary for the occurrence of such a simple closed circut. Instead of a ring or hoop, the best analogy to the sort of structure which would be set up or "assembled" is a closed solid cage-work, or three-dimensional lattıce, with no regular structure, and with connections possible from any one intersection to any other Let me say explicitly, again, that the specificity of such an assembly of cells in 18 or 20 , to a particular excitation 
in 17 , depends on convergences Whenever two cells, directly or indrectly controlled by that excitation, converge on another cell (as $E$ and $X$ converge on $B$ in figure 9 ) the essental condition of the present schematzzing is fulfilled, the two converging cells need not have any simple anatomical or physiological relation to one another, and phys1ological integration would not be supposed to consist of independent closed chains

This has an important consequence. Lorente de No (1938b) has put stress on the fact that actuvity in a short closed crrcurt must be rapidly extinguushed, and could hardly persist as long as a hundredth of a second It is hard, on the other hand, to see how a long, many-linked chain, capable of longer reverberation, would get established as a functional unit. But look now at figure 10, which dragrams a different sort of possib1lity Arrows represent not neurons, but multple pathways, of whatever complexity is necessary so that each arrow stands for a functional unit These units fire in

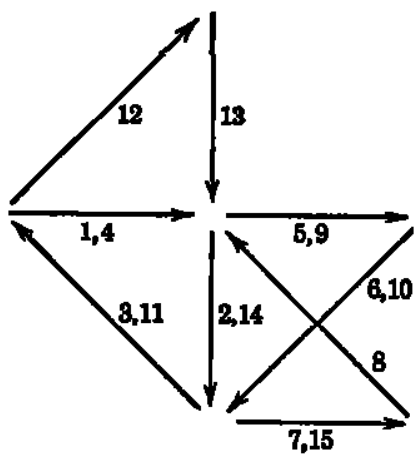

Figure 10 Arrows represent a simple "assembly" of neural pathways or open multople chains finng according to the numbers on each (the pathway " 1,4 " fires first and fourth, and so on), lllustrating the possibility of an "alternating" reverberation which would not extingursh as readily as that in a simple closed curcurt. the order $1,2,3, \cdots 15$ The pathway labeled $(1,4)$ is the first to fire, and also the fourth, $(2,14)$ fires second and fourteenth, and so on The actuvity 1-2-3-4 is in a relatively simple closed crrcuit. At this point the next unit $(2,14)$ may be refractory, which would effectively extingush reverberation in that simple crrcuit. But at this point, also, another pathway $(5,9)$ may be excitable and permit activity in the larger system to contunue in some way as that suggested by the numbers in the figure. The sort of irregular three-dimensional net which might be the anatomical basis of perceptual integration in the association areas would be infinitely more complex than anything one could show with a diagram and would provide a large number of the mult- 
ple parallel (or alternate) units which are suggested by figure 10. If so, an indefinte reverberation in the structure might be possible, so long as the background activity in other cells in the same gross region remained the same It would not of course remain the same for long, especially with changes of visual fixation, but such considerations make it possible to conceive of “alternating" reverberation which might frequently last for periods of tume as great as falf a second or a second.

(What I have in mind, in emphasizing half a second or so as the duration of a reverberatory activity, is the observed duration of a single content in perception [Pllsbury, 1913, Boring, 1933]. Attention wanders, and the best estumate one can make of the duration of a single "conscious content" is of this timeorder.)

Thus then is the cell-assembly. Some of its characteristios have been defined only by implication, and these are to be developed elsewhere, partucularly in the remainder of this chapter, in the following chapter, and in Chapter 8 (see pp. 195-7). The assembly is thought of as a system mherently involving some equipotentrality, in the presence of alternate pathways each having the same function, so that brain damage might remove some pathways without preventing the system from functioning, particularly if the system has been long established, with welldeveloped synaptic knobs which decrease the number of fibers that must be active at once to traverse a synapse.

\section{STATISTICAL CONSIDERATIONS}

It must have appeared to the reader who examned figures 8 and 9 carefully that there was something unljkely about its being arranged at the Creation to have such neat connections exactly where they were most needed for my hypothesis of perceptual integration The answer of course is statistical the neurons diagrammed were those which happen to have such connections, and, given a large enough population of connecting fibers distributed at random, the improbable connection must become quite frequent, in absolute numbers. The next task is to assess the statistical element in these calculations, and show that probability is not stretched too far. 
The diagrams and discussion of the preceding section require the frequent existence of two kands of coincidence (1) synchronization of firng in two or more converging axons, and (2) the anatomical fact of convergence in fibers which are, so far as we know, arranged at random The necessity of these coincidences sets a limut to postulating functional connections ad $h b$. as the basis of integration But this is not really a difficulty, since the psychological evdence (as we shall see) also imphes that there are limits to perceptual integration

Consider first the enormous frequency and complexity of the actual neural connections that have been demonstrated bistologically and physiologically. One is apt to think of the neural cell as having perhaps two or three or half a dozen connections with other cells, and as leading from one munute pount in the central nervous system to one other minute paint. Thus unpression is far from the truth and no doubt is due to the difficulty of representing the true state of affairs in a printed drawing.

Forbes (1939) mentions for example an estimate of 1300 synapte knobs on a single anternor horn cell. Lorente de Nó's drawings (1943, figures 71-73, 75) show a complexity, in the ramification of axon and dendrite, that smiply has no relation whatever to diagrams (such as mue) showing a cell with one or two connections The gross extent of the volume of cortex militrated by the collaterals of the axon of a single neuron is measured in millimeters, not in microns, it certainly is not a single point, microscopic in size In area 18, the strychnine method demonstrates that each tuny area of cortex has connectons with the whole region. (These areas are about as small as $1 \mathrm{sq} \mathrm{mm}$, according to McCulloch, 1944b) It puts no great strain on probabulities to suppose that there would be, in area 18, some anatomical connection of any one cell, excited by a particular visual stmulation, with a number of others excited in the same way.

There is, therefore, the anatomical basis of a great number of convergences among the multitude of cortical cells directly or indrectly excited by any massive retmal activity. This is to be kept in mind as one approaches the physiological question of synchronization in the converging fibers. In the tridunensional, lattice-like assembly of cells that I have supposed to be the basis 
of perceptual integration, those interconnecting neurons which synapse with the same cell would be functionally in parallel Figure 10 illustrates this The pathways labeled $(1,4),(8)$, and (13), converging on one synapse, must have the same function in the system, or the two-link pathway $(5,9)-(6,10)$ the same function as the single link $(2,14)$. When mpulses in one such path are not effectve, those in another, arriving at a different time, could be.

Once more, the oversimplification of such diagrams is highly misleading At each synapse there must be a considerable dispersion in the time of arrival of impulses, and in each individual fiber a constant variation of responsiveness, and one could never predicate a determinate pattern of action in any small segment of the system In the larger system, however, a statustical constancy might be quite predictable

It is not necessary, and not possible, to define the cellassembly underlyung a perception as being made up of neurons all of which are active when the proper visual stimulation occurs One can suppose that there would always be activity in some of the group of elements which are in functional parallel (they are not of course geometrically parallel) When for example excitation can be conducted to a particular point in the system from five different directions, the activity characteristic of the system as a whole might be mantamed by excitation in any three of the five pathways, and no one fiber would have to be synchronized with any other one fiber.

There would still be some necessity of synchronization, and this has another aspect. In the integration which has been hypothesized, depending on the development of synaptic knobs and an increasing probability of control by afferent over efferent flbers, there would necessarily be a gradual change of the frequency characteristics of the system The consequence would be a sort of fractionation and recruitment, and some change in the neurons making up the system That is, some units, capable at first of synchronzing with others in the system, would no longer be able to do so and would drop out "fractionation" Others, at first incompatible, would be recruited. With perceptual development there would thus be a slow growth in the assembly, understanding by "growth" not necessarily an increase 
in the number of constituent cells, but a change. How great the change would be there is no way of telling, but it is a change that may have importance for psychological problems when some of the phenomena of association are considered.

This then is the statistical approach to the problem It is drectly implied that an "association" of two cells in the-same region, or of two systems of cells, would vary, in the probability of its occurrence, over a wide range If one chose such pairs at random one would find some between which no association was possible, some in which association was promptly and easily established when the two were simultaneously active, and a large proportion makmg up a gradation from one of these extremes to the other The larger the system with a determmate general pattern of action, the more readily an association could be formed with another system On a statistical basis, the more points at which a chance anatomical convergence could occur, the greater the frequency of effective interfaclitation between the two assembles.

Psychologically, these ideas mean (1) that there is a prolonged period of integration of the individual perception, apart from associating the perception with anything else, (2) that an association between two perceptions is likely to be possible only after each one has independently been organized, or integrated, (3) that, even between two integrated perceptions, there may be a considerable vanation in the ease with which association can occur Finally, (4) the apparent necessity of supposing that there would be a "growth," or fractionation and recruitment, in the cell-assembly underlying perception means that there might be significant dfferences in the properties of perception at different stages of integration One cannot guess how great the changes of growth would be, but it is concelvable, even probable, that if one knew where to look for the ev1dence one would find marked differences of identity in the perceptions of child and adult

The psychological implications of my schematzing, as far as it has gone, have been made explicit in order to show briefly that they are not contrary to fact We are not used to thinking of a simple perception as slowly and painfully learned, as the present chapter would suggest, but it has already been seen, in 
the discussion of the vision of the congenitally blind after operation, that it actually is The slowness of learning, and the froquent instances of total falure to learn at all in periods as great as a year following operation (Senden, 1932), are extraordinary and incredible (if it were not for the full confirmation by Riesen, 1947). The prnciples of learning to be found in psychological textbooks are denved from the behavior of the halfgrown or adult animal. Our ideas as to the readiness with which assocration is set up apply to the behavior of the developed organism, as Bonng (1946) has noted, there is no evidence whatever to show that a simularly prompt association of separate perceptions can occur at burth-that it is independent of a slow process in which the perceptions to be associated must first be integrated

As to the wide range in difficulty of associatung two ideas or perceptions, even for the adult, this is psychologically a matter of common expenence Who has not had trouble remembering, in spite of repeated efforts, the spelling or pronunciation of some word, or the name of some acquaintance? The fact of the unequal difficulty of associations is not stressed in the literature, probably because it does not fit into conditioned-reflex theory, but it is a fact $M y$ speculations concerning the nature of the trace and the abongunal development of perception thus are not obvnously opposed to the psychological endence. Further evaluation can be postponed until the speculations have been fully developed. 


\section{Perception of a Complex: The Phase Sequence}

The reader will remember that what we are aiming at here is the solution of a psychological problem To get psychological theory out of a difficult umpasse, one must find a way of reconcling three things without recourse to animism. perceptual generalization, the stablity of memory, and the instabilities of attention As neurophysiology, this and the preceding chapter go beyond the bounds of useful speculation They make too many steps of inference without expenmental check. As psychology, they are part of a preparation for experument, a search for order in a body of phenomena about which our ideas are confused and contradictory, and the psychological evidence does provide some check on the inferences made here

Although this discussion is not anchored with sufficient neurophysiological evidence, it still has a compass It is guided throughout by the necessity of conforming to the concrete evdence of behavior, and any virtue it may have derives from coordnating the two sources of information The detals of these two chapters, speculative as they are, have to be given for two reasons.

First, competent psychologists have asserted that field or configuration theory is the only conceivable solution of the problems of behavior. The argument is stronger when a physiologist such as Sherrington (1941), whthout benefit of field theory, can find no possible way of reducing "mind"-which must be the control of behavior-to neural action. The argument is even strengthened by the vagueness of Hull's treatment of percepthon "afferent neural interaction" and generalzzation gradients have the smell of brimstone about them and, when they are 


\section{Perception of a Complex: The Phase Sequence}

made specific, in neurological terms, may turn out to be only a new form of field theory (just as Pavlov's waves of unhbition and excitation are field theory). Improbable as Kohler's theory of electrical fields may be, hard as it may be to reconcule Lashley's theory of interference patterns with the anatomical and clinical evidence, these writers still haye a powerful argument for holding psychology to what I believe is a blind alley, so long as it remains true that an alternative explanation cannot be elaborated in enough detal to be conceivably an account of real processes.

So one must speculate. But there is a second reason for the form of the discussion in these two chapters In speculating. one can at least be specific enough so that, when further anatomical and physiological unformation is made avarlable to the psychologist, the errors of earlier speculation such as this will be apparent at once, and the necessary changes clearly indcated.

In this chapter, accordingly, the new foundation for association theory is to be completed The preceding chapter dealt only with the effects of a single visual stimulus, with a constant retinal projection We can next ask how the several effects of a varable stmulation may be combined, or associated.

\section{LINES AND ANGLES IN PERCEPTION}

It has already appeared that the corners of a rectlinear figure are of special importance in first vision. The first crude perceptron of an object $m$ the visual field as distinctive, as having identity, seems to be related to the sharp inflection of contours and the drection of line. Senden describes the congenitally blind patient, after operation, as frequently not being able to distungush (for example) square from curcle, but when the distunction is made, it is through a search for corners, apparently as focr in an otherwise amorphous mass of light There is also evidence of an immediate distuction between narrow strups of light, when one is vertical and one horzontal in the visual field Here again the perception of identity in such objects is defectuve, since the patient is extremely slow to learn names for the 
two vasual patterns, although he can name horizontal and vertical at once by touch

From Lashley's experiments, it is also evident that line and angle dominate the rat's perception of patterns. We may therofore consider that these things are among the elements from which more complex perceptions develop. "Perceptual element" is meant to contrast sharply with the punctate sensory elements of classical association theory, on the one hand, and, on the other, the term does not imply that the perception of line and angle is fully innate There is a prolonged learning penod before these "elements" are promptly and clearly perceived, ether by rat or man.

The evidence on this point concerning man is to be found in Senden's (1932) compulation and has been cited earler, some evidence follows which indicates that the rat also has to learn to see lines distnctively.

Eighteen rats reared in complete darkness (Hebb, 1937a) were traned, following Lashley's procedure exactly, to discrimlnate horizontal from vertical lines The only comparable data for normal anumals are from Lashley (1938b), who gives a mean of 21 trials for normal learning of this discrimination The mean number of trals for the rats reared in darkness was 129 , sx times as great, with a range from 40 to 190 These animals had been tramed first to jump to an open window, and then to discrimunate black and white cards (as were Lashley's animals) before being tramed with the horizontal and vertical strations In subsequent tests they behaved like normal animals, showng that the slow original learning with striations was not due to structural defects.

It is to be observed, in this experiment, that the range of learning scores overlapped the normal range. Some anmals reared in darkness learned as quickly as some normally reared Also, in prelıminary experiments, two anımals showed a prompt but not completely consistent discrimination of horzzontal and vertical line. These facts fit with Senden's, for human subjects, very closely, they indicate that both rat and man have a crude inmediate perception of a difference bety

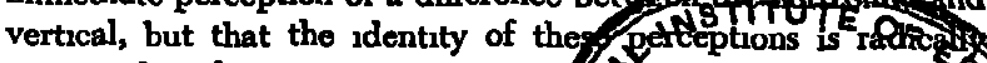
improved with experience. 
It may not be necessary to seek far for a physiological understanding of the primitive significance of line and the sharp inflection of line. In their stumulatmg statistical approach to the problem of visual acuity, Marshall and Talbot (1942) have discussed a mechanism of summation at the borders of the projection of a visual excitation in area 17 In the absence of other influence, the heightened activity corresponding to the margin of a figure might dommate the total process Walker and Weaver (1940) have shown a direct control of eyemovement by peripheral stumulation of the visual cortex. The movement is in such a direction that the light source which produced such a peripheral activity would be fixated. In vew of Walker and Weaver's work, Marshall and Talbot's discussion suggests that there would be a tendency to fixate, successively, various parts of the contour of an object Also, with the intersection of lines there may be a further summation of the border effects, so that the foci of greatest activity aroused by a pattern in the visual field would correspond to its corners

It is important to observe that the figure made up of straight lines, instead of irregular curves, has special physiological propertes. When one point of a straight line is fixated, every point to one side of the fixation tends to arouse exactly the same direction of eyemovement, and every point in the line on the other side exactly the opposite direction. At tmes the two vectors may balance, but often they will not. It follows that there is a strong tendency for the eye to make a sweep along the line, in one direction or the other, and, as the sweep is made, at the moment when a corner is reached the stimulation of the intersectung line is at the maximum, since at this moment every point in the second line has the same vector, for a new direction of eyemovement. It appears from these considerations that the eye would tend to seek out the contours of a figure and follow themirregularly, and with reversals, it is true, and subject to disturbance by other events, but it seems that such a tendency must exist.

It is also important to note that every movement of the eye in a single durection, for whatever reason, stumulates a "ridge" of activity in area 17 corresponding to the projection of a straight line, wherever the visual field contans a point of light. Except 
in the special case of a field consisting of uniform parallel striatons, every change of fixation from one point to another means that rows of cells in area 17 are excited together or left unexcited together. From the assumptions of the preceding chapter, the first visual learning would to a great extent be the integration of the effects, in the peristrnate region, of the action of such rows of cells in the strnate region This is a further emphasis on the importance of straight lines in perception, and, as I have argued (in Chapter 3), makes it possible to assume, from such a constant and extended training, that the characternstics of adult tachistoscopic vision may be accounted for by a learning process.

Lines and angles, then, can be treated as perceptual elements, not fully mnate in perception, but partly so, and lukely to be learned before more complex patterns are $A$ very incomplete analogy will serve here as a mnemonic device, to help keep in mind the different processes that enter into perceptual learning. If line and angle are the bncks from which form perceptions are built, the primitive unity of the figure might be regarded as mortar, and eyemovement as the hand of the bulder The analogy is poor because the bricks contain mortar, they grow while they are being used, and the house may change beyond recognition as it is being bult Nevertheless, two things must be kept in mind First, the primitive figure-ground relationship stressed by the Gestalt psychologasts remains of fundamental importance even though one recognizes that it is not the be-all and end-all of perception. Secondly, one may agree with Lashley and the Gestalt psychologists that motor activity in itself cannot possibly explain the organization of perception and nevertheless recognize that it has an essential role. Bricks alone are not enough for bulding, nor yet is mortar.

A triangle then is a complex entity in perception, not primitive As a whole, it becomes distunctive and recognizable only after a prolonged learning period in which there is a good deal of receptor adjustment-head-and-eye movement-as the psychological evidence of Chapter 2 showed From the point of view we have now arrived at, the difficulty of explanning the expernmental facts of pattern equivalence has been greatly reduced. Sinnlar trangles of unequal size, a soldd and an outline trangle, even a solid plain triangle and one curcumscribed with a curcle 


\section{Perception of a Complex. The Phase Sequence}

(Lashley, 1938b) contain a number of identical elements-lines and angles with the same onentation. (For some readers, "1dentreal elements" will be an mvitation to battle let me say again that these are not the sensory elements of the classical dispute )

It is of course stul necessary to show how these elements, the parts of the figure, are integrated in perception. This I shall try to do next, taking an example that is as simple as possible. The present schema of perception and perceptual learning deals only with the case in which changes of visual fixation and some locomotion occur freely The problem is to show how the variable stimulation which results from such movements can have a single effect, the perception of a sungle, determinate pattern

\section{MOVEMENTS DURING PERCEPTUAL INTEGRATION}

In the perception of a triangle, there are three nodal points in the pattern on which fixation would be made repeatedly $A$ single fixation point was assumed in discussing perceptual integration in the last chapter Let us next try to elaborate the schema to provide explicitly for movement of the eyes, and for those movements of the whole animal that produce vanations in the size of the retmal projection of the stumulating pattern

The account of neural integration now needs to be elaborated in three respects (1) The activity in the peristruate, temporal, and other association areas of the cortex, that is aroused by fixation on any one of the three corners of the thangle $A B C$ (figure 11), is always accompaned by motor processes which control the changes of fixatron to the other comers (2) Because of these changes, the integration of activity in area 18 and beyond occurs without constant conditions of excitation in area 17. when for example $B$ is fixated, which cells will be excited in 18 is determined in part by the preceding actinty in 18, and this vanes according to whether the preceding fixation was on $A$ or on $C$ (3) Fmally, each of the three retinal patterns produced by fixation on the corners of the triangle is itself variable, with different distances of the animal from the stimulus object

To show as simply as possible what these three points involve, suppose now that an anumal sees a trangle repeatedly, having had no prevous visual experience and having no other stimula- 
ton at the same time. Disregard any changes of background activity in the bram, apart from those induced by the changes of fixation mentzoned, and, as a final sumplification, treat the three angles $A, B$, and $C$ of figure 11 as the only perceptual elements unvolved: that 28 , disregard fixation on the sides of the trangle, and the percepton of the lines as distinctive entities The three changes to be made in the schema now can be dealt with one by one.

1 First, the concomitant mo. tor excitation with fixation on $A$, as represented in figure 11 , $B$ and $C$ falling outside the macular field, the peripheral stimulation of $B$ and $C$ excites two motor responses simultaneously. The excitation may of course be subliminal, particularly at the first moment of fixation on $A$, since a fixation often endures for an appreciable time The relative strengths of the motor components of the excitation aroused by $B$ and $C$ can be supposed to be the same, statistically, as

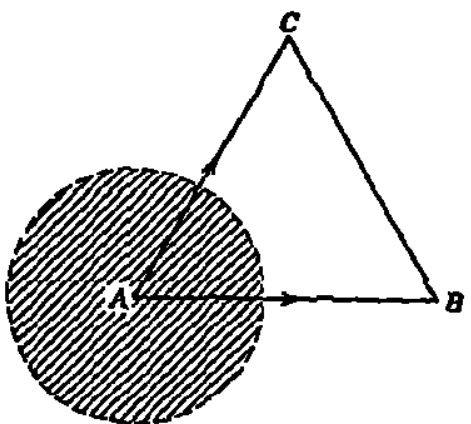

Ficura II The triangle ABC is seen with fixation on the point $A$ The macular field is represented by the hatched arrcle, so that the points $B$ and $C$ fall in penpheral vision Arrows represent the direction and strength of eyemovement tendencies aroused by the stumulatlon from $B$ and C shown by the vectors (the length of the arrows) in the figure At any one moment, variations of excitability would make one dommant over the other, but one can suppose that on the average they are equal. Looking at $A$ would be followed by looking at $B$ about as often as $C$.

In the normal adult animal, at least, the control of the drection and extent of eyemovement is accurate within quite narrow limits and does not work through a series of gross approxunations. This means that the neural activity leading to the motor conters, before an eyemovement begins, is determunate, and specific to the locus of the penpheral stimulus. Fuxation on $A$, then, has two effects' one "central" ( $i e$, the arousal of specific cells in the peristriate and temporal regions, without a drect 
motor significance), and one primarily motor ( $2 e$, the arousal of specific fibers in a pathway that is not known but which origmates in the visual cortex and which presumably mvolves the frontal eyefields [Clark and Lashley, 1947])

These two effects, central and motor, correspond roughly to a duchotomy of the total excitation in area 17 The central effects are mainly determined by the part of the excitation falling in the macular zone, where acuity is greatest and the motor components least, the motor effects, by the perpheral part of the excitation, where acuity is poor, the ratio of cortical cells to degrees of visual angle least, and the motor limen lowest (Walker and Weaver, 1940).

If the cells in area 18 and beyond which are aroused by fixaton on $A$ are designated by the letter $a$, the composition of $a$ is determined by the whole triangle, but the angle $A$ has a disproportionately great influence because it falls in the field of central vision Millimeter for millumeter, the part of the triangle which falls on the macula excites more cortical cells Thus the main determinant of the central effect of fixating $A$ is the angle $A$ itself, rather than $B$ or $C$ We shall also find that this influence of $A$ in organizang the neural structure $a$ must be further increased if the triangle is seen at a variable distance, but it is important to remember that $a$ is defined orignally as made up of the cells in the association areas of the cortex that are actvated, not by $A$ alone, but by the whole triangle when fixation is on $A$

In the same notation, $b$ and $c$ are aroused by fixation on $B$ and $C$ The distinction of $a$ from $b$ is a distinction of the exc1tations aroused with two retinal projections of the same figure In brief, the angle $A$ is the main determmant of the structure $a$, but not the only one, while $B$ and $C$, when $A$ is fixated, are the main determinants of the subsequent motor response

As the integration of $a, b$, and $c$ proceeds, repeated fixation on the three corners of the trangle slowly establishes three diffuse, urregular cell-assemblies (Chapter 4), each of which is capable of acting momentarily as a closed system. As this goes on, the activity of each structure would at all stages of its development be accompanied by two specific motor excitations Activity in $a$ occurs in the constant presence of a liminal or subliminal exc1- 
tation tending to move the eyes to the nght (fixating $B$, figure 11) or upwards and to the nght (fixating $C$ ) From the assumptons of the preceding chapter, this means some mutual facilitation between $a$ and the motor activity in question Psychologically, in the particular limited conditions of the schema, percoption of the triangle with fixation at one corner would involve a set to look at one of the other two corners (faclitation by $a$ of the motor responses, strengthening the motor faclitation from $B$ and $C$ ), and a sensorly aroused change of fixation from $A$ to $B$ would produce an expectancy of seeing the triangle with fixation at $B$ (facilitation from the motor activity, arousing or reunforcing activity in $b$ ) ${ }^{\bullet}$

2. The second pount (on whoch the schema as it appeared in

- That is, when the anumal looks at $B$ after lookng at $A$, the assembly $b$ is excited in two ways centrally, by the facilitation from $a$ and from the motor activity, and sensorily, by $B$ itself In these circumstances, the two faciltations would just about coincide in time "Expectancy" implies that the central facilitation definitely precedes the sensory, so it would be better to speak here of attention (central reinforcement of a sensory process) But the same process is called expectancy when the sensory reinforcement is delayed

The later chapters will consider the interaction of sensory and central facilitations, and the disturbing effect on behavior when they conflict or when an expectancy fals to get sensory renforcement In a thoroughly familar environment, the two facilitations would be synchronized, mainly because of the motor links in the central "phase sequence" discussed in the following pages The facilitation of $a$ is equally on $b$ and on $c$, so $a$ might arouse esther one But the cortical motor activity that results in lookang from $A$ to $B$ is specific to $b$, and the anumal will not "expect" to see the point $C$

None of these relationships can be so precise outside of this schema, but the prnciples can hold Central and sensory processes will stay more or less in synchrony in a familar environment, both because of the motor links in the central sequence, and because the facllitation of a perception $X$ will not be specific to another particular perception $Y$ unless $X$ was invanably followed by $Y$ in past expenence-the sight of an unsupported object, and the sound of a crash But a sequence of visual sensations which is not organized by one's own eyemovements can be very disturbing to behavior, as the occasional case of seasickness at the movies shows Again, the sight of a large body of calm water is not disturbing in a strange landscape, where one's expectancies are not specific, but would be very much so if it followed a movement of the head and eyes toward a place where one had always seen dry land These points are developed further in Chapter 7. 
the last chapter must be modified) has to do with an interference between the suprasensory effects of fixation on different points in the stmulating pattern

It was proposed that the cells which are excited in area 18, by the first visual stumulation, are determined by chance anatomical convergences Those which are active when the angle $A$ is looked at must, on a statistical basis, mclude some which are active when $B$ or $C$ is looked at. The number of convergences in common would also be increased by any overlap in the retunal projections of a figure Consequently, when $A$ and $B$, or $B$ and $C$, are looked at in close succession, there must be an influence of the one activity, in the peristrate region, on the next. When $B$ is looked at, some cells in 18 will be active if $A$ was looked at just before, but not if $C$ was, others mhibited, or refractory, following a look at $A$. In the early stages of the visual development, there must be an interaction between $a, b$, and $c$, which in this schema amounts to an interference In the ordinary course of perceptual learning, evidently, where the eyes are first opened on the complications of an ordinary room without the artficial limits of the schema, the amount of interference between the first perceptual activities must be much greater." The interference has to be fully recognized here if the schema is to have any theoretical value later.

Such an interference, it seems, can occur only in the first stages of perceptual development and would disappear later. It is important to recognize it, however, since it affects the course that integration takes In terms of the notation used above, the organization of the structure $a$ (excited by fixation on A) must proceed mainly in cell groups (effective units of transmission) which are reliably excited whenever fixation is on $A$, whether $B$ or $C$, or netther, had been fixated just before. I have

- And the resultant generalization of perception will be greater also In this schematzing, the conditions of vsual learning have been simplified to an absurd degree, but the ideas that emerge from it can be applied to behavior in more natural circumstances The inital interference between the three primary visual habits epitomizes a relationship between a much larger number of habits in real perceptual development The larger number of habits means that each one will be more an abstraction, less a perception of a particular angle (or line) in a particular setting 
emphasized that there must be fractionation and recruitment, or growth, as the individual closed cell-assembly becomes physiologically organized When the growth occurs in a variable background of visual stimulation, those cells which are affected by the variability would not be consistently active at each repetiton of the specific stimulus which excites the assembly, and therefore would not be part of it In other words, as growth proceeds in $a, b$, and $c$, fractionation would eliminate elements that are active only when a certan fixation has preceded, and those which are mactive following any particular fixation Activity in $a$ can therefore occur independently of $b$ or $c$, once the integration of these assemblies is complete

In general terms, what has just been discussed is the case in which sensory events recur in random order in a short time interval, as one must suppose visual fixations to do The alternative is a fixed sequence of sensory events, which of course also happens in normal perceptual development The difference between the two cases is instructive

Using the same notation, let us consider for a moment what would be the result of lookang at $A, B$, and $C$ always in the same order ( $A$ is now a "signal" for $B$, and $B$ for $C$, but this is not an analogue of the conditioned reflex. in the hypothetical conditions of the schema, $B$ occurs only following $A$, in the classical conditioning procedure, $B$ is an event such as feeding which follows $A$, the conditioned stmulus, but which also occurs at other tumes ) With a fixed order of stimulation, activity in the assembly $b$ occurs always in the same setting, the after-effects of activity in $a$ The structure $b$ therefore would include transmission units whose activity is especially facilitated by $a$, whereas, when stimulation occurs at random, $b$ must be made up of units that are independent of the preceding activity. Also, when actunty in $a, b$, and $c$ occurs always in that order, no reverberation in $a$ can be expected to coexust and be independent of activity in $b$, but must fuse with it. Any reverberatory after-effects which are not extinguushed by the changed sensory influx are constantly present during the physiological integration of $b$ and could not be distnct from it.

When fixation is at random among $A, B$, and $C$, however, we have seen that fractionation would tend from the first to keep 


\section{Perception of a Complex. The Phase Sequence}

the structures $a, b$, and $c$ distinct, and make activity in $b$ possible without preceding facilitation from $a$ This leaves the possibility that the three assemblies, once integrated, could still develop the interfacilitation that is discussed below, while the three are independent in the. sense that one can be active apart from another, or that two may be active at the same tme

3 The third point, on which the schema as it was developed in the last chapter is to be elaborated, concerns the effect of a variable distance of the animal from the stimulus object The result must be an increased importance of the part of the pattern which falls in the field of central vision.

When an animal looks at any part of a rectilinear pattern while moving toward it, the excitation aroused by the part looked at, falling in central vision, remains constant The rest of the excitation, in the peripheral field, varies steadily If the point of fixation is, say, the midpoint of one of the sides of the figure, the locus of the retnal projecton of that line does not change, except to be extended at each end If an angle of a triangle is fixated, as in figure 11, the projection of two lines and their intersection in the macula is unchanged as the animal moves closer to the figure, the third line, and the two remaining intersections, move farther and farther toward the periphery of the visual field

The rat in the Lashley jumping stand, learning to discriminate a square from a trangle, does not see the figures at a constant distance The retinal angular extent might vary by 50 per cent The varability is very much greater in a Yerkes box, where the animal runs toward, or may back away from, a door on which the stimulus figure is placed Now, using the same notation, in the composition of the assembly $a$ (the organized structure in the association areas of the brain which is aroused by looking at the point $A$ in figure 11), the influence of the angle $A$, relative to $B$ and $C$, becomes even greater The two lines and their intersection, fixated at a variable distance, have a constant effect, the other two intersections have a variable effect and do not repeatedly excite the same cells in area 18 In the growth of $a, b$, and $c$, fractionation would eliminate most of the cells whose excitation is due to the extramacular part of the figure This excitation would of course always coexist with the activity 


\section{The Spatial Hypothesis}

of the organized assembles, but it would remain unorganized The organized activity would have to be determined by the macular excitation, which, with appropriate fixation, is constant despite a varable angular stze of the strmulus object This conclusion is clearly significant in understanding stumulus equivalence, as demonstrated by a rat's choice of a large trangle after learning to choose a small one

\section{THE SPATIAL HYPOTHESIS AND PERCEPTION OF THE REMOTE ENVIRONMENT}

My discussion is already getting pretty far from the actual known facts of neural function If it is not to become fantasy, it must at least stack close, at every point, to the facts of behavior Here I digress for a moment to consider a group of facts that are well known but have not been explamed They serve as a check on the present theorizing

The preceding discussion implies that a variable stimulation from the same stimulus object would retard the rate at which the subject could learn to perceive it de novo Some of the discussion has suggested that differences of size, in terms of retinal angle, might not greatly affect learning, but differences of pattern, as an irregular object is seen from different directions, would be more important Each grossly different pattern of stimulation, as the object is seen from one side or another, requires the establishment of a separate set of cell-assemblies I can anticipate the discussion of the following section, and add that when this happens the vanous sets of assembles would graduglly acquire an interfacilitation-1f sight of the object from one angle is often followed by sight of it from another Arousing one set would then mean arousing the others, and essentrally the same total activity would be aroused in each case

Each perception would thus involve a conceptual activity (an activity, $i e$, not directly controlled by sensory processes) There is plenty of evidence in chldren's drawings, and in adult errors in perspective drawing, to show that a person looking at an object thinks he sees more of it than he does What he knows about the object appears in his drawing, as well as what is visible at the moment, and the significant fact is that neither 


\section{The Spatial Hypothesis}

of the organized assembles, but it would remain unorganized The organized activity would have to be determined by the macular excitation, which, with appropriate fixation, is constant despite a varable angular stze of the strmulus object This conclusion is clearly significant in understanding stumulus equivalence, as demonstrated by a rat's choice of a large trangle after learning to choose a small one

\section{THE SPATIAL HYPOTHESIS AND PERCEPTION OF THE REMOTE ENVIRONMENT}

My discussion is already getting pretty far from the actual known facts of neural function If it is not to become fantasy, it must at least stack close, at every point, to the facts of behavior Here I digress for a moment to consider a group of facts that are well known but have not been explamed They serve as a check on the present theorizing

The preceding discussion implies that a variable stimulation from the same stimulus object would retard the rate at which the subject could learn to perceive it de novo Some of the discussion has suggested that differences of size, in terms of retinal angle, might not greatly affect learning, but differences of pattern, as an irregular object is seen from different directions, would be more important Each grossly different pattern of stimulation, as the object is seen from one side or another, requires the establishment of a separate set of cell-assemblies I can anticipate the discussion of the following section, and add that when this happens the vanous sets of assembles would graduglly acquire an interfacilitation-1f sight of the object from one angle is often followed by sight of it from another Arousing one set would then mean arousing the others, and essentrally the same total activity would be aroused in each case

Each perception would thus involve a conceptual activity (an activity, $i e$, not directly controlled by sensory processes) There is plenty of evidence in chldren's drawings, and in adult errors in perspective drawing, to show that a person looking at an object thinks he sees more of it than he does What he knows about the object appears in his drawing, as well as what is visible at the moment, and the significant fact is that neither 


\section{Perception of a Complex The Phase Sequence}

chrld nor adult can usually say where his drawing departs from what is actually presented to the sense organ

An object seen from various aspects, then, requires a more complex learning process for its recognition than one seen always from the same direction and the same distance Such learning should take longer, and, if we assume that a smaller number of cortcal cells means a greater limitation to the number of assemblies that can be set up as separately functioning systems, learn.ing to recognize a variably stmulating object should be harder for lower animals than recognizing a constant object

Now it happens that the visual activity of lower species is dominated by the perception of place This turns out experimentally to mean a dominance of cues from remote objects $\mathrm{in}$ stead of near ones, and remote objects provide the most stable and constant stimulation of the anmmal's environment

In discrimination training, the "position habit," which Krechevsky (1932) has called a "spatial hypothesss," is a constant nusance in anmal experiments The method of training is to offer a choice of two alleys, or doors or windows, one of which leads the anmal to food $\mathrm{He}$ is also given a sign as to which one contains the food the correct door has a black card on it, or a curcle, say, the wrong one has a white card or a square Food, and the sign of food, are sometmes on the right, sometimes on the left But what the rat, dog, or chimpanzee persistently tres to find out is somethmg different He wants to know whether food is always on the right, or always on the left, or perhaps, in desperation, whether it alternates, once right, once left, and so on He wants nothing of the rarefied intellectual problem of the signs the expenmenter has put on the doors, it is only after repeated discouragement of the position habitthe attempt to find the food in some one place-that one can get him to learn anything else

It has sometimes been supposed that this is a visual lack, or that the vision of lower anjmals is dominated by other senses. But this interpretation is not right, for two reasons First, the position habit is just as annoying when one is trying to get blind animals to discriminate tactual cues (Smith, 1939) or auditory ones (Pennington, 1938). Secondly, the perception of place itself is visual, as much as kanesthetic or tactual When visual 
cues from the distant environment are avalable, they dominate behavior So the difference between lower and higher species is that the lower species is more dominated by the least variable of the environmental stimulation, not that vision in general has less influence.

This can be demonstrated strikngly in the rat. The rat is first accustomed to feeding on a bare table $\mathrm{He}$ is then taught to run across the table, from a fixed startung point, to a food dish that is always in the same place on the opposite side of the table The table is then rotated through $90^{\circ}$, but nothing else is changed. the rat is dropped at the same place on the table, with the food still opposite hum He runs at least once to the side of the table where the food used to be, with respect to the room, although netther food nor food dish is there now

If one changes the method slightly, the domnance of room cues as against table cues becomes clearer Put four small shields on the table, one in the middle of each edge Mark the one that contains food by panting it white and makng it larger than the others Train the rat first to run to that one only, by putting no food in the others Then rotate the table through $90^{\circ}$, and put food in all four shields The rat will choose, not the planly marked sheld he was taught to go to, but the unmarked shield which, after rotation, now occupies the place in the room that the food used to occupy (Hebb, 1938a, 1938b).

A still more strikng demonstration has been mentioned by Lashley (1938b) and further investigated by several students of Queen's University (Hebb and Willarns, 194I, and further unpublished experiments). Teach a rat to jump from a small platform to another one near by The second platform is just large enough for hrm to land on safely, and holds food After he has made ten jumps, move the second platform through $90^{\circ}$. The rat hesitates, shows disturbance, but finally jumps-into space, in the former durection of food.

A final demonstration that vsual cues are controlling the response can be made in a small cabinet, 6 by 6 feet square and 6 feet high, in a bulding quiet enough to provide no auditory cues to direction Each wall is identical with the others, and each contains a door. The ceiling is homogeneous One door is opened only, and a then curtain is hung over it to admit light 


\section{Perception of a Complex The Phase Sequence}

without allowing the animal to see outside. Now the rat is again taught to jump from one platform to another, in this cabmet When he has learned to jump, the door that was opened is closed, and one in the next wall, at a $90^{\circ}$ angle, is opened, and screened as before Nothing is changed but the more distant visual cues of the door, the nearby platform remains as it was, but the rat now jumps out into space, at a $90^{\circ}$ angle from his goal

Why? And why is the position habit so persistent? The answer seems to be that the animal manly percerves, and responds to, the least-variable objects in his environment, which are the ones at the greatest distance

The stumulation received from any object varies with the an1mal's movements, but there are important differences that depend on the distance of that object. When the anumal turns round, excitation is changed equally from near and far objects, but not when he moves from one place to another Changes of position affect retınal locus, extent, and intensity only slightly when the stumulus is remote, very greatly when it is near Even with body rotation, the order in which nearby objects are seen has no constancy, unless the animal always turns at precisely the same point. But the order in which distant objects are seen is the same, no matter where the animal is in the experimental apparatus

It seems therefore to be true that, the more constant the stimulation from an object is, the more readily it will be identrfied and responded to, as the schema of these chapters requires This is relevant also to the fact that as one goes up the phylogenetic scale one finds an moreasing tendency toward visual discrimination of objects, without special training The rat shows no sign of distmguishing between persons, or between mert objects visually presented, the dog often distunguishes a number of objects by vision, and probably a few persons, the chimpanzee is very selective in his behavior toward a great many objects, and is clearly able to distinguish a large number of persons (whom he sees often) from strangers

The fact therefore is that perception of the intrinsic visual properties of a near or movable object is a less primitive and more difficult feat than perception of its place This too is rele- 
vant to the fact that spatial delayed response is obtaned easily in monkey and chimpanzee, nonspatial only with the greatest difficulty (Riesen and Nissen, 1942)

My account in these pages evidently has a long way to go before the generalized human concept of a triangle, or any other figure or object, is provided for, but it is essential to remember that the rat's perception is also a long way removed from man's He does not recognize the triangle be was trained to recognize if it is rotated by $60^{\circ}$, nor a black triangle on a white ground after learning a white one on a black ground He has, in fact, some considerable difficulty in seeng the difference between a triangle and a square when their base-lines are identical. Normal man can recognize simple regular figures at a single glance, but it is very unlikely that a rat does When some change in the traming figure is made, such as making it larger, the rat's behavior is clearly disturbed, whle man might be quite unaware of the change, and the rat often discriminates only part of as simple a figure as a triangle

None of these differences between rat and man is accounted for in current theories of perception The critic of the account of perception that is offered here is likely to find it distasteful because it implies that with limited visual experience perception would be little generalized. Clinical and experimental evidence, however, does not support the objection but shows that the characternstic human perception of a triangle is the product of a long experience Thus the schema agrees with fact on this point and in addition has the advantage of suggesting, at least, why the perception of place and direction is so prominent in animal behavior.

\section{THE DEVELOPMENT OF SUPERORDINATE PERCEPTIONS}

We can now turn to the question of an integration of the several parts of a figure into a distinctive whole, as contrasted with the amorphous whole that is perceived in first vision

The most direct way of accounting for the superordinate integration is as follows, as long as this is still recognized as frankly schematic Activity in the assembly $a$, aroused by fixation on 


\section{Perception of a Complex. The Phase Sequence}

an angle $A$ of a triangle, can occur independently of $\boldsymbol{b}$ or $\boldsymbol{c}$. When $A, B$, and $C$ are looked at successively, in any order, but in a short period of time, activity may continue by reverberation in two of the structures while the third is sensorily aroused Just such a series of fixations would be the result of the behavior described by Senden, as the congenitally blind patient after operation learns to count the corners of a square or triangle and becomes quicker and quncker at doing so before he learns to recognize the figure at one glance. In these curcumstances, conceivably, there is a frequent occurrence of activity in the three assemblies $a, b$, and $c$ at the same tume. These he interlaced with each other in what is grossly the same tissue of the cerebrum, and according to the assumptions of the last chapter the simultaneous activity would result in an integration of the three systems.

It is perhaps necessary to remind the reader that the three systems do lie in the same tissue, although two of the sensory excitations concerned are unlateral, one in one striate area exclusively, one in the other All such suprasensory systems must develop in parallel, in both hemispheres The purely sensory activity, up to and including area 17, 1s unilateral when the stmulating diagram falls wholly to one side of the fixation point But when area 18 is strychninized, it fires into the contralateral 18 as well as mto the ipsilateral 19 and 20 (von Bonu, Garol, and McCulloch, 1942). The suprasensory integration initated by a unilateral sensory event must be bilateral and consist of two halves, in the two hemispheres Each half has the same functional significance-that $2 \mathrm{~s}$, it mediates the same perception and factitates the same responses-even apart from a coordinatung action of the corpus callosum and other cerebral commissures (though the development of the contralateral half depends on the commissures) This is evidently relevant to the clinical and experimental reports of slight effects from damage to the callosum (Bridgman and Smith, 1945), or failure to find an effect of unlateral extrpation of association areas, but it is important for the present as showing that the assemblies a (excited by a figure which falls wholly in the right homonymous visual field) and $b$ (arising from the left visual field) are structures which must develop in the same gross tissues of the brain 
According to the assumptions made earher, simultaneous actinty in $a, b$, and $c$ would establish faclitation between them, through their chance anatomical interconnections and the enlargement of synaptic knobs An effective facilitation from one system on another means a change of frequency characteristics in the system receiving the faciltation. It therefore means some fractionation and recrutment in the constituent units ( $p$ 76). With three extensive systems involved, each facilitating action in the other two, these growth changes must be considerable The resultung superordmate system must be essentrally a new one, by no means a sum or hookng together of $a, b$, and $c$ Instead of $a b c$, which might suggest such an idea, a better notaton for the new structure is $t$ the assembly of cells whose activity, in the schema, is perception of the trangle as a distinctive whole As Gestalt wnters would say, this is something other than the sum of its parts, but, unlike Gestalt theory, the schema derives the distinctiveness of the whole from perception of the parts.

Now for the defects in such a formulation Supposing that the general idea is right, it is still unlikely that the synthesis of $t$ from $a, b$, and $c$ would be made as a single step, however gradual A single step would depend on sumultaneous activity in three systems and so requires a frequent fixation of the three corners of the triangle in quick succession Looking at two of the corners only would not contribute to the integration

A much more plausible idea is that one or more intermediate stages would occur, such as an integration of $a$ with $b$ before that of " $a b$ " with $c$ ( $a b$ is used for brevity, though it is misleading in the same way as $a b c$ in the preceding paragraph) This would call for simultaneous activity in only two systems at a time I mention $a b$ as the first stage, rather than $a c$, because the honzontal line $(A B$, in figure 11) seems of fundamental importance in human perception, and certainly is so for the rat in the usual conditions of testing The perception of lines as distinctive entities has been dusregarded in this schema, in order to avoid a cumbrous and unwieldy discussion, but as we have seen the perception of lines is primitive, as the perception of angles or corners $1 s$, and a triangle has sux instead of the three perceptual elements dealt with by the schema This would 
make probable the occurrence of several intermediate stages between the perception of "elements" only, and perception of a distmctive total figure

The argument up to this point can be summarized in general terms Reasons have been given for believing (1) that fixation on each of the several parts of a figure would have an increasingly determinate effect, as arousing one specific structure, (2) that these structures, each corresponding to a frequently made fixation, are anatomically diffuse and interlaced with one another in the same gross cerebral tussue, and (3) that the several actunties may coexist, and be aroused in any order It is a reasonable inference (4) that two of these determmate actions simultaneously would have a determinate effect, tending to excite specific transmussion units, and that the action of these units would tend to organize in the same way that the earlier established systems were organized. Activity in a superordinate structure (in this case, $t$ ) is then best defined as being whatever determinate, organized activity results from repeated activity in the earlier-developed or subordinate structures giving rise to tt (1n this case, $a, b$, and $c$, or ${ }^{*} a b^{\circ}$ and $c$, assuming two steps in development, and that $a$ and $b$ are first integrated).

\section{THE PHASE SEQUENCE IN PERCEPTION}

Next, let us consider the temporal relationshup of actuvity in these vanous structures Durng the development of the assemblies $a, b$, and $c$, arousal of $a$ as we have seen is accompanied by two motor actuvities Of these, one always becomes liminal (producing a change of fixation) before $b$ or $c$ is sensorily aroused The sequence of events can be schematnzed as

$$
a-b-c-b-a-c-a-b-a-
$$

and so on. Each of these events is associated with two specific motor excitations One of them at least is subliminal, and one becomes liminal as an event intervening between $a$ and $b$, for example, or between $c$ and $a$

This "1deational" series with its motor elements I propose to call a "phase sequence"

When the assembly $t$ has become organzed, the psychological 


\section{The Phase Sequence in Perception}

endence indicates that its actinity intervenes between the activities of the subordinate assemblies $a, b$, and $c$ and does not supersede them. The sequence now becomes something like this.

$$
a-b-t-a-c-t-c-t-b-
$$

Such complication of a simple perception has important consequences for theorizing The reader is briefly reminded of the reasons for thinking that perception of a simple pattern is not a sngle lasting state, terminated by an external event, but a sequence of states or processes The congenitally blind patient after operation at first sees any figure as an amorphous mass, but may be able with effort to count its corners, the perception is then alternately of the whole and of its parts As the figure becomes a distunctive whole, there is still the same fluctuation of the figure-ground relationship-attention directed now to the whole, now to its parts. This is a phenomenon which as a matter of common observation is always present in percepton (and in a "concept," as one thinks about an object), as Chapter 2 showed, although the fact is not recognized in current discussions of the flgure-ground relationship Exactly the same sort of thing is implied by Lashley's inference that the rat successively isolates ( $i e$, sees as figure) various parts of a unfied pattern before makng a response

In terms of the schema, the alternate perception of whole and parts is an alternation of activity among $a, b, c$, and $t$, with corresponding directions of fixation (except for the entity $t$, which is accompanied by no determinate eyemovement, since the average values of the sux eyemovement vectors associated with the three part perceptions of the trangle add up to zero, but also fluctuate from moment to moment, their resultant would fluctuate in direction and amount, and would produce nether a fixation of gaze nor any predictable change of fixation).

It follows that the integration of $t$, the basis of perceiving a distinctive total figure, essentially involves a sequence of cortical events with motor components Activity in $a$ faciltates the arousal of both $b$ and $c$, with the appropriate intervening eyemovement, and activity in $b$ or $c$ facilitates the arousal of $a$ in the same way Whether $b$ or $c$ is aroused following $a$ would 


\section{Perception of a Complex The Phase Sequence}

depend on the momentary conditions of excitablity. Activity in $a$ would also faciltate that in $t$ In the early stages of perceptual development, $t$ might be excited only after repeated activations of $a, b$, and $c$, but later (with the extensive development of synaptic knobs in the system and the consequent increase in the strength of facilitation) might be aroused following sensory activation of $a$ alone, so that the triangle would be recognized with a single glance at $A$ (figure 11) But the activity so aroused must be transient, as we have seen; perception of the whole as such is momentary, and alternates with perception of the varous parts. Instead of an indefinitely prolonged reverberation, interrupted only by some event outside the system, excitation in one of the assemblies $a, b, c$, and $t$ is an unstable equilibnum which moves readily into another phase

The schema that has been developed requires only that reverberation contunue in one of these structures long enough so that temporal overlap can occur The psychological evidence reinforces the idea, based on the physiological evidence, that reverberation is short-lived if the duration of an idea, or a perception, is the duration of reverberatory activity in a closed system, one can say that the pattern of activity rarely lasts without change for as long as a second The stability of a perception is not in a single persistent pattern of cerebral activity but in the tendency of the phases of an irregular cycle to recur at short intervals.

It will be proposed in the following chapter that the train of thought is also a "phase sequence" of the same knd, but more extended, consisting of a senes of phase cycles eThe present discussion, besides dealing with perception, is also meant to lay a groundwork for dealing with the temporally organized processes of thinking.

\section{THEORETICAL PROPERTIES OF THE SCHEMA}

With this we are done schematzing It remains to ask what theoretical significance the schema has, and how its ideas are to be apphed to the development of behavior in normal circumstances

Actually, all the rest of thus monograph is devoted to answer- 
ing the questions, but it will be worth while first to strke 2 trial balance and see what has been accomplished already. The main conceptions used in the following chapters have now been developed The relationship of the schema to the following chapters will be more easily kept in mind by making its psychological reference more explicit.

Withn limits imposed by the needs of exposition, a conceptual system has been elaborated which relates the individual nerve cell to psychological phenomena A bridge has been thrown across the great gap between the detals of neurophysiology and the molar conceptions of psychology The bridge is definitely shaky in the middle, but it is well buttressed at each end, and we have a psychological bridgehead which can be widened and which already includes some strategic points In other words, the schema has some theoretical value already. It shows, more or less explicitly, how it is possible (1) to conceive of a conjoned action of the primitive figureground mechanusm, eyemovement, and learning (specifically defined synaptic changes), in the development of simple perceptions, (2) to provide for an action of set, attention, or expectancy, also defined physiologically, in the perceptual process, and (3) to provide at the same tume for Gestalt completion, sumilarity, generalization, and abstraction-these, with attention also, being essentally different aspects of the same process and closely related to association itself.

1 The interrelationships of eyemovement, figure-ground segregation, and learning are explicit in the schema Perception depends on leafnung first to see the parts of an object clearly, a process involving a series of visual fixations, and proceeds from seeing, at first, an amorphous mass containing several foci (the comers), to seeing a dustunctive figure at a glance. Even at this final stage we know that perception of the whole is dependent on eyemovements for maximal clarity (Chapter 2) According to the schema, the perception is constituted by a temporal sequence of activity in suprasensory (or association-area) structures, which owe their organization to changes at the synapse. it is an irregular cycle of recurring events which can continue momentarily without the corresponding sensory stumulations, but 


\section{Perception of a Complex: The Phase Sequence}

which is reinforced by them and by the appropnate eyemovements.

2. References to set, attention, and expectancy were made in developing the schema, which can be more precise here The term "attention" is ambiguous in the literature, and has several meanings it may refer ( $a$ ) to the state or end result of attending-the subjective clarity of what is attended to, or the necessary receptor adjustment, $(b)$ to the selectsvity of the process, (c) to the hypothetical agency or process which produces the selectuvity, and $(d)$ to vanous properties of "mind" which apparently cannot be defined or understood. It is in the third sense $(c)$ that the term is used here, and in the schema attenton may be defined as a central facilitation of a perceptual activity. So used, "attention" has exactly the same meaning as a "perceptual set," a process which makes one thing seen more readuly than another When this facilitation is effective in advance of the corresponding sensory process, expectancy is sand to occur

In the notation of this chapter, activity in the cell-assembly a facilitates an arousal of the assemblies $b$ and $c$, as well as faciltatung two motor responses, to fixate $B$ or $C$ (points of the triangle $A B C$, figure 11). In one way or another, this facilitaton has mherent in it the notons of assocration (the whole schema makes this explucit), attention, and expectancy. Actnvty in $b$ may be aroused in two ways. sensorly, by lookung at $B$, or centrally, by the "association of ideas." When $B$ is looked at just after $A$, actrvity in $b$ is aroused in both ways, and the central facilitation, from $a$, is an instance of attention a central reinforcement of a particular sensory event.

This illustration of the physiological meaning of attention is complete logically but is not particularly effectave, since it does not make the selectuvity of the process explicit Our hypothetical animal has not been allowed to see a variety of patterns as yet, among which selectivity could operate, but this will be clearer in a moment, in discussing abstraction. Sumlarly, an eyemovement from $A$ to $B$ may be aroused in two ways: by the - sensory stımulation from $B$, or by the faclitation from $a$ on the motor system Here the selectivity of the central facilitation is more evident. Eyemovements may be made from $A$ to $B$ or 
from $A$ to $C$, and which of these is made evidently depends often on central events We have seen that the relative strength of the two motor faclutations from $a$ fluctuates from moment to moment, when one becomes limunal-that is, becomes decisive in determining which eyemovement is made-the selectivity of the "motor set" is llustrated.

3 Suppose now that our hypothetical animal, after thorough habituation to hus limited environment ("limited" puts it muldly,

(C)

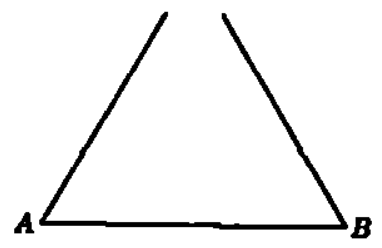

Ficure 12 The trangle of figure 11, lacking its apex.

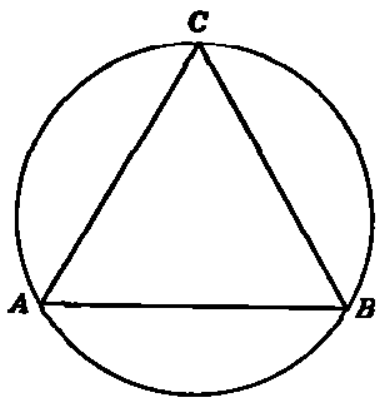

Ficure 13 The thangle of figure 11, circumscribed by a circle

since he was permitted no expenence except to see a single triangle, at varying distances but always with the same angle of regard)-suppose that the anmal is now tested with the two patterns represented in figures 12 and 13 Let him first see figure 12, a triangle such as he is used to, but with apex missing. What neural processes will result, according to the schema?

Fuxation near the top of the figure will have no organized effect (remember that the schema disregarded perception of lines as distunctive entities, discussing the intersection of lines only). If the anmal looks at $A$ and $B$, however, the assemblies $a$ and $b$ are excited, with facilitation on each other, on $c$, and on $t \quad A$ momentary excitation in $t$, a perception of the whole triangle, is then possible. Here is an instance of Gestalt completion, but derived as an associative process, with no field forces operating. According to the schema, it could happen only with a simple and thoroughly familiar figure (or thoroughly famliar part of a complex and unfamilar figure), which agrees with the 


\section{Perception of a Complex. The Phase Sequence}

experimental facts It is evident that such a completion becomes a simple corollary of association, once we can provide for a perception of parts without denymg the unity of the whole

So too are similarity, abstraction, and generalization. Given perception of the various parts or properties of a pattern, separately, and the possibility of an association of these perceptions with a perception of the whole we have given at once the possibility of a single response to two patterns which differ in their total propertes but which have a property or properties in common. This of course is not new What is new in the schema is showing how, concervably, perception of part and of whole can be related to each other and to the nerve cell, and to changes at the synapse An immediate dividend is the possibility of clearly stating, in physiological terms, the meaning of words like abstraction or simlarity which are necessary to describe behavior but which have had, to say the least, a touch of mystery about them

When the anumal whose perceptual processes have been schematuzed looks at the point $C$ in figure 13, at the apex of the familiar triangle, the intersection of the two straight lines produces an organized neural activity, plus an unorganized one due to the unfamllar presence of the curcumscribing crrcle In the notation of the schema, $c$ is aroused, but also a new and random actuvity of cells in area 18 and beyond To mterpret the effect of the random firing in 18 we must fall back on the statistical ideas of the last chapter The number of convergences of this new activity on the organized cell-structure $c$ would vary from anumal to animal, since it is a chance matter, and in any one anumal, from moment to moment, with variations of excitability in the individual unit of transmission Accordingly, the organzed activity in the assembly $c$ would sometimes be disrupted, sometimes not So with activities of $a$ and $b$, when the other corners of the triangle are fixated.

When the organized activity does occur, the triangle is "recognized"-the similarity of the figure to the familiar plain triangle (figure 11) is percelved, the anmal has abstracted from the total complex, and generalization of perception has occurred. Here too the selectivity of attention is more evident once $c$ is aroused, a fixation immediately afterward on $A$ will be more 
likely to arouse the organized activity of $a$-the sensory effect of the triangle is selectively renforced, by the facilitation from $c$, that of the crrcle is not

The purpose of this schema has been to arrive at conceptions that will be as near physiological fact as possible and that will enable us to deal with the cerebral control of behavior and its temporal integration From this point onward, it is assumed that some such structure as the cell-assembly is established as the result of repeating any particular finng combination in an afferent system, and that assemblies interact in some such way as that proposed in the schema

What follows is an attempt to generalize the ideas that have been developed in these two chapters (4 and 5), rather than an attempt at rigorous explanation by means of the assumptions that have already been made Two implications of those assumptions, however, may be made explicit, since they broaden the applucability of the schematizing to real problems

1. The different properties of a sungle sensory event may have separate central effects This can be conceived of as follows: The infant's hand makıng contact with two or three objects in succession may receive from them stmulations which differ except in one respect, such as the degree of pressure on the skin as the hand closes. In the schematizang, there was only one stimulating object in the environment, in the environment of an actual infant, stmulation involves a number of objects and a cumulative effect of stimulation would be established most promptly by the property that several of these objects have in common Such a consideration makes abstraction fundamental in the first learnug, thus, in the context of one group of stimulatung objects, a metal bar on the crib might contribute only to the development of the perception of hardness, but, while contact is being made intermittently with another set of objects, the same bar might contribute to the perception of (tactual) noundness

2 Another aspect of sensory processes can be taken account of, in the temporal sequence of stimulating conditions The problem of the perception of black in the visual field, for example, comes from the fact that this is not a static perception determined only by an absence of light in part of the visual 


\section{Perception of a Complex The Phase Sequence}

field, but is transient and requires temporal as well as spattal contrast for the full effect. A frequently repeated condition in the optic system is a declune of activity up to and moluding the lateral geniculate. During such a decline, and determined by It, there may be a predictable and selective activity in areas 17 and 18, by cells which are not active in the same combination during equilibration or during an mcrease of intensity. The repeated activity of such a particular group of cells would develop an assembly whose activity would be the perception of black

In general, then, whenever a change of stumulation produces a transient but selective activity (that is, m certain cells only), in the sensory projection areas or in the neighboring association area, the necessary conditions are provided for the formation of a cell-assembly Such assemblies would be the basis for perceiving contrasts, and the relative properties of stumulation of colder, larger, higher in pitch, and so on By the very conditons determining their occurrence, such perceptions must be transient and brief but may nonetheless be definite and distinctive for the penod of their duration. 


\section{Development of the Learming Capacity}

In the schema of percepton, motor learning got a foot inside the door through the emphasis on eyemovements. These movements were treated as having a vanable relationshup to sensaton They may be elicited (1) directly, by stimulation of the peripheral retina, (2) less durectly, by a foveal stimulus that arouses an assembly action, this in turn faciltating eyemovement, or (3) st.ll less drectly, through a phase sequence, or succession of assembly actions. The stimulus may arouse assembly $a, a$ then arousing $c$, at which point the motor limen may be low enough so that the activity of $c$ results in overt eyemovement

This epitomizes the treatment to be made of motor learning The behavioral evidence shows a considerable variation in the directness of senson-motor control Some responses have, at maturity, all the properties of a reflex and yet are known to be learned Others remain quite unpredictable from a knowledge of the stumulus alone, they are, that $1 \mathrm{~s}$, determined by an interplay of sensory stmulation and the autonomous actinty of the cerebrum (set).

We must remember both knds of learning the set-mfluenced and the non-set-influenced The reaction against early switchboard theory and connections, and the current dogma that learning occurs only with special conditions of motivation, have both tended to draw attention away from a kund of learning that, once establsshed, is little affected by set and does not seem to need reinforcement.

In the adult anmal the eyeblink to a rapidly approaching object, for example, is practically in the class of a spinal reflex 


\section{Development of the Learning Capacity}

Unlike the learned response studied in the usual experimentel approach, it is extremely resistant to extmction, and to distraction ("external inhubition"), and, in human subjects, very hard to change by verbal preparation Yet Riesen (1947) has shown that it is in fact a learned response It is absent at the twentieth month in the chimpanzee reared in darkness, but then appears slowly with visual expenetice, and it is found at about three months in the normally reared animal Such learning must be thought of as depending essentrally on sensori-motor connections The connections are presumably not from specific receptors to specific effectors, but they must be rather direct between afferent and efferent systems, since the response is without important influence from the autonomous central process (set, attention, or the like).

The responses that have this property, however, appear to be acquired early. They are by no means a paradigm of learning in general.

At another extreme are those learned acts of the half-grown or full-grown anmal that we call both learned and "voluntary" (a term that will be defined later) They are completely subject to extunction, and no matter how thoroughly learned they remain completely a function of set and drive There is no possiblity of direct sensori-motor connections here, to understand such behavior, and such learning, we shall first have to understand the organization of the controlling cortical activity

It is implied that the nature of the learning process changes significantly with development, and the epplication of the ideas of the schema of perception to the real behavior observed in an experiment will best be seen in considering first the changes of the learning capacity with growth

- Which means that the learned eyeblink may be about as close as mammalian learning ever gets to setting up S-R (stimulus-response) connectons The experimental criterion of such a response is dual its independence from set and attention, and its resistance to extrnction in Pavlov's sense This comes down essentully to a single theoretical cnterion the predictabulity of the response to a particular stumulation, in any circumstances, as long as no other physically incompatible response is aroused at the same time This is what sensord-motor connections imply But when a response is predictable only in the experimental apparatus, or following verbal preparation, we are dealing with another class of behavior 
It was possible to be specific about a hypothetical perceptual learning as it is not possible about the motor learning of the mature animal Perception is firmly tacked down to known stimulatung conditions, its antecedents can be discovered Motor learning at maturity, however, is conditioned by all the unknowns of cortical action. But with the schema as a starting point, and considering some properties of behavior that are often overlooked, it will be possible to set up a tentative account of learning at maturity.

\section{THE RELATION OF EARLY TO LATER LEARNING}

In this section I shall bring together the behavioral evidence on the relationship between learning in infancy and that of the normal adult animal, before returning in the next section to the question of neural mechanisms

It is proposed that the characteristics of learning undergo an important change as the animal grows, particularly in the higher mammals, that all learnung tends to utilize and buld on any earlier learning, instead of replacing it (Mowrer, 1941), so that much early learning tends to be permanent (Tinbergen, 1942, Hunt, 1941), and, finally, that the learning of the mature animal owes its efficiency to the slow and inefficient learning that has gone before, but may also be limited and canalized by it.

THE GENERAL PROPOSITION It is of course a truism that learning is often influenced by earlier learning Innumerable exper1ments have shown such a "transfer of training" Learning $A$ may be speeded up, hindered, or qualitatively changed by having learned $B$ before The question for debate is how great the effect may be in general behavioral development (as distmct from the effect of some one specific habit on some other) and what theoretical use is to be made of it.

McGeoch," for example, has sard.

After small amounts of learning early in the Iffe of the individual, every instance of learning is a function of the already learned organzation of the subject, that is, all learning is influenced by transfer ...

-The psychology of human leamtng, copynght Longmans, Green \& $\mathrm{CO}_{0}$. Inc, 1942, pp 445-446 By permission of the publushers 


\section{Development of the Learning Capacity}

The learning of complex, abstract, meaningful materials and the solution of problems by means of ideas (reasoning) are to a great extent functions of transfer Where the subject "sees into" the fundamental relations of a problem or has insight, transfer seems to be a major contributang condition It is, likewise, a basic factor in onglnality, the ornginal and creative person having, among other things, unusual sensitivity to the applicability of the already known to new problem situations Perceiving, at whatever level, is probably never free of its influence, and there is no complex psychological event which is not a function of it

Those are strong words, and I propose that they must be taken literally-as presumably they were meant to be taken Unless we are to regard them as just a lip service to logic and the known facts of behavior, they must influence general psychological theory profoundly If the learning we know and can study, in the mature animal, is heavily loaded, with transfer effects, what are the properties of the orignal learning from which those effects came? How can it be possible even to consider making a theory of learning in general from the data of maturity only? There must be a serious risk that what seems to be learning is really half transfer We cannot assume that we know what learning transfers and what does not for our knowledge of the extent of transfer is also denved from behavior at maturity, and the transfer from infant experiences may be much greater and more generalıed

An example, in itself important for the theory of learning, will also show the dangers of generalızing from adult to infant behavior with regard to transfer A student once pointed out to me that James' famous experment on memonzation begged the question James wanted to see if practice in memorization would increase the ability to memorize $H e$ found it did not, and later writers have found the same thing As a result, it has been concluded that practice por se has little or no transfer value, without instruction as to better methods of learning Büt all this is done with adults who have had long practice already, and the student pointed out that the transfer effects must have been complete before the experiments began, and could not be demonstrated by the method used James (1910) used highly educated adult subjects, Woodrow (1927) college sophomores 
What would have been the result whth subjects who had done no memorization before? We do not know, but it is certanly quite illogical to conclude that undurected practice has no transfer value because we find no evidence of it where there is no reason for expecting it Above all, it would be illogical-to conclude from this sort of evidence that the incidental learning of infancy has a negligible effect on later learning.

It has already been emphasized that perception is affected by past experience (Gibson, 1929, Carmichael, Hogan, and Walter, 1932, Leeper, 1935, Zangwill, 1937, Krechevsky, 1938) What is learned is in terms of what is perceived what is not perceived can hardly be remembered. Koffka (1935) has emphasized that patterns may be seen and remembered by the arousal of "older trace systems", Woodworth (1938) says that all perceiving is "schema with correction," that is, in terms of earler perceptual habits How do these habits get established in the first place? What are the properties of the learning that sets up the older trace systems," of learning in its first stages, before there are any earler habits to help it along? These questions cannot be completely answered at present, but even the skimpy evidence we have is enough to reonent the whole problem of learning $\checkmark$ THE EIRST LEARNING OF PFIMATES IS EXTREMMELY SLOW, AND VERY DIFFERENT FHOM THAT AT MATURrIY There are two kinds of learning. One is that of the newborn infant, or the visual learning of the adult reared in darkness or with congenital cataract, the other that of the normal adult. I have repeatedly cited the behavior of the patient born blind and given his sight after motor (and speech) development was well along, to show that the first learning is extremely mefficient as far as detectable effects on behavior are concerned, despite the completion of physical maturation (Senden, 1932) Here it is referred to once more, partly to show that the early mefficiency is not due to poor motivation and partly to make a comparison of man whth other species

Senden reports a serious disturbance of motivation, apparently in all cases, at one stage of leaming But this cannot be the main cause of the slowness of learnmg, for two reasons

1 Motivation is not disturbed in the first stage, immediately upon beginning to use the eyes At first there is a period of 


\section{Development of the Learning Capacity}

delight, particularly in colors, and apparently a complete preoccupation with the new experience.

Before long, the patient finds out how hard it is to get an effective use of pattern vision. The "crisis" of motivation then ensues Until that point there is interest and application, things that are easy to learn are learned and not forgotten, color names are readily remembered, but it takes a long apprenticeshup before any useful or demonstrable learning occurs in pattern vision.

Learning is evidently going on in this period, as long as the pathent continues to keep his eyes open and makes any effort, but it can hardly be demonstrated except in the later increase of efficiency.

2 The second reason for denying that poor motivation explains the poor learning of man, in his first visual experience, comes from the observations of Riesen (1947) His chimpanzees, reared in darkness, were certainly motivated both by hunger, and by therr strong drive to find and cling tughtly to an attendant, when they were out of theur living cages Yet there was no sign that either hunger or the desire to cling had taught them, in 40 to 50 hours' visual experience, how to discriminate the white-clad attendant from any other part of the environment. Astonishing as it was, the chimpanzees appeared to be completely avisual at this stage of the expenment

Moreover, in further tests, a strong electric shock falled in a dozen trials to set up any avordance whatever of a large, distnctive stimulus object. After a single tral, normal anumals of the same age and in sumilar crrcumstances showed violent avoidance of the object with such painful properties In the slowness of their first visual learning, man and chimpanzee are in the same class The human slowness is not due to defects of motivation, but points to some fundamental property of the learning process in primates.

REIATIONSHIP OF LEARNING TO PHYLOGENESTS The conclusion that the first learning differs radically from the later needs one most important qualification. the difference depends on phylogenetic level.

The evidence so far has been for the higher primates only. It was shown in an earlier chapter that traning in pattern 
vision is slower for the rat reared in darkness than for normal rats, but the dufference is not nearly as great as for chmpanzee or man The rat reared in darkness is capable of a selective visual discrimmation, definitely learned, after a total visual experience of less than 15 mmutes (Hebb, 1937a, pp 113-115). He requires six times as many trals as the normal to learn a discrummation of horizontal from vertical stripes, and twice as many for erect versus inverted trangles, but within an hour or so his behavior cannot be distingusshed from that of normal animals. As we have seen, the corresponding time for prunates is a matter of weeks or months

There are no comparable data for other species, but some insect behavior suggests strongly that the first learning of the invertebrate is still quicker, and much prompter in reaching full efficiency than the rat's What learning ability there is seems to appear full-blown, with little or no apprenticeship needed The bee for example on first emerging from a completely dark hive fles off and $z s$ able to find the entrance to the hive again. We know also that finding the hive depends on vision. The behavior indicates that the insect's learning starts out, from the very first, at the mature level of efficiency (Much that we attribute to mstnct, because no prolonged learning is evident, might thus be due to learning that needs only a few seconds for its completion. The associations that are formed may be only certain ones to which the nervous system is espectally adapted [Tmbergen, 1942, p 82] - heredity would still have an overmastering importance, but learning may nevertheless be essentual to some apparently instinctive acts)

As we go up the phylogenetic scale, then, we find in mature animals an mcreasing ability to learn complex relationships, but also, surprisingly, a slower and slower rate of learning in infancy.

This does not refer merely to the fact that higher animals have a longer period of physical maturation. We have always known that a rat grows up, and develops whatever capacines the adult rat has, in three months-or a dog in six months, whereas a chimpanzee takes ten years, and a man twenty years. We have thought, I suppose (If the question ever came up at all), that this longer period of behavioral development meant only that maturation takes longer in the primate, and that with 


\section{Development of the Learning Capacity}

less mstnct he has more to learn But the clnncal and expenmental evidence points to an additional factor. Given a really new and unfamiliar set of sensations to be associated with motor responses, selectively, the first definite and clearcut association appears sooner in rat than in man, and apparently sooner in the insect than in the rat.

We commonly regard quick learning as the main distunction of higher species, and in certain conditions this is true Normal man can glance once at a face and remember it for years. The chimpanzee Bimba was pricked once with a lancet and never again would permit it to be brought near her-but with no avoidance of other objects of the same size or roughly the same proportions." This is something completely outside the rat's scope. We think of it as intelligent learning, and are prone to regard it as an innate property of the primate brain It cannot be innate, however, as Ruesen's evidence shows So also with the abluty to remember faces Miner's (1905) patient, described as exceptionally intelligent despite her congenital cataract, two years after operation had learned to recognize only four or five ? faces and in daly conferences with two persons for a month did not learn to recognize them by vision The human baby takes six months, the chimpanzee four months, before making a clear distunction visually between friend and enemy Evidently, this is a period of learning as well as of maturation: not just a matter of waitng until certain neural structures are fully grown, with learning then at a typical adult rate.

There have been, in general, two schools of thought concerning the rate of learning. The configurationsts, stressing the importance of insight, have been inclined to hold that learning occurs as a single jump, an all-or-none affaur proceeding by discrete steps ("noncontmuty theory"), therr opponents, that

- Dr Glen Finch diary of Bimba, Yerkes Laboratones of Prnmate Biology, 1940 It is relevant here, in discussing the nature of learning in higher species, to add a reference also to the remarkable learning capacity of rhesus monkeys that Harlow (Psychol. Rev, 1949, 56, 51-65) has demonstrated in a long senes of experiments Harlow's whole argument, showng how the learning capacity may be changed out of all recognition by prolonged experience, is a powerful reinforcement of the position adopted in these pages 
learning is graded in amount, built up steadily by small increments, and typically independent of any special factor of insight ("contmuity theory")

But it is impossible to avoid the conclusion that both types of learning occur, and that one is charactenstic of the mature anmal, the other mannly of the infant. There is insightful, single-tral, all-or-none learning-in the mature anumal, but never in the infant of a higher species. There is a slow-increment learning in the infant, in which no trace of insight whatever can be found, and in the mature anumal also when he has been reared in darkness and is learning to use vision It is reasonable to suppose in general that, the less famliar the situation or the task to be performed, the more important slow-increment learning becomes But it seems also that few situations can be set up in which there is nothing familar, so that it would be very hard to find an mstance of learning in the mature anmal in which there is not some effect of insight.

We are now in a position to define the relationshyp of the learning capacity to phylogenetic level There is no evidence to support the idea that learning in general is faster in higher species-even at maturity In the infant, the evidence is conclusive that the rate of the first learning is slowest in the highest species, quite apart from slowness of maturation The distinctwe charactenstic of learning in higher species is the ability to handle complex relatonships, and handlé them as quackly as lower species can handle simpler ones Man can learn to unfasten a latch quicker than the chimpanzee, the chimpanzee quicker than a rat, but, if we take the learning at which each species is most efficient, there is no good evidence that one is faster than another

Lashley (1929b) has made this point effectively. After discussing an experiment by Pechstein in which rats and human subjects learned mazes of identical pattern, and in which the rats showed to rather good advantage-m one respect their scores were better than those of the human subjects-Lashley goes on to point out that with simple enough habits lower specres and the feebleminded learn about as fast as normal man. Such habits are not retarded, in rate of formation, by extensive brain damage There is also reason to think that immediate 


\section{Development of the Learning Capacity}

incidental memory occurs in lower species, and Lashley concludes "The comparative study of learning in different animals gives little evidence that evolution has brought any change in the rate of formation of the sumpler habits On the other hand, there is a faurly consistent rise in the limits of training and in the formation of complex habits with ascent in the phylogenetic scale."

In summary, then, the phylogenetic changes in the learning capacity are as follows (1) more complex relationships can be learned by higher species at maturity, (2) simple relationships are learned about as promptly by lower as by higher species, and (3) the first learning is slower in higher than in lower species.

CONCEPTUAL DENELOPMENT AS THE BASTS OF LEARNING Finally, before turning to the question of neural mechanisms, I want to bring together some of the behavioral evidence that throws light on how the learning capacity changes with growth. In general, it is a conceptual development, rather than the elaboration of a number of specific motor responses. Perceptual organization is also involved, but percept and concept are intimately related, and the term "conceptual development" will do to cover both.

The best smgle illustration of how one set of expenences can facilitate the formation of a new habit, mvolving a new stumulus and a new response, is one that has already been given: in learning to distinguish chimpanzee faces and remember them (or of course the old example of the westerner's difficulty in recognizing a partcular Chinese face, before he has seen many Chinese) Learning to name Pan, Jack, Frank, Don, and so on, makes one later able to name a new chimpanzee, Balt, much more quickly. The exposure to a number of individuals sets up some sort of conceptual type, from which individual deviations become very noticeable.

This sort of facilitation in learning seems quite general, partcularly in what can be called intelligent (as distunct from rote) learning, with meaningful material That such a facilitation affects intelligence-test scores was the only interpretation that seemed possible of certain aspects of behavior following brain injury, or in old age (Hebb, 1942a) In a later chapter I shall present evidence showng that the behavior of the blind 


\section{Early and Later Learning}

rat is permanently changed by earher visual experiences. Jackson (1942) and Burch (1945) have independently shown an influence of expernence on the insight of young chmpanzees Burch has made it clear that this does not reduce insight to rote learning (thus Kohler's classification of behavior retains its value), and yet insight depends on the earlier experience.

How then does the earher experience work? In the very first stages, to judge from Ruesen's experument and the maternal brought together by Senden, it operates to establish the perceptual elements discussed in Chapter 5 These are the entities that make up more complex perceptions Organizing such elements in the vanous sense modes would lay the foundation of all later responses to the environment. Secondly, there is a period of establishing simple associations, and with them conceptual sequences-the period in which meaning first begins to appear Finally, the learming charactenstic of the mature anmal makes its appearance

This later learning is essentially conceptual Even in the rat, maze learning requires the notion of the stimulus as acting to arouse conceptual activities, which in turn control motor activity (Lashley, 1944, Tryon, 1939) In man the conceptual actuvity has an even greater and more obvious role. There have been a number of attempts in psychology to treat language theoretcally as a collection of conditioned reflexes, specific stimulations directly controlling specific responses. But consider such pecularties of human learning as the following.

Certain features of the development of language in small children are very instructive. With opposites such as up-down, back-front (of a house), en-out, or left-right, a very interesting confusion can be observed in some children at about the age of two to four years. (Some doubt about left and night, of course, is often seen in adults, while other opposites, such as hot-cold, or black-white, never seem to give any particular trouble.) The confusion of $u p$ is only with its opposite and never with "in" or "back" This means that the word has first acquired an assocration with a definite, limited set of conceptual coordinates. The confusion, when it is observed, may last for months. In this period the word up has its prompt, clearcut association with the vertical dimension, but no association with a particular 


\section{Development of the Learning Capacity}

motor act such as raising the hands or looking upward at sky or celling With such facts, it becomes nonsense to explain man's conceptual development as exclusively consisting of verbal associations.

The relationship of (a) stimulus, (b) central activity or concept, and (c) motor response is clearest in the notorious diffculty of choosing between left and right, to bo observed by anyone who tries to teach twelve-year-old children to "nght turn" promptly on command. Here we have a definite auditory stumulus, the word right, and a definite motor act to be assocrated whth it. One might expect the assoctation to be immediate, or at the very least to be set up as a conditioned reflex in a few trals-the more so since the child has had some such training smce infancy. But we know that this does not happen; the discrimination is certanly much harder to make than that of up and down, and this in turn harder than black and white The child can very readily learn at the age of three that "nght" and "left" each refers to a side of the body-but ah me, which one?

Here, in the discrimination of right and left, is the real paradigm of adult learning What is set up first is a conceptual organization. By the age of sux the word "nght" clearly and immediately means sidedness to the child. A considerable conceptual elaboration has already occurred, and the stumulus effec. tively arouses that structure, but it arouses no prompt, specific response The response may come later, but for long it remains vacillating and unpredictable.

Consider again the role of analogy in human thought, and the figures of speech that betray it even in scientufic work. The pons and island and aqueduct of cerebral anatomy, the wave of sound and cycle of sunspots in physics and meteorology; the rwe of the blood-sugar level, and the limen of stmulation in physiology-all these are as enlightening, concerning the nature of learning and intelligence, as the child's confusion of left and right Such figures of speech are at the very least an and to memory, even when it has a totally new reference, the famuliar term is more easily recalled than a neologism Using it is therefore more than an economy of language The underlying 
analogy with something known already is an economy of thought as well, an economy of effort in learning and understanding.

This fact is at the least surprising, since exactness of meaning is essential to scientific thought, and since the laws of leaming as we know them would suggest that there must be a considerable interference between the old meaning and the new one given to a familiar term. That is, there should be interference unless terminology corresponds to thought and the new concept is only a modification of the old.

The worker in the laboratory does not merely report and expound by the and of analogy, that is how he thinks, also The atom was once a hard little round partcle, or later one with hooks on it. Recently it was a solar system. The classical dispute of physics about the nature of light was really asking, Is light like a shower of pebbles, or like ripples in a bathtub? The ultumate answer, Both, was one that was hard to accept Why? Because it fitted into no pre-exasting conceptions, waves are waves, and pebbles are pebbles-there is nothing in common experience that has the properties of both We know well that screntific thought travels by short steps, the individual thinker never gets much in advance of his fellows, and his ideas are born of existing ideas. A new conception, like Woodworth's "schema with correction," must be mainly composed of earlier ones New learning is faclitated by old

But, as the history of the theory of light suggests, it may be limited or canalized as well I have used the analogy of an apprenticeship, in visual learning. Now an apprenticeship in one trade may help in another, but not as much as the one drrectly studied. It is not just any learning that faciltates any other learning at maturity

Nissen, Machover, and Kinder (1935), for example, have shown how the visual learning of chldhood may be selective in its later effects West African natives made low scores on form boards, in an intelligence test In this sort of test the subject is asked to fit a senes of wooden blocks with simple geometrical shapes into holes with the same shapes, as fast as he can In conversation, Dr Nissen has made the point that the low scores were not due to slowness of movement, but to a slowness in identifying shapes-a slowness of perception. 
At the same time, he found himself just as inferior in seeing things in the bush that seemed completely obvous to the natove. Suppose now that a native and a city-dwelling scientust were shown the trail of a new animal in the same habitat. though it is strange to both, which would remember it better, and be able to recognize it on a second occasion? What a natve could learn from a text on geometry, or what one of us could learn about following a tral, must be far more a function of pre-existent learning than of the inherited properties of our respective brains

It is of course a commonplace that the experiences of childhood have a permanent effect on one's attitudes, interests, and even ablities Lorenz (cited by Tunbergen, 1942) has demonstrated the effect experimentally in burds With mammals, everyone knows that taming is easiest in mfancy, with a lasting effect The gun-shy dog, conversely, is an example of a lasting falure of emotional adaptation though the disturbance need not be set up in infancy, it does demonstrate an intractable effect of a first experience. Hunt (1941) has shown an effect of early hunger on later hoarding by the rat. As to abilities or intelligence, it is now generally accepted that races and peoples cannot be compared in hereditary endowment, since low scores may be due to cultural background That the level of problemsolving at maturity, then, may be permanently influenced by childhood experience is an accepted psychological principle, despite a certain inconsistency of the theorists who think that the Negro's low IQ is to be explained so, but seem to have forgotten that the poor white's may be in the same class

To this point we shall return, in discussing the nature of intelligence Here I have attempted to define the general relatonship of first learning to later learning, in terms of the behavioral evidence Let us now tum to the physiological processes that must explain the relationship as well as the peculıari-tes of primary or aborigmal learning

\section{THE STAGE OF PRIMARY LEARNING}

Among others, the preceding discussion has raised the problem of explaining the greater inefficiency of early learning in higher 
species. To deal with it, we can return to the schema of perception and reparr a deliberate omission The schema made almost no mentron of the spontaneous firng of the association areas of the cerebral cortex. Taking this into account, a queston is rased that has really wide signuficance For the moment, it can be put in this form In what crrcumstances can one suppose that a stumulus will have the same central effects on two different occasions, so that cumulative learning is possible?

It has already been said that an afferent excitation does not arouse mactive tissue but feeds into an activity that is already going on A constant net result, when a stumulus is repeated, requires some constancy in the spontaneous activity in the association areas upon which the afferent activity impinges. The perceptual learning that has been schematized in the preceding chapters depends on some consistent central action of a repeated stumulus. We need not assume a constant pattern of the background activity, at all tmes, to satisfy the schema, but it does demand at least a frequent recurrence of the same pattern. This is necessary if the stimulus is to have a cumulative action and build up the "assembly" of cells that constitutes the first learning.

In the answer to this problem hes an explanation of the slower first learning in higher species and, indurectly, as we shall see in later chapters, an understanding of some of the problems of motivation and emotion in the half-grown or full-grown animal.

There seem to be two main factors that would make for some consistency in the activity of the association areas at different times One can be referred to as an intrnsic organization, in that activity, the other is the steadily increasing influence of the infant's environment.

The intrusic organtzation of cortical activity is so called because it is opposed to the organization imposed on the cortex by sensory events. It appears at burth ${ }^{*}$ in the large slow waves

- I 0 , it is "innate", but it may or may not be unlearned. There are two possibilities (1) that there are pacemakers which are inherently such as to dominate other neural cells and produce the synchronous fing, and (2) that the synchrony is "learned"-established in utero as a result of the newral activity itself The comparatively slow change of the infant's EEG toward the adult pattern, and the lack of any sharp discontinuty between the two extremes, suggest the second interpretation Supposing that the 
of the infant's electroencephalogram, and in the sleep or coma of older subjects. At burth, that is, the intrinsic organization is completely dominant, in psychological development its domlnance decreases, but it contnues to recur periodically, generally in a drurnal rhythm. Its behavioral correlate is a heightened limen for sensory stimulation and a marked absence of the persistently drected activity that we call voluntary and purposeful.

This does not mean that there is any sharp line that divides infant behavior, or sleep, from the behavior of the wakeful adult, it does not mean, ether, a hard-and-fast distnction between the corresponding EEG's But there appear to be two extremes in the organization of cortical activity that correspond exactly with the two extremes of learning ablity discussed in the last secton. One is establsshed early, interrupted rather than supported by a varied sensory activity, once the alternate organization begins to appear, manifested by large potentials in the EEG, and correlated with an inactivity of the musculature or an amless, undrected activity and a slow and mefficient learning

firing of central cells is onginally at random, the synaptc changes discussed earlier would lead to an increased coordination of fining and the establish. ment of massive self-exciting systems These might be comparatuvely simple closed curcurts at first but would tend to steadily recrunt other cells and later other curcunts This is the "growth" referred to in the discussion of perceptual development (p 76) Without the constant sensory disturbances resulting from the motor activity of the walang animal, the growth would make for local integration, and hypersynchrony, with them, the integration is in anatomically diffuse systems and reduces local synchronizatoon

This discussion makes a good deal of the electroencephalographic endence as the most direct index of physlological organization in the nomal living bram It is wse to recognize explicitly, however, that the inder is a very rough one $I$ have treated the slow waves of the infant's EEG, and those of adult sleep and coma, as having the same meaning Apparently they may have, in one respect as indicating a local synchrony of finng in cortical neurons (see the assumption made earlier, $p$ 8) But in other respects there may be significant differences Indeed, there must be, and future developments in electrical recording may reveal them For the present, the important thing is to see that there is in one respect an idenity of cortical activity in infancy, sleep, and pathological conditions which is at the opposite extreme from that of the normal walang adult, as seen both in the electrical record and in the presence or absence of sustained purposeful behavior 
The other is slowly acquired, dependent on a constant change of the sensory influx for its maintenance for any period of time, produces a flattened, irregular EEG, and is the precondition of the normal adult wakng behavior and the adult level of learning

Note that what is discussed here is a variation of organized activity in the cortex In the later discussion of fear and neurosis the notion of a disorganization will appear, producing neither quietude nor purposeful activity but incoordination and autonomic activity Intrinsic or primitive organization, and disorganization, are to be distangusshed

Against the background of the intrinsic organization, the infant's first visual learning could proceed steadily, although slowly, wrth no disruption If the first sensory excitation feeds into an organized activity, where cells are firmg rhythmically in large populations (which I assume is what the large waves of the infant's EEG mean), the argument of the schema can still stand, although visual learning must be slower than if the cells of area 18 were always ready to be fired, and never refractory, when an impulse arrives from 17 The only change in the schema is to show still another reason for the slowness of first learning in the higher primates.

At the same tume, an explanation of the difference from lower species appears The process of perceptual learning must be thought of as establishing a control of association-area activity by sensory events The larger the association areas, both absolutely and relative to the size of the sensory projection areas, the slower the establishment of such a control must be and the less nigid and more complex its final form.

Let us suppose, as we safely may, that the principles of central organization in other sense modes do not differ from those of vision. Anatomical differences in the various sensory systems may affect the complexity of discrimination, fineness or acuity of discrimination, and so on They may lead to a greater relative importance of temporal integration $m$ one sense and of spatial in another, as in hearing and sight But these anatomical differences do not imply different principles of central neural integration in sight and hearing, or in touch and smell

Our problem essentrally is to see how a particular sensory event can have the same central effects on different occasions 


\section{Development of the Learning Capacity}

despite spontaneous central activity If we conclude with Adrian (1934) and Weiss (1941a) that the unstumulated neural cell must eventually fire spontaneously, we must also accept Adrian's conclusion that this cannot happen in the cell that is exposed to persistent bombardment. As long as the receptor surfaces are being stimulated, therefore, the sensory projection areas of the cortex must remain completely and constantly under environmental control

Also under control are the fibers that lead from the sensory areas into the association areas According to the schema of perception, this control is extended gradually, synapse by synapse. Considering the association areas as made up of a population of transmission units, two factors must affect the length of time needed to bring all these units under control One is the number of controlling fibers leading from sensory areas into association areas The second is the number of transmission units in the association areas themselves.

With cortex of a given size, these two factors may be considered to be roughly proportional to the size of the total sensory cortex, and the total association cortex. It then follows that the length of the primary learning period will be roughly proportional to the ratio

$$
\frac{\text { total association cortex }}{\text { total sensory cortex }}
$$

which can be called the A/S ratio The sensory projection areas are directly under environmental control, and if they are large, with respect to the association areas, and so project a large number of fibers into the association cortex, their control should be quickly estabhshed If the sensory projection is small, association cortex large, the control will take longer, the perod of "primary learning," that is, will be long.

But we may have to consider a further factor. With the same A/S ratio, but with different absolute size of bram and a larger absolute number of transmission units in the association areas, it seems that the larger brain might also make for slower first learning A larger number of transmussion umits means a greater variability in the spontaneous activity of the association areas, as well as a larger number of synaphe junctions over which 
sensory control must be extended It will be recalled that the synapses in question are not the minumum number through which an afferent excitation nught conceivably reach the motor cortex. We are dealing with recurrent crrcuits and complex closed systems. An absolutely greater number of transmission units in the association areas would probably make for greater varrability in early spontaneous activity and a greater ultmate complexaty of organzzation. Both umply that a sensory control might be established more slowly in a larger brain, even with the A/S ratio constant.

The sensory projection areas are whdely separated in the bram, almost as if to maximze their influence Only in the frontal pole in primates (and to a less extent in the temporal pole) is there cortical tassue that is far removed from a sensory projectuon area. We can then regard the stage of primary learning as the period of establishing a first environmental control pver the association areas, and so, indrectly, over behavior. With this propostion we have at once an explanation of the slowness of the first learning in prumates, in which bram and A/S ratio are larger, and of the ultumate level of the prumate learning capacity.

The numencal aspect of this fraction cannot be stressed, since presumably there are other factors of finer neural anatomy, of metabolssm, and so on, to be taken into account It may bo suggested, however, that the size of association cortex, and the A/S ratio, when these can be expressed in terms of cell counts, may provide the basis of a better morphological index of the level of behavsor than the brann-weight/body-weight rato. This of course is not testable at present. No cell counts are avalable, and psychology bas so far found few satsfactory compansons of intelligence as between different species. We have not even any exact data on the gross size of the sensary cortex in different species.

But, for widely differing phylogenetc levels, a hrerarchy of "intelligence" (or psychological complexity McBnde and Hebb, 1948) can be assumed, which corresponds to gross defferences (1) in size of the cerebrum, or (2) in the proportion of afferent to internuncial neural tassues. In the lower vertebrates, the cerebrum is small, and the afferent systems are massive in comparison with the internuncial. Withn the mammalian senes, 


\section{Development of the Learning Capacity}

there are differences both of absolute and relative suze of assocration cortex which may be assumed (in the total absence of any exact data) to have a relevance to the greater speed with which the "lower" species can learn to respond selectively to the environment, and to the comparative simplicity of the behavior when it is fully developed.

These anatomical considerations draw attention to another point. The learning ability of higher species at maturity is not merely the capacity for a greater number of associations or for associations that involve finer sensory discriminations. The behavior also shows a less durect control by the stumulus of the moment, from the immediate environment In larger association areas the central phase sequence can be more complex it must still be organized and ultimately controlled by the relatively smaller sensory projection areas, but the phase sequence can escape the direct control more frequently and for longer periods The possession of large association areas is an explanation both of the astonishing inefficiency of man's first learning, as far as immediate results are concerned, and his equally astonishing efficiency at maturity.

\section{MECHANISM OF ADULT LEARNING}

The question has been rased as to how, if the association areas of the bram have a contunuous and variable activity, a repeated stimulus can have the same central effect on separate occasions, to make cumulative learning possible The question was answered for the infant, and the answer helped to account for the slow first learning of higher species It can now be answered for the adult

Fortunately, it is no longer necessary to account for a cumulative effect of stimulation that is superimposed on an independent activity of the association areas (fortunately, because this activity must be extremely vanable in the waking adult) The learning occurs when the events to be associated can already command organized trains of cortical activity, in other words, when the environment has a control of the association areas that can be repeated, so that the central activity is not at 


\section{Development of the Learning Capacity}

there are differences both of absolute and relative suze of assocration cortex which may be assumed (in the total absence of any exact data) to have a relevance to the greater speed with which the "lower" species can learn to respond selectively to the environment, and to the comparative simplicity of the behavior when it is fully developed.

These anatomical considerations draw attention to another point. The learning ability of higher species at maturity is not merely the capacity for a greater number of associations or for associations that involve finer sensory discriminations. The behavior also shows a less durect control by the stumulus of the moment, from the immediate environment In larger association areas the central phase sequence can be more complex it must still be organized and ultimately controlled by the relatively smaller sensory projection areas, but the phase sequence can escape the direct control more frequently and for longer periods The possession of large association areas is an explanation both of the astonishing inefficiency of man's first learning, as far as immediate results are concerned, and his equally astonishing efficiency at maturity.

\section{MECHANISM OF ADULT LEARNING}

The question has been rased as to how, if the association areas of the bram have a contunuous and variable activity, a repeated stimulus can have the same central effect on separate occasions, to make cumulative learning possible The question was answered for the infant, and the answer helped to account for the slow first learning of higher species It can now be answered for the adult

Fortunately, it is no longer necessary to account for a cumulative effect of stimulation that is superimposed on an independent activity of the association areas (fortunately, because this activity must be extremely vanable in the waking adult) The learning occurs when the events to be associated can already command organized trains of cortical activity, in other words, when the environment has a control of the association areas that can be repeated, so that the central activity is not at 
random and the stumulation can impinge on the same central pattern when the training situation is repeated.

I have made it an essentzal condition of learning that two central events occur together. The reader is reminded, however, that this is not always a sufficient condition In discussing the question earler ( $p$ 77), I considered the probability that two systems would acquire an interfaciltation by being active at the same time The systems referred to, at that point in the development of the theory, were newly organized ones, and the question was of the first association of one system with another during primary learning The conclusion was that the ease of association must vary greatly, even when the two systems lie intertangled in the same gross region of the brain They might promptly coalesce, at one extreme, or might, at the other, fail to do so despite often being active at the same time, over a long penod It was concluded also that the larger the system the greater the probability of its establishing an effective interfacilitation with another-provided that the two are well organized, so that arousing part of one will arouse the whole

But we are now interested in the associations formed at maturity, between much more complex processes Learning at maturity concerns patterns and events whose parts at least are familiar and which already have a number of other associations. This changes the problem considerably It means that the learning is not an association between totally unrelated processes. It must concern a complex of cell-assemblies, and elaborato phase cycles (in the jargon of my schema), and amounts to a strengthenung of faciltations, not a setting up of new connections between wholly unrelated activities

The characteristic adult learning (outside of psychological laboratories) is learning that takes place in a few trials, or in one only It seems always to involve a recombination of familiar perceptions and familiar patterns of movement Thus one can typically remember a new name when given name and surname are already famuliar, or a new face when it is one of a racial group to which one has long been exposed. Vary these conditions, and repeated effort may not do the trick. Complicate the problem even by requining that the new name and new face be learned simultaneously, and the difficulty is increased, it is 


\section{Development of the Learning Capacity}

usually overcome only by repeating the name and looking at the face a number of tumes in a short interval So adult learning is typically an interaction of two or perhaps three organized activities, being organized, they are capable of a contunued existence after cessation of the stimulation that set them off, which gives time for the structural changes of permanent learning to take place For the theory being developed, a prompt learning is possble when the stimulation sets off well-organtzed phase sequences, but not otherwise. This organzed activity of the association areas is subject to environmental control To the extent that the control is effective, and re-establishes the same central pattern of activity on successive trials, cumulative learning is possible.

Adult learning is thus a changed relationship between the central effects of separate stumulations, and does not directly concern the precipitating stimulus or, primarily, the motor response whose control is imbedded in the central actinity

The same conclusion is reached from another approach. Experiments on learning have shown agam and again that the nonsense syllables tob, del, rec, $t l l$, and so on, are harder to memorize than the more complicated items tobacco, delights, recommend, and heartily, and still harder vthan the sentence “I recommend the delights of tobacco heartly" The event with meaning is best remembered, moreover, it is the meaning that is remembered rather than the specific strmulation that aroused meaning (McGeoch, 1942) That is, the central effects of sensation are what enter into an association, rather than the comparatively simple sensory event itself This seems especially true of the most efficient learning-the kind that is established most easily and persists longest.

Such learning may be diagrammed as in figure 14, where each curcle represents a conceptual activity The concept of an object or place is an irregular cycle, each phase of which is the, activity of a cerebral cell-assembly If a large enough part of this phase cycle is aroused, the whole becomes actrve. Thus in figure 14 the concept $A$ was originally organized by an interaction of hearing, touch, and vision Once orgenized, it may be aroused by hearing alone, or perhaps by hearing and touch, but the essental association between $A$ and $B$, resulting from simul- 
taneous activity, would be the same whether each was aroused by vision, or whether one was aroused by hearing and the other by touch.

In this way learning may be "free" of any particular sensory cues (Tryon, 1939) The rat may learn a maze by the and of cues from vision, smell, touch, and kmesthesis; the maze once

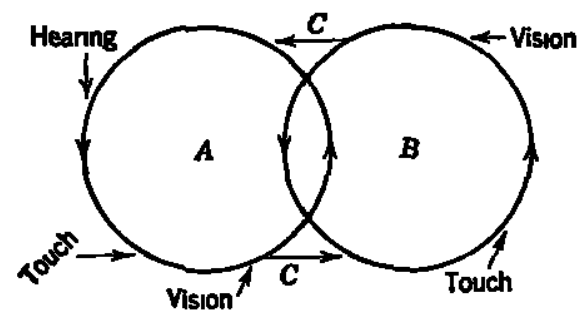

Freung $14 A$ and $B$ represent two conceptual activities $C, C$, possible connections between $A$ and B These are not smple closed neural crreuts, nor even the more complex "assembles," but "phase cycles" systems of assembles whose several activites are temporally integrated and tend to recur in an irregular cycle $A$ was ongmally organized by auditory, tactual, and vasual stmulation (that 1s, it involves assemblies in each of these modes), $B$, by visual and tactual stmulation When these cycles are well organized, therr acturity may be inithated by part of the ongunal stmulaton- $A$ for example by heanng, touch, or vision When $A$ and $B$ are simultaneously active, they may acqure an interfaclitation which is diagrammatically represented by $\boldsymbol{C}$ and $\boldsymbol{C}$. The learring is independent of any partcular strmulation, the association might be set up by two visual stmulations, but be manfest later when $A$ is aroused by hearng, or $B$ by touch

having been learned, any of these cues may be disturbed or removed, but the learning persists and utilizes those that remain We shall see in a later chapter that the behavior of the blund rat is permanently affected by his having had vision during the penod of development. The rat blinded at maturity solves certain problems that rats blinded at birth do not The properties of the concept $A$ (figure 14) depend originally on vision, but a rat might be blinded, after $A$ had been developed, and still retain the concept as one that can be aroused by heanng and touch alone.

Now as to the mode of association between two such concepts The two neural structures may have enough chance ana- 


\section{Development of the Learning Capacity}

tomical convergences (Chapter 4 ) that a durect interfacilitation is established. This is represented (still quite diagrammatically) by $C, C$ in flgure 14 Then, even though the stumulation arousing $A$ and $B$ is brief, they may contmue active long enough for synaptic knobs to develop The result would be one-trial learning, mvolving two separate neural systems

But this, it seems, is unllkely When new connections are to

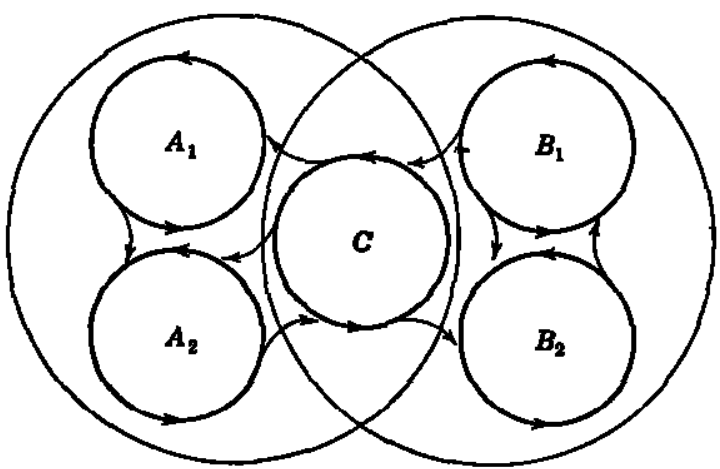

Figure 15 To illustrate the possibility that a subsystem, $C$, may act as a link between two systems (conceptual complexes) One concept is represented by $A_{1}, A_{2}$, and $C$, the second by $B_{1}, B_{2}$, and $C$ The two systems have a subsystem, $C$, in common, to provida a basis of prompt association

be set up, as in figure 14, it is probable that a number of tnals would be necessary, and the diagram is best considered as representing rote learning The facts already discussed have indcated that one-trial learning occurs only as the association of concepts with "meaning"-havng, that is, a large number of associations with other concepts. Figure 15 diagrams another possibility, which can be given more weight A perceived object consists of a number of perceptual elements (p 83) The same elements recur in different perceptions, so that two concepts to be associated may have phases (assembly actions) in common. These would be ready-made links, needing only to be strengthened to establish the association

But more the perception of an actual object (that can be seen from more than one aspect, and touched, heard, smelled, and tasted) mvolves more than one phase cycle It must be a hier- 


\section{Mechanism of Adult Learning}

archy: of phases, phase cycles, and a cycle or senes of cycles. ("Cycle" is-of course temporal: referring not to a closed anatomical pathway but to the tendency of a senes of activities to recur, irregularly ) The two ideas or concepts to be associated

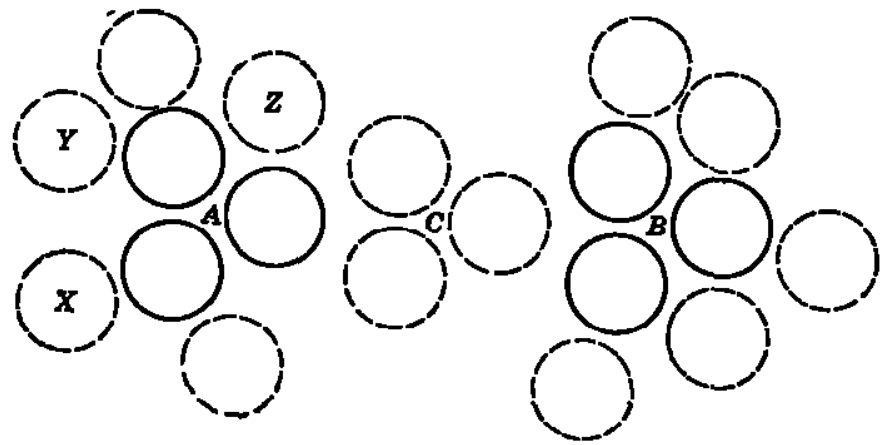

Ficunx 16 Diagramming in another form the same principle as in figure 15 The complexes $A$ and $B$ are sensorily aroused at the same time Solnd circles represent systems that are reliably aroused, broken crrcles, ones that are facilitated by the activity of adjacent systems, but not reliably aroused by them That is, the "froge" systems $X, Y, Z, C$, and so on, recenve a facilitation from $A$ which is often not sufficient to arouse them, which ones are active will depend on preceding activity in the thssue and accidents of concomitant stimulation The complex $C$ might or might not be aroused as a sequel to $A$, or to $B$, but when both are active simultaneously, $C$ is more lukely to be aroused. Thus the subject associates an object $B$ with the object $A$, because both are associated with something else, $C$. a familhar trick in memorizing lists of words or nonsense syllables in learning experiments But the processes $A, B$, and C all occur within what is grossly the same tussue, not spaced as in the dragram, and when by virtue of $C$ 's activity $A$ and $B$ are persistently active together, they may slowly buld up a direct interfacilitation-so that $C$ can drop out eventually, leaving $A$ linked to $B$ directly, by a short-curcuitung. (Woodworth, 1938, p 34, McGeoch, 1942, p. 166 )

might have, not only phases, but one or more subsysterns in common. This is suggested by the diagram of figure 15 It should provide an even more effective lonk Such a mode of association is possible only between complex systems, and it may be recalled again that a complexity of meaning is more readily remembered than a simpler perception without meaning.

The same general principle is involved in figure 16. This 
represents the famllar fact that a subject required to learn pairs of words with no obvious relationship will invent relationships, and will himself supply extra terms in doing so. The subject trying to associate conceal with above may do so by thinking above = ceming-conceal, or with marble-punish, may think of being punished Fon PLAXING marbles Fon kmaps (Woodworth, 1938). The subject is making his task more complex when he chooses to remember three or four terms unstead of two, but he also makes it easier. Thus figure 16 shows how two concepts $A$ and $B$ may be associated if a third one $(C)$ is aroused to which $A$ and $B$ are already related

The prompt learning of maturity is not an establishing of new connections but a selective reinforcement of connections already capable of functioning Observe, however, that this account differs from traditional association theory in at least one respect, which is of the greatest importance for applymg the theory to the results of experıment Two concepts may acquire a latent "association" without ever having occurred together in the subject's past experience. Although the "association by similarity" of older theory recognized this fact, the explanation seemed to depend on the idea of identical sensory elements The present theory suggests that the identical elements may be conceptual rather than sensory, that is, two things may seem similar without exciting the same receptors.

The concept $A$ in figure 16 is capable of arousing, or facilltates, $C$, but the facilitation may be from one of the subsystems of $A$ on a subsystem of $C$, so that $A$ and $C$ need never have been active together at any earlier time. Or, as in figure 15, two systems may coalesce promptly because of having a subsystem in common. It seems likely that something of this sort is the explanation of the happy figure of speech, which links two sets of ideas not previously associated the more unlke they are, superficially, the more effective the figure, and yet it is effective only because something about the structure of the two ideas is the same (For examples of such pairs of ideas, the reader may consult the discussion of scientific analogres earlier in this chapter )

My treatment of learning here is tailored carefully to the experimental facts, but it also follows naturally from the original 


\section{Mechanism of Adult Learning}

neural schema. At this point, in the emphasis on pre-existent associations in learning, we have come to a classical problem that we can examine a little more closely. the meaning of "meaning"

The implication of the preceding paragraphs is that a concept is not unitary Its content may vary from one tume to another, except for a central core whose activity may dominate in arousing the system as a whole To this dommant core, in man, a verbal tag can be attached, but the tag is not essential. "The concept can function without 1 , and when there is a tag it may be only part of the "fringe" sometimes aroused with the domlnant subsystem, sometrmes not The conceptual activity that can be aroused with a limited stumulation must have its organized core, but it may also have a fringe content, or meaning, that varies with the crrcumstances of arousal

Thus in figure 16 the activity of $A$ may be accompanied by the activity of $X$ on one occasion, or of $Y$ and $Z$ on another Which will happen is not indeterminate, but depends on the excitability of each subsystem at the moment, and on the faciltation from other concurrent sensory and central activities.

Now consider the subject learning a list of words again Followng one train of thought, in which an activity occurred which faciltates activity in $C$ (figure 16), the occurrence of $A$ and $B$ (also facilitating $C$ ) leads at once to the sequence $A-C-B$ On another occasion, $A$ and $B$ at first might have excited no common system In such crrcumstances, the subject would look at the words, "think" about them (that is, $A$ and $B$ are persistently active, with a varying fringe activity), until the faclitation on $C$

- It seems likely that there is a great deal of conceptual acturty that is unreportable (and so "unconscious") in human thought above all, in what can be called inturtive judgments (Hebb, 1946a) Despite reliable and predictable verbal responses in the recognition of emotion, for example, a subject may be quite incapable of saying what considerations determined his choice of terms There may, consequently, be concepts in man that do not have a verbel element, just as there are in animals On the other hand, it seems quite clear that many concepts are fundamentally and essentally verbal-the "core" is a word or other symbol, without which the concept could no longer be an element in thought (function as a neural system) If we recognize, first, that there are nonverbal concepts, we must recognize secondly that some concepts are verbal only 


\section{Development of the Learning Capacity}

from some one of the fringe activities suddenly becomes liminal, and the relationship $A-C-B$ is "perceived"

Evidently such changes are what has been called, in the psychological literature, a "restructuring" of thought, and the sudden activation of an effective link between two concepts or percepts, at first unrelated, is a simple case of "insight" (p 158) This is a topic that will be discussed more fully in the following chapter The mechanism of the sudden perception of new relationships is, I propose, the one that has just been discussed, in its simplest form.

This account does not, however, sufficiently recognize the orderly, drected, persistent feature of the phase sequence that leads to insight. a problem that presents major difficulty (Humphrey, 1940), and is considered in the following chapters in the treatment of attrtude, motivation, and pleasure For the present theory, insight is a "chance" combination of faclitations from different phase sequences; but this is chance only in a limited sense, and given phase sequences that persistently return to a certain conceptual activity (motivation to solve a problem) the ultimate occurrence of insight may be quite predictable. What may not be predictable is just when the occurrence will take place, and this of course agrees with the facts of behavior.

\section{MAZE LEARNING}

Having considered how a certain land of human learning might occur, in which the subject given one word must reproduce a second, let us next see how the theory of phase sequences and "concepts" can be applied to the learning of a rat in a maze

Suppose that we have a simple maze with a starting point $A$, three choice points $B, C$, and $D$, and a goal $E$ As the rat runs the maze correctly, the envirónmental or sensory sequence would be $A, B, C, D, E$ The phase sequence determining such a run cannot be so simple, because of the central facilitation that may arouse a conceptual activity in advance of its sensory arousal ("expectancy"), and equally may arouse the concept of'a point that has already been passed ("recollection"). This can be diagrammed as

$$
A-e-b-B-a-c-C-\cdots E
$$


where the small letters represent centrally aroused complexes (each corresponding to the recognition of a point in the maze), and capital letters represent sensornly aroused ones, or actual perceptions

The existence of the procurrent items in the sequence above is both an inference from the neural schema and a reasonable mference from the literature on expectancy The recurrent item ( $a$ following $B$ ) is manly an inference from the schema, but it is suggested also by the backtrackng done by sophistcated rats in a simple maze

In a long and complex maze, with unsophisticated rats, retracing is usually thought to be simply an error, part of the rat's effort to reach food as quickly as possible But in the method of testing intelligence described by Hebb and Wlllams (1946), the rat often acts in a way that suggests something different. After reaching the food box the anmal may turn and explore a blind alley, or retrace the entrre maze (sometimes without a single error) His return to the food box is usually at a higher speed than the first part of the run, and he eats enthusiasticallyon the second tume of reaching food Behavior is determined by the central phase sequence, and this means that the sequence is recurrent as well as anticipatory. Sumlarly, "mental backtracking" is reported by the blindfold human subject learning a stylus maze (Woodworth, 1938, p 143)

Also, when one is finding one's way through an unknown city, or by compass through the deep woods or at sea, one has a contunual awareness of the supposed drection (1) of the goal and (2) of the earlier route, though the apparent direction of either may be quite wrong There are indications in both animal and human behavior that learning depends on setting up a unification of the total situation even when it cannot be surveyed as a whole It is not necessary for the learning that an accurate picture of the situation be achieved (Brown, 1932), only that the goal-concept dominate the phase sequence, and that each particular complex in the sequence corresponding to a correct run reliably evoke the next complex, with the resultant recognition of the durection of the next choice point

As an illustration when driving to the neighboring city, 18 miles away by a winding route (on a modern highway, that is, 


\section{Development of the Learning Capacity}

on which all the turns are very gradual), I persistently feel that the part of the highway on which I am at the moment leads nearly straight toward my objectuve. When a bend in the road approaches, the apparent drection of the city changes, each turn is remembered, inconsistently, as a turn toward the goal, except when two turnspre close together I am unable to list all the different segments of the route from memory, in the nght order, all I can do reliably is make the right turns at the right time

This proves nothing, perhaps, except that I am easily confused, but it ilustrates the point that a series of correct responses can be organized into a smooth sequence under the influence of a goal anticipation without requiring that the subject (whether rat or man) have any accurate or detaled image of the total situation The phase sequence determining such behavior need not be so well organzed that it can run off complete without sensory support. As I start driving, it consists of little more than an awareness of the starting point, the goal in a certain direction, and the next distunctive point in the route.

How is the sequence established? It will be clear, from the facts reviewed in the early part of this chapter, that the halfgrown or adult animal comes to his task with an elaborate conceptual organzation ready made, and that the learning is a modification of this to fit the particular propertes of the new situation Before one can attempt anything like an explanation of adult learning, therefore, it will be necessary to find out much more about the ontogenetics of behavior. The expenments reported in the following chapters hardly amount to a beginning at this task, until they are carned much further a discussion of the detals of maze learning must be speculation only The experuments referred to suggest, for example, that much of a rat's learning to run a maze is done in his home cage, before he ever enters the maze at all What I propose to do here and in the following chapters is outline the approach that is implied by the theory and define the problems that it rases for research

One of these problems is the development and control of hunger. Experumental evidence presented later (p. 193) ind1cates that there is an important element of learnmg in hunger. 
It is not always enough to have an animal lack food in order to get him to eat At the same time, the analysis of the control of eatung made by Morgan (1943) shows that no simple learning formula will provide for the known facts This problem, then, must be left to one side for the present Assume here that the foundations are la1d. the rat put into the apparatus will eat food when he finds it, will move about untl he does find it, and has

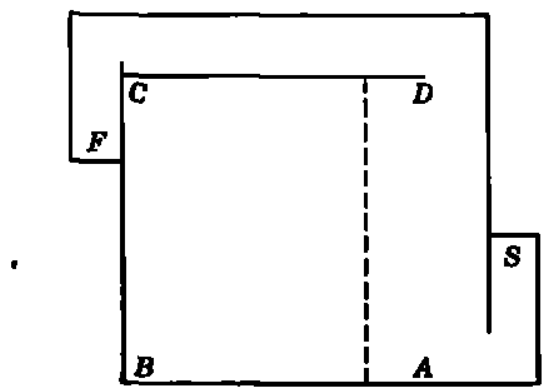

Figure 17 A simple maze problem, the simplest of those used in the method of testing rat intelligence, that is briefly described in Chapter 11 $S$, starting box, $F$, food, $A, B, C, D$, points in the maze The line $C-D$ represents the single barner used in this test problem, in the preceding problem, four barriers, dufferently placed, presented the rat with a more difficult problem. Crossing the broken line constututes an "error"

become used in prelimmary training to the general situation and has organized his perception of room cues, the remote objects that determine perceived position ( $p$ 92)

With a simple problem such as that of figure 17 , one of those used in a rat "intelligence test" (Hebb and Willams, 1946), learning takes from 1 to 5 trials In this method the food box is always in the same place, but there are movable barners that are changed for each test, so the rat is used to finding food by a different route each day. With the problem shown, a bright rat is likely to enter the error zone $B C$ once or twice, and then make no more mistakes, following the route $A-D-F$ consistently

At the point $A$, the rat's behavior on the first tnal is determined by his experience with preceding problems. He has sometımes found food by going toward $B$ followed by a right turn, sometmes toward $D$ followed by a left turn, and always 
in the general region of $C$ and $F$. At $A$, accordingly, the phase sequence is variable (unique at any one moment, but changing from one moment to another as "meaning" varres-p 133). W1th each phase sequence, there is a selective influence on the motor system, determining head-and-eye movement toward $B, C$, or $D$ Further, the phase sequence is dominated by two things an anticipatory concept or expectancy of reaching food, and perception of the rat's present place in the maze Each two conceptual complexes are linked by activity of the cortical motor system facilutating not only movements of the head but also locomoton The latter facilitation is not always liminal, but when it is liminal the rat runs, in the direction determined by the earlier movement of head and eyes

The sequence of events, as the rat reaches $A$, might then be as follows perception of $A$-expectancy of food, with onentaton toward $C$-running-perception of $C$-expectancy of an alley leading to $F$. No alley appears, and exther of two things may result.

1 The first possibility is that the controlling phase sequence is not strongly established and readily gives way to another which determines a different direction of movement. Expectancy vas not confirmed, the ongunal phase sequence has not been remforced by a corresponding sensory sequence, and the probability of tts occurrence when the rat next arrives at $A$ is-at most-not mcreased On a second tral, the run may be toward $D$. Now the expectancy of an alley beyond $D$ is reinforced sensorily. The individual phase in the phase sequence is aroused - both by a sensory and a central facilitation, strengthening interphase facilitations and so mocreasing the probability that on a third trial the rat will go from $A$ direct to $D$

2 As the rat approaches $C$ on his first run, the second possibulity is that the phase sequence determining this behavior is strongly established, with a strong motor facilitation. The end of the cul-de-sac not only fals to provide sensory reinforcement for the phase sequence but disrupts it There is a conflict between the original phase sequence and a new one aroused by contact with the end of the cul-de-sac, which has been associated in earlier experience with a night-about turn. This confluct may appear as an emotional disturbance (anticipating the 
discussion of emotion in the following chapter), and amounts to breaking up a link in the chain of the recurrent organization of the phase sequence In succeeding trials when the rat reaches the point $A$ in the maze, the phase sequence may be, as before, perception of A-expectancy of food, with orientation toward $C$-anticipation of $C$ But the anticipatory concept of $C$ is now linked with a disruption of cortical organization. The cyclical series $A-C-F-A-C-F$, and so on, is broken up, permitting another cycle $A-D-F$ (origunally less strongly established) to occur instead.

This, in outline, is the approach to adult learning that is suggested by the phase-sequence hypothesis As I have said, there are large gaps in the evidence, and when these are filled in the hypothesis may be found to need senous modifications It has not made use of motivation or insight as special processes independent of the phase sequence, because these with emotion seem already subsumed by the hypothesis I shall ty in the following chapters to show how such processes may be dealt with in more detal. 


\section{Higher and Lower Processes Related to Learning}

The course of learning is rarely a smooth accession of motor skill, or a closer and closer relationship of response to some particular environmental event, as the learning process goes on. Learning curves, for the individual animal, show great fluctuations from day to day $\rightarrow$ now backsliding, now sudden improvement It is only by averagung the results from a number of antmals, trial by tral, that one can manage to get a simple curve showng steady improvement with practice

It is usually considered that these fluctuations are caused by a number of factors that are independent of learning and of each other. Just what factors are invoked depends on the ind1vidual writer and his theoretical point of view In general, they mclude (1) processes that guide and strengthen learning: physiological needs and motivations (in one kud of theory), or such things as attention, expectancy, and insight (2n another), (2) processes that weaken or disrupt leaming, such as emotional dusturbances, and even (3) the innate processes of instinct that are thought to take the place of learning

The question of differences in the learning of dufferent species has already been broached. Amplifynng this topic will also serve to fill in some of the more umportant detals in the account of learning that has been outlued in preceding chapters In the present chapter, accordungly, the "higher" processes of consciousness and insightful behavior will be discussed together with the "lower" factor of instuact, and, more briefly, the relaton of these to emotion and motivation, leaving a fuller account of the latter processes to following chapters. 


\section{ENDURING SELECTNITY IN CENTRAL ACTION ATTITUDE}

An important aspect of an anumal's behavior is the occurrence of penods of directed behavior, in which one kind of action appears only, or in which responsiveness is to one kand of stimulation. This selectuvity appears to be like the selectivity of attention but lasts longer and is less spectic. It might be referred to as attitude, interest, or intention (though these terms may be objectionable), to distanguush it (1) from the more briefly lasting attention, and at the same tume (2) from the stable selectuvity that may be either inborn (and referred to as instinct) or establushed by slow learning, and remauns unchanged from one day to the next The behavior discussed here is behavior in which responses are now to one class of strmulus, now to another, in the same environment.

At a purely descriptive level, this aspect of behavior must be considered when one ettempts to deal with species differences. It seerns first that the duration of the selectivity-the amount of tume likely to be spent contmuously in one kand of activity-may vary for higher and lower species Periods of spontaneous play are shorter in the rat than in the dog, and still shorter than in man, the penod of emotional disturbance followng momentary stimulabon seems progressively shorter as one goes down the phylogenetic scale, and so on Secondly, the number and variety of the distnctive forms of behavior unvolved is greater in higher species-the number of recognizably dufferent attutudes or interests, that is, is greater. And, finally, the varabiluty of action that still remalns selectsve is also greater in higher species: an aggressive attudude, for example, has a much more variable expression in the chmpanzee than in the dog.

At a theoretical level, it seems further that there can be no explanation of learning and problem-solvng in any mammal without reference to the persisting central neural influence that sustains activity in one particular durection. Even in the rat, leaming continually shows this selective responsiveness to one aspect or part of the environment. In higher forms, where expectancy has been most clearly demonstrated, it seems often 
to be organized by an expectancy of a particular reward or goal This is clearly not sensory but a conceptual process (to which, however, sensation must continually contribute)

To account for a series of responses to the environment in which a lasting central influence is evident, it would be convenient to be able to think of reverberation in some thalamocortical system, acting without interruption to influence other cerebral systems in a particular way for extended persods of time But this is at the least unlikely Even if such a system were unexposed to external disturbance, simple reverberation without afferent support cannot be expected to last more than a fraction of a second (Lorente de N6, 1939) The schema of neural action on which the present theory depends has made it appear that there is no constant, uninterrupted activity determining behavior, no one enduring pattern of neural firing, but a continual fluctuation. If the theory up to this point is to be accepted, a selectivity of behavior that may last for minutes or longer must be ascribed to some other limitation on the variabulsty of firng pattern in the cartex How might ths occur?

First, there may be classes of associated cortical activities. Because of having occurred together frequently, each activity in a class $A, B, C, D$, and so on, would facilitate other activities in the same class more than those in another class $L, M, N, O$. Arousal of any one activity $A$ would then arouse others $B-C-D-A \cdots$, but none of $L, M, N, O$. Each letter here stands for a conceptual activity, or phase cycle, not a cell-assembly action The duration of each phase cycle might be a matter of seconds, of the senes of cycles (or conceptual series), correspondingly longer. This would be one mechanism of a persisting attitude, with a mainly intracerebral ongin. Its maintenance might also be contributed to by sensation; the behavior would amount to a seeking out of certain stumulations rather than others, and these might be such as to reinforce the selectivity of further response.

But secondly, if the intation of one of these protracted and selective conceptual series mvolves some lastng bodily condition such as lack of food, which in the presence of a particular environment sets up an expectancy of eatung, the expectancy might dominate the series and have a further organizing influ- 
ence (The assumption is that hunger pangs, or a low level of blood nutrients, would have a selective effect on the lumen for the conceptual actuvities determining eatung. This assumption will be discussed later, in relation to the problem of hunger) If $A$ then stands for the expectancy of eatung, and $B, C, D$ for conceptual activities associated with eating, the senes might be $A-B-A-C-A-B-D-A$, and so on, with $A$ in a domnating role. Thus a lasting bodily state might repeatedly induce a conceptual activity whose frequent recurrence would act to organize a conceptual series and restrict its content This second mechanism presumably would make possible a longer duration of attitude than the first alone.

The duration of a self-mantanned activity, as contrasted with an activity that is frequently remstated sensonly, depends on the complexity of the system in which it occurs. A simple closed circuit may reverberate for perhaps 0001 to 005 second; the cell-assembly, in which an alternating reverberation is possible (by-passing refractory transmission units), has been assumed to be capable of activity for periods as great as half a second (p. 74); a phase cycle, or sequence of recurnng assembly actrons, might then endure for perhaps 1 to 5 or 10 seconds (the apparent duration of conceptual processes in man). I have assumed in the precedung paragraphs that a series of such cycles or concepts might last for minutes or longer and constitute a transient attutude. A greater complexity does not ensure a longer persistence of self-maintained actıvity in the system, but may permit it, and at the same tume it permits or depends on a greater variability of specific content from moment to moment. In the conceptual class $A, B, C$, and so on, $A$ facilitates $B, C$, and $D$. A temporanly high limen in $B$ at the time of $A$ 's activity would not prevent contunuance of selective behavior, since $C$ or $D$ may be aroused by $A$ if $B$ is not. The idea in essence is that a conceptual series $A-B-C-D-A-B-C-D$, and so on, might hnve a greater duration, at its maximum, than $A-B-C-A-B-C$, and so on.

In a higher brain, accordingly, in which more assemblies are organuzed by learning and may occur in more varied combinations, the upper time limit of a selective central mfluence is greater and the selectivity at any one moment is less specific 
The anatomical factor here is both absolute size of the association areas and the $A / S$ rato (p 124) This provides some rationale for the observed relation between (1) phylogenetzc level and (2) the complexaty and duration of atthude and interest. The problem dealt with here has not of course been solved. It will be returned to in Chapter 9

Now the behavior that shows this sort of control is the behavior that is called conscious or voluntary. These two terms are not in good standing in modern psycbology, and yet they refer to an important psychological distnction not only for the clwac, where it is fundamental, but even in the expenmental laboratory, where for example the differences in conditioning voluntary and involuntary responses are well known It is not important whether one is to contunue using the words conscious and voluntary in psychological discussion, it is important to have the distinction to which they refer made explicit, and to be able to deal with it theoretically.

Let us start by recognizing that the distinction is not between discrete, unrelated states but between the extremes of a contunuum. The important thing is to define the contrauum, not to decide just where a line should be drawn to divide one end from the other, or even to divide it at all.

The preceding discussion of attitude makes a thooretical defnition of "voluntary" and "conscious" possible, and, just as important, draws attention to what seems to be the empincal meaning of these terms, quite apart from theory. For the present theory, a voluntary act is one that is determmed by a phase sequence or conceptual series with some duration in tume, to which both sensory and central faclutations contribute constantly. These sequences are recurrent as well as anticipatory, so the voluntary act is influenced by immeduate memory and set as well as by an expectancy of the future-particularly, an expectancy of the immediate consequences of the act. This theoretical defintion is considered also to cover the adjective "purposeful"-from the point of view of mechanesm, there is no apparent basis for distingushing "voluntary" and "purposeful" (the distunction in usage may actually be a distunction between the circumstances in which a single knd of process occurs).

Empirically, a voluntary act appears during penods of selec- 
twe responsiveness, conditioned both by earlier stumulations (the influence known as set) and by present stimulation. The influence of present stumulation is not always obvous, since the act is not necessarily inituated by an environmental event. It becomes evident, however, whenever an environmental change $\alpha c-$ curs during the act that would cbange its effect. There is at once a vanation of muscular pattern that is such as to tend toward a constant end effect. This muscular variabulity with a single result has been called "motor equivalence" by Lashley (1942a) It is an essentral mark of the voluntary act (and is essentially the only meaning of the adjective "purposeful").

The form of an involuntary act is characteristically not a functon of set, or any preparation of the subject by instruction or the like (Hulgard and Marquis, 1940). It is determined by the stimulation of the moment plus the general physsological condition of the animal, and does not show motor equivalence. The occurrence or strength of an involuntary response may be subject to the influence of set, as when ghost stories faclitate a startle reaction, but the startle is involuntary as long as it maintains the characteristic nigidity of pattern The distinction is of course between two ends of a continuum, not attempting a sharp separation of two totally dufferent kinds of behavior Some involuntary acts may show "purposeful" motor variabilty, or vary in pattern as a function of set, but the more they do so the less certain the classification must be.

Conscrousness then is to be identzied theoretically with a certain degree of complexity of phase sequence in which both central and sensory facultations merge, the central acting to reinforce now one class of sensory stumulations, now another The cortical organization consists of a duffuse firng in a succession of assembly actions or phases that are organized first in phase cycles (conceptual activities) and then in series of such cycles that maintan a selectrve influence on behavior for appreciable pernods of time, but always with the possibility that the current organization may be changed or disrupted by an unusual sensory event. The limen for the unusual, that is, remains low. The unresponsiveness to some stumuli that is characterustic of the conscious anunal is always for famliar features of the environment. Funally, this organization is opposed to the intrinsic or- 
ganization of infancy and sleep (p 121), and appears to be maintaned by the constant sensory flux to which motor activity contributes. It is rarely possible for any prolonged period of unchanging sensory pattern (when sleep or catalepsy is likely to ensue).

Empuncally, in behavior, the most mportant mark of consciousness is the continually changing selective responsiveness to different aspects of a famllar environment, the unchanging responsiveness to unusual or unexpected events, together with the contmual presence of "purpose," "means-end-readuness" (Tolman, 1932), or motor equivalence. Another mark might be the occurrence of "operants" (Skanner, 1938), or actions not inithated by an envronmental change. However, these occur also in earliest infancy, in very primitive organisms, and in sleep, and so do not very clearly distungush a conscious from an unconscious animal.

\section{MODES OF SENSORY-CENTRAL INTERACTION}

The chief need left by this discussion of attitude, or enduring selectivity of central action, is a more detaled treatment of the interplay of sensory and central facilitations. I have sard that the phase sequence persistently escapes from a durect sensory control. But this by no means mples a total lack of sensory influence, at any time. By "direct" control is meant that assocration-area activity is determued by the pattern of immediately preceding sensory stimulation, sunce this activity determines bebavior, a durect control would mean that with any given sensory stumulation an anumal would respond in only one way. Thus, as we know, does not occur in hugher animals in a familuar environment At the same time, it is quite evident that there is a contrnual influence, of some knd, from sensation.

Defining this influence in its vanous forms ranses a number of problems. The schema of neural action made the mode of sensory-central interaction explicit as far as momentary excitathons are concerned, but new difficultues appear when one is dealing with lasting central activities and a series of sensory events Some of these are handled reasonably well in the phasesequence bypothesis. Others remain as problems for exper1- 
mental investigation. They can be defined here and at least re lated in a general way to the hypothesis

At each point in a conceptual series, the ensuing activity is determined by the total pattem of sensation at that moment and by the residue of facilitation and negativity from the preceding central activity In general terms, this means that there are three possible ways in which sensory and central facilitations may interact. (1) They may confluct, producing phase sequences that are mutually incompatible; (2) they may have unrelated effects, tending to set up independent phase sequences in parallel, and (3) they may reinforce one another's acton, both facilitating the same subsequent pattern of cortical action

The second and third of these possibilities are the subject matter of later sections, dealing respectively with the relation of attention to learming, and the role of expectancy in a skulled motor performance The first possibility, a confict of faciltatrons, is related to the problem of emotional disturbance

\section{\ THE RELATION OF EMOTIONAL DISTURBANCE TO LEEARÑNING}

The original 1dea of emotion was probably a reference to the demons that now and then disturb ordmary behavior Demons or no, let it be observed that this was an inference from behavior, demons (including the soul itself) not being easily seen by most people.

Very much later a quite different idea appeared, and is still current. Emotion is now generally thought of as an awareness, a distinctive conscious process that is quite separate from intellectual processes. This notion has led to a good deal of confusion, for it has gradually become clear that no such distinct kund of awareness exists (a surprising unanumity of psychological opinion has been reviewed elsewhere-Hebb, 1946a) In this sense, the term emotion has lost much of 1ts meanung Further, it is meaningless as referring to any momentary muscular or visceral response, for here also no distunctive pattern can be found (Dashell, 1928).

But it is still true that emotional disturbance exists, and can be recognzzed as a long-term deviation from an anmal's ordi- 
nary behavior (Hebb, 1946a) Somethung causes it. I propose that we return to an earlier, behavioral, interpretation (but substituting neural events for the demon), and define emotion as the neural process that is inferred from and causes emotional behavior, with no reference to consciousness However, for reasons to be considered later (Chapter 10), we shall be concerned in the present chapter with emotional disturbance rather than emotion

Now as to mechanism The causes of rage (Hebb, 1945a) and fear (Hebb, 1948b) are extremely diverse Unless we are to suppose not only that there are a number of separate emotons, with independent neural mechanusms, but also a number of angers and fears, each equally independent, we must ask what these various causes can have in common

One answer to this question has already been suggested. Emotion may be a disruption of cortical organization, which could occur in several ways: the occurrence of incompatible phase sequences, the absence of a sensory faclitation that bas always contributed to the phase sequence, "pain" stimulation that can be supposed to be innately disruptive of cortical activity (see Pain, Chapter 8); and chemical change of the blood content, altering the rate of fing of individual neurons and so disruptung a cortical organization that is fundamentally a matter of tuming (see the discussion of hunger and addiction, Chapter 8)

Some of the points involved must be left for later chapters. For the present we are concerned with the possiblity of a sensory disturbance of the phase sequence, as an unfluence on learning In the schema of neural action, it was concluded that a neuron, or group of neurons actung as a transmission unit, must be expected to enter into more than one assernbly of the assemblies are organized at separate trmes. In two assemblies involving some of the same transmission units, a simultaneous activity would not be possible. The activity of one would prevent activity in the other

In assemblies of cells that are frequently active at the same time, fractionation eliminates from each the transmission units whose action is unterfered with or controlled by others (pp 88-9) In a famliar environment, therefore, such conflict would 
not usually occur The same process would also gradually elimtnate confluct occurring at the first presentation of a new stimulus combination, as the combination is repeated, the make-up of one or both conflicting assembles would be changed by fractionaton, and the disorganization would decrease The strange combination would now be familiar and no longer disturb behavior.

In the phase sequence, the central faclitation from one phase on the next is usually not specific, since sensory events occur in a variable order, and, because of this, the faciltation from one phase cycle or conceptual activity is not completely specific but tends to arouse one of a number of subsequent activites (pp. 133-4) This flexibulity of the phase sequence permits a considerable vanation of the sequence of sensory events without a disturbing effect Such considerations do not permit one to say how great an envuronmental change must be to produce an overt disruption of learned behavior, but the assumption that disruption of cortical organzzation may vary in extent and in duration, and the assumption that the disruption when it has more than a momentary duration means some incoordmation of effectors, make sense of the emotional disturbance that appears when envuronmental change is great enough What I postulate is that, up to a certain point, lack of correspondence between expectancy and perception may simply have a stumulating (or "pleasurable") effect (Chapter 9); beyond this posst, a disruptive (or unpleasant) effect.

The changes that disturb behavior must also include the absence of a customary stumulation The phase sequence is a contunual interaction of central and sensory events, and the background fachitation from a constant sensory faciltation must affect the composition of the cell-assembly, dunng its organizaton, and be a factor in its later arousal The absence of a customary stumulation wll be "strange," and exciting But again, if this condition is repeated, a reorganization of assemblies and change of interassembly faculitations can re-establish the stabulity of the phase sequences concerned With a varned experzence, the animal will become less and less dependent on any particular stimulation that is not a constant feature of his envronment.

Thus is an effect of learnung on emotion, a mechanism of 
adaptation to the strange. The converse is a durecting influence of emotional disturbance on learning, a problem rased in the preceding chapter. It has been concluded that the prompt learning of maturity depends on a phase sequence with a recurrent organization. Disruption of any lunk in this disrupts the whole. Hypothetically, the disturbance vares in extent when it is widespread, it may cause deterioration of other, alternate, phase sequences-an extreme instance, presumably, being the paralysis of terror. With less marked disturbance, the effect may be to prevent activity in the current phase sequence only, and so allow another mtegrated sequence to take its place. In this case, there would be little sign of emotion in the behavior, although the disturbance would have its effect in a changed durection of response

This is supposed to be the way in which emotional disturbance gurdes learning An example will make the proposed idea more definite Let us suppose that a rat is being trained to avord a darkened door, by being given an electric shock, and being allowed to find food when he goes through a lighted door As the rat makes his choice, suppose also that his perception is of the relation of the nght-hand door to the distant environment, the relative brightness of the door not arousing a conceptual activity He approaches the right-hand door, and receives a shock The disruptive effect here is on the conceptual series: perception of present place-door A-expectancy of fooddoor $A$, and so on Back at the chosce point, an alternate series mvolving perception of door $B$ can displace the first, the rat. goes through $B$, and finds food The effect of this one tral is to teach the rat to go through the left-hand door. Unless there was a conceptual activity aroused by the different hightung of the two doors (p 106), this trial would not set up a tendency to avoid a darkened door as such.

Subsequent trials, with the dark door sometmes right and sometimes left, would accumulate a disruptive effect on both "spatral hypotheses." But on any trual when the conceptual content includes phases determmed by the brightness or darkness of a door, an association of darkness with shock can begin to be established. With this, the phase sequence will alternate between perceiving present position at the chouce point-per- 
Intention and Attention as Factors in Learning 151

ceiving a brighter door-expectancy of food, and perceiving present postion-percetoing darker door-expectancy of food. The first is reinforced by a corresponding series of sensory events, as the rat runs under its influence, the second is unreinforced, and, further, disrupted. When this stage has been renched, the final leaming of the task should be prompt.

This, and the discussion of frustration in the preceding chapter (pp 138-9), represent in prnciple the role of emotional disturbance as it might affect learning in an expermental study. A special relationship between emotional disturbance and learning will be dealt with in Chapter 10, which tries to show how learning might develop typical rage and fear, with theur distinctwe patterns of organized response, out of what ( $m$ its ongins) is sumply disorganzation.

\section{INTENTION AND ATTENTION AS FACTORS IN LEARNING}

We can now tum to the second way in which sensory events may be related to the current central process The first possiblity, just discussed, is that the two may conflict. The second is that they may neither confluct nor reinforce one another, but determure independent phase sequences. This possibulity bears directly on the commonly held opinion that no learning is possible without intention to learn, no memory of a sensory event unless it was "attended to" at the time of its occurrence

It is often supposed also that there is an extraordinary unity about attention-that one can attend to one thing, or to another, but not to both at the same time unless the two are a single unit, responded to as a whole. These conclusions need qualification

There is actually no evidence to justufy the general statement that learning never occurs without the help of attention (though my treatment in the following paragraphs assumes that this is in a sense true). It has been shown repeatedly that a subject may not remember what he did not attend to, or that he remembers more with attention than without. But other things are apt to be remembered to some degree. It is common experience to remember details of a sttuation to which one did not, appar- 
ently, attend-when the details later have become relevant to one's thought. Similarly, the remark made by someone else, when one is reading, may not be noticed or responded to in any way, as far as anyone can tell, and yet 30 seconds or a minute later one becomes aware of the rernark and answers it The event was remembered Are wo to postulate an "unconscious" attention to explain the fact? If so, attention is no longer unstary-even if there were no other objection to such a treatment of the problem.

This is a point, it seems, at which such theoretical conoeptoons as attention and intention are no longer precise enough. I have already defined attention as the immediate facilitation from one phase, or assembly action, on the ensuing one, with no implication that two such processes might not occur at the same time. In so far as the term is to be useful, it should be restricted to this very brjef central faculitation. "Intention" seems a still more slippery term, related to "atthude" but with the implication that motor components are prominent If the term has any intelligible reference to neural function, it must be to the enduring selectivity of central action that was discussed earlier.

There is a certain unity about this action, but not at all the absolute unity with which attention, at least, has been endowed in the older hiterature. There may be more than one phase sequence in the association areas at one time The discussion of meaning in the preceding chapter implied also that the phase sequence may conturually tend to branch out in more than one drection, ameba-like-some, or all but one, of the branches eventually becoming aborthve for lack of the general background facilitation of "attitude" that determines the general trend of the phase sequence. I have emphaszzed the idea that the motor faclitation of phase or phase sequence may be subliminal, and, to the extent that attention has the unity it is supposed to have, the unity may consist of a control of the motor_system by one phase sequence only, when two or more run in parallel Even then it should be remembered that we very often carry on two familar activities at the same time. Arguing and driving a car will do as an instance, netther seems possible without "attention" It certanly seems that the unity of attention has been exag- 
gerated, and the phase-sequence hypothesis, at least, suggests that it may often be multiple

Memory for an incidental stmulation then may occur because the stumulus has aroused a second phase sequence without interrupting the prior dominant one This can happen only with familnar activities and perceptrons, and only if a phase sequence is aroused by the stmulus in question; and, in agreement with the importance of meaning for learning, only if the mosdental phase sequence has a certain degree of complexity and persistence There will consequently be plenty of occasions on which "unnoticed" events are not remembered, as the expenmental literature has shown

So also with the effect of set on perception (perceptual set is another aspect of the phase sequence, closely related to attention) It has been shown that in certain circumstances one sees what one expects, or is set, to see But this is no general rule, else one would be mcapable of seeng the unexpected. When a stimulation is sufficiently strong or distenctive from its background, and familiar or composed of familar parts, it will be capable of mitrating its own phase sequence, regardless of what other phase sequence may be gorng on at the moment (If the new phase sequence is meompatible with the pre-existung one, startle or emotion may result ) The sensory event is capable of taking control of central action, just as in other circumstances the central action is capable of remforcing one sensory event at the expense of a second

\section{MOTOR EQUIVALENCE}

Two modes of sensory-central interaction have been discussed The first, a conflct of sensory and central processes, was regarded as a source of emotion, the second, in which a sensory event has central effects that neither support nor conflict with a prior central process, was treated as accounting for certain kunds of incidental or unintentional learning.

The third mode, an active support and direction of the phase sequence by sensory processes, is at least as important. It is best exemplified in the phenomenon of motor equvalence. a vartablity of spectic muscular responses, whth carcumstance, in 
such a way as to produce a single result. The phenomenon and its unportance for the theory of learning have been emphasized by Tolman (1932), Hull (1934), and Lashley (1942a) At the same time, theory has not handled the problem very successfully. It is usual, and in practice necessary, to define an antmal's response in terms of its end effect the rat reaches and enters the food box, the chimpanzee's hand makes contact with a stimulus plaque and moves it It would be umpractical to record the actual senes of muscular contractions that brings such an event about But one must not then assume that a common end result means a single pattern of contractions.

It is necessary to use very special conditions of training indeed to establush a "learned response" as a constant effector response A rat trained to depress a lever to get food may do so from any of several positions, in each of which the muscular pattern is different. He may clumb on the lever, press it down with the left forefoot, or the right; or use his teeth instead. Very often, all that can be predicted after the response is learned is that the lever will be moved downward. It is not necessary here to multiply examples of such behavior, it will be evident to anyone who cares to watch anmal or man carry out a learned response With long overpractice it may seem at times that the subject has settled down to the use of a specific muscular pattern, an exact duplication of his movements from one trial to the next. When this happens, however, it will rarely be difficult for an experimenter to change that pattern and still observe ap; proximately the same end effect (Tuming, however, may be disturbed.)

In general, the variabulity of motor pattern in a hungry animal's reaching food becomes comprebensible if it is supposed that the behavior is determined both by an expectancy of the ummeduate result of each movement and by a contmual perception by the animal of his own locus and posture at each stage of his response. The problem has sometimes been discussed as though the motor variability occurred despite a constant set of stumulatung condutions Sometumes, indeed, it may, when there are two or more equally efficient modes of solution avalable to the anumal But the varrability characteristically appears when 
there is a vanation of the anmal's initial posture, of his locus with respect to the goal, or of intervening barners

The animal must perceive his own posture and his place in relation to the goal, and his behavior must be affected by this perception as much as by hus anticipation, expectancy, or foresight of the state of affaurs in which food has been found in the past (remember that we are discussing the variability that $o c-$ curs after leaming is established) The monkey that must lift the hd of a box to get food has an expectancy of the lid's rising in contact with his hand, and be knows where his hand is at the moment. The question then is, what neural mechanism will account for a movement of the hand direct to the edge of the lid?

Here we have an example of motor vanability in a learned response that is about as simple as can be. The muscular pattern of response, when the hand moves direct to the hd, is obviously different for each intual position of the hand We cannot, I believe, accept the solution of this problem proposed by Hull (1934), in his concept of the habit-family hierarchy, for he assumes that the final muscular event in the sequence is constant, though earlier ones can vary Here even the final pattern of contractions is variable, depending on the monkey's position with respect to the box, and we know that the monkey will open the box despite changes of distance from the retaining cage or changes of the box's onentation

But we can follow the general line of Hull's argument and assume ( $I$ believe, necessarily) that an earlier generalized learning of infancy provides the basis of response, together with an expectancy by the anmal of his own later movements. The only thing that seems common to the various responses is a perception of the hand in final contact with the lid of the box In the normal primate, this perception certanly involves vision. I assume thus that the movement of the hand primarly follows an eyemovement that fixates the point of contact. Part of the learning of infancy must establish an ablity to move exther hand or foot durectly from any point to the line of vision Given this, the behavior is explained, my earher schema of neural action having provided for eyemovement in perception. 
The schema has also shown how the cortical process controlling eyemovement might be subluminal for actual movement and yet have a facilitating action on other cortical processes The first learning involving manipulation, in the normal primate, would be accompanied by eyemovements; but later, in man at least, there is a capacity to move the hand to the point one only "thinks" of looking at. In this case it is assumed that the motor process is subliminal for eyernovement, but still with an integratung function for other cortical processes $\mathrm{My}$ account is explicitly in terms of vsual processes, but I will add that the arguments applied (in the earlier schema) to eyemovement might as easily have been applied to movement of the hands, so this does not mean that the variable motor behavior of the congenitally blind is unaccounted for, or different in principle from that of the seeing. Further, there is plenty of evidence to show that the space perception of the congenitally blind is radically defective (Senden, 1932)-which justfies an emphasis on the role of vision in the manipulative actuvity of normal subjects.

In the specific example discussed above, the hungry monkey might equally raise the lid of the box with either hand, with a foot, or even with a stuck. all that is necessary to account for the behavior is that the foot, or the end of the stick, be seen in its present position, that its end position in contact with the lid be conceived of in advance, and that the monkey have leamed to move it from one position to another presently being looked at.

More complex patterns of manipulation requure only a number of such steps a continual awareness of the present position of the hand (or foot), and at each point in its movement an anticipation of the next point in space to which it must be moved. At all tumes the behavior is under the control of these two things, the muscular pattern of the half-grown or adult animal is so variable sumply because the subject is not learning now to make specific movements (as he is earlier, perhaps, when he is learning to bring the hand to the line of vision), but learning a relationship, an association, between perceived environmental events This makes adult learning primarily perceptual, but, in accordance with the developmental changes of learming discussed in the preceding chapter, it still leaves a great emphasis 
on the motor learning of infancy, which frequently can be regarded as purely sensorn-motor.

In this section I have thed to present my solution of a ubiquitous, fundamental psychological problem. It would be wrong to leave the impression that the solution can be regarded as complete. The solution says in short that motor learning at maturity is perceptual learning in the first place. It also says that a true motor leaming of infancy is an essential element in the equivalence of adult responses. The adult who learns to operate a slot machine learns, that is, that a quarter's moving into and falling down a slot will be followed by the appearance of a package of cigarettes. He Jearns that the depression of a certain lever starts the motor on his car. He does not learn to make a certain series of muscular contractions, but, once the perceptual relationship between depression of the lever and starting the motor is learned, an earlier infant learming is enough to provide for the adult's being able to put a hand or foot-or a stick $-2 n$ contact with the lever and depress it. But all thus is in very general terms.

I believe that such conceptions will be a good basis for the study of motor learning, but it should be emphasized that the study is stull needed-that nothung more bas been provided than a rational approach which may or may not be found adequate in the long run. The discussion has said nothing about the changes of skill with practice What is their nature? Are they to be comprised in the conception of changes in the cell-assembly due to an increased frequency of activity? Does the subject revert to his first clumsiness of performance when he is required to use a new muscular pattern? Does his perception of the stuation change with practice, or his muscular tming-or both? Above all, is he still responding to the same cues?

We still have a great deal to learn about the modufication of motor performance with practice It should be said exphcitly that this discussion has not attempted to cover the development of motor skalls involvng a precisely timed senes of movements, nor such learning as that demonstrated by Sperry (1947), which monkeys are capable of and rats are not. 


\section{INSIGHT AND HYPOTHESIS}

A somewhat different aspect of the problem of learning, and its phylogenetic correlations, brings us to the question of intelligence or msight in learning, and the related question of "hypotheses" in animals. These ideas have occasioned a good deal of dispute, and some confusion, in the psychological literature.

The analysis of animal intelligence, by controlled experiment, has been a slow process. Mainly, the trouble has been to make the transition from an earlier subjective psychology (resorting freely to the notion of "mind" or conscious awareness as an agent in behavior) to an objective theory of neural action, without oversumplifying the facts

If an animal shows "intelligence" $\mathrm{m}$ his learning and problemsolving (Hobhouse, 1915), "1deation" (Yerkes, 1916), or "insight" (Kohler, 1925), can the fact be dealt with in a stmctly monistic theory with no hidden thread of anmism? It was hard to find a way of doing so What psychologists really needed, of course, was a physiological mechanism of thought to supplement their frankly physiological treatment of afferent and efferent mechanusms. The fallure to suggest any intelligible mechanusm of msight, or ideation, led to two sharply opposed points of view One group-in general, those I have called learning theoristsbegan by denying that the facts were what they seemed, and argued that insight must be reducible to simple learning

The other group-in general, the configurationusts-proceeded by urging the need for a better theory of learning, whether it could be found at once or not, and contunued a search for evidence to show that the need existed, that anmals do have ideas, or insight, or intelligence.

As a result of this undertaking, psychological knowledge made a considerable advance I am going to argue that such notions as insight have a limited value, and that something with more precision will be needed for the further development of theory, but it should be recognzzed that these ideas have led to an unproved analysus of animal behavior and its qualitative variations at dufferent phylogenetic levels 
Besides Hobhouse, Yerkes, and Kohler, already mentioned, Lashley (1929a, 1938b) and Krechevsky (1932) should be listed as making major contributions to the defficult problem of analyzing anumal intelligence, and Tunklepaugh (1928), Tolman (1932), and Cowles and Nissen (1937), as well, for demonstrating expectancy (a form of ideation) in animals and establishing the value of this notion in the theory of behavior

It is generally conceded by now that learnug involves more than the gradual reinforcement of responses ongunally made at random. "Sophisticated" learning theory recognizes the fact as much as configuration theory (Spence, 1938, 1940) Learning is often discontinuous, error curves show sharp drops without warning, and the kind of error that is made on one day may be quite changed on the next. The great disagreement that stall contenues in psychology does not concem the exustence of some factor producing these effects, but its nature. how it operates, and how it is related to learning as such

Let me make thrs a little clearer. In the first efforts to be nd of anthropomorphism in animal psychology, "learning" theory sard something like this When an animal is faced with a new problem, we can avoid any reference to intelligence or comprehension and describe the cat's attempts to open the door of a problem box (for example) as at first a series of random movements. Some one of these movements will eventually open the door, by chance The tendency to make that movement is then increased because it was rewarded (the "law of effect").

On each tral, the tendency is further moreased. Eventually, the cat opens the door immediately-not because of any separate process of intelligence or understanding, but because a specufic association has been built up This is the "naive" theory of learning by trial and error, it is no longer mantamed, largely because of the work of Hobhouse, Yerkes, Kohler, Lashley, and Krechevsky, together with an umportant analysis by Adams (1929) and the later work of Thomdike (1931) But it had its value It was a necessary corrective to the vagueness of other theories, and though in development it has become rather vague itself it still represents a line of thought that insists, nghtly, that theory must aum at definable conceptions and a clear statement of its original assumptrons 
Besides Hobhouse, Yerkes, and Kohler, already mentioned, Lashley (1929a, 1938b) and Krechevsky (1932) should be listed as making major contributions to the defficult problem of analyzing anumal intelligence, and Tunklepaugh (1928), Tolman (1932), and Cowles and Nissen (1937), as well, for demonstrating expectancy (a form of ideation) in animals and establishing the value of this notion in the theory of behavior

It is generally conceded by now that learnug involves more than the gradual reinforcement of responses ongunally made at random. "Sophisticated" learning theory recognizes the fact as much as configuration theory (Spence, 1938, 1940) Learning is often discontinuous, error curves show sharp drops without warning, and the kind of error that is made on one day may be quite changed on the next. The great disagreement that stall contenues in psychology does not concem the exustence of some factor producing these effects, but its nature. how it operates, and how it is related to learning as such

Let me make thrs a little clearer. In the first efforts to be nd of anthropomorphism in animal psychology, "learning" theory sard something like this When an animal is faced with a new problem, we can avoid any reference to intelligence or comprehension and describe the cat's attempts to open the door of a problem box (for example) as at first a series of random movements. Some one of these movements will eventually open the door, by chance The tendency to make that movement is then increased because it was rewarded (the "law of effect").

On each tral, the tendency is further moreased. Eventually, the cat opens the door immediately-not because of any separate process of intelligence or understanding, but because a specufic association has been built up This is the "naive" theory of learning by trial and error, it is no longer mantamed, largely because of the work of Hobhouse, Yerkes, Kohler, Lashley, and Krechevsky, together with an umportant analysis by Adams (1929) and the later work of Thomdike (1931) But it had its value It was a necessary corrective to the vagueness of other theories, and though in development it has become rather vague itself it still represents a line of thought that insists, nghtly, that theory must aum at definable conceptions and a clear statement of its original assumptrons 
The configurationist attack on the earlier, "nauve" learning theory proceeded as follows A search was made for tasks to set an animal, of just the right degree of difficulty for each species to demonstrate the operation of intelligence The task must nerther be so easy that the anumal solves the problem at once, thus not allowing one to analyze the solution, nor so hard that the animal fals to solve it except by rote learning in a long series of trials.

With a problem of such borderime difficulty, the solution may appear out of a blue sky. There is a pernod first of frutless effort in one direction, or perhaps a series of attempted solutions Then suddenly there is a complete change in the directon of effort, and a cleancut solution of the task. This then is the first critenon of the occurrence of insight The behavior cannot be described as a gradual accretion of learning, it is evzdent that something has happened in the animal at the moment of solution (What happens is another matter)

The sccond, and essentially more important critenon, involves a comparison of species To the human observer, a monkey's efforts to get at food may at tumes appear utterly stupid. It is one of the great achrevements of modern psychology to show that the stupidrty is always a matter of degree, and to make a beginning at saying what intelligent behavior is, in its various manufestations. The description of intelligence is a more dufficult task than it sounds, this is one matter in which appearances are really deceptive The box-stacking of the chmpanzee, for example, is a very clumsy performance indeed. The chimpanzee has no notion of statics and must pile his boxes by tral and error until finally they will stay piled and bear his weight (Kohler, 1925). But comparison with other species reveals a considerable intelligence in this performance, nonetheless. The dog is not low in the intellectual hierarchy, and yet he is unable to see the possibulity of moving even one box to a place where he wants to climb Physically, this is possible for him, though piling one box on another may not be. The failure is thus intellectual.

The dog, again, shows his great superionty to rat or hen in hus skill at passing obstacles, and reaching a goal by the shortest route (psychologically a more brilhant achievement, more insightful, than it may sound) 
It is often said that most human beings never thunk, and go by habit instead of intelligence. The dullest person who can earn a living in this culture, however, contmually functions at a level well above the chimpanzee's-complex as the chimpanzee intelligence is-and it becomes nonsense in view of this fact to suppose that a man could ever get along solely by routne without the continuous operation of whatever it is that we call intelhgence. This becomes even clearer when one looks at cases of aphasia. As we shall see later, aphasia is by no means a mere loss of speech, the patient has lost something else as well, an intellectual capacty that no chimpanzee will ever have and one that every normal person has and uses continuously (even if he never sits down, puts his chin in bis hand, and announces in so many words that he is about to "think"). Without the study of insight in animals, we should not have been able to see how much is involved in ordinary, everyday behavior that seems elemental in its simplicity but is not.

There is an unsuspected degree of intelligence in even the simplest behavior of normal man Krechevsky (1932) established a sumular point for the rat, showing that apparently simple learning is more than an uncomplicated acquisition of stumulusresponse connections, it involves "hypotheses" as well

Briefly, what Krechevsky found is this. A hungry rat is given a repeated choice of two doors, one left and one night, one white and one black (for example) The white door is sometmes on the left, sometumes on the right, and food is to be found by going through one of the doors (the other being locked). With such a problem, the rat does not choose at random At first he is almost certain to persistently try the door on one particular side, tral after tral, and later, to try the other side in the same way. Stall later, he may alternate, one left, one nght, and so on, or choose always the white door, whichever side it may be on, until finally one of these systematic attempts at solution is consistently rewarded. The rat is then said to have had a series of hypotheses, and it is clear that his learning is not a gradual strengthening of responses that were at first made at random. The behavior is stupid.enough, by primate standards, but it involves intelligence of a kind and it throws a good deal of light on the behavior of higher species. 


\section{DIFFICULTIES OF THE CONCEPTION OF INSIGHT}

So much for the value that psychology has drawn from the conceptions of insight and hypothesis (the latter evidently a form of insight itself) We can next ask in what respect such conceptions are unsatisfactory, and the inquiry will lead us to recognize a complementary value in the "learning" theory that has been consistently opposed to the theory of msight.

From the first the meanung of insight and hypothesis, as exact scientfic terms, has been left confused. Their connotations obnously are anthropomorphic and unfortunate. This might have been less important if the terms had been well defined, but they were not.

"Hypothesis" has been defined as a form of behavior: a set of responses that deviates systematically from a chance performance. This will not do at all, as Prentice (1946) has pointed out; it confuses cause and effect An hypothesis, it becomes evident, is something postulated, hypothetical, inferred from behavior.

Psychology has sometimes in the past been too free in postulating special processes to explain behavior Now it is doing penance by going to another extreme, scientifically just as sinful. This current misconception of the scientific method makes any hypothetical entity somehow disreputable Psychologists now seem to feel that it is risky indeed to depart from statements of fact, nothing should be mentioned that is not "operationally" demonstrable. Thus in another feld we find emotion defined as a boduly reaction, which it is not; or neurosis as a set of neurotic symptoms At the same time, the context in which such definitrons appear makes it obvious that the writer has in mind sornething inferred from behavior, not the behavior itself, just as the rat's hypothesis is inferred It is completely necessary to keep out of such confusion (in the first place; and in the second place, to real uze that nether operationalism nor logical positivism really discourages inference or postulate but only seeks the rules by which inference is to be made)

Insight, again, has on occasion been defined as a sudden change in behavior, or as a conscious expenence that one may 
have in solving a problem. Neither definition gets at the real meaning, which is that of a causal factor in behavior.

When this is clear, it becomes clear also that there is no need to say that a rat has an hypothesis only when he makes a series of systematic choices The hypothesis might endure only for one trial, or part of a tral; though it could not then be durectly detected, it would still exist. In the same way, it is not necessary to limit insight to those occasions such as when a chympanzee, at first faling to reach a banana outside his cage, suddenly turns away, looks for a stick, and rakes in the food These are the occasions on which it is evident that something has changed in the anmal and has led to a solution But if it is insight that solves a problem following error, it is no less insight when an animal makes no errors and sees the solution at once. When the solution is seen at once, there must be at least as much insight as when the solution is delayed

In this sense "insight" is fully equivalent to "intelligence"the term that Hobhouse used. The term insight therefore seems to have two references, one to an enduring factor in behavior, the other to a variable one. Both "insight" and "intelligence" are probably unsatsfactory terms-it will be seen in the following chapter that they may be hard to distmgursh from some uses of "motivation" except as different aspects of the same thing-but it may be suggested that, if they are to be used, "intelligence" should refer to the enduring aptitude, the existence of a causal factor in problem-solving that may or may not lead to the solution of a particular problem, "insight" to refer to its operation in the particular instance.

There is stall a duference of opmon as to what this causal factor in behavior is, and how it operates is insight or hypothesisor, in the broadest terms, intelligence-something distunct from the mechanism of association? And here, I think, we must take a long step toward the position of those theorsts who originally denied the existence of insight but who now, like Hunter (1934) or Spence (1940), are concerned to show that it can be denved from a theory of learning.

Whatever insight 15, we now know that it contunually affects the learning of the adult mammal. This has been mantanned by those who have emphasized the value of insight as a theo- 
retucal conception; if we grant it, the conception does not become more valuable for theory, but less

We find at once the need for more precise conceptions The origmal value of the notion of insight lay in the distunction between intelligent and rote learnung. Now all behavior involves intelligence, and the distanction is a matter of degree how much insight (or intelligence) is present? Further, if all learning is unsightful we can no longer speak of insight as if it were a separate process and distinct from learning.

The conception of insight was a valuable first approximation, but a first approximation only. Insight is not distunct from attention or expectancy (which are also first approximations), just as it is not wholly separate from rote learning. Postulate insight to account for the discontinuity of a sudden improvement in learnung, and sooner or later we shall have to add other postulates (temporary forgetfulness, frustration, loss of interest, and so on) to account for the regressions and disruptions that also occur in learning

It has already been seen how insight, as a sudden perception of new relationships, can result from the simultaneous activity of two conceptual cycles in adult learning (pp 133-4) This need not be elaborated further; the new relationship is a change of the phase sequence, and each phase sequence has its distinctive motor facilitation, so the change would be evident in a discontinuity of behavior-the actual evidence from which the insight is inferred.

Apart from the special case of a sudden solution following fallure, insight is essentully the phase sequence itself, the autonomous central factor in behavior It is that which makes behavior directed, unstead of random, the mechanism that is both anticipatory, adjusting behavior to an obstacle before the obstacle is reached, and recurrent, so that the chimpanzee, trying to reach a banana, remembers the stick he saw a moment before, goes and gets it, and rakes in the frut.

Phylogenetic differences of insight are thus the differences in the complexity of phase sequence that is possible in the brain of a higher or lower species. The.lack of insight in the earliest learnug of primates becomes comprehensible, since the phase sequence must first be organized. 


\section{The Relation of Instinct to Learning}

Also comprehensible is the slowness with which insightful learning develops in these species, by the very mechanism that makes their ultumate level of behavior more insightful than any other. The larger association cortex of promates, both in absolute terms and in A/S ratio ( $p$ 124), means a slower establushment of sensory control, and so a longer tume to set up the phase sequence and insight. At the same tume, in a larger association cortex the number of assemblies of cells that can function as separate closed systems will be larger. This means that the higher animal at maturty will see any particular situation in a larger number of ways The comparatively small sensory pro. jection means also that the phase sequence can escape from complete sensory control more frequently, and for longer penods, so that the insightful behavior of higher species is less a response to the immediate environment and more an integration of sernal stumulation from situations that cannot be surveyed as a whole.

Finally, all this explans how insight depends on experience and yet is not a simple, durect result of learnung, or the operation of specific habits (Birch, 1945). The perception of new relation. ships discussed above is usually not delayed, but immediate, the anumal put into a new situation will perceive its parts as farmilar, and as related in a meaningful way (that is, by the mechanisms discussed on pp 130-4). The behavior is then msightful, as an organized set of responses to a new total situation An organtzation that consists of a new temporal combination of elements which themselves were organzed by experience obviously depends on learning, but is not leamed Heredity sets a lumit to the amount of development of the phase sequence and determines the principles of development, but expenence is necessary as well.

\section{THE RELATION OF INSTINCT TO LEARNING}

The problem of instinct is the correlative of that of intelligence, or insight, and of learning. It has just been seen that intelligence is not an entity that is quite distinct from learning; and we may now see that instinct, also, is not to be cut off sharply from either. 
The term "instnctive" will be used here to refer to behavior, other than reflex, in which innate factors play a predominant part Empirically, this is behavior in which the motor pattern is varnable but with an end result that is predictable from a knowledge of the species, without knowing the history of the individual animal. This class of behavior must be recognized, and we are no farther forward by coinng a new name for $\mathrm{tt}$. "Instunct," however, is less useful. It suggests a special agent in behavior, just as "intelligence," "msight," or "drive" may. Essentrally, we are involved bere in the dufficulties of the constctutronal-experiential dichotomy that are discussed later (Chapter 10) We must distingursh, conceptually, the constrtutional factor in behavior from the experientzal, but there is presumably no mammalian behavior that is unnfuenced exther by learning, or by the constitution that makes some leaming easy or nevitable. We need not consuder (1) that instnothve bebavior is sharply distinct from other behavior, or (2) that it involves some special process that is radically different from those involved in other behavior. Ultimately, our aum must be to find out how the same fundamental neural prunciples determine all behavior.

There is 2 means at hand for attempting thes. Consider first Lashley's (1988a) useful distunction between reflex and mstmct. A reflex may be defined as innately determined behavior that 15 controlled by stimulation of a specific group of receptors and takes the form of a predictable set of muscular contractions. Instunctive behavior, on the other hand, is more than a complicatron of reflexes, although of course it involves reflex elements. It is not always controlled by a specific stumulation, and may in fact be aroused by a perceptual lack ("reaction to defictt," Lashley, 1938a; "vacuum activity," Lorenz, cited by Tinbergen, 1942). Instunctuve hehavior does not consist of a predetermined sequence of muscular contractions, and yet it has a constant and predictable end result. The spider of a given species will spin a web bighly spectic in design, though the movements necessary to produce it vary with the distance of the objects to which it is attached, the knd of movements and number of trips made by a bird in building a nest (in those cases where the design can be foretold from the burd's species) depends on the knd of ma- 


\section{The Relation of Irstinct to Learning}

terial used and the shape and size of the crotch or ledge that supports it, and so on.

All this means that the behavior, though it cannot be called reflex, is still continually under sensary influence, more or less direct. At each stage in the construction the muscular activity varies with circumstances and is such as to produce a certain perceptual effect. This indurect sensory control is demonstrated whenever accident destroys part of the structure The muscular activity then does not contunue un a predetermined sequence, but reverts to an earlier stage, in such a way as to restare the missing part Since the behavior is continually responsive to such events, it must be under afferent influence throughout.

Now we have already śeen that the $\mathrm{A} / \mathrm{S}$ ratio, or proportion of association-area cortex to sensory cortex, elfects the durectness of sensory control over behavior and the promptness with which it can be establushed. In order to make this relationshup meaningful for lower forms in whoh the cerebral cortex is absent or insignificant, let me speak here of the proportion of internuncial to afferent cells (assocsation/sensory cortex being only a partucular case of this more general relation) A massive afferent system and a negligibly small internuncial one implies an Immediate and direct sensory control of behavior, and one in which any particular sensory constellation would tend to have a single effect on behavior. The larger the internuncial system, the more slowly can a sensory control be set up A sensory contral that is establushed only with expenence, however, means that there is an element of learoing in the subsequent behavior-and so an increased variability Thus, with phylogenesis, the predictabulity of instinctive behavior would steaddly decrease as the ratoo of afferent to internuncial tissues becomes smaller

This does not of course explan why the instinctive behavior of any one species takes the particular form it does, but at this point we do have an explanation for the fact that instunct is most endent in lower species, in which internuncial structures are small, both absolutely and relative to afferent ones. Before specfic instuncts can be explaned, much more must be known about the bebavior in question and the finer nervous structures that deternine it it is a reasonable expectation that the knows- 
edge will be obtained, and that the explanations when they are found may be much simpler than one would suppose

To show that the expectation is not chimencal, that this does not simply dodge a crucial difficulty for the present theory of behavior, let me cite the earler discussion of the "spatal hypothesis" or position habit. The position habit of course is not called instinctive by psychologists, presumably because it only shows up durng the course of learning, and learning and instunct are supposed to be incompatble But it is so completely predictable in the rat that it might well be called instinctive. Before a means was found for analyzing the behavor (Hebb, 1938a, 1938b, Hebb and Willams, 1941) it almost appeared that the rat had a thoroughly mysterious capacity for perceivng compass direction. It turns out, however, that his behavior is simply response to the most constant stomulation of his environment (pp 91-5).

Eventually, explanation at a smular level can be expected for other species-predictable-or instunctive-behavior It is almost certain that the mysteriousness of the processes determining $\mathrm{m}$ stinct is generally overrated, when analysis has finally succeeded, the process turns out to be quite different from what it appeared at first (Schnerla, 1948) The nest-bulding of the pregnant rat may be the simple result of a disturbed temperature control (Kinder, 1927) The visual preference of the honeybee for complex flower forms appears to come from the fact that these forms produce a greater flicker frequency in the bee's eye (Wolf and Zerrahn-Wolf, 1937) and thus have a maximal stimulating value. The preference is not at all an mnately determined perception of a particular pattern as distinct from other patterns There is still an innate factor, but a much simpler one than it seemed at first to be, and the evidence does not require the assumption that some special and complex process is involved in the behavior Another example is the apparently purposeful behavior of the newly hatched loggerhead turtle in going directly to the sea (Daniel and Smith, 1947), which, however, is phototropically determined

I have already suggested that "instinct" may fundamentally involve some learning even at lower phylogenetic levels, if the kund of learning is strictly limited and if it may occur in a matter 


\section{The Relation of Instinct to Learning}

of seconds (pp. 113, 115). This is quite speculatve at present, but one thing is clear, at least, that there is no endence to justify the a priori assumption that the control of learned and the control of instinctive behavior are quite separate processes (Beach, 1937, 1939). In mammahan behavior, certanly, we cannot separate out one group of responses that are innate, others that are learned Constitution (the factor of heredity) may dominate in determining some properties of behavior, experience (the factor of learning) in others, but there must be a gradation from the one type to the other

In the same way, mstinct is not a separate process from intelligence or insight It is intelligence, or insight, that is innately limited in variety. An animal low in the scale must see a particular situation in a particular way because of the overwhelming mastery of afferent over internuncial activity, whereas a higher animal with a more variable association-area activity, under imperfect sensory control, may see it in a number of ways and respond accordingly. now to one property of the stimulation, now to another But the mechanism of response is not different in the two cases, except in complexty

This should be more explicit in one respect It has been argued that expectancy is an essential explanation of motor vanability in mammalian behavior At the same time, it must be clear that instinctive behavior does not involve an expectancy of its remote biological results (this would be the worst sort of teleology)

I have said that instinct and intelligence differ in complexty of mechanism, and one of these differences is in the degree of anticipatory central action that is called expectancy, and in the temporal extent that expectancy can span At the lowest levels of instunctive behavior there can be no expectancy whatever, where the internuncial activity is completely under sensory control, without intermission At intermeduate levels, there may be an expectancy of the immediate consequences of the single act in an instinctive sequence (the burd may have an anticipatory concept of a twig placed in a certan part of the nest, before it is actually land there, the rat whose pups have been scattered may possibly retrieve them under the influence of an expectancy of perceiving them in the nest again). Even this degree of 
expectancy, however, involves learning, expectancy must be an association between a present state of affairs and one that has followed that state of affaurs (or similar ones) in the past. The temporal span of expectancy in mstnctuve behavior must vary, from zero to the human expectancy of pregnancy and birth nine months after copulation (and the difficulties of rearing a child for twenty years after that) To the extent that expectancy occurs it must represent an influence of learning, not an inborn knowledge of the future. 


\section{Problems of Motivation: Pain and Hunger}

This chapter and the next aim at a broader picture of the problem of motivation than is usually drawn when neural mechanisms are to be discussed The biologically primitive hunger, pain, and sex drives are important in behavior, but these are not all of motivation. In particular, there is an essential problem in play, and in the changes of motivation that occur when bodily condition (mcluding humoral factors), and the environment in all important respects, seem unchanged

In the present chapter, motvation is dealt with first as a theoretical conception, particularly in relation to the theory of learning; we can then try to see how the more general ideas that result from this exammation are to be applied to the specific problems of pain and hunger The following chapter will be an attempt to round out the picture by treating the spontaneous variations of motivation that appear in such different aspects of behavior as play and sleep.

\section{THE CONCEPT OF MOTIVATION}

The term motivation concerns two distinct questions. (1) why is an anmal active at all, and (2) why does the activity take a parbcular form? Ostensibly, (1) is the fundamental question Actually, it is (2) that modern psychology is usually concerned with-though the writer who uses the term often seems to be talking about (1).

We have seen (Chapter 1) that early experiments with nervemuscle preparations made it easy to think of the nervous system as mert, unless sturred up by some outside irntant. It then 


\section{Problems of Motivation Pain and Hunger}

seemed necessary to look to sensory stumulation for the cause of any muscular contraction Now, however, it is known that the central nervous system is continuously active, and the necessity has disappeared, as long as we do not ask about the patterning, the tuming, of muscular contractions

In fact, most of the musculature itself is continuously active (for whatever reason), and the chief problem the psychologist is concerned with, when he speaks of motivation, is not an arousal of activity but its patterning and direction Little time is spent discussing the source of an animal's tonic contractions and random movements, in the first stage of a learning exper1ment. What we have usually wanted to know is why the random ، movements change and become directed The man question for psychology concerns the timing of muscular contractions, not therr arousal

There seems still to be some confusion on this point Psychological theory sometimes seems to be stull looking for some extraneural irritant to account for maternal behavior, for example. In an earlier day, it was thought that maternal behavior must be aroused by the sensory stmulation of distended breasts, male sexual behavior by distention of the seminal vesicles, the search for food or water by stomach contractions or dryness of the throat, and so on. Without exception, these ideas have broken down Desire to drink can occur when the throat is moist or anesthetızed, eatung continues after the stomach has been surgically removed This point has been adequately discussed by Morgan (1943) The older manner of thinking persisted, however. As contradictions accumulated, a tendency appeared instead to postulate "central receptors" instead of peripheral ones as motivating agents By this device, it is still possible to discuss motivation as a stimulation that arouses activity of a particular kand *

- The imphed distinction between theories of mòtivation does not merely concern use of the word "stumulation" It is more than a question of deflmition The sex hormones, for example, are known to have a primary action on the central nervous system This action might be exther (1) by exciting specific cells-in which case it would be meaningful to speak of a "central stumulus" -or (2) by changing the excitability or frequency prop- 


\section{The Concept of Motivation}

It has frequently been proposed that all behavior, and all learning as well, must be motivated The proposition may be taken in either of two ways First, we can treat it as a fundamental assumption We then infer the existence of a motivation, known un no other way, when an apparently unmotuvated act is seen (When the rat reaches food and, before eatng, goes off to some other part of the maze, he must have an exploratory drive or motivation. He has a hoarding motivation when he gathers pellets of food without eating them.) Such a use of assumption is often legitumate, what it really does is define motavaton or drive, and such definitions have been an essental part of the scientufic method But here the term motivation becomes so broad, when all the deviations of behavior are considered, as to have little usefulness, except as a convenient reference to the fact that behavior in general is directed and organized The fate of the term becomes that of insight when all learning is sard to be insightful.

Or, secondly, one can define motivation independently, as an extraneural bodily condition or an urrtant that acts on specific neural cells The proposition that all behavior and learming are motivated then becomes untrue, on the existing evidence, and with it the proposition that learning is directed only by motvation.

And yet there is an umportant problem here. In its simplest terms, the question that has bothered psychologists is this An animal learning to solve a problem makes some nght movements, some wrong. What is it that remforces the right ones and discourages the wrong? Why are the right ones remembered in later trials, the rest forgotten? This has been a persistently bafflung problem, and its solution is essential to the theory of behavior.

The difficulty may seem trinal If so, however, the reader may be begging the question by sayng to himself, in a commonsense (and animistc) way, that the animal of course can see that one of his movements has had the right effect, others not. ' But if the question is asked, What neural processes constitute the

erties of a system of cells, without being able to induce ectivity in the system directly. If the term "strnulus" is to have a determinate mearing, it should not perhaps be overextended to include the second reference (2). 


\section{Problems of Motivation. Pain and Hunger}

"seeng"? the solution is evidently as far off as ever. The sumplicity of the question of reinforeement, or selective retention of some responses and not others, is like the simplicity of the fundamental postulates of mathematics that are taking centuries to ravel out A sumple common-sense answer has no value whatever, the first thing one has to do 15 get away from the animism that saturates common sense. Having done that, there remains an uncommonly difficult scientific problem.

What it involves will perhaps be seen best in showng its relationship to Pavlov's theory of conditioned reflexes Pavlov's farlure to solve the problem is one of the decisive reasons why psychologists have had to abandon or change the theory.

\section{THE THEORY OF CONDITIONED AEFLEXES}

Pavlov (1927, 1928) formulated a simple rule for the occurrence of learning Any stumulus that acts repeatedly at the same tume as a response will form a connection between the cortical cells involved. Subsequently, the stimulus will be suffcrent to arouse the response. (The classic example is a boll rung at feeding tme. after a number of trals, nnging the bell alone makes the animal's mouth water) The origunal or uncondtoned stumulus (food in the mouth) has an innate relationship to the response. The substutute or condutioned stimulus (the sound of the bell) has an acquired relationship. The relationship is supposed to be set up in connections from seasory to motor area in the cortex.

This assumption is the keystone of the theory The theory as a whole is of course much more elaborate, with a number of other assumptions, but I have not oversimplified the point under discussion An afferent actinty at one point in the cortex, and an efferent activity at another point, at the same tume or immodiately after the afferent one, is all that is needed (with enough repetitions) to establish a senson-motor connection, and all behavior is determined by such connections, mnate or conditioned

This means that all acts at the tume of a particular stimulation would be associated with it; and the act that has most frequently occurred would be most strongly associated. Now in problemsolving, or any learning situation in which a number of acts are 
possible but only one rewarded with food, "mistakes" occur frequently Often, in the first trials of maze learning, the same mistake is repeated much more frequently than the "correct" behavior. Yet the net effect is that mistakes vanish, and leave only the correct responses Instead of being associated, the act that occurred frequently is forgotten. Its elimination, also, can hardly be ascribed to an mhibition Pavlov defined inhibition as producing a cessation of activity and leading drectly to sleep But repeated mistakes do not tend to make the animal drowsy or motionless They are often followed by an increased activity.

Maze learning, however, is not a very clearcut instance. For the theorist, a more crucial argument has been found with Pavlov's own procedure, and minor vanations of it. This argument is in the fact that the conditoned stimulus does not evoke the unconditioned response, as Pavlov ampled (Zener, 1937, Hilgard and Marquis, 1940)

The conditioned response is not a duplicate of the uncondrtioned, but something new All the elements of the uncondrtroned response may be there, but if so some of them are minmal, and the total pattem is significantly changed. When the dog hears the bell, he does not usually make eating movements, with his nose in the food dish, untal the food itself appears. Conditioned and unconditioned stimuli, then, are not just two ways of arousing the same combmation of effectors.

If the objection is made that no one would expect the dog to chew and swallow untl food shows up, the answer is that Pavlov's theory says he will. The conditioned stimulus is supposed to set up connections wrth any following effector actioity; and eating movements follow the conditioned stumulus as much as the secretion of saliva does. If the critic of this argument points out that a dog is too intelligent to respond to a bell in such a way, be is really critcizing the original theory A separate intelligence, to tell the dog when his conditioned reflexes may operate, would be a deus ex machine. One may suppose that the dog is intelligent, but not that his intelligence is separate from his neural activity, and we are examining the theory that all higher neural activity consists only of conditroned reflexes."

- The phystological and clinical literature suggests that a number of workers still feel that Pavlov's conclusions are a factual statement of what 


\section{Problems of Motivation: Pain and Hunger}

\section{MOTIVATION IN CONDITIONED-REFLEX THEORY}

Now let us see whether some principle of motivation can be added to the theory and thus make it able to account for the facts Hull (1943) has made this addition explicitly to show why "right" responses become conditioned, others not. Hull proposes that those acts that are immediately followed by a "need reduction" are retained, and he believes that with this change Pavlov's main structure can stand, successfully reducing behavior to a set of conditioned reflexes. That is, Hull has introduced motivation as a supplementary prnnciple The idea was used earlier by Thorndike, who called it the law of effect.

The law of effect is roughly this An act that is closely followed by satisfaction, pleasure, or comfort will be learned, one that is followed by discomfort, forgotten or elmminated Partly because of the result of subsequent experiments, Thorndike's (1931) later treatment is not in terms of pleasure and pam but refers to a very vaguely defined condition of "belonging" as the factor that makes for learning. The response that will be re-

is known to go on in the cerebrum It is important to see the difference between the facts he demonstrated and the theory based on the facts Pavlov has deservedly had a great influence on psychology, and his theory has not been rejected because it is too physiological but because it does not agree with experiment Apparently, Pavlov isolated himself from the contemporary literature, and his theory took no account of psychological discussions after 1900 Cntucism at present is based on later experiments, physsological as well as psychological

The man points can be hsted briefly (1) Sumultaneous sensory and motor actuvity is not enough to establish a condinoned reflex when the motor activity is directly aroused by stimulation of motor cortex (Loucks, 1935, I938) (2) The data published by Pavlov do not agree with his theory of a slow urradiation of excitztion across the cortex (Loucks), and the strychninization method reveals a knd of cortical organzation quite unluke what Pavlov assumed (Hilgard and Marquus, 1940) (3) Irradiaton, by which Pavlov accounted for stmulus generalization, does not provide for generalvzation of stunulus intensity (Hovland, 1937, Hilgard and Marquis), and the theory also has considerable trouble with the knd of pattern generalization lllustrated in figure 1 (4) Finally, it does not seem possible to translate Pavlov's system into terms of problem-solving or serial learning without senious modiflcation. This is the point discussed in the text. 


\section{Motivation in CR Theory}

membered and repeated is one that "belongs" in the total stuaton. Unfortunately for the older and simpler law, punishment by electric shock for the right response may help the subject to learn as much as punishing wrong ones Mild punishment for either may be better than no punishment, whether with animal or human subjects (Muenzinger, 1934, Drew, 1938, McGeoch, 1942). It is therefore not possible to say that discomfort always acts to elıminate a response. The vast literature on this issue, and the facts that led Thorndike along with many others to abandon the original law of effect, have been surveyed by Post$\operatorname{man}(1947)$.

To substitute belongingness for comfort and discomfort makes the law of effect extremely vague and says nothing about physiological processes. The theory presented here can be regarded as an attempt to fill in the gap left by Thorndike's treatment of this point. In psychological approach his ideas are in many ways identical with those that were outlined earlier (Chapter $\theta$ ). They constitute, that is, a theory of association, as distunct from a theory of conditioned responses, in which an association may be between autonomous central processes instead of between afferent and efferent processes

Hull (1943) has attempted to deal with the problem in another way, essentally by going back to the earher law of effect without the vagueness of Thornduke's later formulation. Unfortunately, he has done this by retaining the idea of motivation as stumulation, and of all learning as consistng fundamentally of sensori-motor connections.

"Effect" becomes a reduction of need, and a need is defined as follows. "When any of the commodities or conditions necessary for individual or species survival are lacking, or when they deviate matenally from the optrmum, a state of promary need is sard to exast" (Hull, 1943, p 17). Further, the need affects behavior by stmulating special receptors, etther peripheral or in the brain itself (Hull actually starts out by saying that they "probably" do [p 18] but thereafter takes the point for granted, his argument and diagrams deal with stimulations of particular hypothetical cells )

What the theory says in short is this All behavior is determined by sensory stumulations All learning consists of form- 


\section{Problems of Motivation: Pain and Hunger}

ing or strengthenng S-R connections An S-R connection is strengthened whenever the stimulus, $S$, and the response, $R$, occur together and some need strmulation, $N$, decreases at the same trme The S-R connections amount to conditioned reflexes. What Hull has done, in this modification of Pavlov's ideas, is to postulate a special class of stmulus that (1) must be present before learning can occur, and (2) must then decline The declne, and the decline only, determines the occurrence of new connections No way' is suggested as to how the decrease of strmulation promotes the formation of connections

To Hull's system as a whole vigorous objections have been made by other psychologists Its weakest point, and clearest departure from the facts, is in the treatment of motivation as biological need.

In the first place, the apparently clear and precise definition of a need will not hold water A need occurs "when any of the commodities or conditions for individual or species survival are missing" One of these needs is "the occasional presence and specrahzed recrprocal behavior of a mate." But the absence of a mate cannot excite sensory receptors. By this definition, evidently, another need is for testes in the male anumal and simultaneously ovaries and uterus in the female. Species survival is not possible otherwise But lack of testes is not usually a motsvating condition, nor can it excite receptors The mjection of male hormone would not affect the needs of the animal, as defined, since he is still incapable of fertiluzing a female and contributing to survival of the species Yet his behavior sexually is that of a normal animal once the injection has been made

The preciseness with which "need" has been defined is illusory It is a biological statement that does not coincide with the psychological conception Hull had in mind when he wrote First, then, it must appear that Hull's reformulation of the law of effect is not the precise postulate that it was meant to be A system of deductions that makes repeated use of it may have a fundamental flaw.

Secondly, the law of reinforcement, as it is now called, is open to the same critcism that made Thorndike abandon the law of effect in its earher form As we have seen, pain does not always act to elimmate a response Incidental learning, unaccompanied 
by reward or the avoidance of punishment, does occur According to the theory, the rat in the maze should learn nothing about it until one of his responses is accompanied by a decrease of hunger or thirst, or escape from electric shock, or some similar reward In actual fact, when he is allowed to run in the maze without reward or punishment the rat learns a good deal about it. This is shown by the small number of errors he makes later, when he is put into the maze hungry and allowed to find food there (Blodgett, 1929, cited by Hilgard and Marquis, 1940)

The third point is the extreme narrowness of the range within which needs as Hull has defined them must operate This is a return to the old idea that the only sources of action are such things as hunger, thirst, pain and concupiscence, with motherlove thrown in for good measure. Stimulation that is frequently associated with one of these is supposed to acquire the same kind of influence It is not denred, therefore, that man or animal may act without prospect of pnmary reward But according to the theory these secondary motivations can be set up only by repeated concidence with a prmary one, and the connection between the two must be frequently reinforced thereafter

Emphasis on biological needs seens to limit animal motivation much too narrowly It excludes conditions that clearly mfluence behavior and learning (Allport, 1946, p. 342), and it may have the unfortunate effect of preventing the student who takes the hypothesis seriously from seeng many of the facts of behavior For example, chldren spontaneously avoid dark places even though no unpleasant event has been associated with darkness (Chapter 10) They can learn what to do to make their parents come into the bedroom and turn on the light But the "need" here is for more stmulation, not less It is the theoretical preoccupation with hunger, thirst, sex, and avoidance of pain, as sources of motrvation, that has led many child psychologists to deny that there is any emotional response aroused primarly by perceptual lack, such as darkness. Nevertheless, the evidence is strong that such responses occur.

The point has been labored enough As far as one can see at present, it is unsatsfactory to equate motivation with biological need Theory bult on this base has a definiteness that is very attractive, but it may have been obtained at too great cost. To 


\section{Problems of Motivation Pain and Hunger}

follow this line seems to requre etther a persistent disregard of facts such as those referred to above or the continual addition of new assumptions as soon as one gets beyond the subject matter of the classical experiments on rote learning in animals

It is clear of course that the primitive drives of paun, hunger, and sex are often of overwhelming umportance. We need an approach to motivation that nether minimizes these things nor fals to provide for the unrewarded learning that also occurs when the anmal's belly is full and his sex drive satiated.

\section{AN ALTERNATIVE TREATMENT OF MOTIVATION}

The alternative treatment of mothvation that is required by the present theory is here introduced in general terms and is made more precise in the following discussion of pain and hunger This will still have some of the essental points of conditionng theory as developed onigmally by Pavlov and later by Hull The differentiation of two stages or levels of learning (first, the assembly of cerebral cells, and second the establishment of connections between assemblies) makes it possible, perhaps, to treat motivation in a Pavlovian way without inserting a spectral postulate in the theoretical structure Pavlor necessarily thought in terms of a sensori-motor condiboning, but, with the data avaulable from electrophysiology of the central nervous system, one can conceive of conditioning as the establishment of relations between cerebral neurons (in the first place) and between systems of neurons (in the second) The result, as I have suggested, is a theory of associations, from another point of new, it is a way of incorporating some of the advantages of assocration theory into a theory of conditioning-a common ground perhaps for Thornduke (1931) or Woodworth (1938), with Hull (1943) or Guthrie (1946)

Learning according to the present ideas consists of a lasting change of faciltations between the activities of specific neural structures. The change results when two structures (single patbways or assemblies) that have sufficient anatomical connections are active at the same tume The first learning establishes an organization of individual transmission units into an assembly. But later leaming, the knd that is usually studied 


\section{Problems of Motivation Pain and Hunger}

follow this line seems to requre etther a persistent disregard of facts such as those referred to above or the continual addition of new assumptions as soon as one gets beyond the subject matter of the classical experiments on rote learning in animals

It is clear of course that the primitive drives of paun, hunger, and sex are often of overwhelming umportance. We need an approach to motivation that nether minimizes these things nor fals to provide for the unrewarded learning that also occurs when the anmal's belly is full and his sex drive satiated.

\section{AN ALTERNATIVE TREATMENT OF MOTIVATION}

The alternative treatment of mothvation that is required by the present theory is here introduced in general terms and is made more precise in the following discussion of pain and hunger This will still have some of the essental points of conditionng theory as developed onigmally by Pavlov and later by Hull The differentiation of two stages or levels of learning (first, the assembly of cerebral cells, and second the establishment of connections between assemblies) makes it possible, perhaps, to treat motivation in a Pavlovian way without inserting a spectral postulate in the theoretical structure Pavlor necessarily thought in terms of a sensori-motor condiboning, but, with the data avaulable from electrophysiology of the central nervous system, one can conceive of conditioning as the establishment of relations between cerebral neurons (in the first place) and between systems of neurons (in the second) The result, as I have suggested, is a theory of associations, from another point of new, it is a way of incorporating some of the advantages of assocration theory into a theory of conditioning-a common ground perhaps for Thornduke (1931) or Woodworth (1938), with Hull (1943) or Guthrie (1946)

Learning according to the present ideas consists of a lasting change of faciltations between the activities of specific neural structures. The change results when two structures (single patbways or assemblies) that have sufficient anatomical connections are active at the same tume The first learning establishes an organization of individual transmission units into an assembly. But later leaming, the knd that is usually studied 
expermentally, is a change of faclitation between organized systems, a changed interaction between one complex of assembly actions and another. In the termmology of Chapters 5 and 6, learnung is a change of relationship between cortical phase sequences-or conceptual activitses-because of their having occurred together.

It then follows that the experimenter who wants an anmal to learn some particular thing must acheve some control of the phase sequence In a sugle envronment, the phase sequence may be extremely variable Puttung an animal into a particular situation does not determme what particular conceptual activity will result. Therefore, it cannot determine that a faclutation will be establushed between the conceptual activities the experzmenter is unterested in When the expermmenter takes further steps to limit the vanety of conceptual activity that will occur In an anumal, he sets up a motivation The term motivation then refers (1) to the existence of an organized phase sequence, (2) to its durection or content, and (3) to its persistence in a given direction, or stability of content.

Thus defuntion means that "motivation" is not a distunctuve process, but a reference in another context to the same processes to which "msight" refers, it also means that the wakng, normal adult anumal always has some motrvation (because of items 1 and 2 in the definition) though xts persistence in any one direction (3) may not be great.

If a bodily need is found to limit the variability of central neural action, it can be used experimentally to control learning, but this of course is quite dufferent from saying that the anmal will learn nothing unless he has first been deprived of food or mjured by electric shock. Without such means of control one is less able to predict what will be learned in a given situation, but one would still expect some learning to occur

Now we have to ask how such bodily conditions can control conceptual actuvities. Furst, parn.

\section{PAIN}

The relationshup of pain to leaming has already been taken for granted, in the assumption that certain afferent processes act 


\section{Problems of Motivation: Pain and Hunger}

primarily to disrupt central neural activities (p 150). Disruptive processes are a general classification that includes more than pain. In most sensory modes there is an intensity limen at which avoidance appears Below this point, the stumulation may be sought out-that is, it is "pleasant", above it, the same kind of stumulation produces avoidance and, if the avordance is unsuccessful, behavioral disturbance. With different kinds of stimulation, the avoidance limen falls in a dufferent part of the intensity range Pain is not unque, but may seem so partly because there is a great range of intensity above the hmen of initial avordance Pain, therefore, as a particular class of event, is regarded here as a disturbance ongunating in the somesthetic afferent system, though its mechanism may be like that of disturbance origunating from certann sounds, smells, or tastes

It should be understood that we are not manly concerned here with the conscious experience of pain, and consequently that the class of event discussed is not quite coterminous with pan as discussed in the introspective literature. There is, for example, what has been called "pleasant pain," which will not be discussed apart from pointing out that the phenomenon may be exactly parallel to "thrill" or pleasant fear ( $p$ 233).

I class pain then as a disruptive somesthetic event The disruptive action is usually limited, and is mainly at higher levels in the bram stem or in the cortex At the spinal level, tissue injury may arouse only integrated reflex responses (Sherrington, 1906). At the thalamo-cortical level, the disturbance must still be focalized, since of course pain is referred to specific parts of the body. Its effects may be widespread, but if the disturbance becomes really general it is emotion, not pain.

With these assumptions, it has been shown how pain might control learning by disrupting one phase sequence, leaving another unmolested. Essentially, this is a limitaton of conceptual processes in a given environment Pain then is a means of channeling motivation, as motivation was defined in the preceding section It is not a source of learning, from this point of view, but limits and direets it However, pan does have an energizing effect on behavior, and in some crrcumstances it appears to facilitate learning directly. To this possibulity I sha]l return.

Now let us see what the assumptions mean concerning pam 
itself. There have been in general two theories of pain (Morgan, 1943). In one, pain is not a special sensory mode but an effect of overstumulation of receptors for heat, cold, or pressure In the other, pain is a sensory mode with its special receptors, peripheral nerve fibers, and conduction paths in the spinal cord. Each of these theones has its difficulties, each has something to recommend it The two seem to have been thought of as mutually mcompatible, and exhaustive if one is proved wrong, the other must be right But following up a suggestion made by Nafe (1934), and makng a companatively small change in each theory, we can find a way of combinung them The combination has the best features of both, and covers certain facts that may be equally destructive of etther theory singly

Against the first, perrpheral-intensity theory, is the existence of pain spots in the skin. here a weak stumulation can arouse pain, although a much stronger stimulation elsewhere does not. Pam seems also to depend masnly on the activity of $C$ fibers in the sensory nerve This in itself demes that intensity is all that matters in pain, but, even more, intensity in neural terms must be a matter of impulses per second, and $C$ fibers are the very ones in which conduction is slowest and which deliver the fewest number of impulses per second to any one point in the nervous system

However, there are strong arguments against the alternative, sensory-mode theory If pain is a sensory mode, it is known that its receptors must be free nerve endings, and free nerve endings are also known to mediate pressure There may of course be two kands of free nerve ending, histologically indistingushable However, very weak or very brief strmulation of pain spots or pain fibers produces no pain, as Nafe has pointed out, this has obliged supporters of the sensory-mode theory to hold that there may bo "nonpainful pain."

Also, it is not at all clear that pain can be permanently abolished by clean surgical section of one or two of the tracts only,

- And see Weddel, Sinclair, and Feindel (1948), whose views in some respects are identical with those elaborated here Wortis, Stem, and Jolluffe (1942) have also anticipated some of this treatment They suggested that the faster-running umpulses in $A$ fibers can prevent $C$ umpulses from traversing certain synapses, so preventing pain. 


\section{Problems of Motivation. Pain and Hunger}

in each side of the spinal cord there are tracts that are manly involved in the production of pain, but are they solely involved in it? Finally, there is no specialized cortical area for pain as there is for touch, and apparently no specialized pain nucler in the diencephalon or lower brain stem if specialized tracts for the conduction of pain do exist, what do they conduct to, other than the centers of somesthesis?

The suggestion of Nafe, to which I have already referred, is that pain somehow concerns the patterning of central activities aroused by the pain stimulus, and with this it seems that the mam contentions of both classical theories can be combined Let us forget peripheral intensity, as the essential determunant of pain, and think instead of a central intensity that could, as we shall see in a moment, be initiated by a weak stmulus in the periphery as well as by a strong one Let us also suppose that the pain fibers do not mevitably mediate pain, that the slow impulses, and the central connections of these fibers, are such that they are peculiarly effective in producing sudden massive discharges in somesthetic nucler at thalamic or cortical level, with a widespread effect This would be pain Isolated stumulation of a pain spot then would not necessarily produce pain but would usually do so.

It is a highly significant fact that pain is often the result of conditions that must decrease sensory activity, mcluding activity in the pain fibers or their central connections. As Lashley (1938c) has observed, pain is often an accompaniment of injury to the somesthetic system all the way from peripheral nerve to thalamus In some cases, the pam may be due to irritation, but in many cases there is little basis for the assumption, If the facts can be explained without it As the work of Adrian (1934) and Weiss (1941a) has shown, an essential function of normal sensation is a regulation of central firing, without control, the firng is spasmodic or with a local hypersynchrony, with it, the cells do not buld up to the point of spontaneous discharge but are fired diffusely in a frequently varying pattern If higher somesthetic nucles are deafferented, everything we know about neural function indicates that the cells must eventually fire, and fire more or less in synchrony, with massive jolts to other nucles connected with them. When it is supposed that 
pain may be a disruptive activity at the cortical level, it must be supposed also that a conceivable source of pain is a decrease or absence of peripheral sensory activity, even in pain fibers

This hypothesis does not specify what kind of disturbance constitutes pain Evidently there are devations from normal sensory function, including hypofunctions, that never mvolve pain, so the hypothesis is certainly not complete As for pain in healthy persons, the predominantly slow impulses in the sensory nerve may produce pain only (1) because of some peculiarity in the pattern of thalamic activity they arouse, apart from total frequency of impulses in a given part of the afferent tract $(\imath e$., apart from intensity at this level) Alternatively, pain may occur (2) because the slow impulses are pecularly effective in arousing excessive bursts of fing from the thalamus Either explanation will suit my more general thess, though the second is the one I have preferred But the essential point is this a theory of pain must provide for the fact that an increase of pain often results from hypofunction in afferent structures

I have already referred to Lashley's observation, that pain may follow lesions anywhere in the somesthetic system up to the thalamic level. In Head's (1920) classic experment on the regeneration of cutaneous nerve, hyperalgesia was promment According to Bonng (1916) the hyperalgesia is not a lowenng of the pain threshold. Thresholds, mcluding that for pain, are raised, yet the pain when it does occur may be of extreme severity Wolf and Hardy (1947, p. 173) make the same pount, concerming patients with thalamic lesions It seems, obvious that the rise of threshold means a decrease in the total amount of afferent activity The pain that is so often felt in the phantom limb (Riddoch, 1941) also seems an obvious accompaniment of a decreased sensory activity After amputation, regenerating nerves may form neuromas that are a source of sensory iritation But it is very unlikely that the total of this afferent activity can be as great as would result from the use of the whole limb, and why should pam fibers in the neuroma be more irrtable than in the normal limb? Neuralgia, finally, is usually thought of as an irntation, as a hyperfunction that must be reduced by deadening the nerve But only too often an injection of the nerve fails to work-just as might be expected if the pain, in such cases, is 


\section{Problems of Motivation. Pain and Hunger}

essentually related to a hypofunction. The original source of the neuralgia must have been a pathological process in the nerve or the nuclei to which it leads It is highly improbable that a pathological process can increase the efficiency of any fiber, even a pain fiber, is it not more likely that total afferent activity has decreased, and that a disruption of central tuming has resulted from an incomplete peripheral control of central activity?

Return for a moment to the older theories of pain. The evidence concerning hypofunction directly denies the theory of peripheral intensity. It also makes difficulty for the sensorymode theory, but a more clearcut argument against the latter is found in the fact that the pattern or combination of stumuli $2 s$ often the decisive factor for the occurrence of pain. Stimulation of $A$ produces pain, of $A$ and $B$ together, no pain. The clearest example has been provided by Head (1920). Stumulating the th of the glans penis with water at $45^{\circ} \mathrm{C}$ is painful, but the pain totally disappears when the glans is immersed more deeply in the water Such facts might be accounted for if pam impulses are "inhibited" by other sensory impulses, but this explanation has really grave difficulties

The difficulties of the inhibition idea are clearest if we consider an organ, such as a sound tooth, that contains pain fibers but has never been the site of pain. We can hardly suppose that there are normal nerve fibers that have never once been active over an indivdual's lifetime If we suppose that they are active, but at a low level that is not recognized as pan, then we have conceded that the activity of the pain fiber does not essentially meduate pain, and the alternative is to suppose that an inhibitory action of other fibers has throughout a lifetime prevented the occurrence of pain It is very doubtful that an inhibitory process can be so uniformly and contunuously efficient (p. 212).

It seems in short that the pain receptors in a sound tooth, or in the cornea of an eye that has had no gnit in it for months, must have some other function-with weak or brief stimulations, that they are pressure receptors as well. It is still reasonable to think of them as pain receptors if, as it seems, the thalamus innately has a very low tolerance for the actuvity they inituate, if their action tends innately to result in a disruption of any behavior that is accompanied by tissue damage. 
It may be objected that pain is a unique sensory experience, and therefore that it must have its special receptors and central connections which serve no other function. But competent introspective observation has always found more than one knd of pain experience, and Titchener (1920) gives four prick, clear pain, quxck pan, and ache, each no more closely related to

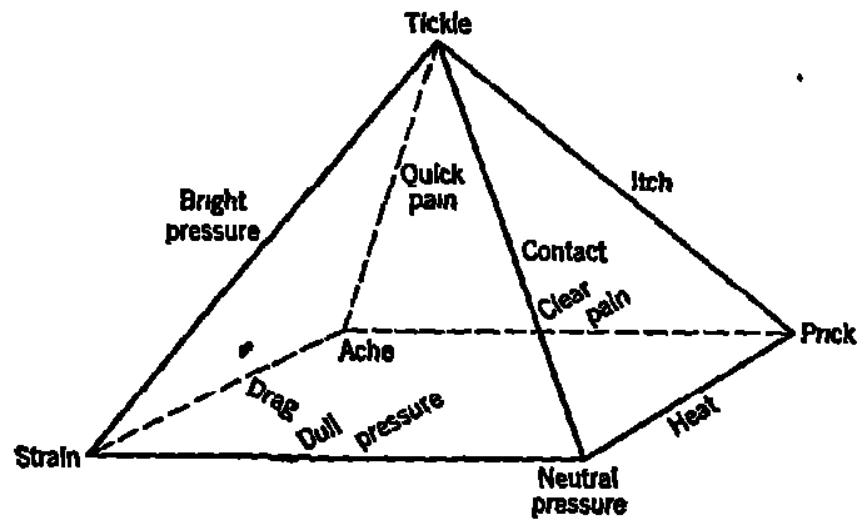

Figuke 18 Diagram from Titchener, 1020, showing the independence of vanous kands of pain, untrospectively determined By permission of the American Journal of Psychology, Karl M Dellenbach, Editor

another accordung to his diagram (figure 18) than quick pain is to bnght pressure or to ttch." Clearly, the subjective evidence in itself does not establish a separate modality of pain, internally unfied, and distinctive from others. How then have we arrived at this impression of uniqueness?

I have already suggested that pain may seem unique because the limen of avoidance in the intensity range of the pain

- Professor Dallenbach, in a personal communication, has pointed out that other evidence may render thrs account of pain, by Titchener, out of date. I cite It here, however, as showing that competent introspectuve observation does not inevitably find pain to be a single entity, whatever the conclusion from other experiments may be Professor Dallenbach has drawn my attention also to his ingenious experiments with Burns, Stone, and Edes (Dallenbach, K M, "Pan history and present status," Amer J Poychol, 1939, 52, 331-347), which controvert the theory that pain is intensive stumulation of tactual receptors only, but do not appear sufficient to show that it is a separate and coequal sensory mode 


\section{Problems of Motivation. Pain and Hunger}

stimulus is so low It may also seem unque because the characteristic event causing it, damage to body tussue, is not immeduately reversible. Once the damage is done the stimulation of nerve contunues and avordance does not put an end to it. It is common knowledge that there are nowses that are almost unbearable, although the fact ts hard to demonstrate in the laboratory (Miller, 1947). But one can move away from the nouse or cover the ears, at once decreasing the inteasity of stimulation These conditions must tend to determine a special kund of learning related to pain, with a more strongly marked avoudance and a greater emotional element, even though the mechanism of the disturbance when it does occur is the same in different sensory systems.

Let me say again that, this hypothesis of pain is incomplete. Hypofunction often occurs without paun, syringomyelia, a dogenerative disease of the cord, provides an outstanding example. The hypothesis is of value in suggesting how pain may result from hypofunction, and in reconciling divergent vews of normal pain. Intensity is still a factor, but at the cortical or thalamic level, and the arousal of $\mathbf{C}$ fibers may still have a special sensory quale, not disruptive of behavior at the very lowest level of intensity or duration, but promptly becoming disruptive unless they are part of a much more extensive stumulation that has a thoroughly well-organized central action This central organjzation would be a duffuse firing, and would make impossible whatever pattern of local synchronization it is, in the ventral nucleus of the thalamus, that leads to local disruption in the cortical somesthetic area and so constitutes pam

It may be proposed that the optumal condition for the arousal of pain without gross bodily unjury is a punctate stumulation of the relatuvely inefficient $C$ fibers-being inefficient, they do not readily set up a well-organized central action, and, accordingly, that it is not a complete hypofunction of afferent systems that conduces toward pain, but a decreased efficiency. Higher somesthetic nucler then lack therr normal degree of sensory control and become hyper-responsive, and yet are stall exposed to an intermittent "triggering" from peripheral stimulation A complete deafferenting consequently would fully eliminate pain, whle pathological changes without complete destruction, up to and per- 
haps including the nucleus ventralis thalam, would increase it

Specifically,-lesions of perspheral nerve would then be considered to have reduced the efficiency of $A$ fibers to that of $C$ fibers-that is, make them into pain fibers, higher lesions would have a sumlar effect by reducing the efficiency of $A$-fiber control of thalamic and cortical tissue It would thus be concenvable also that "quick pan" could be aroused by undamaged $A$ fibers, though less readily, sunce their control is more efficient than that of the $C$ fiber

It is possible now to complete the picture of pain as related to learning, and especially as having a positive value in learning The psychological entity pain must be a disruptive action at the cortical level, but the pain stumulus has other effects In the spinal animal, it may arouse only a well-coordmated reflex avoudance (Sherrington, 1906). In the diencephalic animal, it is likely to produce sham rage, with an integrated action of the whole organism (Bard, 1934) It has been seen in the preceding paragraphs that the stumulus that by itself arouses pain may have no such effect when combined with other stumulation The pain stumulus arouses pain when its central action is unorganized and interferes with other, organized action, but not when it enters into and forms part of an organzed neural action.

Pavlov (1928) and Liddell (1944) have shown that the disturbing effects of cutting and burning the dog's skn disappear completely when the pain stumulus is regularly followed by feeding, in the conditioned-reflex procedure The transformation from typical pain responses to feeding responses was gradual, once it was established, there was immediate relapse when the site of pain stimulation was changed to new parts of the body.

This means that there were synaptic changes in the cortical region in which the focal pain disturbance ongmally occurred, leading to the disappearance of pain from one region of the body, not from others. In that one region, the pain stumulation now aroused organized cortcal activity. Thus conclusion is meaningful for the observation of Muenzinger (1934) and Drew (1938) that giving a rat electric shock for the correct response (followed of course by food) moreases the rate of learning. In Drew's experiments, when the food itself was electrfied so that the rat got a shock with every bite, eatung was voracious. In 


\section{Problems of Motivation Pain and Hunger}

such,a case, once the central effects of the pain strmulus bave become organuzed, we must regard the stumulus as essentially motivating. just as a blow of the why may be motivating and energizing to a racehorse that appears already to be exertung himself to the utmost-a further stumulation that adds to the effectiveness of a pre-exstent cerebral control of behavior

\section{HUNGER}

The treatment by Morgan (1943) is taken here as a basis for discussing the problem of hunger, with two questions to be kept in mind how the approach to motivation outlmed in thes chapter accords with the expermental facts of hunger, and whether it can be of any further value for understanding the behavior involved

The fundamental problem is to know what controls the mitzation and termination of eating (or drinking) Hunger is defined here as the tendency to eat, conscious awareness or verbal report of hunger is important only as a convenient index of that tendency. Eatung is not necessarily dependent on the occurrence of stomach contractions, since the surgical removal or denervation of the stomach does not prevent or decrease it It is not necessaruly dependent on blood-sugar level, since hunger is reported when the blood-sugar level has not changed (Scott, Scott, and Luckhardt, 1938) Both stomach contractions and blood sugar are related to hunger, but in no sumple way

The termination of eating also has no simple control The anumal that has been starved eats more than one that is well fed, but the termunation of eatung comes before the food can have much effect on boduly tussues A thirsty animal will drink approxmately the amount of his water defict, and stops drnking before the water has been absorbed. The experment can be done in fact with a dog with a fistula in his throat, so that little If any of the water is absorbed, yet "satiation" decurs (Bellows, 1939)

The problem is complicated but not fundamentally changed by the demonstration that there is not one but a number of hungers (Rrchter, Holt, and Barelare, 1938, Young, 1941). The rat lackng any one of a dozen or so substances will choose a 
diet contaning that substance, selectively correctung a spectic deficiency. We still have the same problem: what is it that initrates the eating of salt or sugar, or draking water, and what terminates it? Appetite and satzation are selective, but it is desirable if we can to avord postulatug fifteen or twenty mechanusms (allowing for future discovery of other hungers besudes those we already know), all in control of normal eating If possible, we must find a single mechanism to comprehend the facts.

The neural mechanism that determines such motivated behavior as eatung or sex activity Morgan has celled the "central motive state" This, for the theory presented in this book, evdently is a phase sequence or conceptual activity It does not zequire that we postulate a special process to deal with bunger, but only that the process already postulated have some distuncthe feature for each of the separate hungers Conceptual senes $A, B$, and $C$ might correspond to one hunger, $A, B$, and $D$ to another, and so on.

The total process would fit Morgan's specifications for the central motuve state, sunce (1) the phase sequence is independent of input from any specific sensory mode, (2) it has a central pruming action that reinforces some sensory stumulations and not others, and (3) it evokes specific forms of behavior, since each phase in the phase sequence may have its specific motor facilltation Morgan's final specification (4) is that the central motive state arouses general activity or restlessness for the present theory, this is qualified by saying that the restlessness must occur only when the environment fails to supply the specific stmulation that the central matrve state would reinforce-that is, when the hungry anmal is not exposed to food, or the sexually motivated anumal does not have a sex partner at hand.

This point is of some importance We must distinguish between an integrative, motwatung effect of a lack of food, and a disintegratrve one The integrative action appears in any purposeful search for tood, and in eatung That it occurs, in the expenenced anmal and in a famlinar environment, is an evident fact, it is the real fact with which most discussions of hunger have been concerned. But the picture will not be complete if we recognize only this aspect of the problem, and assume im- 
plicitly that the physiological need of food equals hunger (that is, the tendency to eat).

There are two reasons for denying such an assumption. One is that leaming is involved in hunger, as we shall see in a moment. The other is that, unts this learning has occursed, the lack of food is apt to be disruptive of behavior. The effect of the lack appears in restlessness, whob is not drected behovior but imphes an instability of durection. restlessness as such is not a purposeful seekng but activity without purpose. With more expenence, the restlessness becomes a durected search B10logically, it has served a purpose, having made a certain kind of learning possible, but this does not make it, from the psychological pount of new, purposeful-and the point here is that the first effect of a lack of food is not an moreased directedness in behavior (unless the mouth happens to be in actual contact with the nipple, when there is drectedness at the reflex level)

Even in experienced subjects the need of food has disintegrating effects. The relation of hunger to emotional disturbance is notorous, and Goodenough (1981) has shown it clearly in a study of anger in chuldren. Lack of food, or of some particular element in the diet, such as salt (Saphir, 1945), can produce profound and lasting emotional disturbances

Furthermore, hunger itself-the tendency to eat-may be disrupted by a lack of food Hunger practrcally disappears about the fourth day of starvation in man, and there may even be a "repugnance" to food (Carison, 1916) In the rat, the rate of eatung is markedly lowered by the fourth day, and the amount eaten zs also low (Bousfeld, 1935). Physical changes in the alrmentary canal would account for the smaller total intake, and even perhaps for a 50 per cent dechne ws the rate, but they would not account for a total loss of human appetite if the neural mechanism of hunger were undisturbed

In chronic starvation, however, the hunger mechanism seems undusturbed even though the degree of malnutrition may be great. Is it possible that the difference here is due to learnung, in the same way that learnugg changes the restlessness of the first need of food unto a drrected search? Let us look at some evidence canceraing the relation of learning to hanger, before 


\section{Hunger}

returning to the question of how a lack of food might dusorganize behavior.

It is common knowledge that a person eating on an uregular schedule when he is used to a regular one, and so without his usual environmental cues for eatung, may be "hongry without knowng $2 t^{n}$ With a deficiency sufficient to produce headache and irritability, he may find out what is wrong only when food tastes unusually good or when his symptoms disappear as a consequence of eating. The fact obviously suggests that eating is partly a leamed response to certain envronmental events. Hunger, defined as the excitation of a neural mechanism that controls eating, is not a simple direct product of a need for food. There are cognituve factors to be considered when hunger is studied in the mature anmal.

It does not affect the value of Ruchter's study of food preferences (Richter et al., 1938) to recognize that has method clearly permits of a short-term learaing as a factor in preference. Young (1944) and Beebe-Center, Coffin, and MacLeod "in fact bave shown that the learnung has a strong infuesce.

Also, it is well known that tame laboratory rats deprived of food, taken from their cages and offered food in a strange place, will for some time eat little or nothing. Before he will run in a maze for food reward, the rat must be given the rontme "prehminary training," extending over a period of days. Inanition, meanwhile, may become extreme. It has been generally supposed that the failure to eat is due to an undependent emotional dusturbance, but some preluminary experuments (to be reported in more detail elsewhere) suggest strongly that this is not the whole explanation.

Six rats were thoroughly babituated to an enclosed field, contaning a number of movable objects, over an 8-day period. Then they were deprived of food for 24 hours, and put into the field with food and water present. The amount of time each rat spent eating, in a pernod of 120 seconds, was recorded. The procedure was repeated a number of times as one would carry out a learning experiment, to see whether the amount eaten would be affected by "practice"-whether it would be small at

\footnotetext{
- Personal communicatoon from Dr J. G Beebe-Center
} 


\section{Problems of Motivation: Pain and Hunger}

first, and increase on subsequent days, whether "forgettung" would occur when practice was nterrupted, and whether changes in the environment might change the response, but without inducing emotional disturbance to account for failure to eat

The amount that a rat can eat in $\mathbf{1 2 0}$ seconds is not nearly enough to maintam health, but the average tume spent eating in the first test was only $\mathbf{7 9}$ seconds, one rat eating for as short a tume as 35 seconds. On subsequent days, the amount increased, dropped whenever practice was interrupted ( $2 e$, after the rats had been left for a few days in their cages with food and water present, before being tested again) and rose once more when practice was resumed, and dropped with each change in the arrangement of objects in the expenmental environment The changes induced exploration but no evident signs of emotion It is also important to note that there was no tendency to take a bite of food and then explore, or even to take a quick bite whenever exploration brought the animal near the food (this shows up later, as the rat becomes more sophistrcated).

A second expenment was done to check on these results, and also to see what would happen when exploration was elimunated. In this experiment 10 rats (from another colony) were tested mdividually in a living cage, being allowed food for 300 seconds after a 24-hour deprivation, and again after a 48-hour deprivathon. The average tume spent eating was agan low on the first test-41 seconds one anumal ate for as short a tume as 5 seconds On the second test, the average rose to 140 seconds But the total amount eaten was still estimated to be not more than onetenth of what would be necessary to mantain even a depleted body-werght.

It seems therefore that the rat must learn to eat the amount he needs, and "satiation"-cessation of eating when no external event has occurred to interrupt it-occurs before the physiological needs of the unsophisticated animal have been met.

These results agree with the indications that human hunger is partly dependent on being aroused by events associated with eatung They also show that a theory of hunger does not necessarily have to account for an automatic regulation of the amount 


\section{Metabolic Factors Disturbing Behavior}

eaten, that will just equal the amount needed. The termination of eating or drinking may be determined by experience, in part at least (but partly as the termination of other pleasurable actuvties is determined, by a knd of fatigue Chapter 9). This learming process is prolonged. I have the impression that the rat, offered food for short periods only, at 24-bour intervals, will take from 3 to 5 weeks before he gets to the point of promptly eating the amount of his food deficit as soon as food is made avalable.

Must there not be something of the same sort of learning in the hunger of the dog and cat? The expenment suggests that when hunger in the dog is to be studied it will be necessary to know the animal's history or, better, to rear the animal in the Jaboratory with a systematic control of his experience of the need of food. Data obtanned when this has not been done may make hunger appear more an mnately established process than it actually is.

\section{METABOLIC FACTORS DISTURBING BEHAVIOR}

The evidence discussed in the preceding section has indicated, first, that lack of food tends to disrupt behavior, producing restlessness, discomfort, urrtabulity, and, in the extreme degree, emotonal apathy Secondly, a learnung process seems to be unvolved in transforming this primitive disturbance into hunger as we know it in the adult animal or human being

We may now ask what the source of the ongunal disturbance is before considering (in the next section) the processes that transform it The present discussion, primanly of the origins of hunger, has a broader implication and will be relevant also to the problem of emotronal disturbance due to metabolic disorders.

It is well established that the need for food affects the central nervous system directly, through changes in the blood content (Morgan, 1943). (There may of course be pernpheral effects as well ) The effect of chemical changes in the blood content would influence the rate of firing of the nerve cell $A$ nutritive deficiency may be thought of as usually lowering the rate, though a calcum deficiency might raise it (Lehmann, 1937a; Bronk, 1939), the umportant thing being the change of rate 


\section{Problems of Motivation: Pain and Hunger}

rather than the drection of change It must also be assumed that any particular chemical infiuence would not affect all cells equally. There is a considerable variety in the make-up of central neural cells and their reaction to changes of blood content. This is shown in the selective susceptrbulty of particular parts of the central servous system to particular diseases and toxins; it is also shown in the dufferentual effects of anoxia and of vanous anesthesias. The firng of some cells may be especually changed by a lack of thiamin, others by hypochlorema, and stull others by hypoglycemia

Now it has been seen that neural integration is fundamentally a question of timing, quite apart from the particular theory of integration that has been developed in these pages. Metaboluc changes, by alterng tume relations in neural firmg, must tend to disrupt behavor-not merely slow it up, but disorganize it.

In the present theory, timing has its effect in the functioning of the cell-assembly and the interrelation of assemblies. diffuse, anatomically irregular structures that function brietly as closed systems, and do so only by virtue of the time relations in the firing of constituent cells Synaptic changes are necessary to the setting up of an assembly, but these act by coordinating the action of two or more cells The fing of one cell immediately after another is not determined by synaptic knobs alone but also by what is going on in some other cell or cells. Synaptic knobs alone cannot determine that a particular system will function as such

Furthermore, an individual cell or transmission unit may enter ioto more than one assembly, at different times. Which it will form part of, at any moment, depends on timing in other cells, and to enter into any assembly requires that its frequency accord with the time properties of the active system If a metabolic change affects one cell much more than other cells in the system, it must drop out, if enough cells drop out, the assembly does not function

If, instead, all the cells in an assembly are affected to about the same extent, the system would continue to function, but the facilitation delivered to other assemblies might be modified in a way that would disturb, or redurect, the phase sequence (that is, the sequence of assembly actions). 


\section{The Mechanism of Learning in Hunger}

At any one moment, the action of an assembly may be considered to be_on an all-or-none basis, the system functions as such, or it does not. But an affected assembly might function at one time and not at another, in agreement with the statustical approach of Chapter 4 Suppose for example that the functioning of an assembly requires the activity of $n$ pathways in parallel at some point in the system, and that $n+4$ are anatomically avarlable At any time, some pathways are refractory but there is normally a margin of 4 at this point, maintaining the operation of the system. If now a change of blood content lengthens the recovery cycle of some of these cells, the average number of refractory pathways might rise from 2 to 3 , say, and the upper lumit of the number from 4 to, $\theta$ When 5 or $\theta$ are refractory, the number of avalable pathways would fall to $n-1$ or $n-2$, and the assembly does not function for the moment. A half second later, it might again be responsive

One might assume that longer-established assemblies would have in general greater safety margins With the greater development of synaptzc knobs, due to more frequent activity, the number $n$ might decrease (p 66) This would imply that older habits, and longer-established memorres, would be most resistant to desruption by metabolic changes of blood content

In general terms, these ideas provide a rationale for the actually observed disturbance of behavior that may result from changes of blood content, whether in hunger or otherwise In hunger itself, the blood change might be a lack, as in hypoglycernia, or it might be the addition of the "hunger hormone" of Luckhardt and Carlson (1915) and Morgan (1943) This would be a substance liberated by depleted body tissues, with a selective effect on the finng of neural cells.

\section{THE MECHANISM OF LEARNING IN HUNGER}

The facts discussed earher, showing an element of learning in hunger, leave room at present for a considerable dufference of opinion as to how large a part it plays. In order to further define the problem for research, let us assume for the moment that the innate factor consists only of the reflexes of suckung, chewing, and swallowing, of spitting out noxaous substances, and 


\section{Problems of Motivation Pain and Hunger}

of the activities of the gastromtestmal tract-that all else is learned * The deficiencies of this approach will help us to discover what facts are needed for a better understanding.

Stimulation of the lips of the newborn infant elicits sucking, as a reflex activity. The same stmulation stops hunger contractions, and the effect is increased by the stumulation of taste buds and by swallowing (Carlson, 1916) In the adult at least the effect of stomach contractions is related to pain ("hunger is an uncomfortable pain sensation caused by stomach contractions" Carlson, p 97)-muld, but painful. In the infant, also, the behavior accompanying strong stomach contractions is in general not to be distingurshed from the effects of a loose safety-pin in the diaper.

Consequently, hunger contractions should have the same role as pain in their effect on learning As the contractions begin and get stronger, some tume after suckling, there would be an increasing disturbance of the intrinsic organization of activity in the infant's cerebrum (p 121) This organization it will be recalled makes for inactivity in behavior, other than reflex behavior, as it is broken up, an increasing restlessness should be expected. As a result, the moving mouth is likely to make contact with the nipple. Reflex sucking follows, with a further stimulation of taste buds and swallowing stomach contractions cease, and the restlessness disappears.

Later, as the infant becomes more mobile and explores more of his envronment, other things are investigated and enter the mouth, with simlar effects. Some things produce, perhaps, too strong a stumulation of salt, sour, or bitter receptors and are spit

- In correcting proof I realize how misleading this statement may be. Obviously, I assime that the "innate factor" also moludes all the mechanism that determines what kand of learning will take place It includes for example the susceptibility of cortical organzation to disturbance by hunger pangs or lowered blood nutnents

Throughout this book the reader may feel that the emphasis is too much on learming But this is only verbal Whenever one could think of several ways in which some particular learming might happen, but recognizes that the facts of anatorny or physrology allow only one of them, one is recognizang the exastence of the innate peculanties of the central nervous system AII mammalian behavior involves learaing, but the tonate factor is always there, determining what learning occurs and how 
out. Others fall below the avoidance limen, reduce to paste and are swallowed These edible substances, not only stumulating taste receptors but also being retained in the mouth and eventually swallowed, are evidently the ones that most effectively interrupt stomach contractions they provide more of the inhibstong stumulation, and more-prolonged stimulation.

The conditions necessary for learning are provided in this sequence of events As the sequence is repeated, the sensory stimulation-visual, olfactory, gustatory, and kenesthetic-organizes assemblies of cells with a motor facilitation of the various activities of eating This brings eatung farther and farther under cortical control, and so mcreases the possible influence of learning. The sight, smell, and taste of food, the tactual stimulation of mouth and throat, and kinesthesis from chewmg and swallowing are the stumulu that arouse this assembly action To a certan extent, the sensation from stomach contractions itself would become conditioned to arouse the same activity Though pnmarly disruptive, like pain, this sensation should like pain be capable also of being conditioned to eating responses. It has been seen that the potentially painful stimulation is not disruptive (that 1s, it is not pain) when it forms part of a stimulation that has well-organized central effects Finally, the development of conceptual processes controlling eating makes possible an association of eating with other conceptual processes

Schematic and oversimplified, this discussion still shows the way toward avoiding oversumplification It points out three ways in which hunger-an organuzed activity in the corticodiencephalic system-can be aroused (1) by the sight and smell of food and the sensation of eating itself, (2) by stomach contractions, and (3) conceptually, as the sequel to a train of thought. These agencies may summate One, on occasion, may not be effective by itself, but becomes effective when another is added to it Hunger can then be aroused or further mcreased by an attractive appearance of food, or by its smell and taste

Such effects are famliar, but since they do not fit into the rubric of bunger as a bodily need, or alternatively as sensations from the stomach, we are apt to forget them. Consider the salted-nut phenomenon Ordinarily, one can take salted nuts or let them alone-until one has eaten a mouthful, when it becomes 


\section{Problems of Motivation: Pain and Hunger}

much harder to let them alone. Hunger has mcreased but how? A lack of food cannot be increased by eating something, and stomach contractions are stopped by chewing and swallowing. If, however, we consider bunger to be neither a particular condition of the body, nor a set of sensations from the stomach, but an organized neural activity that can be aroused (like any other conceptual process) in several ways, the puzzle disappears The schematizing of the preceding paragraphs, then, has developed some of the further possibilities of Morgan's conception of the central motive state

The puzzle just referred to has been dealt with in the past by a distinction of hunger from appetite. ( ${ }^{\star A}$ taste of salted nuts does not mcrease hunger, it only increases appetite ") The solution is not a good one because no one has been very clear as to what appetite is, if it is not hunger. It seems in fact that hunger cannot exist without appetite; and "appetite" seems in practice to be a term that is applied to the tendency to eat when eating is not biologically desirable or valuable, "hunger" when it is desurable Such a distinction has no place in discussion of the physiological control of eating

If the explanation proposed in this section is nght, cuttung the vagus in the newborn infant will retard the normal development of hunger, by preventuag the postulated role of hunger pangs in the learning process Cutting the vagus does not stop eating in the adult, but it may have more effect at an earlier stage This would provide a drect experumental test of my schematyzing. It is not predicted, however, that no learning could then take place, since there is stull a mechanism of addiction to food to be discussed, which would also provide a basis for learning The explanation predicts that cutting the vagus would retard the development of hunger, perhaps sernously, but would not prevent it entirely

\section{HUNGER AS AN ADDICTION}

I have already discussed the metaboluc changes due to lack of food as a disturbing influence on behavior. The treatment of hunger can now be completed by considering the relation of these changes to the learning process a relation that makes eat- 
ing, in the mature animal, equivalent to an addiction, as Carlson (1916) long ago suggested.

It may be objected that hunger cannot be related to metabolic changes, because of the experument by Scott, Scott, and Luckhardt (1938) in which it was found that hunger can occur in human subjects with no change of blood-sugar level. This experiment is valuable, but in the light of present knowledge, and the discussion of the preceding pages, it needs two things to be complete:

1 In the first place, we need to know about other blood components besides glucose. Glucose is only one of a number of substances involved in hunger. If there are nue specific hungers besides those for oxygen and water (Morgan, 1943), there are nune substances whose concentration in the blood must be exammed before it can be concluded that hunger is not determined durectly by the blood content

2 In the second place, since we know also that there is an important element of learning in hunger, the experiment should be repeated with subjects in early infancy. Only then will it be possible to say that stomach contractions are not determined, orignally, by changes of blood content, or to conclude that the development of hunger is independent of such changes. The obvious experiment, dealing with both points $I$ and 2, is to determine the levels of thiamin, chlondes, and so on, in addition to the level of glucose, at the time when a need of food begins to disturb infant behavior or when the adult actually reports hunger.

Until such an experiment is done we can only speculate, but a little speculation may help to define the problem. Return to the learning process of the last section In general, the cellassemblies whose activity is hunger would be organized in a condition of muld deficiency We do not know whether this condition is enough to produce a lowering of blood nutrients, or secretion of the hunger hormone, but, if it is, observe that a mild deficency would be the optumal condition for the functioning of the assemblies in question It is not a low blood sugar, or low chlondes, that would be dusruptive of an assembly, but a change from the level at which the assembly was organzed If bunger is establushed orgunally in the presence of a slight 
ing, in the mature animal, equivalent to an addiction, as Carlson (1916) long ago suggested.

It may be objected that hunger cannot be related to metabolic changes, because of the experument by Scott, Scott, and Luckhardt (1938) in which it was found that hunger can occur in human subjects with no change of blood-sugar level. This experiment is valuable, but in the light of present knowledge, and the discussion of the preceding pages, it needs two things to be complete:

1 In the first place, we need to know about other blood components besides glucose. Glucose is only one of a number of substances involved in hunger. If there are nue specific hungers besides those for oxygen and water (Morgan, 1943), there are nune substances whose concentration in the blood must be exammed before it can be concluded that hunger is not determined durectly by the blood content

2 In the second place, since we know also that there is an important element of learning in hunger, the experiment should be repeated with subjects in early infancy. Only then will it be possible to say that stomach contractions are not determined, orignally, by changes of blood content, or to conclude that the development of hunger is independent of such changes. The obvious experiment, dealing with both points $I$ and 2, is to determine the levels of thiamin, chlondes, and so on, in addition to the level of glucose, at the time when a need of food begins to disturb infant behavior or when the adult actually reports hunger.

Until such an experiment is done we can only speculate, but a little speculation may help to define the problem. Return to the learning process of the last section In general, the cellassemblies whose activity is hunger would be organized in a condition of muld deficiency We do not know whether this condition is enough to produce a lowering of blood nutrients, or secretion of the hunger hormone, but, if it is, observe that a mild deficency would be the optumal condition for the functioning of the assemblies in question It is not a low blood sugar, or low chlondes, that would be dusruptive of an assembly, but a change from the level at which the assembly was organzed If bunger is establushed orgunally in the presence of a slight 


\section{Problems of Motivation. Pain and Hunger}

deficiency, the return of blood sugar and so on to normal would tend to distupt at, and equally, a more extreme lowering of the blood-sugar level This accounts for a loss of appetite in starvation, and is a first step toward accounting for satiation

Suppose that an expenenced, adult animal is exposed for the first time to a severe lack of food Both the increased hunger pangs and lowered blood nutrients contribute to a disturbance of cortical organization and so of behavior If the disintegration of the hunger mechanism itself has gone so far that there is indifference to the thought of food, or if the anumal put where he can find food makes no effort to seek it out-that is, if the hunger mechanism is not excitable conceptually - the sight and smell of food may be more effective, or food in the mouth may be. This sort of thing of course has been observed in starvation. The anumal is apathetic about food until he has eaten a mouthful, and then shows an moreased hunger (the "salted-nut phenomenon" agam) Once swallowed, the food is absorbed and reverses the slow fall of blood nutrients, mcreasing the possibulty of actual hunger as an organized cerebral action.

Now observe that each occurrence of such a sequence of events in chronic starvation would tend to set up new cellassemblies (or modify old ones) by the same process that set up assemblies in the normal infant. The new assemblies, however, would be such as to function best in a condition of low blood nutrients. The learoing then would establush a hunger that could occur strongly in the presence of starvation, and not as before only with a mmimal need of food

Hunger establushed in the presence of lowered blood nutrents, and having the effect (through eatung) of raising them, would be physiologically the equivalent of an addiction-biologically valuable, but still an addiction There has occasionally been confusion in distingushing between addiction and habit. Some writers apparently have seen no difference (except possibly in strength) between a habit of drunkang mulk at breakfast and a habit of drinkung coffee But an addiction mvolves more than a relationship of overt response to certain sensory stimulations otherwise Sanka, with the taste and smell of coffee but without its caffeme, would satisfy every need that coffee does and could be substituted for it without the victum's knowning that anything 
had happened. Unfortunately, it cannot the addict may not know what has happened, but he will know that something is wrong An addiction, therefore, besides involving habit, also acts so as to mantain a blood concentration of some substance that is necessary to stable neural functioning, or whose absence results in emotronal disturbance, restlessness, and discomfort or pain. This is a trite statement, but one of its implications should be considered What has happened to prevent integrated neural function, in the absence of the drug?

We know, first, that there is always a penod of "learnmg" or apprenticeship, in addiction, and we know also that an addiction lasts longer than the overt withdrawal symptoms of physiological disturbance. It may be proposed, therefore, that the learnung is a genume learning, and that it consists in part of the change of assembly action discussed above in relation to starvation. It follows that an addiction must be considered to be more than a metabohc reorganzzation. it uncludes a neural reorganzzation also, and one that tends to persist, since (as any tobacco addict or alcoholic knows) a "cure" is apt to bo unreliable and the addiction can be reinstated much more promptly than acquired for the first tume.

It is this very learning in addiction that we must know more about, to understand hunger. The learning can be described as an association between a sensory event and the subsequent euphoria being at peace with the world follows a full belly, or an injection of morphine The reference to "association," however, is misleading if it suggests that we understand the mechanusm involved We do not.

The problem of a learned preference, etther for a drug or a needed food substance, can be seen better in the light of Spragg's (1940) study of morphine addiction in chimpanzees. For example, bis experzment has demonstrated the difference between physiological dependence on a substance, and addiction to it. Daily injections established a dependence on morphune, demonstrated by withdrawal symptoms when the drug was withheld. But his chimpanzees still took a considerable time-from three weeks to three months-before they discovered the connection between (1) injection of the drug and (2) feeling better. Thus exemplufies an infermediate stage of addiction. Eventually, the 


\section{Problems of Motivation. Pain and Hunger}

chumpanzee in need of morphine tried hard to get an injection (by struggling to get to the accustomed room, getting the hypodermic needle and giving it to the expenmenter, and so on). The learning that leads to this final stage of addiction presents us with our problem, the same one that is involved in hunger." It has sometimes been sand that the rat in need of salt learns to eat it, by "experiencing its beneficial effects" Spragg has shown that an association of this kind can occur, even if its mechanusm is not at all clear, and his data should be kept in mund in dealmg with the problem of the self-selection of diet.

When a rat is suddenly put on a salt-free diet, he may be considered to be in the intermediate stage of addiction $\mathrm{H}_{2}$ behavior shows that he has a physiological dependence on salt, but he may still have to learn to look for salt-contaming foods and eat them selectuvely

The immediacy and reliability with which an anumal will correct a dietary deficiency seems to have been overestumated-at the least, overemphasized I have already cited Young (1944) and Beebe-Center (personal communication) as showing that the rat's preference for a needed food is not immediate, but learned With a low concentration of the needed substance, preference is establushed slowly, if may require as long as 7 days. With stronger concentrations, the preference appears early But showing that a rat already has a complete preference at the end of $2 A$ bours does not show that no learning has occurred We badly need a detalled study of what learning may occur in the first 10 munutes, or first hour, of a rat's exposure to a choice of foods.

The Editors of Nutrition Revew (1944) in a summary of the history of this topic, and a paper by Pugrim and Patton (1947), have drawn attention again to the unreliability of the selfselection method in many curcumstances The faulures must be kept in mind as well as the successes, if selective hungers are

- Dr Austan H Riesen has pointed out in a personal communication, that another exomple is the slowness with which arr crews learn what to do about axygen deficiencies, when oxygen is avalable

Obviously the law of effect would be the very thing to invole to account for such leaming, but I can still find no satisfactory way of fitung it into the present set of physiological conceptions 
to be explamed. Man is notoriously capable of malnutrition whle freely choosing his own det. This might be put down to bad habits, interfering with an innately sound mechanism of choice, but such an explanation would hardly account for hypochloremua, promptly reheved by taking common salt (Saphir, 1945), in men who had access to salt-shakers Their symptoms were severe, and their condition once more can be properly likened to that of Spragg's chmpanzees when they had withdrawal symptoms without seekng a morphine injection Man must often learn to take more salt when he moves to a tropical climate, and the learning is not autornatic.

Until such learning has occurred, it appears that the only drect effect of a need of some particular food, or of food in general, is restlessness, emotional disturbance, and malase. The lack of specificity in this disturbance may well be a clue to the nature of pathological eatung, arising from emotional disturbance, or of the alcoholic's tendency to eat a less and less adequate diet Clinically, there is an inescapable relation between drug addictions, food habits, and chronic emotional disturbance. The fact has some meaning on the assumption that the need of food or drug, and emotional dusorder, have a common element in a disruption of timing in the firing of central neural cells, and on the further assumption that this disruption is transformed into an organzzed action by a process of learning.

An hypothesis dealing with part of this learning process has been set forth in the present chapter, but there remains a part about which the hypothesis must be so vague as to have no present value Once more, Spragg's experiment makes the matter clearest He found it possible, once a full addiction was established, to abolish wsthdrawal symptoms by an injection of saline only-briefly, but nonetheless effectuvely (Spragg, 1940, pp. 95-96). Sumular effects are known in human addicts, and explamed" by a reference to suggestion. Here we have a sensory and perceptual event substtuted effectively for the physiological presence of a substance in the blood stream. The analogue in hunger is the occurrence of satiation long before a meal has been absorbed from the stomach and intestine. It has been argued that withdrawal symptoms, or the restlessness and discomfort of hunger, are the result of a disturbance of central 
206 Problems of Motivation. Pain and Hunger

organization If so, the sensory stimulation of seeng the hypodermic needle and feeling its jab, or the sensations of eating, must have some rather direct action in restorng the normal time relations in the firmg of central neural cells Some such possiblity has actually been envisaged in supposing that sensory processes may actively support and direct a given cerebral organizaton. Thus of course is in extremely general terms, and no details relevant to the present problem can be proposed. A more specfic hypothesis may be possible later, when some of the experimental evidence is avalable whose need has been shown in this chepter 


\section{The Problem of Motivational Drift}

The two primitive motivations of pain and hunger can be regarded as manly determuned by specuic boduly conditionstissue damage and lack of nournshment-although, as we saw in the preceding chapter, neither of these relationships is simple and nether is as yet really understood. Hunger, particularly, must be a very complex process.

A third motivation, biologically primitive, is that of sex. The sex drive is also ted to a definite physiological condition, the presence of certan hormones in the blood stream But it would once more be an oversimplification to make this the only factor. like eating, sex behavior is not reducible to any simple formula (Beach, 1947a), and it serves especially to make clear a further complexity of the problem of motivation This concerns the tume relations in the organism's responsiveness to a particular class of stumulation In sex behavior, the problem appears in the comparatively slow build-up of interest (that is, responsiveness to or seekng out of genital stmulation) and its frequently abrupt declime, following orgasm humoral conditions presumably remauning constant. A roughly parallel course of events is to be seen in other behavior, particularly in the alternation of sleep and waking, but also in almost any behavior that is a source of pleasure It should therefore have an important place in the theory of motivation

A constant property of mammalian behavior is a variability of responsiveness to the envronment that cannot be ascribed to conditions originating outside the nervous system-1t appears, that is, to reflect something intrinsic to the organization of cerebral activity. I have already emphasized in Chapter 7 this 
variability of attitude (or interest), and I propose now to look closer at its neural basis The reader will recall, further, that "motivation" has been treated not as a distunct process, separate from learning, insight, or attitude, but as a useful first approxlmation whose main reference is to the temporal organization of cerebral events (Chapter 8) The present discussion accordingly deals with the slow change or "drift" of motivation, but interprets motivation in a rather broad sense.

\section{THE PROBLEM OF A LASTING EXCITATION, FATIGUE, OR INHIBITION}

The problem of this chapter is the relationship between cychcal changes of responsiveness in the whole aninal and events in the nervous system A permanent change of responsiveness, of course, is called learning, and does not concern us here, but one that involves a contunung alternation of responsiveness to some stumulation, each phase lasting perhaps for hours before reverting predictably to the other, is a different matter In describing the behavior, such transient conditions may be called excitation or fatugue, which is a commonly accepted and useful terminology. It has, however, certain dangers and may conceal certain complications for the theory of behavior. "Excitation," "inhibition," and "fatigue," as prolonged neural conditions " determining the behavior of the whole an1mal, are conceptions which perhaps are not a primary concern for the neurophysiologist but which certanly need examination in psychological theory

When an animal is "excited" what happens-simply an increase of total cortical actuvity? What changes take place when excitability, or responsiveness to a particular kind of stumulation,

- It will be evident that a discussion of fatgue on this basis is not very closely related to the undertakng of Bartley and Chute (1947), who define fatigue as a form of experience that is the outoome of mental conflict They have not been mainly concerned with the neural mechanism of this conflict, or with the nature of the "loss of interest" which contributes to it Their emphasis on the complexities of fatigue, however, strengthens the present argument that it should not be lightly invoked as an explanatory conception. 


\section{Excitation, Fatigue, and Inhibition}

increases over a pernod of hours, days, or weeks? Is there simply a decrease of cortical firing in "mental fatigue"? When the cortex inhibits a certam hypothalamic pattern of response, is one group of cells preventing the firng of another group? Presumably, these questions cannot be definitely answered at present, but it should be seen that the answers are not obvious, and that, when the temporal characteristics of excitation are taken into account, the question of its neural basis becomes a crucial one, for physiological psychology at least And so with inhibition and fatigue

Consider excitation first There are two aspects of the problem. one is simply the duration of the excitation following brief stumulation. It seems generally recognuzed now that an excitaton which endures for minutes or longer at once rasses a question of the special mechansm mvolved. The all-or-none excitation in the nerve cell is almost infinitesinal in duration, bearing little relation to behavioral excitations that take an appreciable tme to develop The widespread theoretical interest in reverberatory or re-entrant pathways as the basis of a contunuing excitation in the central nervous-system shows that this side of the problem has been recognized (unluke the problem of a continuing inhibition, as we shall see in a moment).

But another aspect of the problem of excitation needs more attention A behavioral excitation, an increase in some boduly activity, is not necessarily a sign of an increased neural activity ether in the brain as a whole or in some one part of it The point is well llustrated by the process of getting drunk. A small amount of alcohol may be an excitant-socially, and in its immediate net effect on behavior-but this of course does not prove that alcohol is a neural excitant, it does not even prove that lower centers are being released from cortical nhibition.

There is a fallacy here which is most likely to be made when one is thinkng of different kinds of behavior as controlled by dufferent parts of the bram "There is an mcrease in certain behavior, therefore some part of the brain is more active, therefore-supposing that alcohol is not a neural excitant but a depressant-a higher mhibition has been removed."

Perhaps this is what happens The point, however, is that it is not logically inescapable A change of behavior does not 
necessarily mean that some neural units, active before, have become inactive, and that mactive ones have become active (except at the level of the final common path, and very often not even here since the same effectors may enter into dufferent responses) The same neural cells may stall be active, but in new combinations, when a different response occurs. This is one of the profoundly important implications of recognizing the role of tuming in neural transmission and of such conceptions as that of "optuonal transmission" (Lorente de No, 1939) Stumulate $A B$ and $C D$ and the excitation follows one set of pathways, stumulate $A C$ and $B D$ and the excitation may follow different efferent pathways and determme a totally different responsealthough the same internuncial cells are active

The behavior of the druak may' be produced because alcohol, depressing all neural cells, depresses some more rapidly than others and so changes the pattern of firng throughout the cerebrum The cortex of course may be more affected than the rest of the brain, but this is different from sayng that the actuvity of the brain stem actually increases with the administration of alcohol The idea that cortical function can be almost eliminated before lower centers are affected is scarcely tenable, and in fact the drunk, up to the moment of collapse, does not in any way resemble a decorticate preparation but continues to manfest behavior that we know is fully dependent on the cerebral cortex. The behavior changes and may be less adaptive, but is it less "cortical" and more "hypothalamic" or more "mesencephalic"?

The theory that drunken behavior is due to loss of cortacal inhibition is open to these objections first, it implies that the inhibition, during long periods of sobriety, has an astonishing duration and efficacy-a point to which we shall return, and secondly, it seems to imply that all drunks should act much more alike than they do, perhaps approaching the picture of sham rage before passing into stupor The other possibility, suggested above, is that alcohol from the first knocks out cells in the hypothalamus and striatum as well as in the cortex, and slows up firing rates, thus reducing the complexity of cerebral organization but leaving until the last some cortical as well as subcortical function Whether this is so or not, it is enough to 
show that there is no logical necessity of regarding drunken rudeness or hyperactivity as a loss of mhibition that permits an absolute increase of neural actuvity in some part of the brain

Two points have been made concerning a lasting excitation in behavior the long duration must be accounted for, and an increase of some kind of behavior does not always or necessarily mean an mcreased activity in some region of the central nervous system The same two points apply to inhubition and fatigue.

If a decrease of bodily activity that lasts more than seconds is to be explained by a neural mhibition or neural fatigue, some explanation is needed of the duration, and secondly, the decrease of bodily activity is not $a$ proori proof that such an inhibition or fatigue exists. There is nsk in assuming that any prolonged change in some aspect of behavior means a parallel change in some one part of the brain that mental fatigue is a neural fatgue, or that a suppression of anger means that some one set of cells (preferably cortical) suppresses the firng of some other set (preferably hypothalamic) A decrease of bodily activity does not always mean a decrease of neural activity. sleep may consist only of a change of the combinations in which cortical cells fire- $\imath e$, may essentially be hypersynchrony, with no decrease of total activity, and the EEG offers evidence that immobility and unresponsiveness can accompany a considerable cortical actuvity (Jasper, 1941).

It is commonplace to say that an organic whole has properties other than the sum of the properties of its parts in isolation The necessary corollary is this do not take for granted that the parts of a neural complex-or that any one part-must act in parallel with the action of the whole The fatigue that leads to prolonged sleep, or the disappearance of sexual responsiveness after ejaculation-often for hours afterward-1s not proof of fatigue in cortical cells, or of an mbibition of sexual centers, for corresponding periods of time.

Fatgue as demonstrated physiologically in the neuron (the refractory period) has a duration measured in milliseconds "Mental fatigue" may be a phenomenon of behavior, but its duration gives us no warrant for identifyng it with a primary neural fatigue. Similarly, the mhibition that is studied in lower neural centers (where a faurly direct inference is possible con- 
cerning what happens in the single neuron) is a brefly lastung process that is promptly followed by excitation. In a matter of seconds it becomes possible for any strong excitation to break through. Consequently, to speak of an inhibition that lasts indefintely is not just makng use of the established conception of spinal reflex mhibiton but introducing a different one. The conception may be quite valid, but it is not necessarily the cis of Sherrington (1925)

This point applies to the idea that pain fibers in a sound tooth can be contunuously prevented from finng (p. 186), that the sham-rage center in a good-natured, normal cat is contunuously mhibited by the cortex, or that a conditioned inhibition (Pavlov, 1928) can account for schizophrenic catatonia or for long-standing Freudian repressions Some of these ideas assume first that each form of behavior is determined by a separate part of the central nervous system, and all of them assume secondly that inhibition may have an extraordinary degree of persistence.

When for example damage to the frontal lobe is followed by an increased appetite, or increased sexuality, or increased responsiveness to carotid-sinus stumulation, some clmical writers are prone to take for granted that this is of necessity evidence that the frontal lobe normally inhibits whatever lower centers are involved Not at all, the normal action of the frontal lobe (and of the rest of the cortex) may be only to stimulate lower centers, but determining at the same time a particular timing of action in those centers The behavior that results is a function of this timing, removal of the strmulation (not inhibition) from the frontal lobe means a different pattern of firing and so a change in behavior. It must be realized that when a patient says nasty things to his frends after lobotomy he uses the same effectors as when he is polite-but in a dufferent firng combinabon, and the appearance of rudeness following brain damage does not show that there is a rudeness center in the brain, normally inhibited by the region that has now been damaged.

Decerebrate ngidity, again, may imply either (1) that the cerebrum acts primarily to dimmish the activity of ventral-horn cells, or (2) that its action stimulates only, and in stmulating continually modifies the activity of the intrnsic circuits of the spinal cord which if left to themselves would simply rase the 
level of tonic contraction. It is legitumate (though possibly confusing) to speak of the cerebrum here as "inhibitung" the action of these circuits, my point is to draw attention to the difference in meaning between such usage, and the usage when one speaks of mhibition as the suppression, by one cell, of activity in another. I have argued that the physiological evdence does not justrfy the idea that pain fibers, for example, may be indefinitely inhibited. It is a long step of inference from the prolonged inhibitory action of one complex set of neurons upon another to an inhibition of the individual cell

As a last example of this problem, consider the "suppressor areas" of the cerebral cortex (von Bonin, Garol, and McCulloch, 1944; McCulloch, 1944a, 1944b)

These areas, in or near areas 2, 4, 8, 19, and 24 of Brodmann, are widely spaced in the cerebral cortex and appear superficially to have a primary inhibitung action Stumulation of one of them in the anesthetzzed animal may have two effects relaxation of the musculature on the opposite side of the body, and disappearance of electrical potentials throughout the rest of the cortex This might appear to be a direct suppression of neural activity, as a positive action by one set of cells in the cortex that prevents the activity of other cells It might appear to justify, once and for all, the conception of a primary unbibitory function of the cerebral cortex.

The experimental evidence, certainly, has made it clear that stumulation of a suppressor area may produce a decrement of actuvity. But this leaves two questions: is this inhibition, in the sense that the vagus inhibits heart muscle, the same inhibition that presumably must operate in Lloyd's two-neuron spinal reflex arc (Fulton, 1943) $P$ and does it occur as a result of the normal action of suppressor-area cells, or only as a function of massive nonphysiological stimulation by electrical current or strychninization?

Suppose for a moment that the normal action of the suppressor area is a facultation of other cortical activity, that the function of areas $4 s, 19$, and so on, is like the function of the reticular substance in its faciltation of oculo-motor reflexes (Lorente de No, 1939) On the lines of the theory of the preceding chapters, integrated behavior requires a diffuse finng in the cortex. But 


\section{The Problem of Motivational Drift}

a massive electrical stimulation would fire all the cells in one region together and prevent their entering into the duffuse patterns of firmg necessary to the normal cortical control of behavior That is, the "inhibition" would be a loss of essential facilitation only.

The evdence presented by McCulloch (1944b) fits into such a picture Suppressor areas are drectly connected with the caudate nucleus, and direct stumulation of the caudate produces "large, long voltages" in thalamic nucler McCulloch, accordmgly, has suggested that the action of the caudate, in " suppression," is not a positive inhibition but the induction of rhythms of thalamic activity that are incompatible with normal thalamocortical reverberation

Finally, it is important to see that the suppression cannot be a direct inhibitory action from one part of the cortex on another, both because the strychnnuzed suppressor area does not appear to fire directly to other areas in the cortex and because of the observed time relations. The first effect of suppressor-area stimulaton is the appearance of spikes in the immediate neighborhood, but with no simultaneous loss of potentrals elsewhere Next, a spreading extznction of potentals moves outward from the point stimulated, affecting the suppressor area before affecting others. If the cells in this area were mhibitory, a high level of firing here should coincide in time with a low level elsewhere, but this is not what happens The suppressor area suppresses itself first, and then, after it has itself become mactive, makes others inactive. Obvously, this does not fit in with the idea that suppressor-area cells are mhibiting other cells but does fit in with the idea of a slowly spreading loss of facilitation-the implication of McCulloch's remarks

It is still early for final interpretation of much of the work that has been done in this field The nature of even spinal inhib1tion is still a disputed question, and there are serious dangers in applying the conception directly to the complexities of cerebral function, especially when the tume properties of a decrement of activity are found to be markedly different If it were safe to assume a true long-term inhibition (or, equally, fatigue) in cerebral action, it would greatly simplify the task of the psychologist. As matters stand, however, this is not justified. It is 


\section{The Time Course of Sexual Motivation}

necessary to recognize the problems for behavioral theory that result from_this fact.

Now let us see more specifically what some of these problems are.

\section{THE TIME COURSE OF SEXUAL MOTIVATION}

What we are interested in here, essentally, is the nature of a behavioral excitation, and this perhaps would present no particular problem if it were not for the way in which excitability and excitation develop and declme. The difficulty is clearest in sexual behavior.

The review of studies of mammalian sex behavior by Beach (1947a, 1947b, 1948) makes for emphasis on an interaction of sensory and central factors It has been clearly shown that the arousal of sex behavior does not depend on any particular sensory stimulation-on olfactory, visual, or auditory stımul, nor on somesthetic stmulation from the genitalia or from other regions of the body If gonadal hormones are also considered to be stimul, arousing receptor organs in the bram, even this stimulation is not always essentral to sexual arousal (smce gonadectomy frequently does not put an end to copulation) Nevertheless, all these factors normally play a part in the arousal. Ther effect is additive.

The presence of gonadal hormones in the blood stream does not often produce contunuous sexual excitement, and it appears that a normal level of hormone faclitates certain effects of sensory stimulation, selectively, rather than itself producing any specific pattern of coordinated responses When essentral stumulation is lackng, the motor pattern of copulation is apt to deviate from the norm or not to be completed Also, "There is evdence that the cortex is involved in the arousal and maintenance of the male's sexual excitement, and reduction in susceptrbility to sexual arousal subsequent to cortical mjury appears to be proportional to the amount of neopallum removed" (Beach, 1942, $p$ 187). The evidence thus gives us a picture of sexual excitation as being produced by the close collaboration of sensory and central mechanisms.

During courtship and during copulation up to the point of 
orgasm, activity in the "central excitatory mechanism" (Beach, 1942) summates, cooperatung closely with sensory stimulation and producing forms of behavior which mcrease that stimulation, until the joint effect of sensation and central facilitation reaches a final limen of response. Then, abruptly, the central excitatory mechanism is somehow discharged, or, alternatively, an inhibition or fatigue not evident before suddenly assumes maximal effectiveness and may last without interruption for hours.

It is, I believe, the first of the alternatives-a slow growth of excitation followed by a condenserlike discharge-that should be explored theoretically. It has already been said that it would be very desurable for psychological theory to be able to postulate an inhibition or fatigue in such cases to explain the transient disappearance of responsiveness But there are difficulties about doing so, as we have seen, and for the present there is no advantage in postulating a special inhbition in sexual behavior-as If we understood the excitation but not its absence Really, we do not understand either, and a transient disappearance of excitability may be inherent in the nature of the behavioral excitation itself Nothing that has been sayd in this discussion has made it impossible to suppose that the inhibition referred to above does exist, but if this is assumed, the essential problem of the time relations remains. The inhibition must be such as to last for days, on occasion, waning slowly in the older subject who may be capable of copulation only after days of recuperation from an earler copulation (Kinsey, Pomeroy, and Martin, 1948). This is no easier to explan than an equally slow rise of excitability.

Let us turn now to the similar problem of sleep, before asking how this problem might be solved

\section{THE ALTERNATION OF SLEEP AND WAKING}

The comparatively well-defined course of sexual arousal provides a clear example of a generally unsolved problem concerning cyclical changes of excitability. It cannot be asserted that fluctuations in sexual excitability, and the diumal fluctuation of general excitability called the sleep cycle, must have the same 
mechanism, but the two have important simularities, and it may be profitable to proceed on the working hypothesis that the problems are in essence the same The course of sexual motivaton suggests that a cyclical change may be mherent in the process of excitation, so that an extended period of arousal must eventually be followed by an extended period of unresponsiveness (though the reason is hard to see), and this of course is exactly what happens in the alternation of sleep and wakng

Physiological study of sleep has centered about two questions. (1) whether sleep may be determmed by one of several conditons external to the nervous system, such as muscular fatıgue, the accumulation of toxins, and so on, (2) whether it may be due to the action of a sleep center, or maction of a wakung center, within the nervous system itself (Kleitman, 1939, Nauta, 1946). Research has emphaszzed the umportance of a wakung center in the caudal hypothalamus, its action evidently has much to do with regulation of the sleep cycle Further knowledge on these two points must be obtained by physiological methods, and the present discussion will not contribute to it

But there is also (3) a psychological factor of "interest" or "boredom" in sleep (Kleitman, 1939) With thus we are directly concened The theory developed in the preceding chapters has definite implications about sleep, sleep has in fact been referred to repeatedly because it seems not to be an isolated phenomenon but an aspect of processes fundamentally involved in learning, for example, or voltion or emotional disturbance-and these inplications make it possible to deal with the "cortical" or "higher" components in sleep

Return to the earlier discussions of spontaneous neural activity, and its relation to sensation and consciousness (pp 8, 121-4, 146). We have, to start with, (I) the suggestion by Bartley and Bushop (1933) and Adnan and Matthews (1934) that unconsciousness may be the result of a lack of afferent activity, and (2) that this excitation normally has the function of breakng up the synchronous spontaneous finng of central nervous tissue. Add to this (3) that the EEG in sleep shows a marked hypersynchrony and (4) the general conclusion of Jasper (1941) that hypersynchrony may be opposed to adaptive behavior. We then have (5) a picture of sleep as a state in which sensation has lost 


\section{The Problem of Motivational Drift}

its usual control of central neural firing, and in which a large segment of the cerebrum has dnfted, from the diffuse firng necessary to adaptive behavior, into the hypersynchrony of unconsciousness.

From such ideas it is a short step to thinkng of sleep as a functional deafferentation of the cerebral cortex (Kleitman, 1939). But this phrase should be used cautously It may suggest for example that consciousness is a function of the cortex alone, or that the cortex is an isolable unit physiologically.

Even in the anesthetized anmal, cortical action is closely related to activity in cortico-diencephalic circuits (Morison and Dempsey, 1943, McCulloch, 1944b, Murphy and Gellhorn, 1945). There is probably a continuous interaction with the striatum as well (McCulloch, 1944b) Also, the term deafferentation may overemphasize the role of sensation in the sleep cycle of the normal anmal. Important though it $\mathrm{is}$, the level of sensory activity does not alone determine sleep or waking. This of course 1s the point made by Kleitman's "wakefulness of choice" as well as by hus emphasis on boredom as a source of sleep Wakefulness of choice is mainly a characteristic of higher animals. when a point is reached in the cycle at which sleep may occur, whether it does or does not depends on certam cerebral activities (referred to as conscious in man), as well as on the subject's environment.

With some qualification, however, the idea of a functional deafferentation is valuable How would it occur? Structural connections with the sense organs are, presumably, unchanged as one goes to sleep. The wakng center in the posterior hypothalamus, whose destruction leads to a contunuing sleep, is not the portal by which afferent excitation reaches the cortex. It cannot therefore simply act like a valve, periodically cuttung off the cortex from stumulation. However, it could be an essental link in a system "prining" the cortex for the reception of afferent impulses (Morgan, 1943) This would umply that the diffuse firing of the cortex cannot be maintained by afferent impulses alone; in terms of the present theory, it implies ether that one cell-assembly cannot arouse the next, in a "phase sequence," without supporting faclutation from the wakng system, , or else that some significantly large fraction of assemblies incor- 


\section{The Alternation of Sleep and Waking}

porate cells from the wakang system Damage to ths system, or synchrony of firng in it, would then interfere with assembly action and consciousness

This does not localize consciousness or the wakng state but assumes that the kind of activity throughout the cerebrum which we call consciousness is dependent on activity within a more limited region' primanly, the wakng center in the mammillary bodies, or in the neighboring posterior hypothalamus and anterior mesencephalon (Nauta, 1946), and the intralaminar and midline nucles of the thalamus (Penfield and Jasper, 1946, Jasper and Fortuyn, 1946). The essentral structures in wakng may also include the anterior cingular region (Brodmann's area 24, one of the suppressor areas) The cingular area is connected with the wakmg center via the mammullothalamic tract and the antenor nuclei of the thalamus, and Smith (1945) showed that stimulation of the area produces a transient condition apparently equal to sleep, except in duration

At first glance, this would mean that the cingular area is a sleep center, not a wakng center, but the function of a waking center is to maintain a diffuse finng, and a strong electrical stmulation finng a large number of cells in the area all at once would prevent it from having this function-the same point that was made in discussing the action of other suppressor areas

If thus the activity of the posterior hypothalamus is necessary to the maintenance of diffuse firng, the antenor hypothalamus may have a similar function, a pacemaker for the synchronous cortical firng of sleep It may, that is, be a sleep center, as Nauta (1946) appears to have demonstrated experimentally I shall urge in a moment that sleep is not a negative thing, not a mere lack of the organized activity of consciousness but the presence of an alternative organization, and the existence of a sleep center fits into this conception

From this point of view, we might consider that the cerebrum deafferents itself to produce sleep by falling to provide the faclitation that is necessary for environmental stimulation to maintain any control of cerebral firng The cerebrum also deafferents itself indirectly by ceasing to initiate motor activity, which at once means a decrease in sensory stumulation But we must go farther than this The conditions that produce sleep 
are not merely negative the fact is that some stimulations contribute positively to sleep, and sleep is reinforced by learning. Physiologically, also, it is evident that hypersynchrony is not a random affarr but organized, so that going to sleep means the positive substitution of one organization for another. This was referred to in an earlier chapter as the intrinsic organization of cerebral action, and it was suggested there how a prenatal learning process might contribute to it, if the organization is not simply due to the action of mherited pacemakers in the cerebrum (footnote, pp. 121-2).

On the same assumptions that justified the conception of the formation of cell-assemblies (Chapter 4), an afferent activity that coincides frequently with a synchronous firing will establsh synaptic knobs between the afferent fibers and any members of the synchronously finng pool with which they come into contact Repetstion of that afferent excitation will then tend to support the synchrony, or re-establish it, which means a mechanism of learning to go to sleep One may have to learn to sleep easily in the daylight or in unusual conditions, an habitual posture often helps one to get to sleep, and even habitual noises may do so, the lack of familiar stimulation delaying the onset of sleep or-if it occurs in the night-waknng the sleeper. Kleitman (1939) considers that the sleep cycle of the adult is a function of learnung, and in old age, when other learned behavior is detenorating, we find the cycle also tending to return to a more infantile stage Thus an accustomed stimulation may actively support hypersynchrony, as a learned relationship that is affected (just as other learning may be) by cortical atrophy All this involves a further qualification of the "deafferentation" 1dea, one might suggest perhaps that sleep is more a de-efferentation. The rationale of the de-efferentation should perhaps be made explicit here It is simply that normal waking behavior is a highly selective set of contractions of muscle, in which it is usually quite essential that antagonists are not both at peak activity simultaneousiy, but in alternation, and in which a particular contraction must have a particular strength and endure only for a particular length of tme. It is this that requires a dispersed fing in the cerebral cortex I have referred to Jasper's conclusion that hypersynchrony per se is opposed to adap- 
tive behavior, it is evidently opposed also to the process of thinking, which tends to break up even the moderate synchronization of the alpha rhythm Similarly, Kleitman has remarked the negative correlation between (1) hypersynchrony in sleep and (2) the responsiveness of the sleeper or the extent to which his dreams are organized and rememberable Finally, Loucks (1938) found that a conditioned response to direct stimulation of the visual cortex could not be elicited in the sleepung anumal. here, clearly, deafferentation of the cortex is not an explanation of the fallure of stimulation to elicit a response *

These facts appear to mean that the pyramidal-tract control of ventral-horn cells and cells of the motor nuclei of the brain stem depends on a dispersed firing in the efferent fibers from the cortex, except when a very high degree of synchronzation produces an outright convulsion. It can be supposed, then, that the degree of synchrony in sleep (and $\mathrm{m}$ pett mal) is too great for the temporally integrated control of muscle and too low to produce convulsions.

One consequence of such ideas is the conclusion that sleep produces relaxation as much as relaxation produces sleep. This makes possible a vicious circle, or cumulative reciprocal influence, that partly explains the gradualness of change from waking to sleep and vice versa. The drift from a dispersed finng to synchrony might be initiated within the cerebrum, if there is a sleep center in the anterior hypothalamus, cells in thus region would start to recruit others and might assume control of local cortical areas, which in turn would mean a decrease in the number of cells avalable for assembly action and a decrease in muscle tonus The consequent lowering of afferent excitation would further the same drift into hypersynchrony

In wakung, on the other hand, sensory stimulation would be considered to wean transmission units away from the pools of synchronous firing, each accession to this asynchronous assembly

- This one experimental fact alone might justify the treatment of sleep that is made $\mathrm{m}$ these pages The cortical transmussion that would be involved in a conditioned reflex, according to the phase-sequence hypothesis, depends on the series of assembly actions that is replaced in sleep by a local synchrony of finng, so even when a stimulus is applied directly to the cortex it could not elicit an adaptive response (in deep sleep) 
achon would increase muscle action and increase afferent exc1tation, and so on.

The experumental evidence has shown that a particular region has a crucial role in such changes, there is a wakang center, and Nauta has given reason for thinking that there is a sloep center as well. But such centers may be necessary only in coordinating the action of the relatively large mammalian cerebrum, and the same kind of process might occur in a smaller brain without special pacemaking centers. Also, even in man, wakung or sleep may not be necessarily initiated by changes in such centers Sleep is produced by cortical lesions (Davison and Demuth, 1945) and may be interrupted by nughtmares-a "conscious" activity. It may be profitable to consider sleep and wakang as an alternation of excitabulity to which any central nervous tissue is prone, but which can be coordinated throughout the whole cerebrum only by the regulating action of special structures in the hypothalamus. When the action of these structures is impared, disorganization of neural firung is possıble: producing emotional disturbance Interference with the sleep mechanism is accompaned by irritability (Nauta, 1946), and the irritability commonly seen when sleep is abruptly cut short, but which nevertheless disappears soon, as the subject becomes fully awake, indicates that between the intrinsic organization of sleep and the conscious organzation of waking there may again be an interval of disorganization of cortical fing.

The role of psychological infuence in determining the rate of drift from the diffuse organization to the intrunsic organszation appears to mean that some phase sequences are more firmly establushed than others. that is, are more capable of perpetuating themselves. The level of strmulation from sense organs affects the persistence, and a phase sequence may perpetuate itself by raising this level, as when one knits his brow and punches humself to stay awake.

Other phase sequences doom themselves to a short career by lowerng the level of stumulation, except for stimuly that make for hypersynchrony. When such a phase sequence is in command, we say that the subject "tries to sleep," seeking relaxation, darkness, and quiet But as we have seen there is a further cerebral factor, which becomes our next concern. 


\section{The Alternation of Sleep and Waking}

This is the factor of interest or motivation, which is provisionally translated into the stabulity and persistence of the phase sequence It appears first in the fact that no amount of muscular relaxation and quiet is likely to put the subject to sleep in the morning just after he has slept through the night; or, in other crrcumstances, in the fact that a good light, a loud voice, and a hard pew are not enough to keep one's eyes open Agarn, if the reader cares to make a little experiment and turns from this book to a detective story, costume thriller, or sentumental family plece-whatever his preference may be-he will find humself wide awake again though he sits in the same chair, with the same light and the same degree of quet, with the same degree of eyestrain in reading the same 26 letters of the alphabet (but happily contrived in other words). The problem raised here concerns the relationship between sensory input and the phase sequence, what characteristic it is, in this relationship, that tends to prolong the phase sequence and avert sleep

Another facet of the relationshup can be added at once: no matter how exciting a first reading of the detective story may have been, it will have lost this property on rereading unless considerable time has elapsed. To common sense, the reason is obvious The fun is gone when you know exactly what bappens next But it is not obvious theoretically-if the book kept one awake yesterday, why not today? If a particular sequence of sensory events tones up the wakung center, keeps hypersynchrony to a minumum, and mantauns pyramidal-tract control of the muscles, why should it not have the same physiological effects a second time?

In this, as I shall try to show, there is a clue to the sources of motivation, and "pleasure" In view of the defintion of motivation proposed in the preceding chapter, that it consists of the drectedness and persistence of the phase sequence, we must consider sleep to be the extreme case of a loss of motivation. From the discussion in the present chapter, it has appeared that sleep 25 not an isolated topic-not an isolated phenomenon dependent only on certain nucles in the hypothalamus and amounting only to an intermittent cessation of cortical function The frequently slow transition from unresponsiveness to full responsiveness (that is, from sleep to being wide awake) presents the 
same difficulty for theory that the slow development of sexual responsiveness does, and in his conception of "wakefulness of choice" Kleitman has evidently related waking and sleep to a much wider problem of motivation Let us see what approach can be found in the commonplace, but theoretically peculiar, fact that an event which is exciting and interesting on its first occurrence is likely to be dull and sleep-provoking on its second.

\section{EFFECTS OF MONOTONY ON LEARNED BEHAVIOR}

Certain changes in the form of Iearned behavior, resulting from repeated or prolonged stumulation, do not seem to agree with the fact that repetition is usually what established that particular form of response in the first place, and is also necessary to maintan it. Dunlap (1932) has shown how the deliberate repetition of a response may elimmate, mstead of strengthening, it Hovland (1936) bas described an "inhibition of reinforcement" from too many successive practice trals of a condtoned response As Hilgard and Marquis have remarked, Pavlov (1928) found it advisable to space trals wndely, m settong up a dufficult discrimunation; and it is well known that in ordinary learning massed practice does not have the same effect as distributed practice. Since a few seconds between trials should be enough to avert fatigue in the neural cell, one might think that longer intervals would only promote forgetting, but this does not seem to be true

Presumably related to these effects of repetition is the "fading" discussed by Gardner and Nissen - In prolonged traning, there are often periods when practice seems to bave a negative effect. The more he tries the worse the subject does. In man this is apt to go with a motivational disturbance, or loss of interest. One thinks little of it when repeated faulure reduces motivation, but after all, why should it? Also, even if the reason for this were self-evident, a deterioration in performance can take place when the human subject is still "trying as hard as he can," or in an anmal, workang for a food reward, that continues to be

- L Pearl Gardner and Henry W Nissen, "Simple discrimination behavnor of young chumpanzees- compansons wath human aments and domestic anumals" (In press, J Genet Psuchol) 
genunnely in need of food and apparently makes every effort to get it In almost any discrimmation training there are periods of deterioration of performance which are usually disregarded as due to chance but which, as Gardner and Nissen pount out, seem to require some further explanation

All these things might suggest that the connections involved in learning are (1) somehow weakened by beng activated, and need a period of recovery before they can function well a second time, but (2) are strengthened, unstead, when the pernod of recovery has been permitted Hull (1943) has shown the explanatory value of such an idea, though he has put the weakenng mfluence as a separate inhibitory process ("reactive inhibition") How are we to deal with the apparent weakening in the present theory?

Two possibulitres may be considered-that the cell-assembly tends to be disrupted by its activation, or that the relatronship between assemblies changes and affects the phase sequence. There are indications that both things may occur, and can be distingusshed one from the other by behavioral sigas; and, also, that the effect we are interested in is primarlly an effect on the phase sequence, not the assembly The argument is as follows:

The assumptions made in Chapter 4 about the formation of assemblies implied, first, that self-maintained activity in the assembly would stop after a very short penod (up to half a second). If nonetheless the arousing sensory stmulation persists without intermission, forcing a continued activity, the tendency would be to induce a change of frequency properties in the assembly. Some fractionation and recruitment would thus be expected ( $p$ 76). The facilitation delivered to other cerebral systems would then be changed, which means some change in perception. Theoretically, this appears to be exactly what happens in the notorious effect of prolonged staring at a word or repeating it over and over to oneself the famular perception tends to become something strange and unrecognuzable. Such effects can be regarded, then, as evidence of deterioration in the assembly due to continuous activation

But the assumptions of Chapter 4 also mplied, secondly, that when an assembly has become mactive it might be aroused again m a matter of a second or so, and after the same short 
interval aroused once more and so on, indefinitely. The behavioral evidence seems to support this, indicating that the single assembly or small group of assembles can be repeatedly aroused when some other actuvity intervenes. In vision, for example, the perception of vertical line must occur thousands of times an hour, in conversation, the word "the" must be percerved and uttered with very high frequency, and so on

According to the theory, then, the deteriorative effects of repeatung the same long senes of words over agam (loss of $\mathrm{m}$ terest on rereading a novel), or of repeating the same group of attempted solutions (loss of motuvation in problem-solving), should be in the phase sequence instead of the assemblies that compose it that $1 \mathrm{~s}$, in the relation between constituent parts, not in the parts themselves The same conclusion is indicated by the fact that the individual words of the reread story, or the detals of the unsolved problem, do not appear different (as the stared-at word does) but have an annoying sameness Also, a very ingenious experiment brefly reported by Lewm (1938) strengthens the argument:

The subject was required to write a word over and over untl the task became intolerable. The experimenter accepted his refusal to write the word agam, agreed to stop the experment, but casually requested the subject to write the word on the back of one of the work sheets, to identfy it. The subject did so without difficulty. Thus a change of set, or intention, banished fatigue. The assemblies directly necessary to writung the word may have been somewhat impared, but not to the point at which they could not function and the mental fatigue must have mainly concerned the accompanying assembly activities which constitute the subject's attitude or motivation The recovery in this experiment seems closely related to the increased output of industrial workers when a change is made in their attitude toward a monotonous task (Roethlisberger and Dickson, 1939). The theoretical significance thus is the same as that of the revitalizing effect of "insight" (a new combination of assemblies-p 134) on interest and motivation This effect is greatest on the first occurrence of the new assembly combination, on repetition, the stimulating value declines, just as when a joke or a story is heard for the second tume. 
interval aroused once more and so on, indefinitely. The behavioral evidence seems to support this, indicating that the single assembly or small group of assembles can be repeatedly aroused when some other actuvity intervenes. In vision, for example, the perception of vertical line must occur thousands of times an hour, in conversation, the word "the" must be percerved and uttered with very high frequency, and so on

According to the theory, then, the deteriorative effects of repeatung the same long senes of words over agam (loss of $\mathrm{m}$ terest on rereading a novel), or of repeating the same group of attempted solutions (loss of motuvation in problem-solving), should be in the phase sequence instead of the assemblies that compose it that $1 \mathrm{~s}$, in the relation between constituent parts, not in the parts themselves The same conclusion is indicated by the fact that the individual words of the reread story, or the detals of the unsolved problem, do not appear different (as the stared-at word does) but have an annoying sameness Also, a very ingenious experiment brefly reported by Lewm (1938) strengthens the argument:

The subject was required to write a word over and over untl the task became intolerable. The experimenter accepted his refusal to write the word agam, agreed to stop the experment, but casually requested the subject to write the word on the back of one of the work sheets, to identfy it. The subject did so without difficulty. Thus a change of set, or intention, banished fatigue. The assemblies directly necessary to writung the word may have been somewhat impared, but not to the point at which they could not function and the mental fatigue must have mainly concerned the accompanying assembly activities which constitute the subject's attitude or motivation The recovery in this experiment seems closely related to the increased output of industrial workers when a change is made in their attitude toward a monotonous task (Roethlisberger and Dickson, 1939). The theoretical significance thus is the same as that of the revitalizing effect of "insight" (a new combination of assemblies-p 134) on interest and motivation This effect is greatest on the first occurrence of the new assembly combination, on repetition, the stimulating value declines, just as when a joke or a story is heard for the second tume. 


\section{An Hypothesis of Excitability}

These considerations imply that the phase sequence contunually needs new content to maintain its organization and persistence * It appears that the individual assembly may be rearoused mdefinitely at short intervals, but that the phase sequence as a whole cannot be-it must continue to develop if motivation is to be maintained. "Develop" here means to lead to new combinations of assembles, which in turn means new perceptions, new insights, new ideas As we know, such events are exciting But why should new combinations be continually necessary to maintain the phase sequence, and with it a normal level of excitability? An answer can now be proposed which, in its wider signuficance, will also concern the nature of pleasure.

\section{AN HYPOTHESIS OF THE RELATION OF EXCITABILITY TO MEMORY}

To the psychologically nave there is no nddle about the fact that hearmg a story for the second time makes poor entertanment-the better you remember the detals of the first telling, the less your interest in the second. Common sense thus ascribes the change of excitatory value to a change in memory, and I shall try next to show how this idea may be utlized theoretcally.

Our question is why it is not possible to repeat a prolonged

- Thus conclusion is not contradicted by the fact that long periods of monotonous work are possible in the factory, where no change of insight or the like is possible. Habituation to monotony is apt to be accompanied by complex daydreaming, which means the development of a parallel phase-sequence activity, so the total pattern of cerebral activity is not at all repettive. The work of Roethlssberger and Dickson, referred to above, also shows that the behavior is related to the ancillary cerebral activity

Another possible objection can also be considered here According to the hypothesss presented in the following section, the infant's capacity for an endless repettion of some action or set of words is possible because the phase sequence is not yet well enough consoludated to lead to short-circuiting The following section modrfies the conclusions of this section in one respect, suggesting that it is only the highly organized phase sequence that cannot be repeated in detal, if the sequence loses in degree of organizaton with the passage of time, it can occur again Accordingly, the infant's repetitive action is considered to occur during the penod when organization is being establushed With completion of that process, the repettion disappears 
phase sequence in its enturety-why it must always lead to new assembly combinations, or else give way to some other cerebral activity. The answer may be that the mere occurrence of a particular phase sequence, once, induces changes at the synapse (memory) that make it impossible for exactly the same sequence to occur again, unless the synaptic changes have disappeared whth time. This can be made more specific, and made to comprise an explanation for the slow development of excitability that has been discussed in this chapter This is done by slightly elaborating the assumptons about the memory trace that were made in Chapter 4 . The implication is that an immedrate rearousal of a phase sequence is quite possible, but that the synaptic changes of memory then tend to make the sequence shortcircuit * and run off so quickly that it cannot hold the field long, If it is to endure, and remain coordinated with environmental stumulation, new elements not so well integrated with it must enter to delay its course.

Nowhere in the preceding chapters has there been any assumption as to the permanence of the synaptic connections establushed by learning (though it has been concluded that early learning has, in one way or another, permanent effects on later learning). This is because until now, at least, there has been no decisive reason to assume either that synaptic knobs once formed are permanent or that they deteriorate with disuse. If (1) they are permanent, one bas accounted durectly for the permanent effects of early learning, and may assume that forgetting occurs as an addition of new learning the establishment of further connections changing the tume properties of assembly function and so changing the faclitation delivered to other cere-

- The reader will recall that the phase sequence is "recurrent" and "antacipatory," contaning cyclical conceptual activities schematzed as

$$
A-B-A-C-B-D-E-F-D-E-C-F-I I \text {, etc }
$$

"Short-curcutang" might cut such a sequence down to

$$
A-B-D-H \text {, etc }
$$

That 28 , on repetition the sequence might touch only the high spots, after some synaptic knobs have detenorated, however, $D$ might be no longer able to arnuse $H$ directly $\multimap$ nly when $E, F$, and $G$ are also aroused 


\section{An Hypothesis of Excltability}

bral systems. The connections originally established would still be there, but the original habit would disappear, replaced by a new one Alternatively, (2) one might assume that a hnob disappears with disuse, which accounts durectly for forgetting, and, since we have seen reason (in Chapter 6) to think that new learning incorporates earlier learning as a "transfer" effect, the persistence of early memory can be accounted for because most early connections are maintained by the exercise they receive as they take part in the learning of maturity Either of these two assumptions might thus be made without disturbing the man structure of the theory

But for the problem now being considered, a solution may be found if we can adopt assumption 2, that an unused connection decays, or, better yet, if we can assume that a very frequently and long-used connection becomes permanent, but that there is a stage in the development of synaptic knobs before which the development is reversible The old, long-established memory would then last, not reversible except with pathological processes in the bram, less strongly established memories would gradually disappear unless remforced.

Now: suppose a man picks up the book he read last week The ttle and the first paragraphs recall the story-the phase sequence runs off quickly, short-circuited, leaving many of the cell-assemblhes briefly refractory before they can be aroused by the rest of the printed matter No cooperation is then possible between sensation and central facilitation, the latter is too far ahead Something of this sort would account for the contunued need of some degree of novelty to maintain wakefulness of chorce. The thoroughly famular arouses a well-organized phase sequence, the very fact that it is well organized means that it runs its course promptly, leaving the field for less well-established sequences. and so, from this theoretical point of view, one would find behavior dominated always by the thought process that is not fully organzed-one that is achieving a new organization or one in which synaptic decay makes it necessary that organizaton be reachieved.

Thus means that the subject will not linger over the thoroughly familar event even though this is the one that is most capable 
of arousing and controlling central processes, the control may be momentary only The totally unfamiliar does not arouse a phase sequence, so "interest" and "motivation" are likely to be preoccupied by whatever is new in the combination of familiar events, and by events that produce some frustration or some fear (which tend to break up the phase sequence-Chapter 10) Rereading a half-forgotten novel would thus be possible, immedrately after the conclusion of any exciting or interesting series of events a repetition would not be exciting (or not as excitung, depending on the degree to which synaptic changes have produced short-circuitag $\rangle$, in the following minutes, hours, or days the subject's responsiveness to that sequence of events would steadsly rise as the synaptic changes regressed Sumlarly, the psychological function of a night's sleep may be to permit a certain decay in the particular connections that were established or strengthened by the prevous day's behavior, so that the behavor may recur again as a cooperation between sensory and central processes.

If it should be true that such reversible growth processes occur, the problem of the slow changes of excitability might thus be solved. Is the idea at all plausible? One is accustomed to thinking of the nerve cell as a static structure (as it is after being fixed and stained, and of growth as a very slow process. Yet it may be recalled that Cajal (among others) comectured that the change at the synapse in learnung is an amebord outgrowth of the cell (see, e.g, Freeman, 1934, footnote p. 28), which might need very luttle time for its occurrence

Cajal's proposal has been disregarded by psychologists and no longer figures in discussions of leaming Presumably, this is partly because the whole theory of synaptic connections and resistances fell into disrepute some tme ago. But it has been seen (Chapter 1) that modern neurophysiology makes it possible to reopen this entrre question, and so Cajal's adea cannot be at once dismussed as fantastic.

As to the tme needed for an outgrowth of the cell wall, we may note (1) that the actual distance involved may be veny short indeed, and (2) that reverberatory activity in the assembly would serve to prolong the tme in which a structural change 


\section{An Hypothesis of Excitability}

could be completed An amebond outgrowth would be reversible, but the mere absence of the electrochemical miluence which produces the outgrowth might not act as forcefully and promptly as its presence So it is possible to assume, in the light of the psychological evidence, that synaptic decay occurs slowly and perhaps is never quite complete. Thus each repeticon of the amebord change at a particular point might leave a hugher residual Jevel, and with a frequent occurrence the outgrowth might become permanent These assumptrons then would correlate durectly w2th the fact that memory can be qujaldy established but declnes slowly, and with the fact that there is apt to be hattle or no forgetting of the long-practiced response.

It is proposed therefore that some such postulate should at the least be considered sernously, for it would help us to deal not only with (1) the facts of learning, but also with the formidable defficulty of understanding (2) the reversals of motivation discussed in this chapter, (3) the length of time over whuch excitabulity (rather than excitation) may on occasion increase, and (4) mental fatigue without a fatigue of the neurans and effectors that enter directly into the response. The assumptions discussed above do not belp particularly to deal with a bebavioral excitation (except to suggest that its tume course may depend on the rate of establishment of new-and reversible-connections), but they do appear to account directly for Jong-term changes in excitabsity, and in general for the psychological component in sleep and waking in sexual responsiveness and so on, and in the effects of novelty on these condrtions. It is of course evident that some degree of novelty, combined with what is predominantly familiar, is stimulating and exciting over a wide range of activities-from sexual responsiveness (Beach, 1947a; Kinsey, Pomeroy, and Martun, 1948) to the appreciation of paintang or music, or the pleasures of exploretion

I do not propose to discuss the neurological assumption further, but only point out once more that the decay of memory allows what has been experienced to become relatively novel again and, therefore, exciting, so that the necessity of a lapse of time between repetitions of some activities can now be fitted into a more general statement of the conditions of pleasure. 


\section{THE NATURE OF PLEASURE}

The theory that has been developed implies that pleasure is not the activity of partcular structures in the nervous system, and not even a particular knd or pattern of cerebral arganization, but fundamentally a drected growth or development in cerebral organization. It is thus necessarily a transient stato of affars in which a confluct is being reduced, an mopient disorganization being dissipated, or a new synthesis in assembly action being achieved The preceding section has tried to explain why such states of affars should dommate cerebral processes, in other words, to explain why pleasure should have the peculiar place in behavior that it has, without covert recourse to an an1mistic consciousness, just asethe discussion of emotional disturbance and pain, in other chapters, has tried to provide mechanlcally or determmistically for the avoidance of certain activities and situations

Those sensory conditions are called pleasant, then, which contribute to the current development in the cerebrum, or which consist of the decline of a sensory process that interferes with development. If going to sleep $1 \mathrm{~s}$, as has been suggested, the establishment of an "intrunsic" organzation, the process according to the definition above should be pleasant-as in fact it is, and to the drowsy subject those stumuli are pleasant which actively support the development of hypersynchrony But some eight hours later, as the subject is waking up, the pleasant sights and sounds are those that "catch his interest" and promote another land of organization, without hypersynchrony. The same stimulus is thus pleasant at one time, not at another, when the event that could be interesting later occurs too soon in the waking-up process, and disrupts hypersynchrony without promptly establishing a phase sequence, it is the very reverse of pleasant

In vew of the discussion in the preceding section of the effect of short-circuiting on motivation, there is a paradoxical implication here Sensory events should not support the phase sequence too strongly, so that it runs its course immediately and cannot continue to dominate association-area activity. The well- 
developed phase sequence depends, for a contnued exstence, on repeated-checks from the environment Hence, as we saw in the preceding section, the preoccupation with what is new but not too new, with the mildly frustrating or the mildly fearprovoking Many adult sports of course depend for ther pleasure on the presence of some danger, and both Woodworth (1921) and Valentme (1930) have remarked on the tendency of children to seek out the frightening situation in which the degree of emotional stimulation remains under control and can be terminated at will This seems wholly parallel to an adult's reading of "thrillers" and ghost stones.

Simlarly, problem-solving involves frustration, and many of the actinsties that are sources of pleasure actually depend on problem-solving-not only in games like bridge or chess, but also, as Woodworth and others have remarked. in art appreciation and novel reading

In music, again, the dissonances that are harsh and disruptive at first become pleasant as they become more famliar, but finally are dull and borng (Chandlex, 1934) The course of events is first, a dissonance with too much confluct to elicit an organized cerebral action, secondly, with the establishment of new assemblies, or modification of existing ones (due to repeated stımulation by the new tone combination), an organized activity is aroused, in which, however, some conflict remains, and, finally, organization reacbes a point at which the sensory stimulation no longer offers any check to the phase sequence, and pleasure has disappeared. This phenomenon in music might be subsumed quite well under Woodworth's generalyzation concerning the role of problem-solving in art appreciation, but still more easily by the present formulation What I have sought here is a generalization that would subsume not only the problem-solving factor, but also the effects of novelty and of a mild degree of fear, in contributing to pleasure.

In its relation to the rest of the theory presented in this book, the discussion of pleasure has had two significances First, it is umportant to show that the theory of emotional disturbance in the following chapter does not require that all confhet is unpleasant and grossly disruptive of behavior, on the contrary, 
some degree of confluct is stimulating and necessary to the maintenance of normal responsiveness to the environment

Secondly, whether it is eventually found to be adequate or not, this discussion of pleasure together with the bypotheses of the nature of pain and emotional disturbance represents my attempt to be rid once and for all of the little man inside the skull who approves of some sensory events relayed to hum by the nervous system, disapproves of others, and guides behavior accordiogly who encourages stmulation of the afferents from the genitalia in his crafty and teleological aim to perpetuate the species, and who for the same purposes becomes violent when $C$ fibers are stumulated or when he foresees that they are goung to be By some such approach as the one suggested, it may become possible to understand the directedness and order in behavior, and the variablity of motuvation, as produced by neural functioning alone. 


\section{O. Emotional Disturbances}

The discussion of emotion has been about as confused as that of any topic in psychology, partly because the terminology is often equrvocal and pertly because tradition carries great werght in this part of the field and it is hard to keep a modern point of view consistently The present chapter deals with emotional dusturbance instead of emotion, for reasons to be made plain, and the discussion is limited in other respects It is not concemed mainly with sham rage and the hypothalamus, or with emotion as a kund of awareness, or with emotion as it may exist in states of quiet affection and the like "Emotional disturbance" here is used to refer to the violent and unpleasant emotons, roughly, and to the transient imtabulities and anxieties of ordrnary persons as well as to neurotic or psychotic disorder. Let me offer what justification I can for such an arrangement of topics.

\section{EMOTION AS DISTINCT FROM SHAM RAGE}

Since the work of Bard (1934), the phenomenon of sham rage and its dependence on the posterior hypothalamus have held the center of the stage in the theory of emotion But the analyses of Lashley (1938c) and Masserman (1942), particularly where they have dealt with the clinical data that were supposed to establish the diencephalon as the seat of human emotion, have limited the emotional functions of this region to a motor untegration An equally significant modification of his earher work has been made by Bard (1942) humself. He has reported that rage can be evoked from cats in which the pos- 
tenor hypothalamus only has been destroyed-a most important observation.

Thus, "rage" is not localized in the hypothalamus. Also, the rage of the decorticate cat is markedly different from the normal, so the name "sham rage" was properly chosen and should be taken literally. Sham rage is different in its time properties, lacking duration or after-discharge following stumulation, it is different in the rigidity of its pattern, having almost none of the normal varrability of motor expression, and it differs radically in the limited range of stimulation that is adequate to elicit it Also, Spiegel, Miller, and Oppenheimer (1940) have reported the production of rage by lesions of (1) tuberculum olfactorium, (2) hippocampus and fornux, and (3) anygdalond complex, and slight, transient signs of rage from damage to (4) the pyriform cortex.

Apart from these considerations, there are other emotional processes besides those that produce overt rage behavior It is true that fear as well as rage has been evoked from the decorticate cat, but apparently only by auditory stumulation and with considerable difficulty. Further, strong emotional disturbance can exast in the intact anumal without producing either clearcut attack or cowering and flight. The problem of emotion, in short, is not simply the problem of the motor pattern of sham rage (or "sham fear") It is of course essentral to have discovered the unexpected extent to which rage and fear patterns may appear in the decorticate anmal Bard's work was one of the most umportant single contributions to understanding emotion But, given that knowledge, we must go on next to ask how the hypothalamic expression is modified by the presence of a large thalamus and cortex, how learning affects emotional behavior, and the relationship of emotion to perception and thought.

\section{EMOTION NOT A STATE OF CONSCIOUSNESS}

Traditionally, emotion is an awareness, an event in consciousness. Here, perbaps more than anywhere else in psychology, a traditional interactionism (which is animism) tends to persist. The afferent excitation is thought to produce a feelng or awareness, and that feeling then acts on the nervous system-it must 
do so, according to such ideas, for it is the feeling that makes the subject sweat or tremble or run away, and the sweat glands and the legs are controlled by nerve fibers.

Just such an inconsistency of thought has led to an endless, and pomtless, debate on the James-Lange theory of emotion What James and Lange were accounting for, again, was that emotional feeling or awareness, they postulated that the awareness is a set of sensations, that the awareness follows and does not cause emotional behavior ("I see the bear, I run, I feel afraid"). Therr critics proceeded to show that emotional expression is still there in the dog whose sensory processes have been interfered with, and thought this a refutation of the theory. But such an argument is totally urrelevant, James did not say that emotional behavior depends on sensations from the limbs and viscera.

It is equally irrelevant to show that emotional expression is centrally organized, in the hypothalamus If James had rassed the question at all, he must have assumed something of the sort; remember that what be denred was that consciousness intervenes between stmulus and response Therefore, showng that the "higher" centers of the cortex are not needed for the response is clearly support for his position. The extraordmary non sequstur of such criticisms, made by some very distingusshed critics indeed, would not be possible if there were not the immutable idea thet only emotional awareness or feeling can produce emotional response If the response is there, the feelng must be also. Such illogic, assuming James to be wrong first, in order to prove him wrong, is the clearest evidence of the hold traditional ideas have on psychological thought.

But we must get nd of the tradition both for scientufic consistency and because psychological observers have been unanimous in denying that there is any special, fundamental category of consciousness that can be called emotion. "Emotsonal experience is a hughly varable state [and] often partakes of the complicated nature of a judgment" (Landis and Hunt, 1932) I have reviewed the evidence on this point elsewhere (Hebb, 1946a) and need not go into it again. Since it seems that the term ernotion does not refer to a special kand of event in consciousness, and since in any case we must not slip into the in- 
consistency of treating an immaterial awareness as a causal agency, the term is not very useful in its traditional significance. At the same time, we must postulate that the disturbances of emotional behavior have a neural ongm, and the term emotion still can be useful to refer to the neural processes that produce emotional behavior

It is important to be clear that in this discussion "emotion" is a reference to the hypothetical neural processes that produce emotional behavior, explicitly, it refers neither to an immatenal state of consciousness nor to the observable pattern of emotional behavior.

\section{EMOTION AS A DISORGANIZING INFLUENCE}

The third point to be clarified is the distnction between emotional disturbance and those processes (also called emotional) which are mherently organizing and motivating. A paper by Leeper (1948) has urged that all emotion be considered to have such an integrating function He has expressed a commonly held point of view, and has at the same tme I believe clouded the issue with which he dealt His position demands consideration.

Leeper has shown effectively that regarding emotion as disorganzzing has led some of us into an mconsistency, but he has proposed another inconsistency to take its place He points out that others have first defined emotion as a disorganization of behavior and have then gone on to give mother-love as one example of emotion This is certainly inconsistent But how is it different logically from defining emotion as something that makes behavior more efficient and at the same time giving, as examples, the stage fright that ruins a performance, the anger that makes a boxer less skillful, and the mental depression that makes a man unable to earn a lnving? I must add at once that Leeper anticipated this criticism. He answered it by sayng that, though emotion may sometumes disorganize, the disorganization is not characteristic because it occurs only (1) when emotion is extreme, or (2) when the emotion conflicts with some other motivation As far as one can see, however, the first of these 
explanations depends on a mistaken use of analogy, the second begs the question.

I. It might be doubted whether emotion is disorganizing only when extreme A touch of shyness or of self-consciousness, for example, may sometmes rum one's conversational skills Leave this to one side, however, and accept the assumpton that only strong emotion disrupts behavior

The idea that such an effect does not indicate the true natire of emotion depends on this analogy An excess of salt or oxygen has toxic effects, "but we do not use this as a means of determining the normal functions of such products. The same logic, then, should apply to emotional processes" (Leeper, 1948, p. 15) But we must really use the same logic We must, that is, ask the same question in both cases. The queston does not concern the "normal function" of emotion, but what emotion is No one doubts that fear has a useful function, anding survival, the question 1s, how? Might fear not be a simple disrupter of behavior and still be useful, if the disruption is manly of the behavor that gets us in trouble? Emotion could then be disorganization and still have its survival value, because the disorganization is selective, eliminating some actions and allowing others to take their place.

If we accept the analogy between emotion and salt we still can say that emotion is disruptive of behavior, that it generally has a useful function when it occurs in moderate degree and bad effects when there is too much of it-exactly as with salt or oxygen

2 In discussing the stage fright that keeps a pianist from giving a good performance, Leeper says the emotion is not a disorganizer of behavior because, by itself, it would produce only flight-a well-organized pattern of behavior This seems to beg the question, since the pianist neither runs away nor stays to play well Leeper says it is not the emotion that produces his incoordinations, but the conflict. But if there were no conflict at all, would there be any emotion? Are the confluct and the emotion quite independent?

The difficulty here is clearest by Leeper's own critenon of "organization." He points out first that any directed behavior means the suppression of conflicting tendencies, so conflict is not 
ipso facto disorganization. The test is "whether [an] interference is relatively chaotic and haphazard, or whether the suppressions and changes of subordmate activities are harmonious with some main function that is being served." The trembling, palmar sweating, disturbance of breathing, and incoordinations of the planist's fingers, obviously, are not harmonious with the main function being served-until he actually runs away Could we regard his emotion as solely organuning even then? See what this imples If we are really consistent in sayng that any disorganization in emotion is accidental, that emotion is essentially an organuzer only, then a well-organized avoldance is at least as much an instance of fear as an ill-organized one. Consequently, the pianist who anticipates the possibility of stage fright, and calmly decides to keep away from the stage, provides as good an example of emotion as the one that trembles before an audence We have deliberately got rid of any criterion of emotion except that it "arouses, sustains, and durects" behavior We have equated avoidance and fear, aggression and anger. But these surely are not identical

The upshot of such an argument is to broaden the category of emotion so much that it includes all psychological processes. We should then have to find another name for the distinctive event that-at present-is called emotional.

How are we to find some halfway point between this position and the one that Leeper has criticized so effectively? I think it is evident that Munn, Young, Dockeray, Woodworth, Landis, and so on (authors criticized for treatng emotion as disorganization), were after all not as absurd as Leeper thought them; at the same time, he has made untenable the proposition that "emotion" simply means a disruption of behavior.

A way out, I believe, can be found if we do two things: first, stop talking about emotions as a single, fundamental kund of unitary psychological process, and separate (I) those in which the tendency is to maintain or increase the original stumulating condations (pleasurable or integrative emotions) from (2) those in which the tendency is to abolish or decrease the stimulus (rage, fear, disgust), including, however, depressions in which the organism may discover no way of escape from the condition giving rise to the emotion. Secondly, if it is assumed that 
stmuli in class 2 above are essentially disintegrative, it must also be recognized that the disintegration in rage or fear is often incipient or potentral and likely to be successfully averted by the aggression or avoidance of the subject.

As to the first of these points, we have no need to bring together pleasure-seekng and melancholia, mother-love and temper tantrums, always in the same single category-"emoton." I have, in the preceding chapter, discussed pleasure as a special problem, and I propose in this one to try to deal with a quite different sort of process. Since "emotion" undoubtedly will often continue to mean affection or pnde as well as anxiety or shame, "emotional disturbance" is a better way of designating the subject matter of the present chapter.

\section{RAGE AND FEAR IN CHIMPANZEES}

The theory of emotion proposed by Watson (1924) is by now classical It beld that there are three innate emotions, rage, fear, and love rage aroused by a restriction of physical movement, fear by a loud noise or sudden loss of support (and presumably, pain), love by stmulation of the genitalra These strmuli may then serve to condition others. If a child fears animals, it is because he has been scratched by a cat or knocked over, perhaps, by a frnendly dog whle learning to walk As soon as one tries to apply this theory to the emothonal disturbances that are actually observed in children, serious difficulty shows up, it is not really plausible, and it has been subjected to powerful criticism by Dennis (1940) on rage, by Jones and Jones (1928) on fear, and by Valentune (1930), also on fear But it has been hard to document the criticism with experimental ovidence, for two reasons. First, it is not safe or socually permitted to arouse a strong degree of emotion in human subjects for expermental study Important as the topic is, consequently, wo have surpnsingly little exact information about the causes and consequences of human emotion-above all, of adult emotion. Secondly, when one studies emotion as it occurs socially, outside the laboratory, it is almost impossible to know the antecedent conditions as they must be known if one is to understand the present responses of the subject. 
But neither of these objections need apply to the study of emotion in animals, and the chimpanzee fortunately has an emotonal repertoire that is very like man's The thmgs that annoy us are apt to annoy him, and he shows his annoyance much as we do, his fears in some respects are astonishingly like man'sin what is feared, in complexity of the causes of fear, in the relation of fear to intellectual development, in resistance to extmcton or reconditioning, and in the marked individual differences between one subject and the next. Even more, there are data on what were with practical certanty two cases of neurosis or psychosis in mature chimpanzees whose histories were known from birth or very early infancy, one born in the Yerkes Laboratories of Primate Biology and one captured in the first year of life. These anumals of the Yerkes colony can be studied as human subjects cannot. Their histones are known in detal, being recorded in individual dianes With these data, one is in a position to see just what complexity of response must be provided for in a theory of emotion, chronic as well as acute.

The causes of rage may have nothing to do with physical restraint of movement. When the chimpanzee Dita, in heat, would sit where he could watch her from the next cage but one, Don seemed calm (If not content), but he had a temper tantrum repeatedly whenever she left the outer cage for an mner room where he could not see her. When Mona had a nowsy temper tantrum because Pan had stolen a peanut from her, Pan was finally enraged to the point of beating her up A chimpanzee may be angered by a reproof, by being startled, or by being obhged to look at something unpleasant such as a model of a snake These causes act "spontaneously", that is, the emotional disturbance does not have to be learned, any more than a human being must learn specially to be angry at disturbance of the peace, at being shown something nasty, or at being momentarily scared by a practical joker (Hebb, 1945a)-although, as we shall see, a certain kind of learning, in infancy, may have to have been established first.

The causes of fear include not only loud noise or sudden fall, but snakes with which an animal has never had contact (this can be known certainly with some chimpanzees reared in the nursery), solitude, and the strange or mysterious. McCulloch 
and Haslerud (1939) have analyzed such fears in a young chunpanzee reared quite apart from others, and were thus able to show that they are not necessarily acquired by "social condition$\mathrm{ng}^{\prime \prime}$ or imitation I discovered accidentally that some of the chimpanzees of the Yerkes colony might have a paroxysm of terror at being shown a model of a human or chımpanzee head detached from the body, young infants showed no fear, increasing excitement was evdent in the older (half-grown) anımals, and those adults that were not frankly terrified were still considerably excited. These individual differences among adults, and the difference of response at different ages, are quite like the human differences in attitude toward snakes, the frequency and strength of fear mcreasing up to the age of seventeen or so in persons who had never been murred by a snake (Jones and Jones, 1928) The increase fits in with the conception that many fears depend on some degree of intellectual development, and so with the idea that the range of such fears is characteristic of "higher" anumals like man and chimpanzee (McBrde and Hebb, 1948)

The observations using detached heads were then followed up by others, using various stimuli. an isolated eye and eyebrow, a cast of a chmpanzee's face, a skull, a cured chmpanzee hide, an anesthetzzed chimpanzee.

Two things appeared first, that such stimulation may be primarily a source of profound excttation, rather than specifically fear-producing, the excitation was usually followed by avoidance, but sometımes by aggression, and sometimes it produced a marked autonomic reaction combined with apparent friendliness On occasion, a confusing muxture of all these reactions was seen

Secondly, this primitive excitation appeared to be fully parallel to the human emotional disturbance that may occur at the sight of a badly deformed face, in watching a major operation, dissection, or autopsy for the first tme, or as a result of contact with a dead body (Hebb, 1946b). This human disturbance, like the chimpanzee's, is not specific-it may take the form of nausea, of vascular disturbance and dizziness, of disgust or fear, or may even faclitate anger ( $e g$, at "desecration" of the dead) It is important to recognize individual dufferences of sensituvity 
to such experiences, and the loss of sensituvity with repeated exposure (which is presumably an effect of learning), but this does not mean that the responses are wholly leamed The evidence is very definitely to the contrary: the degree of excitation is lukely to be strongest on the first exposure, provided certann other experiences have preceded On the other hand, the disappearance of response with repetition must be an effect of learning, and I believe we must assume that it is learning also that establushes any integrated response to such stmul, transformug a nonspecific emotional disturbance into an organized flight (distroance plus a tendency to firght then constituting fear) or aggression (constituting rage).

The complex ongin of emotional disturbance may be clearest in the infant fear of strangers About the age of four montbs the chimpanzee reared in the nursery, with daly care from three or four perspns only and seeing few others, begins to show an emotional disturbance at the approach of a stranger (Hebb and Riesen, 1943) The disturbance increases in degree in the followng months This is "shyness" but may become much more violent than that term would usually imply. It commonly shows up in the human baby about the suxth or seventh month (The age difference is in keeping with the fact that chimpanzee development generally takes only about two-thirds of the time for the corresponding human development ) Everyday expenence tells us that shyness does not occur in the human chuld that is continually in contact with strangers, and that it is very strong, as a distrust or fear of "foreigners" (even in adults) in isolated communities. We might then suppose that the excitation out of which the fear develops is aroused innately at the sight of a stranger, but extungurshed if enough new faces are seen with no accompanying injury. But this supposition is wrong there is definite evidence that the response is not unately established

Dennis (1984) pointed out that human patients, congenitally blind and operated on to restore vision, have shown no emotional disturbance at the first sight of a human face Chimpanzees reared in darkness, and brought into the light at an age when the response normally would be at its strongest, show not the slightest disturbance at the sight of either frnend or stranger. But some tume later, after a certain amount of visual learning 
has gone on, the disturbance begins to appear exactly as in other anumals.

So it appears that the emotional dusturbance is netther learned nor mnate a certam learning must have preceded, but given that learning the disturbance is complete on the first appearance of certain stumulus combinations Dennis (1940) has also made it clear that temper tantrums are not learned, and still require that other things have been learned first. We are accustomed to think of any particular response as ether learned or innate, which is apt to be a source of confusion in thinking about such things as far apart as an insightful act or neurotic behavior-1s the response mherited, or acqured? The answer is, Neither. etther Yes or No would be very misleading. The irrational emotional disturbances of man and chimpanzee are fully dependent on learning, but are not learned in the usual sense of the term.

On the other hand, in order to account for the specific processes of rage and fear, and the integrated, adaptive responses of aggression and flight, we must $I$ believe give learning its role. This point will be returned to after broadening the field of our unuury by considering two cases of mental lliness in chunpanzees.

\section{MENTAL ILLNESS IN CHIMPANZEES'}

Now, before turning to the mechanism of these responses, let me report two cases of apparent neurosis or psychosis in the chimpanzee. Here again we have the advantage of knowing more of the prevous expenence of the patients than is possible with most human patients in the clinic. We have no verbal recollections to go by (recollection in clinical cases, after all, is apt to be unreluable), but the anumals' experience, in cage life, is much less varied than a man's would be and-once more-the chmpanzees of the Yerkes colony bave been under daly observation by trained observers who have faithfully recorded at the time of its occurrence any observed llness, unusual expenence, or peculianty of behavior.

We have further, in the particular cases to be discussed, the invaluable asset of control subjects It happens that four chumpanzees were reared together for a large part of the develop- 
mental period, in the same circumstances Alpha, Kamb1, Bula, and Bumba Alpha and Bula were born in captivity, Kambi and Bimba were captured wild, at about nine months of age. Nothing was recorded, and nothing could later be recalled, by the trained workers who reared them, that'would account for any great differences in the adult personalities of these four chimpanzees Yet, by the time of adolescence, one of the four (Kamb1) was a hypochondriac-as far as behavior could show, and one other (Alpha) in full maturity suddenly developed an extreme phobia Let me give these histones in more detail.

Alpha was born in 1930, the first chimpanzee to be born in the Yerkes colony She was taken from her mother immediately and reared apart from other chmpanzees untl she was one year old (Jacobsen, Jacobsen, and Yoshoka, 1939), She then was brought into the laboratory, as one of the group of four to which I have already referred The menarche was at age eight, and her sexual behavior with males has always been enturely normal The concensus of the staff was that Alpha, as she became mature, had a strong likng for human company, was not particularly bright and was very obstunate but still a good expermental subject Otherwse she was just a chumpanzee Chmpanzees, like men, have their individual peculiarities, and no great prognostic significance was attached to some small peculiarities of Alpha'salthough, in the light of her later psychuatnc problem, some of the peculiarities were peculiarly Freudian Alpha would definitely not have anything to do with any carrot with a forked root, nor usually with any long cylindrical vegetable such as okra, though when carrot or okra was cut up before her eyes she would accept and eat the pieces immediately. Finally, she had a marked tendency to masturbate, frequently against the water faucet projecting from the wall of her cage, whenever a human being came in sight.

In 1942, when Alpha was twelve (corresponding roughly to full maturity), a serious disturbance of behavior appeared. With no warning signs, she suddenly refused all solid foods, despite being very hungry. It then appeared that she would eat at once if food was cut small for her, and a number of tests showed conclusively that she was volently afraid of contact with any piece of food more than an meh or so in length or thickness Con- 
siderations given elsewhere (Hebb, 1947) lead me to believe that this behavior cannot be sufficiently explained as due to injury, as from a thorn or stinging insect in the food, though such event may well have been the precipitant. The fears fluctuated considerably, but waned gradually until they had completely disappeared two years after the onginal onset, then, after another year, they reappeared with equal suddenness and severity (this second episode clearng up more promptly, however).

A further phenomenon, of the greatest importance for showing the parallel with clmical cases, was observed durng the first of these two neurotic or psychotic episodes. Four months after the first onset, when the fear of food had greatly decreased, Alpha out of a blue sky developed a strong fear of me, later a violent aggression, and then (having been soaked with water from a hose for her persistent attacks) became quite fnendly, at the same tume reverting to her fear of food.

Two friendly critcs, on the occasion of the original report of this case, were able (with senous intent) to diagnose the case and explan the cause of llness Alpha, in short, was in love with me, the earher fear of forked carrot and of cylindrical objects, and the masturbation agaunst a projecting water faucet, were not the only Freudian features of the case. The aggression occurred because Alpha had had to repress her unmardenly desires, with an inevitable result This is an interesting idea, liking chimpanzees (or some chimpanzees), it would please me to think them capable of such delicacy, such capacity for repressing vulgar desire-m short, to think of chumpanzees as having Superegos

But if Alpha had a Superego its operation was rather hit-andmiss, and in many of her dealings with other chumpanzees (or with the staff), and in some of her personal habits, there was little sign of self-imposed restrictions If Alpha was in love, it must have been with someone else, and her emotional disturbance had no relation to fluctuating level of sex drive, which in chimpanzees is durectly correlated with the menstrual cycle It might be amusing to try to psychoanalyze Alpha, but it seems it would not be very profitable etther as therapy or as a contributon to knowledge

The second history is quite different 
Kambi was captured at the estimated age of nune months and reared constantly in the company of other females of her own age. From eughteen months of age onward, she was one of the group of four-AJpha, Kambi, Bula, Bumba Morphine addiction was brefly established at the age of eight (Spragg, 1940) She showed almost none of the usual sex play before menarche (at age nune) and copulated once only after it-although she showed the physical signs of coming into heat penodically, with the menstrual cycle. She was spayed at the age of twelve for exper1mental purposes, with no apparent change in over-all personality.

To summarize Kambi's behavioral disturbance first in clinically intelligible language, she gave the impression from early childhood of being introverted, with a very strong preference for human company, and subject to periods of depression. Durng adolescence, the depression when it occurred was extreme The attacks continued after maturity and often lasted for months at a tume. Her depression was sometimes initiated by a somatic Illness, when it did begin so, it did not clear up with the Illness but contunued long after."

The actual endence of depression is as follows Ordmanly Kamb1 was easily annoyed by fallure to get something she wanted, but she was always responsive to (and acted in a way that was likely to get) attention from the human staff, definitely more interested in such attention than in food, but ordinarily gettung enough to eat. But then for a period up to six or eight months in length she would sit day after day with her back hunched up against the wall of her cage, never seeking attention and rarely responding to human overtures, and almost totally uninterested in food so that if she were not caged alone she might have starved definitely a different animal.

Once more the case shows a Freudran element, in Kambis

-I am indebted, for some of the observations in this summary, to Dr Henry W Nussen, who knew Kambl thoroughly in her infancy and at maturty. It was he who obtaned her in Africa and brought her to the Yerkes colony with Bimba and a number of other young animals, and later had personal charge of their reanng I should emphasize that the descripton is a convenuent way of reporting behovior and does not necessanly imply anything about consclous states Kambi in short acted as a depressed introverted humen might act. 
lack of sexual motrvation (which has, however, been observed in other animals at the Yerkes laboratories, with no sign of psychotic involvement). But no traumatc sexual event can be adduced to account for this. The one copulation that was observed was quite normal, and Kambi's lack of sexual responsiveness had appeared much earler Disturbed ideas about sex are common in human mental llness, but so are disturbances of eating and sleep, and there is usually no evidence to show that sexual conflict occurs first and is the cause of the more profound disturbance of which it is part. In Kambi's case there was no evidence of sexual conflict at all-simply a complete lack of apparent interest.

The emphasis in these two hustories of chumpanzee psychoss, or neurosis, is on the absence of any particular experience to which the behavioral breakdown can be ascribed. But I do not suggest for a moment that experience is not a factor, $I$ shall urge later that expenence is a factor in almost any emotional breakdown. The point here is that if it was the main factor, in these two cases, it must have operated in a way that we do not at present understand at all, and quite unluke the mechanism of the "experimental neurosis."

The fact to be kept in mind is that we have two chimpanzees as control subjects (in an unplanned experiment, since no one foresaw the later course of development in Alpha and Kambr, but still an excellent experiment). After capture, Bimba had the same general experiences as Kambi (both captured at the estrmated age of nune or ten months), in every respect that our present knowledge would suggest is important There is no basis for postulating some special trauma for Kambi before she came under psychological observation, for the diaries of the two animals show that for the first three years of captivity it was Bimba, not Kambi, that was less well adapted to her environment more fearful, less 'trusting, less cooperative in an experiment-until the age of four, when the roles were gradually reversed

Again, Alpha and Bula were both born in captivity, and though Bula was left with her mother until the age of fourteen months whereas Alpha was removed at birth, there is little sign 
in the diaries of the subsequent four or five years to show that this had any effect on their later adjustments.

Experience, and expenence alone, may have been the decisive factor in the breakdown of two of these four animals at maturity, but, if so, it operated in some way that is quite mysterious in the light of our present knowledge of behavior. Freudian theory has the credit of recognizing the exustence of a kund of learning that causes, apparently, no immediate emotional disturbance and yet may contribute to one much later. Such learning undoubtedly occurs, and may well have been involved in Alpha's or Kambi's llhess, but if this is to be postulated we must find some way of incorparating it into other learning theory-not as an ad hoc assumption specially made to deal with mental illness.

\section{MECHANISMS OF EMOTIONAL RESPONSE}

What I believe to be the mechanisms of primary emotional disturbance have been discussed in precedmg chapters, together with a mechanism of emotional learring It remauns to pull the earler discussions together and to try to show how a mechanism of learaing might transform emotional disturbance, as a breakdown of adaptation, into the adaptive responses of attack, flight, and so on that 2s, how anger or fear might develop out of an undifferentrated emotional disturbance, and how a fallure to develop an adaptive mode of response might constitute neuross.

The aspects of emotional disturbance that mainly determine this treatment of the problem can be summarized as follows (1) the great variety of causes of disturbance, ranging from an unfamiliar combinatson of famlhar things (fear of the strange) or an zaterruption of sleep, to huager, nutritzond deficiency, or withdrawal of a drug from the addict, (2) the fact that a single cause may produce anger, fear, or nausea and famtness, in the same subject at dufferent tmes, or in different subjects; (3) the great variety of expression even of a single emotion; and (4) the dufferent ways in which the expression of emotion changes, as the subject is habituated to the stimulatung conditions

To account for item 1, it has been assumed that the emotion is a disturbance in the timing of thalamo-cortical firng-a dis- 


\section{Mechanisms of Emotional Response}

ruption of the phase sequence when the subject is awake, of the intrinsic organzzation of cerebral activity when he is asleep The disruption may be slight and brief, in this hypothesis, or extenstve and prolonged, it may be produced by a conflict of phase sequences, by a lack of sensory support for the phase sequence, or by metabolic changes.

Now the question 1s, how far a second assumption can account for items 2, 3, and 4, histed above. This assumption was made earlier (p 150), to account for the learning by a rat to choose a lighter door and avoid a darker one, in discrimination trainIng It is, in short, that a disruption of thalamo-cortical tuming tends to prevent the recurrence of the phase sequence that led up to that disruption on previous occasions, because of the cychical (anticipatory and recurrent) organization of the phase sequence. This is a mechanism of learning, and it is proposed now that it would account for the adaptive features of rage (emotional disturbance plus attack) or fear (the same thing plus avoidance), as learned behavior that is determined by the accompanying emotional disturbance. Particularly, it would account for the variability of the behavior and for the marked tendency of the emotional element itself to disappear as the onginally disturbing situation is repeated

Above and beyond the question of the intrinsic plausibility of the idea that flight, in fear, is a learned response (it may not be as implausible as it seems at first glance), is the apparent necessity of my first assumption, that emotional disturbance is a disruption of cerebral tuming If this is necessary, we must then find some way of accountung for the other aspects of emothonal behavior. Let us look again, then, at the causes of emotional disturbance, which may be arranged in three classes.

Class I. Take as a starting point the infant chimpanzee's fear of the strange It has already been seen that this is not innate, not a fear of what is totally strange, since the animal reared in darkness does not show it until vision in general has begun to bave some "meaning" for hum (unthl it arouses phase sequences). Consider further that a familar attendant $A$, weanng the equally famuliar coat $B$ of another attendant, may arouse the fear just as a complete stranger would. A causes no dusturbance, $B$ causes none, $A$ and $B$ together cause a violent emotional reaction It 
is this sort of fact that makes it necessary to suppose that two phase sequences may interfere with one another. The first class of emotional disturbance thus is one caused by a conflict (which may be extended to the disruptive effect of pain stumuls and other "unpleasant" sensory events).

Class II. Now a second case. It was for Iong heresy to suppose that there could be a fear of darkness. Darkness is a lack of stumulation (at least after the first few seconds in the dark), how can it arouse an emotional response? Fear of the dark is not likely to appear untl the age of three or later in human children (Jersild and Holmes, 1935) and so the skeptic who thought that his child developed the fear without cause could be answered by the old appeal to tgnorance. how can he be certain, whth the varied unsupervised expenences a chuld must have in a three-year period? However, the violent avoidance of solstude by young chimpanzees (Kohler, 1925) is at least as marked as the human fear of the dark, and can be shown to be due only to the lack of the perception of companions. This then implies a second class of emotional disturbance. fear of the dark, fear of solitude, fear aroused by loss of support, and the emotional changes observed by Ramsdell * following prolonged absence of the normal auditory stimulation. It would comprise also emotional disturbance due to contect with a dead body (which lacks warmth and the usual responsiveness); anger at the lack of social response in another person ("inattentuveness"), or a monkey's anger at not finding the particular knd of food reward he expected (Tinklepaugh, 1928), grief, homesickness, and so on.

- Personal communication from Dr. Donald A. Ramsdell, who was concerned to understand the high frequency of marked emotional changes which he observed in veterans following traumatic loss of hearing. He arranged expermentally to deafen bimself, effectively eluminating aurbome sounds for a period of three days The results are described by $D r$ Ramsdell as follows, particularly with regard to the question whether the deafiness would contribute to any neurotic or poychotic tendency

"In my experience there was a definite increase of irritability and a desire to ether withdraw from the field or, if held in the fleld, to "charge" mto It This would be comparable to being intated whth a frlend, and either withdrawing without saying anything or lothng hum have It." Such bohavior would not be charactenzed by ideas of persecution nor surplaion of the friend. However, you might say that the desire to 'charge' into a siturthon is such stuff as paranors 15 mede of, but for a true paranond idee of 
That a conflict of phase sequences, or a lack of sensory support for the phase sequence, should produce any extensive disruption in the tming of cortical action is not a logically unescapable deduction from the schema of neural action presented in Chapters 4 and 5 What the schematizing actually did was suggest the possiblity, as something that would account for a simular effect of two so dissimular causes and make the relationshp between perception and emotional disturbance intelligible. The idea then seemed to gain strong support when it drew attention to another set of facts that is usually forgotten in discussing emotional theory These facts concern the relationship of emotional disturbance to metabolic changes

Class III The thurd class of the causes of fear, rage, and so on, though it was actually arrived at last in formulating these 1deas, comes nearer than either of the others to being a necessary consequence of the neural schema To the earher discussion of the effects of chemical changes in the nutrient fluids that bathe the neural cell (p. 196), showing that a disturbance of timing must result, I need add only that a large intracranial tumor, compressing blood vessels and interfering with blood How, obviously could have sumlar effects, and also that asthma, or vascular disease, or an antigen acting to produce vascular spasm in intracranial vessels-any of these-might produce emotonal disturbance directly, by affecting the amount of oxygen in the blood or the amount of blood that is supplied to neural tussues Emotional changes are in fact frequently assocrated

persecution, the capacity for interpersonal relationship must be so weak that real suspicion can develop Assuming then interpersonal relationships to be secure, I would say that deafness is accompanied by a feeling of perplexity and disonentation analogous to the fear of the dark or fear of solitude Such a disonentation in one's socral mueu need not necessanly involve paranoid disturbances any more then being in the dark would induce one to hear footsteps at his back In my opinion, the feelings of personal inadequacies (impaiment of the body mage), iritability, and an exaggerated response to stumuli, and the wish to leave the field or, if held in the field, to "charge' into it, characterize the experfence of deafness"

I should like to draw attention also to Dr Ramsdell's suggestion that the emotional condition might lead to more than one overt pattern of response This is relovent to the later discussion of the way in which different "emotroms" might develop from the same primitive disturbance 
with all such conditions, and the one assumption that seems to provide a common ground for such varied sources of disturbance (Including not only the metabolic changes in Class III but also the perceptual conflucts and perceptual deficits of Classes I and II) is the assumption that emotional disturbance is in the first place a disruption of the tuming of neuronal activity in the cerebrum This also accounts durectly for the incoordinations of emotion Until some better guess is made, we must see what can be done with the assumption, and this imposes the burden of accounting for the integrated and coordmated aspects of emotional behavior (Leeper, 1948).

Now this coordunated part of the behavior, in the unpleasant emotions, has one constant function. It is always such as to tend to put an end to the original stimulation (in the pleasant emotions, of course, this tendency is reversed). Aggression, in rage, tends to change the urntating behavior of another animalby cowng, driving off, or kullung the annoyer Flight, in fear, tends to prevent or termmate noxious stmulation Avordenceturning the head away from an unpleasant sight, covering the ears, holding the nose, or withdrawing the hand, as well as actually running away-tends to stop the stumulation that arouses exther an irratronal fear of some inert object or the practically equivalent disgust Fawning may be a manifestation of fear as much as flight, and so may the desperate attack of a cornered anumal and each is a possible way of putting an end to the emotion-arousing behavior of another organism

Failure to respond, in shame, is a special case, in which one's own behavior is the emotion-provoking stumulus, the effect of the emotional disturbance is negative, preventing the recurrence of that behavior, but this is also in line with the present thesis, namely: All these effects may be acheved because strong emotlonal disturbance tends to prevent the repetition of any line of thought that leads up to $u$, and to eliminate the corresponding behavior. In some situations, a primitive, undifferentiated disturbance would develop into fear, where withdrawal is the only thing that ends the disturbance, in others, anger, when attack is the only effectuve behavior, in still others, either fear or anger might result, when either behavior would be effective, and which result happens would be determined by the anumal's past 
with all such conditions, and the one assumption that seems to provide a common ground for such varied sources of disturbance (Including not only the metabolic changes in Class III but also the perceptual conflucts and perceptual deficits of Classes I and II) is the assumption that emotional disturbance is in the first place a disruption of the tuming of neuronal activity in the cerebrum This also accounts durectly for the incoordinations of emotion Until some better guess is made, we must see what can be done with the assumption, and this imposes the burden of accounting for the integrated and coordmated aspects of emotional behavior (Leeper, 1948).

Now this coordunated part of the behavior, in the unpleasant emotions, has one constant function. It is always such as to tend to put an end to the original stimulation (in the pleasant emotions, of course, this tendency is reversed). Aggression, in rage, tends to change the urntating behavior of another animalby cowng, driving off, or kullung the annoyer Flight, in fear, tends to prevent or termmate noxious stmulation Avordenceturning the head away from an unpleasant sight, covering the ears, holding the nose, or withdrawing the hand, as well as actually running away-tends to stop the stumulation that arouses exther an irratronal fear of some inert object or the practically equivalent disgust Fawning may be a manifestation of fear as much as flight, and so may the desperate attack of a cornered anumal and each is a possible way of putting an end to the emotion-arousing behavior of another organism

Failure to respond, in shame, is a special case, in which one's own behavior is the emotion-provoking stumulus, the effect of the emotional disturbance is negative, preventing the recurrence of that behavior, but this is also in line with the present thesis, namely: All these effects may be acheved because strong emotlonal disturbance tends to prevent the repetition of any line of thought that leads up to $u$, and to eliminate the corresponding behavior. In some situations, a primitive, undifferentiated disturbance would develop into fear, where withdrawal is the only thing that ends the disturbance, in others, anger, when attack is the only effectuve behavior, in still others, either fear or anger might result, when either behavior would be effective, and which result happens would be determined by the anumal's past 
experience as well as by his own inherited peculianties But in some situations, and for some subjects, no behavior might be discovered that would abolish the emotional disturbance, and the effect of learning in this case might be to reunforce the visceral disturbance and the incoordinations of the skeletal musculature, and-above all-the disorganzation of the phase sequence, which is a disorganization of thought

This is the point at which neurosis and psychosis enter the theoretrcal structure they are (1) condibons of chronic emotional disturbance, or (2) ones in which emotional disturbance, in the past, has effected a lasting modufication of the thought process (see footnote, p. 259)

The first objection that might be made to such an approach is this. As the bypothesis has been presented so far, it mught suggest that aggression, in anger, appears only by default-only because other forms of behavior have been elmmated-and to common sense this is not reasonable Aggression appears to have a much more intumate and necessary relation to the emotonal disturbance of anger. And so with avoidance, in fear. My first answer is that this may be perfectly true, in the adult, even in the four-year-old But this proves nothing about the orggn of the relatronship. Emotonal disturbance and the aggression may seem inseparable to us for exactly the same reason that nodding the head and Yes are mseparable-in this culture: because the two have been thed in together by long practice and experience

It is instructive to observe just what happens in emotional learning, when we do have a chance to observe it. Bridges (cted by Jones, 1933) has shown that children's emotional patterns develop out of a primitive undifferentiated excitation. This fact may not be crucial, for the change may depend on physical maturation and not on a learning process But when chumpanzees are exposed for the first time to certan emotion-provokng situations, the first effect again appears not to be a spectic pattern of adaptation but a generalized exctation. When Alfalfa, one of the chimpanzees reared in darkness by Riesen (1947), was brought out into the light she did not show any emotonal disturbance at the sight of strangers, as I have already pointed out, the emotion requires a certain amount of visual experience 


\section{Emotional Disturbances}

before it occurs. When signs of the disturbance appeared some tume later, they seemed completely unspecific a raising of the general level of excitation, with signs of autonomic action and undurected movements. I have remarked elsewhere (Hebb, $1946 \mathrm{~b}$ ) on the lack of a consistent mode of response of some adult chimpanzees at the first exposure to a disguised person, wearing a mask and acting in an aggressive manner which they were not used to encountering in human beings, and also on the gradual emergence of a consistent mode of emotional response, but different in form for different animals

The implication of my hypothesis, then, is that adult introspection cannot be an argument against its validity, since the essential learning would be thoroughly established in early infancy.

Observe further the conditions in which the adaptive behavior of adult emotion occurs. Apart from the spinal reflexes aroused by noxious stimulation, a fully coordinated emotional response appears always to be a response to premontory cues, not in themselves disruptive, but associated with disruptive stimulation When the primitively disnuptive event occurs suddenly, without any warning, the response is never a smooth and economical cooperation of effector organs but is likely to involve startle, trembling, sweatung, vocalization, vascular change, and so on, and is then followed by a coordinated response which differs according to the subject's perception of the total situation If the adaptive behavor of fear (that 1s, avoidance) is indeed a response to events that are not drectly disruptive but premorattory cues associated with disruption, the behavior is by definition a learned response. It is then intelligible why there is usually less emotional activity at maturity. As experience increases, rage and fear would tend to disappear in the famuliar environment, for the utilization of premonitory cues, to avoid disturbance that is foreseen but not yet in exustence, would become more and more efficient The integrative part of emotional behavior and the disintegrative are inversely related: as one increases, the other decreases

The objection that we have just considered is that the connection between aggression and emotional disturbance, in rage, is not an accidental one-established merely by association or 


\section{Mechanisms of Emotional Response}

by the elimination of alternative modes of reaction The objectron may be taken further. It cannot really be a matter of chance whether fear or anger will result, oven in stuations in which ether avoidance or attack would have the same effect of changing a disruptive stimulation Characteristically, some situations evoke rage, others fear, and so on. The objection carnes weight, and must be considered I should say at once that I do not believe that it is sufficiently answered by what follows, and there are, with reasonable certainty, important constitutional factors determming the mode of emotional response which I cannot deal with. It is also possible that milder degrees of disruption innately tend to lead to aggression, stronger ones to avordance

In addition, however, factors of learning are present that should not be overlooked. One thing that determines whether fear or anger will result when the phase sequence is disrupted may be the particular phase sequence itself, and the effect of earleer learning in sumlar sstuations, which determines what alternative sequences are available to take over when the disrupted sequence leaves the field open. With this in mind, observe that anger is characteristically aroused by the behavior of others (ie, by soctal stumulation) when the behavior is not consistent with what one is proposing to do, when one expects resistance in havng the bekavior changed, and finally when one thunks one may be able to change it.

It is a humilating fact that one tends not to get angry at prize-fighters, or at the critucism of the higher-up whose praise or approval is important. This is far from being absolutely true, but it seems that anger is most easily aroused by opposition where one thinks one could preval by force. where the opponent ought to see that you can get your own way, and give in gracefully.

I believe it is also true that, the more authoritarian the regime in which a chld grows up, and the more frequent and severe the punishment he suffers for disobedience, the less likely he is, as an adult, to get angry at higher authority and the more likely to be angered by insubordination. If so, it means that anger does not occur in situations where disruptive stumulation has regularly broken up the phase sequence that determines aggres- 
sion, that it does occur where aggressive behavior has not usually been disrupted before. (That such evidence should have to be resorted to-I know of none apart from such folklore-is the best indication of a lack of scientific knowledge, on a very important topic in psychology, which is due to the difficulty of experimenting with any but the mildest of human emotions )

Now a final objection to the hypothesis that learning is what mainly dufferentiates the various emotions. Nothing has been said so far to provide for the exceptional vigor that an emotional response is apt to have. This I believe is the greatest defect in the hypothesis. Several things might be suggested to remedy it. One possibulity is that when dusorganization occurs it leaves transmussion units in the cerebrum ready to fire with any subsequent activity. These units would ordinarily be involved in the assembly action that is now prevented; they may then tend to be recrunted by whatever assemblies do act subsequently increasing the strength of the facilitation delivered to motor centers but also tending to make the motor response less precise (since the recruitment of extra cells would tend to change the frequency properties of the individual assembly). This might also be applied to the pleasurable activity, discussed in the preceding chapter, that depends on first arousing apprehension, then duspelling it. It may offer some cue also to the nature of laughter, whuch may be most efficiently aroused, perhaps, when there is a combination of phase sequences that produces a minor disturbance of tuming immediately followed by a further combenation of assemblies that have no conflict. There would still reman the fragments, so to speak, of assemblies that were disrupted, which tend to induce some motor activity. In some cases this may induce anger or fear or embarrassment, if the disruption is extensive; but in other cases a cortically controlled overactuvation of the breathing mechanisms may serve to pick up the fragments-which appears as laughter First a minor conflict, incongruty or apprehension, then a new perception of the situation that leaves no need of avoidance, nothing to be changed in the behavior of other persons or in the total situation, but still leaves a condition of excitation that must issue $\mathrm{m}$ one form of behavior or another. 


\section{- THE NATURE OF MENTAL ILLNESS}

It has already been proposed that mental illness consists etther of a chronic disturbance of time relations in the cerebrum, or a lastung distortion of the thought process from such a disturbance at an earlier time * This is a rather general proposition, and we must now consider the theoretical problems of mental illness in more detall. The general approach that has been proposed may have value only in helpung to mantain a pount of veew that is hard to maintain, and yet essentral The point of view is this neurosis or psychosis is a product netther of experience nor of constutution, but a joint product of both

Tradition, once more, is the villain of the piece In this field we are still dominated by the old dichotomy. is the illness mental or physical, functional or organic, and now, under new names, is it psychosomatic, or just somatic?

It may make sense to ask whether a breakdown is primarily experiental, implying that with an ordinary degree of strain it would not have occurred, or prumarily constitutional, implyng that the muldest of strains would have caused the breakdown, that the patient's ability to withstand strain was low. But this cannot give us two kinds of mental illness, any more than, in a completely parallel situation, there are two knds of tuberculosis

If there are not two kunds of mental lllness, we have no basis for dismussing the mental llness that goes with pellagra or some glandular dysfunction as not being a "true" psychosis; no basis for thinking of a special category of chronic emotional disturbance, of which experience is the sole cause, and psychotherapy in some form the sole method of cure. Logic and the whole clinical literature require that we remember the role of the con-

- This distenction is not further elaborated in the test It is primarily based on theoretical considerations, but it seerns to agree with the fact that the neurotic or paychotic frequently appears to have found a way of throking and acting that obviates emotional disturbance The illness is perbaps exther (1) a contanued emotional dusturbance, with anxiety or the luke, or (2) changes in thought (new phase sequences) that aro not characteristc of the majonty of the population but whuch avord some confluct that makes for major disruption of assembly or phase sequence. 
stitution in any mental illness, just as we must remember the role of experience. It is hard to avoid going to extremes when this topic is discussed, it is very hard to keep from thinking in terms of the old dichotomy, but I beheve we must keep a middle way. Untsl quite recently, the task of the psychologist and psychuatrist was to keep the medical world from thinking of mental illness as always due to some lesion of the central nervous system-a lesion that usually could not be found Now the pendulum has swang to the other extreme, and the medical world may be in some danger of overestumating the role of psychological causation in both mental illness and ilnesses that used to be called physical. The task is still to find a middle way

I believe there is no longer a danger that psychologxcal factors in illness will not be recognized there is hardly a popular journal in the western hemisphere that does not publush articles explaining how illness may be due to worry or to a desire to get out of some sort of trouble. In medical practice it may happen that fallure to make a definite diagnosis can automatically determme that the patient is psychoneurotic. This tendency has been protested agaunst by physicians concerned with the welfare of the patient (Bowman, 1946, Wlson and Rupp, 1947). But it also represents a mode of thinking by the psychologist or psychatrist humself that may huder the development of theory It is as much incumbent on the psychologist to demonstrate the limits of psychological influence in illness as to demonstrate its extent, as matters stand today, the first need for research in the field may be to ask what we do not know about psychological inHuence

Psychology's ignorance about a number of these matters is bad, but it is worse when we substitute dogmatism for scientufic knowledge We stull need an Ajax to stand up and defy the lightning and ask, What is the evience? when some authonty informs the public that believing in Santa Claus is bad for children, that comic books lead to psychological degeneracy, that asthma is due to a hidden mental Ilness, or that a child's bedwetting must be treated by psychoanalysus, and psychoanalysis alone, instead of the sumple and effective method of conditioning described by Mowrer and Mowrer (1938)

Let me then present four propositions for serious considera- 
tion, even though they may sound ndiculous: (1) that there is no separate class of mental llness caused by psychological factors alone-by what was seen and heard in childhood, (2) that we know little about "mental hygiene" or how to achieve it; (3) that it has not been shown that worry or the hke can by itself cause any bodily condition such as asthma or stomach ulcers, though it aggravates such conditions, and (4) that it has not been shown that any specialızed psychotherapy, such as psychoanalysis, has any special value in mental illness The first of these I think is logically inescapable, propositions 2, 3, and 4 may not be as absurd as they appear What I am talkng about is proof; one may know that psychoanalysis is of value and yet not bave the evidence in a form that would establish this as a scientific fact. Obvously, in a scientific argument one must accept the scientific canons, and puttng the endence in logical shape should ultumately lead to improved medical procedures as well as more precise theory.

If we are to improve therapy, or if prophylans is to be possible at all, we must improve theory first and know more exactly what leads to what. As long as such questons as those raised above are answered on a prion opinion alone, theory will get nowhere, for the experiments necessary to it will not be done. Evidently the current development of psychological ideas in medicine has a profound necessity behind $2 t$. let us see what kind of soltd factual evidence we need in order to keep this development on the ratis and to extend its influence, not only in the clinical but also in the social and educational fields.

\section{MENTAL ILLNESS AS A FUNCTION OF THE CONSTITUTION}

The first question rassed was whether there is any class of mental illness that is due to experience alone My point of vew here has already been stated that such a class does not exist, since any behavioral condition must be a function both of experience and of the constitution that does the expernencing We may take it, however, that the relative umportance of expenence and constitution may vary in mental iliness At one extreme, it might be assumed that there is no human constitution that will 
not break down when emotional disturbance is sufficiently severe and prolonged (Swank and Marchand, 1946), at the other, that metabolic changes alone may produce emotional disturbance that will persist as long as metabolic factors remain variable and unstable But the theory that has been developed indicates that the essental nature of the resulting condition may be the same in both cases There have been an increasing number of reports in the literature showing that traned diagnosticians have not been able to distingush mental illness precipitated by a physiological disturbance from the mental illness for which no such cause can be discovered, and reports as well showng that the characteriste "functional" disturbance is accompanied, to a more than chance extent, by constitutional deviations.

Typical neurotic or psychotic disorder may be found in brucellosis (Harns, 1944); in pancreatic disorder (Romano and Coon, 1942), in adrenal disorder (Allen and Broster, 1945, Greene, Paterson, and Plle, 1945); in perncious anemia (Bowman, 1935, Ferraro, Ariet, and English, 1945), m rheumatic brain disease, following rheumatic fever (Bruetsch, 1947), in hypochloremia (Saphir, 1945), in vitamin-B deficiencies (Willams, Mason, and Smith, 1939, cited by Jolluffe, Jolliffe, 1942, Matthews, 1938, Egaña et al, 1942, Spies, Anng, Gelpern, and Bean, 1938). Certain types of neurosis are associated to a more than chance degree with inherited vascular abnormality (Hauptmann, 1946, Cobb, Cohen, and Badal, 1946). Dewan and Owen (1945) have reported twelve from a much larger number of cases of schizophrenia, manic state, depression, psychoneurosis, and so on, which were so representative of the usual picture of mental illness that no physiological or pathological source was even suspected, but the causes eventually were found to include brain tumor, diabetes, uremia, and so on: in all cases, a major cause in the "organic" class

It may be impossible to distinguish neurosis following head injury from other neurosis (Gillespie, 1944). It is common, as soon as some such "organic" cause is found, to reclassify the illness, but such reports as those cited make it impossible to consider that there is any real psychological duscontumuty between organic and functional lls, as these terms are commonly used This first point is made both on logical grounds and on the basis 
of the actual clinical facts It is further reinforced by the factual evidence and discussion of Cobb (1944), Hoagland (1947), Landis (1947), and Bellak and Willson (1947).

Secondly, these considerations make it possible that a contributing organic cause will be found for all those cases that are now called purely functional The history of psychiatric knowledge shows one syndrome after another being removed from the functional class, as pathological and physıological knowledge has mcreased. There are still gaps in our knowledge of the complex chain of events in the nutrition of the neural cell, and it is still possible that all well-defined cases of mental disorder will eventually be found to depend, to a major extent, on a predisposing constitutional condition-hereditary or acquired

Consider for example Kallman's work, the man results of which have been summanzed by Garnson (1947). Among other statistics are the following among the relatives of patients with schizophrenia, the incidence of schizophrenia for brothers and sisters is 143 per cent, for fraternal twins, with the same constitutional similarity as any brother or sister, 147 per cent, for identical twins, 85.8 per cent For stepbrothers and sisters, growng up in the same home, the figure is 1.8 per cent. There is no apparent way in which these data can be reconciled with the idea that mental lllness is unrelated to heredity, that it is due alone to the way a chld is brought up One can argue that two chldren of different ages have different environments though they are brought up in the same home. An oldest child certaunly has a different social environment from that of a youngest child, and of course any one child in a family may have some bad expenence that another does not. This might explain why an ordinary sibling of the schizophrenic (born at a separate tume) is less likely to have schizophrenia than a twin. But-this dufference, predicted by the environmentalsst hypothesis, is not observed. siblings and fraternal twins have the same frequency, exactly as would be expected on the genetic hypothesis. And there seems to be no way at all of accounting for the difference between the figures of 14 per cent for fraternal twins, and 85 per cent for identical twins. As we shall see in the following chapter, the data from twin studies have not shown a great deal about 
the relation of heredity to the IQ, but they could hardly be much clearer as far as schizophrenza is concerned

There 2s, however, still the other side of the conn. Enghty-five per cent is not perfect concidence, it does not make heredity the sole determunant even of schizophrenia, and still less the sole determinant of some other mental ils A paper by Hobbs (1941) makes the point perfectly one of a pair of identical twras, brought up whth very strict moral standards, broke training as an adult and a psychotic episode followed The other twin showed no ill effects; heredity provided only a susceptrble constitution and did not alone determine that the psychosis would occur A similar case is referred to by Bellak and Willson (1047).

The same conclusion can be drawn from the very significant experiments of Pavlov (1928), Gantt (1944), Liddell (1938), Masserman (1943), and others on the "conditioned neurosis" or "expernmental neurosis" I have suggested (Hebb, 1947) that we should be cautous in identifyng this with a full-fledged neurosis in man, since the experimental neurosis is apparently specific to the situation in which it was established. But there can be no doubt that this work is very umportant for the understanding of cluncal neurosis, showing how anxiety (at the least) can be establsshed in a way that would not otherwise have been suspected, since no actual mjury or threat may be involved. Anxiety is one source of mental lllness, and Povlov showed that this chronce emotional disturbance can be created in some dogs much more readily than in others. apparently, a constitutional difference between dogs (Gantt, 1938) But here, again, there is cooperatson between constitution and experience, for it takes a very special expertence indeed to produce the emotronal breakdown in most anumals, even when they are definitely susceptsble.

\section{THE PRESENT SIGNIFICANCE OF MENTAL HYGIENE}

The second pount of our present ignorance about mental illness is the way in which early expenence may be related to a disorder at matunty. The mental-hygiene conception was estabhshed some years ago, when it was possible to think of mental illness as simply a set of bad emotional habits It seemed then 
that if one could avord such habits in childhood one could be sure of mental health at maturity, whatever the constitution one may have inhented

Now this may still be partly true, the unfortunate fact, however, is that we do not have any good basis for being sure how chldren should be brought up, in order to minumze their chences of getting into a state hospital The necessary experiments have not been done. What may be necessary to decide the questron is to bring up some chldren in one way, some in others, and see what leads to what One cannot assert that too much coddling produces mental lliness at maturity, or that too little does. For all we know, a lot of spankang, or unsympathetic parents, may only help to prepare the child for the trals of maturity It cannot be assumed that what produces emotional disturbance in the infant is bad-nor that it is good. the longtem effects may be the same as the short-term, unrelated, or opposed. One must make some decision when a practical problem has to be dealt with, and in these crrcumstances the only thing to do is make the best guess one can, but opmions should be presented as opinion, and not as scientifically established. fact

In such matters, our ignorance is virtually complete and vitally in aced of being replaced by definite knowledge. We do not know whether sexy comic books at the age of ten have any deletenous effect at twenty, we do not know that play with tóy guns and soldrers makes a war-loving nation, we do not know that juvenile delinquency, associated with broken homes, is due to the home envronment and not just as much to the inheritance of the same emotional unstablity that broke up the home

It has sometrmes seemed that answers to such questions might be found by anthropological study, when different primitive peoples treat their chldren differently, what are the effects at maturity? In one tube, for example, the chuldren are treated generously and the adults are found to be easy-going and friendly; in another, chld training is harsh, and the adults are found to be full of hosthities But, as Xlmeberg (1940, p 434) has pointed out, one cannot single out cause and effect here, any more than in the case of the broken home Heredity, in the two tribes, may differ signuficantly, producing dufferent adult 
personalitues, or some other variable such as the history of the tribe may be the source of the differences-and the reported facts may mean only that emotionally stable people treat their children well *

The mental-hygiene program first needs research, then more research, so that we can find out how to rear children to make them as healthy as possible, emotionally, at maturity. It is important to apply what knowledge we have now as best we can; it is equally important to remember how incomplete the knowledge is We are not now in a position to do more than apply the conceptions of one or other theory of mental illness, that becomes insufficient as soon as one tests it by facts I have already cited the cases of Alpha and Kambi, chimpanzees of the Yerkes colony, to show that, if early experience is a main cause of mental llness at maturity, we have no real information as to how it works The theory that has been presented here is much too vague, or general, to provide any useful gude in the practical problems of child education, it may be useful, however, if it leads to more of the obviously necessary research.

\section{PSYCHOSOMATICS AND PSYCHOTHERAPY}

The third and fourth points of ignorance that have been listed can be considered together. the questions are whether worry or the like can produce bodily lesions, and whether psychotherapy can cure mental illness Though the questions may sound ndiculous, I think it may be of some value to consider them seriously and ask oneself just what factual evidence one could cite to justify one's answers.

Just where can one look for the specific evidence on which to base the psychosomatic thesis? Clearly, it is not enough to show that neurotic disturbance and pathological changes in the body go together, $f$ or even that when the first clears up the second

- I $e$, reverse the statements above "In one tribe the adults are casygoing, and so chldren are treated well, w another the adults are aggressive, and children are treated harshly"-ag_one might expect

f Obviously, It is also not enough to show that emotion is accompanied by transient somatic changes in normal or psychopathic persons The very important studies of Wolf (1843) and Wolf and Wolff (1946), for example, 
does also-unless the recovery is due to psychotherapy. If some metabolic disorder contributed to the neurosis, it may have determmed the pathological changes also Then mental and physical difficulties would appear together, and disappear together. If one could show that asthma, or stomach ulcers, or skin lesions could be cured by talking to the patent-that is, by psychotherapy-then we would have the desired proof But, as we shall see shortly, there are great difficultues in the way of showing that psychotherapy is effective (chrefly, the difficulty of showing that it produces more cures than are accounted for by spontaneous remission). Despite its general acceptance, in which I concur, the hypothesis that psychological factors affect the recovery of the body from any physical illness has not been established scientfically. It may appear to the reader that it is not necessary to do so, but there must be much that should be found out about the psychological influence-in what conditions it operates best, what its limits are, and so on, and these things wil never be discovered until we start examming our assumptions, and start the slow, plodding process of bulding up a solid body of facts, separate from opinion, upon which the theory of treatment can be firmly established

Not all cases in which a "psychosomatic" relationship is seen are ones in which the onginal trouble was emotional. The following case of early asthma offered an exceptional opportunity for separatmg cause and effect. It is rare that one can have an effective control of asthma, and banish it or not, at will-but when this is possible, new light is cast on the disease.

A chld of twenty-one months developed acute, severe attacks of asthma after having had a head cold some months earher and a persistent nasal infection The attacks became less frequent several months later, during the summer, but recurred with increasing severity and frequency as winter set in Eventually it do not show that chronic gastric and cardiac lesions result from emotsonal dusturbance alone, whthout contribution from a preceding physiological disorder, although, if it is assumed that "normal" emotion has such effects, these studies help to show us how the lesions are establshed It should bo clear that what is discussed in the text is the question of scientific proof, not of reasonable belief Belef in the psychosomatlc thesis is reasonable, perhaps inevitable, but the very importance and value of that thesss requre that its foundations be inspected and tested ngorously-and pernodically 
appeared that a running nose preceded each attack, and it occurred to the physician in charge that a nasal infection might be the source of the allergen He found a beavy chronic baclllary infection, and recommended chemotherapy. The results were dramatic. The asthma disappeared completely, and a remarkable change took place in the chuld's behavior. Dunng an attack, the child had been almost completely incapacitated, but between attacks, there had been no difficulty $m$ breatbing and the child appeared quite normal-except that she was always emotionally unstable, easily giving way to tears and being rather hard to deal with. Actually, this behavior had developed so gradually during the penod of illness, and was so constant, that the parents had not realized that there was any connection between the emotional picture and the intermittent asthma. Now the patient became a different chuld.

Two further observatons made the interpretation of these facts even clearer. It was expected that the allergic sensituvity would gradually disappear as the chuld grew older, so on two subsequent occasions when a nasal unfection began the treatment was omitted at first, in the hope that asthma would not develop On each occasion the same sequence of events was observed. The first symptom of disturbance was curculatory-the child's hands and feet became very cold. This was followed by the same emotional disturbance that had been chronic earlier, at least a day before the first symptoms of difficulty in breathing, and finally, a definite asthma appeared. This and the emotional changes were then promptly (in six to twelve hours) banshed by chemotherapy Such a sequence of events can only mean that the allergen affected the vascular system first-the cold hands and feet were not due to lung congestion and decrease of oxygen in the blood stream On one of the two occastons on which treatment was witheld, moreover, a placebo was used This served as a control of the effects of suggestion, showing that it was in fact the actron of the drug which, when it was actually used, produced the recovery from asthma and the disappearance of whining, crying, and the need of contunual attenhon Only one conclusion seems possible. that the allergen acted more or less durectly on the vasculature, including the cerebral vasculature, or on the central nervous system, thus caus- 
appeared that a running nose preceded each attack, and it occurred to the physician in charge that a nasal infection might be the source of the allergen He found a beavy chronic baclllary infection, and recommended chemotherapy. The results were dramatic. The asthma disappeared completely, and a remarkable change took place in the chuld's behavior. Dunng an attack, the child had been almost completely incapacitated, but between attacks, there had been no difficulty $m$ breatbing and the child appeared quite normal-except that she was always emotionally unstable, easily giving way to tears and being rather hard to deal with. Actually, this behavior had developed so gradually during the penod of illness, and was so constant, that the parents had not realized that there was any connection between the emotional picture and the intermittent asthma. Now the patient became a different chuld.

Two further observatons made the interpretation of these facts even clearer. It was expected that the allergic sensituvity would gradually disappear as the chuld grew older, so on two subsequent occasions when a nasal unfection began the treatment was omitted at first, in the hope that asthma would not develop On each occasion the same sequence of events was observed. The first symptom of disturbance was curculatory-the child's hands and feet became very cold. This was followed by the same emotional disturbance that had been chronic earlier, at least a day before the first symptoms of difficulty in breathing, and finally, a definite asthma appeared. This and the emotional changes were then promptly (in six to twelve hours) banshed by chemotherapy Such a sequence of events can only mean that the allergen affected the vascular system first-the cold hands and feet were not due to lung congestion and decrease of oxygen in the blood stream On one of the two occastons on which treatment was witheld, moreover, a placebo was used This served as a control of the effects of suggestion, showing that it was in fact the actron of the drug which, when it was actually used, produced the recovery from asthma and the disappearance of whining, crying, and the need of contunual attenhon Only one conclusion seems possible. that the allergen acted more or less durectly on the vasculature, including the cerebral vasculature, or on the central nervous system, thus caus- 
ing both emotional and physical symptoms. Ordmarly, with multtudinous sensituvities and no possibility of such cleancut "experimental" manupulation of allergens, cause and effect cannot be separated. The case described might bave been a typical psychosomatic picture: first emotional disturbance is evident, then physical symptoms follow. So the first must cause the second But in this case such an interpretation is not possible

Because asthma and emotional disturbances go together, or even because an emotional disturbance can precipitate an attack of asthma, it does not follow exther that emoton caused the onginal difficulty, or that psychotherapy can do more than allevate an illness that may have quite a different basis It is still possible that some cases of asthma, or some other boduly llness, are psychogenic and can be cured by psychotherapy, but evidence of this has not yet been provided in a form in which it can be evaluated. Future research may well show that the causal relationships in the case I have described are the exception and not the rule (ie, may show that allergies are usually psychogenic) But this research has not yet been done.

It is amplied throughout this discussion that, if psychotherapy can be shown to be an effective treatment of asthma or mental illness, tho argument above would be reversed. It seems generally beheved that the validity of psychoanalytic theory, for example, is well established by the fact that it has led to curng mental illnesses that would not otherwise have been cured.

If one looks for the evidence, however, one cannot find it. There is no meanung in the fact that a particular patient was psychoanalyzed, and recovered. We do not know that he would not have recovered without the treatment.

The point is clear when it is recalled how defficult it was to show that the insulm-shock method had any value. Psychratrasts and physicuans in general were skeptical Why? Because some not-very-exactly-determined proportion of psychotic patients recover without treatment-perhaps 30,35 , or $\mathbf{4 0}$ per cent. The figure varies whdely from one senes of patients to another; and it took five to ten years of work, by a large number of investrgators, to finally establsh the fact that insulin therapy signifcantly increases the proportion of patients that recover from schuzophrenua. A few patrents recovering after lobotomy would 
mean nothing. Freeman and Watts' (1942) trumph was in being able to report not only 63 per cent good results in a long sernes of chronic cases of mental illness, but much higher percentages with certain types of case. These figures had an immediate meaning, since the research on shock treatments had already made known what the spontaneous recovery rate would be, roughly, in such cases No such data, so far as I can discover, have ever been provided as a test of any form of psychotherapythat 1s, the frequency of recovery in an adequately chosen, untreated control group is not avalable to compare with the frequency after psychotherapy is applied The one exception to this statement is the paper by Denker (1946), and his conclusions do not show any specral value in formal psychotherapy.

Denker's paper is based on admittedly unsatisfactory data, and obviously is not a final answer to the question it has raised, yet it stands as a very umportant paper, simply because it is the only one, apparently, that has attempted an essentral task.

The data reported by Denker are the rates of recovery in a senes of 500 patients with severe incapacitating neurotic dsorder, treated by general practstioners throughout the United States. These patients were all receiving disability payments from an insurance company -a fact that might delay recovery, at least as compared with the recovery of patients who were paying for treatment. At the end of five years, the recovery rate was 90 per cent. Denker also reviewed the reported recovery rates under more specialuzed treatment. The average rate of recovery under psychoanalysis is roughly 62 per cent, under psychiatnc treatment (which may have included psychoanalysis), roughly 70 per cent. These rates are markedly lower than that obtained by the general practitioner However, if we assume that the results by speculuzed treatment are for a shorter penod, perhaps two years, we find that the general practztioner's results are about the same. 72 per cent. The conclusion is that rest, sedation when necessary, reassurance, and time are what produce recovery from neurotic disorder-when recovery occurs.

Obviously, this conclusion is not final. We do not know that the patients treated by psychoanalyst, psychiatrist, and general practutioner, in Denker's analysis, were really comparable (however, the one respect in which it is certain that they were not 
comparable-the economic factor-is such as to strengthen Denker's argument) Denker's conclusions may be quite wrong; but the real point is that his are the only data ${ }^{\circ}$ we have by which to evaluate psychotherapy.

Such a result cries for research, if nothing else has induced the psychoanalyst to give us some control data, some evidence for the validity of his theory and treatment of mental llness, this should do so In the meantime, there is no body of fact to show that psychotherapy is valuable, there are no data to prove that psychotherapy can cure lesions of the gastrointestinal tract, dermatits, and so on; and none to prove that there is a class of "functional" mental illness that is produced by emotional disturbance alone and susceptible of cure only by talkng to the pattent or allowing the patient to talk I think any psychologist (or physician) must believe that psychotherapy is important, as an adjunct of other treatment, and even by itself, but if we are to increase its efficrency, we must leam more about it, and the first thing to establish is the definite fact that it works at all.

\section{SHOCK TREATMENT AND LOBOTOMY}

The final concern of this chapter is a rationale for the shock and surgical treatments that revolutionized the field of psychiatry.

Lobotomy, a surgical operation that severs most of the connections between the frontal pole and the rest of the bram, and

- An earter discussion by Landis, however, based in part on the same data, has clearly led to the same conclusion and must be regarded as reinforcing Denker's conclussons (C Landus, A statustical evaluation of psychotherapeutic methods, in L E Finsie, Concepts and problems of psychotherapy, New York Columbia Unv Press, 1937, pp 155-169) Landis first determined the reported rate of recovery from psychoneurosis, apart from spectalized methods of treatment, as varying around a central figure

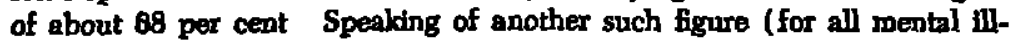
nesses), he went on to say that, although the figures given were not stnctly speaking those for spontaneous remission, "still any therapeutac method must show an appreciably greater stze than thes to be sertously considered." Finally, he reported a figure of $\mathbf{5 8}$ per cent for psychoanalytic treatment of the reuroses The inference, not made explicit by Landus, is the same as Denker's. 
electroshock treatment, in which an electric current passed through the skull induces a brief convulsion, both appear to work best with patients suffering from emotional depression, and both frequently have the effect of banishing the depression. In trying to understand how a cure is effected, the first question might be, What do such different procedures have in common?

The surgical treatment (Freeman and Watts, 1942, 1946) severs a large number of connecting fibers in the brain and leads to retrograde degeneration of a particular nucleus in the thalamus (the nucleus medialls dorsalss). A number of invest1gators have expressed the opinion that it is the loss of this tissue that decreases the strength of the patient's emotion and allows him to recover But no such gross destruction of tissue results from the passage of the electric current through the head Is there any other effect of the surgery that might produce emothonal changes?

A suggestion comes from the study of frontal lobe removals A comparatively common operation, prefrontal lobectomy, is usually done so that it severs the same connections that are severed in lobotomy-but severs all other connections of the same area, and actually removes the tussue In these operations, consequently, the medial dorsal nucleus of the thalamus must degenerate. But the knd of emotional picture drawn by Freeman and Watts (1942), as representing the results of frontal Jobotomy, is sometmes definitely not present (Nichols and Hunt, 1940, Mixter, Tillotson, and Wies, 1941, Hebb and Penfield, 1940) On the basss of this fact it was suggested (Hebb, 1945b) that the effect of lobotomy might be due not to a sumple loss of some function resident in the frontal lobes but to a physiological disturbance set up in that area by an operation that cannot belp but be scar-producing This would account for the fact, also, that the recovery from depression, in lobotomy, is reversiblealthough the degeneration of the thalamo-frontal connections and of the medial dorsal nucleus is not reversible

A physiological disturbance in the cerebrum is usually detected in the electroencephalogram, or EEG, by the presence of large potentials (hypersynchrony). My proposal, therefore, amounted to this. that in those patients that recover from depression following lobotomy the EEG will show a hypersyn- 
chrony, and that if relapse occurs the hypersynchrony will have disappeared or decreased Cohn (1945) has reported persistent disturbances in the EEG "in most subjects" of Freeman and Watts' senes, and delta waves in ten out of fifteen in one group of patients exammed four to six years after operation This is almost exactly the reported frequency (63 per cent) of chronic recovery from depression. Unfortunately, I have not been able to discover whether those with "bad" EEG's were ones with good clinical results (i $e$, lastung recovery), as my hypothesis would predict.

However, a durect confirmation of the hypothesss, up to a certain pount, has been provided by Hoagland, Malamud, Kaufman, and Puncus (1946) They report a lowered mcidence of fast waves (14 per second or faster) in patients showing remission from depression following electroshock treatment, and the return of fast waves at the time of relapse. This is durectly in the line with the hypothesis, although it may be a strain on the tern "hypersynchrony" to say that it may appear in a statistical decrease of fast waves, the change is actually in the same durection as if delta waves had been observed.

If the hypothesis should be further confinned, the effects of electroshock and lobotomy both could be understood on the following basis. It has been proposed here that emotional disturbance consists of a disorganized firing of cerebral neurons. The establishment of hypersynchrony, due to any cause, would provide a pacemaker, as it were, that would tend to pick up any neurons that are not ncorporated in assembly activities and so substitute the "intrinsic" organization of cortical activity for the diffuse organization of the normal adult wakng state The presence of the hypersynchrony would tend to interfere with the assembly and to lower intellectual competence at the same tume that it decreases emotional disturbance. Further, this would account for the lowered pain sensitivity, and the value of lobotomy in cases of intractable pain (Watts and Freeman, 1946). It will be recalled that pain was treated (in Chapter 8 ) as the equivalent of a local emotronal disturbance, in the somesthetic system, and so can be regarded as being affected in the same way by a focus of hypersynchrony. 
274 Emotional Disturbances

It is not necessarily implred that scar formation is what produces the hypersynchrony in lobotomy The effect of deafferenting an extensive cortical region would be to permit it to go into spontaneous hypersynchrony (Adrian, 1934). This, or this plus any effect of scar formation, may be the source of the recovery from mental depression. 


\section{The Growth and Decline of Intelligence}

This discussion began onginally, in Chapter 1, with the puzzle of the high intelligence-test scores that are sometmes found after a surgeon has destroyed a large part of the human brain A tentative explanation was suggested, that these scores are due to a conceptual development which bram damage does not easily reverse The chapters that followed have tried to make this intelligible physiologically (besides showing what the implications are for other problems) "Conceptual development," "insight," "thought," and so on, have been given a physiological meaning, and a possible basis has been provided for seeing how capacities could be retained, once developed, despite bram injury which if it had occurred in infancy would have prevented the development. This chapter returns to the onginal problem of the effects of brain operations on intelligence It presents an interpretation which fundamentally concerns the nature of intelligence, its normal development, and its later decline with old age

This chapter will be concerned also with certan problems of method Clnical research on intelligence has difficulties as a blackberry-bush bas thorns, and yet is an important source of information for the psychologist, and can be expected to become more important If the difficulties are to be surmounted, we must know what they are These matters of method will be taken up first.

\section{IDENTIFYING INTELLECTUAL DETERTORATION}

We feel, most of us, that we can make a reasonably good estimate of another's intellectual ability by conversation with him; 


\section{The Growth and Decline of Intelligence}

and we are apt to feel, too, that we have in general a faurly good xdea of what sort of problem can be solved by the healthy, "average," "normal" people in our own communities Both convctions are frequently misleading. The first point on method is that the detection of intellectual defect, following bram injury, requires the test procedure and (2n some form or other) the use of a control group.

Ordinarily, the person who is clear in conversation, quick to understand what is said to him, and coherent in his reply, is "Intelligent": that is, conversational ablity is correlated with other abulities, and is not a bad basis of prediction. But these correlations sometumes break down with pathological changes in the bram, and then the prediction may be false. Also, one is apt to judge capacity by the patient's alertness and manner, apart from what he says The physician is famliar with deterioration in the form of unresponsiveness, apathy, slowness, or unconstancy of purpose. Accordingly, when such signs are absent the physician may feel certain that intelligence is not impaired

It is reasonably sure that this has been a major source of the disagreement about utellectual impairment in aphasia Because the aphasic always tries to respond, is fully attentive, and gives the impression of being quite clear mentally, it is easy to conclude that be has lost nothmg but the power of verbal recogntion and expression. There are cases on record in which it has been "proved" that aphasia does not affect intelligence, on the ground that the patient nodded and smuled when the physician greeted hum, that he knew whether it was mornung or afternoon, or that he was able to let an attendant know when he needed the bedpan.

Tests, on the other hand, show that the aphasic usually cannot fit blocks together to make a simple pattern, that he does not recognize absurdities in pictures, cannot rake a simple drawing-even though be clearly knows what he is to do In such performances the aphasic is almost certain to be at a much lower level than a normal individual of the same background and 
original ablity (Weisenburg and McBnde, 1935, Hebb, 1942a). The aphasic patient is apt to have trouble in finding his way about, even in a famulrar regron, and to bave trouble in manipulating simple mechanical contrivances There is no sense in arguing over terminology, and each writer can define that equivocal term "intelligence" to sut himself (though one might expect some consistency in tts subsequent use), but because the aphasic is alert, and persistent in trying to discover what 2 and 2 add up to, or to discover what is missing in a picture of a dog with three legs, it does not follow that he has lost only the capacity for communication. There is also a change in what would usually be called intelligence.

To detect deterioration, one cannot depend on mpression alone but must also use test methods, and this requires a normal control group. It is most dangerous to assume knowledge of what the "average" person knows and can do. Let me give some examples, based on data obtaned in the standardization of some adult tests (Hebb, 1942b, Hebb and Morton, 1943) Somewhere in the neighborhood of half the adult English-speaking population cannot complete the analogy foot is to ankle as hand is to what? or under . over-down $?$ (though almost everyone can do dog . bark-cat - ?). About half the adult population think that priceless means "of no value", nearly the same number are unaware that brunette refers to a dark complexion, and many think it a sysonym for "blonde"-meaning, any lively young woman. Fugure 19 shows some simple problems-apparently simple-that cannot be solved by some fraction, between 10 and 25 per cent, of the adult population (Hebb, 1945b).

The subjects in these tests were able to earn a living and conduct their own affaurs with ordinary prudence, and would not be classed as anything but normal in the clinic. If such a person happened to have a brain injury, and the examiner did not know what other persons of the same class would do with such tests, he would be bound to conclude that the brain injury was the reason for the subject's rncompetence. No matter how sumple one's tests may seem to be, the brain-injured patent's performance must be interpreted in the light of the scores of a normal control group The professional man's idea of the average untel- 


\section{The Growth and Decline of Intelligence}

ligeace is colored by his preoccupation with the abstract and academic, and by the fact that his intumacy is mainly with others in the professional community whose preoccupations, interests, and abulities are of the same kind as his own.

How is a normal control group to be chosen? Despite some recent discussion making this an esoteric question, almost impos-
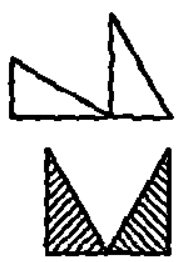
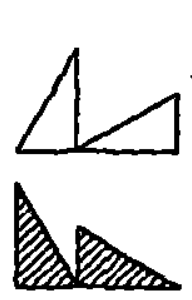

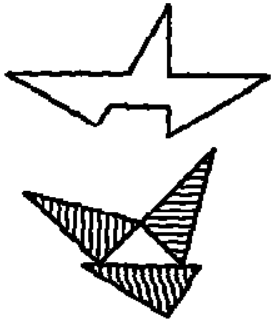

Frcure 19 Problems from a performance teat (top line) and attempted soluteons (bottom line) by a person not in any way classed as feeblemrnded A pattern in cardboard was placed before the subject, and left there while he tried to duplicate it, using two or more identical wooden trangles. Each of the hatched diagrams shows a subject's placing of the blocks in an attempt to duplicate the pattern ummediately above (by B G, female, 30 years of age, a "nomal control subject" convalescent after appendicitts) A faurly large proportion of the adult population will make such exrors, and have difficulty in seeing how to change the placung of the blocks when told that the solution is not correct

sible to understand, the desiderata are comparatively simple There might be no need of a control group if one could compare the patient's scores after bram operation with his scores before anything was wrong with his bram, all one can get, however, is a pre- and post-operative comparison, which must be a different matter. The condition for which he is being operated on must itself affect his scores, and in fact the operation quite often . causes a ruse in score. The purpose of the control group, then, is to let us know as far as possible what the brain-operated patient would have done if his brain had remanned healthy and undamaged This tells us what to look for in a control group. The object is to find persons who are like the patients being studied in every way that, as far as one knows, affects intelligence-test 
scores, except in the one respect, that the normal control subjects have undamaged brauns.

Now test scores are related to education, social background, , and occupation-a concatenation that can be summarized as the factor of sophistication Scores are also affected by age, nising, to a peak in the teens and declining (for almost all tests) after the twenties These are the correlates of intelligence in normal persons, as far as they are known at present, if they are controlled, one can estumate, by comparing climical and control groups, the degree and kind of umparment that results from brain damage, but if they are not controlled this is not possible. It is not possible to show that bram damage affects intelligence, by the use of a control group that is made up of hospital employees, or college students, or clerical workers, when the clinical group includes truckdnvers, farmers, and unsklled laborerspersons who are apt to have been long away from clerical work, with a lower average level of education. It is not possible to compare a man who went to high school ten years ago with a student now in high school. If the clinical subjects are older adults, or less sophisticated, they are bound to have lower scores than the normal subjects.

These considerations are what should determine the nature of the normal control group. One must then go farther, and use clinical controls, if one wishes to know not only that a certain kind of injury produces intellectual defects but also whether it produces more defect than some other knd of mjury. If one wanted to show that frontal-lobe damage is more serious than damage elsewhere in the brain, one would use two groups, a frontal-lobe group and a parietal, temporal, occipital group But note this. everything that was said above about making the normal group equvalent to the clinical group is equally necessary in the comparison of two clinical groups. They must be the same in age, in sophistication, and, in addition, in the size and type of lesion

Halstead (1947) has cnticized Rylander (1939) on the ground that his normal control group was not a clinical control group, and me (Hebb, 1945b) for being taken in by Rylander Actually, Rylander's normal control group was an excellent one, the only good one that has been used in the study of the frontal 


\section{The Growth and Decline of Intelligence}

lobe. His first object was to show that frontal-lobe damage produces intellectual defects, and he showed this clearly (Whether the defects are greater than with parietal lesions, or whether they are due to the surgical lesion or to an accompanying dysfunction, would be another question.) Halstead's control data, both normal and clincal, do not meet the elementary requirements set forth above his normal group was younger (the average was 26 years) than ether of his clinical groups and had a high proportion of persons with clencal and academic sophistication. His parietal, temporal, occipital group (33 years) was younger than his frontal-lobe group (40 years), the data supplied as to the relative extent of frontal and nonfrontal bram damage (drrect or indirect) are insufficient, and so too are the data as to occupation or sophistication (the designation "housewife" should be supplemented either by educational level or husband's occupation)

For some reason, frontal-lobe patients in the clinic sometimes tend to be older than others and may have a larger total destruction when the effects of pressure in the rest of the bran are considered Thus may be because the first signs of a frontal-lobe tumor are less specific than those of a tumor elsewhere, so the patient does not seek treatment as promptly. Frontal tumors tend also to produce bulateral damage of corresponding structures, by pressure, more than other tumors do. Consequently, it is very difficult indeed to find cases in which there is, for example, comparable damage of one frontal and one temporal l'obe, or of both frontal and both temporal lobes.

There is notheng mystenous about the logic of the controlgroup method If one wants to estumate the pre-operative intelligence of a group of barbers with frontal-lobe injury, or of highschool teachers, one would look for a group of barbers or highschool teachers of about/the same age and education, and preferably from the same part of the country, since occupatonal standards vary. The trouble of course is that $\operatorname{man}$ is a suspicious, sensitive, and uncooperative anumal who objects to having his intelligence tested and is not usually interested in helping a scientific mnestigation at such a price. To obtan test data for a good sample of the general population, even if only a sample 


\section{Anatomical and Pathological Data}

of fifty or sixty subjects, is a very lange undertaking (Weisenburg, Roe, and McBride, 1936).

The problem of normal control data has been reviewed elsewhere (Hebb, 1945b) The conclusions arnved at can be stated again Only two studies have utilized an adequate normal control group in psychological study of the effect of brain damage Rylander (1939) and Wessenburg and McBnde (1935). These two consequently are of outstanding importance (though they are defective in not having adequate anatomical data, and one cannot be quite sure that the defects were due to a simple loss of tassue). Rylander's norms, of course, sunce they are for another culture, are not applicable directly to English-speaking patents, but Weisenburg, Roe, and McBride's (1936) careful, though apparently little-known, analysis of intelligence in a representative group of adult English-speakung subjects still stands as the best basis for the study of intellectual defect. With cauton, their data may still be used if one cannot undertake the tremendous task of obtaining new normal-control data for each new study of brain damage

Dr. Morton and I have also supplemented their test battery with some new tests designed especially for adult use (Hebb, 1942b, Hebb and Morton, 1943). " Such norms, however, become less and less accurate with the passage of tume, and as more individual tests, of the homogeneous type, become avalable it would be desirable for sòmeone to attempt a new standardization of a battery of homogeneous tests, each one measuring a single kind of ability and so making it more possible to analyze the intelloctual changes that occur in cases of bram damage.

\section{ANATOMICAL AND PATHOLOGICAL DATA}

The second major aspect of the problem of method in such studies is the difficulty of gettung satisfactory anatomical and physiological data In an anumal study, the only data one trusts

- A recent restandardization of one of these tests, in a short form that is more convenient for clinical use, has been done by W A. Hunt, E C French, and I N Mensh An abbreviated form of the McGll Verbal Situation Test, $J$ Cln Psychol, 1948, 4, 406-411 


\section{The Growth and Decline of Intelligence}

concerning the locus and extent of lesion are obtamed by klling the animal and removing the brain for anatomical study. Human patients, after bram operation, have a regrettable way of living on indefinitely-instead of first making à gōod recovery, to permit thorough testmg, instructung ther relatives to permit an autopsy, and then dying of pneumonia

So one is obliged to make the best guess one can, instead of having exact data It is not possible at operation to know exactly what tissue is destroyed, particularly when the operation is for a tumor which displaces the bram-as tumors must-and in_dusplacing it_ys_more_than_likely_to_produce-a.duffuse-damage throughout parts that may be at somedistance from the scene of operation. In operations for tumor, actually, one is practcally always dealing with two kunds of lesion. the clean surgical lesion that can often be accurately described, and the diffuse, irregular lesions that result from pressure and have no clearcut boundary. Bram operations seemed to open a new era in the study of the localization of function, as they became common about 1920. Why? Because the effect of pathological lesions is almost impossible to interpret. One cannot say where the diffuse lesion begins and where it ends, attempting to interpret such data, between 1870 and 1920, had led to an endless senes of arguments. But this dufficulty apphes equally to interpretung the effect of a bram operation plus a pathological lesion, so in most cases of tumor the fact that the brain has been operated on does not actually make the case suitable for the localization of function.

Such a point would mean little if the effect of the added pathological lesion were slight. But it has been discovered that a comparatively small region of pathological change, producing hypersynchrony, may have a much more deteriorating effect on behavior than a complete loss of the tissue concerned An idea of this sort was proposed by Jefferson (1997) and Stookey, Scarf, and Tettelbaum (1941). A drrect demonstration on the point has been provided in two cases in which the removal of frontal-lobe tissue, in an area of scarring, changed a marked detenoration into behavior undistingushable from the normal (Hebb and Penfield, 1940, Mixter, Tillotson, and Wies, 1941). A region in which the blood supply is interfered with, but not 


\section{Anatomical and Pathological Data}

entirely shut off, usually shows some loss of cells and a number of remaining cells whose staining properties are changed. This indicates a change in the chemical properties of the cell, which in turn umplies a change in frequency properties and obvously may account for the existence of a hypersynchrony which interferes with the functioning of the cell-assembly A focus of hypersynchrony must act as a pacemaker that tends to wean transmission units away from the assembly When hypersynchrony is not great, it would allow some assemblies to function (particularly those that are long establsshed p 197) but would tend to interfere with recent memory, decrease responsiveness, and interfere with complex intellectual activities. When it is mgre extensive, it would prevent all higher functions.

$\checkmark$ Hypersynchrony thus extends the influence of a pathological lesion beyond the borders of the lesion itself and may make it impossible to draw any conclusion about the localization of the functions that are affected after a frontal pole, for example, has been removed When one is interested in an attempt at localization of function, the question must always be asked whether the sequelae of operation are due (1) to the surgical removal, (2) to permanent damage in the remaining parts of the brain, (3) to the transient physiological disturbance that always occurs along the margins of the surgical lesion, that may take from one to six months to subside, and that, since it is reversible, has deceived more than one worker into thrnkung that recovery is an evidence of ncarious functioning, or (4) to a chromic physiological disturbance, from scar formation or from diffuse pathological lesions throughout the rest of the brain If a loss of function is due to the surgical lesion, the function depends on the tissue that was removed, if due to dysfunction, it depends on tussue that still remains. All this makes the question of localization complex indeed. It explains why we are hittle farther forward in the localtzation of function, in the human brain, than Broca and Jackson were-the "experments of nature" are poorly designed, and produce disturbance, as well as a sumple loss, of function.

The EEG may be a relatively crude undex of what is going on in the cerebrum, with no durect relation to the firng of the individual cell, but it is a practical index of the physiological 


\section{The Growth and Decline of Intelligence}

status of the different parts of the brain, and as such it has the greatest importance in any attempt to study localuzation of function from a patient's behavior after recovery from brain operation. For such a purpose, electroencephalographic_report is not merely desirable but essential. With it, one has at least some chance of knowing when defects of behavior can be attributed to a surgical lesion and not to a pathological dysfunction instead.

Anumal experuments, anatomical studies, and studies in which the brain of the fully conscious human patient is stumulated electrically have clearly localized a number of sensory and motor areas But, because of the difficulties of method that have just been considered, about the only localization of a higher function that has so far been achreved is that of the so-called speech area the "midale regions of one hemisphere, usually the "dommant" one (the left hemisphere in a right-handed person), appear to have some sort of executive and organvang role that is essentral to speech and some other higher functions. No other localization of function in the human cerebrum has been established. I'äm aware that this statement is not in accord with much of the literature, but cribcal evaluation of the evidence presented in support of the various clams concerning localization permits no other conclusion, so far as I can see.

A paper by Klebanoff (1945), very useful as a review and bibliography of most of the psychological work in this field up to 1941, did not sufficiently consider the tww problems of normal control data, and enatomical and pathological data Fallure to solve one or both of these problems tends to invalidate the papers that try to prove that some higher function is resident in some particular part of the human cortex, in the work reviewedby Klèbañof. "If one considers the normal control data used in thiese studies, or the anatomical data, whth the same critical eye that would be turned at once on an animal expenment, one will find none of them justrymg more than an opmion, a guess, concernung the relation of higher functions to the cerebrum

Even with regard to the speech area, it is none too clear either what the boundaries are, or that a limited surgical lesion in any part of the region will disturb speech Aphasia may be produced only by a hypersyachrony whose focus is strategrally 


\section{Anatomical and Pathological Data}

placed here, in a position to disturb function throughout the whole hemusphere We bave no evidence on thrs, for the surgeon naturally declines to touch thus area if he can help doing so, if he must, he disturbs as hittle of it as possible.

However, the case of left hemidecortication reported by Zollinger (1935) shows that a clean surgical extrupation can produce aphasia. Again, antenor lesions in the speech area may produce more diffculty in speaking and wnting, postenor lesions more dufficulty in understanding speech and printed matter, but no real anatomical evidence to this effect seerns to exist, and Weisenburg and McBride's (1935) cautious distmction between a predominantly expressive and a predominantly receptuve aphasia should not be extended into a sharp dichotomy of "motor" and "sensory" aphasias, since it seems highly likely that both aspects of the disorder are always present

A number of clinical writers have disagreed with this conclusion, arrived at by Wersenburg and McBnde, Head, and others, but they seem never to have establushed satzsfactory evidence An important paper by Kennedy and Wolf (1936), for example, reports a "pure alexia" and a pure motor aphasia, with no other involvement. But the case reports are typically mcomplete to show that alexia is aphasic, it should be shown that there is sufficrent acuity for reading, and that the perception of other (nonsymbolic) visual patterns is undisturbed-else one has sumply a loss of vision or a visual agnosia. In the second case of motor "aphasia," what is reported by Kennedy and Wolf is a loss of the capacity for drawing, with no disturbance of anything related to speech a rare and valuable observation, but not apparently relevant to the questron of aphasia. Again, Kennedy and Wolf have provided no detals of sensory and motor tests, and these detarls must be given if it is to be shown that such a thing as a pure alexia or agrapha can exist. Nesther, certaunly, has yet been shown to result from damage to any limited part of the speech area It is hardly likely that they will be, such conceptions arise from an older type of theory, no longer entertaned in psychological thought, and it is bughly probable that such questions (concerning what pecultar contribution each part of the cortex makes to behavior) will remain unanswered untl we can find out how to ask better questions. 


\section{The Growth and Decline of Intelligence}

There have been a great many rateresting and important observations showing a relation of complex functions to the parjetal and temporal lobes in man, but in all the reported cases, of vanous agnosias, dusturibañces of the body umage, and so on, there is presumptive endence of the presence of pathological dysfunction as well as of a smple loss of tissue, and we are not yet in a position to interpret such data as far as the localization of function is concerned. Von Monakow wrote that he dud not know of a single case in which a purely traumatic leston in the occipital lobes of an otherwnse sound brain had resulted in a persistent visual agnosia. Thus, inplying that agnosia is an effect of dysfunction and not of an uncomplicated loss of cells, is directly in line with the present discussion (though it should be noted that a traumatic lesion, accompanied by scar formation, may also produce chronic dysfunction) There is little that can be defintely concluded at present concerning localization of the functions disturbed in agnosia, and so on, untll the disturbance is found to be produced by a clean surgical lesion, unaccompansed by delta waves in the EEG.

Only one thing need be added although the frontal lobe is the favonte_place in which to localuze higher functions when one is speculating about these matters, it is still true that there is no proof that any single bigher function depends on this part of the brain ( $\mathrm{He} \overline{\mathrm{b}} \mathrm{b}, 1945 \mathrm{~b}$ ) At least as good a case might be made out for the parietal and temporal lobes as the seat of man's distinctive psychological characteristucs-if these in fact depend on one part of the brain more than another (see, for example, Kubitschek, 1928, Keschner, Bender, and Strauss, 1938)

Because of the enormous amount of space that has been devoted to the effort to show that the prefrontal lobe is the seat and habitation of some higher function or other (there are few mental processes that have not been ascribed to it), the basis of these negative conclusions must be made more detalled. Doing so will also illustrate other points dealt with in the preceding pages and make them more specric

- Personal communication from Dr X S Lashley My interpretation of this observation is based on the fact that a traumatic lesion is less Lkely to produce chronic dysfunction than a tumor or abscess. 


\section{Anatomical and Pathological Data}

The first and most effectuve piece of evidence is the bistory of a patient both of whose prefrontal lobes were removed by Dr. Wilder Penfield, with results that radically changed the possibilities of surgical therapy in the frontal area Before this tume, it was universally accepted that loss of both frontal poles must be followed by gross deterioration of personality and intellect. Dr. Penfield's patient completely reversed the prediction, by being psychotic before operation (when the damage was relatively small) and quite indistingushable from a normal person afterward (when the total destruction was increased in szzewith the simultaneous removal of a source of physiological disturbance).

The history is that of a boy of sxteen whose skull was fractured, with damage to both frontal lobes, and who in the following ten years developed severe epilepsy and became (much of the tume) an irresponsible and rather dangerous charge The condition varied, not only with the occurrence of attacks but also between attacks-apparently owng to subclunical seizures. the IQ, on the basis of an uncompleted testing on one day, was estmated to be below 70, next day a completed test gave an IQ of 84. After operation, the IQ was consistently close to 96 , no evdence of intellectual loss could be found in any one test or in the pattern of test scores as a whole, and the psychotic bebavior completely disappeared. Clincally and socially he was "normal" and was able to take full control of his own affarrs on the last examination he had been dong so for more than tive years. He enlusted in the army and served satisfactorily overseas for ten months untl he had another fit-at which time the medical officers caught up with him and he was discharged In his subsequent bebavior it is possible that there were deficiencies in planning for the distant future, but it is certain that he had more intrative and planned foresight than many persons with frontal lobes intact.

There must of course have been some loss of functron in this patient. Logically, it seems impossible to suppose that behavior can be unchanged after bilateral loss of any area of the brain. My conclusion is not that the frontal lobes-have no.function, but ether (1) that we have not yet guessed at the real nature of 


\section{The Growth and Decline of Intelligence}

this function-have not asked the proper questions in such investigations, or (2) that once the development of behavior has been completed the prefrontal lobes have no great further importance. Surely it is the first of these, 1, that is the answer, not 2, but there is nothing in the evidence at present that justifies the idea that the frontal lobes haye some special relation to hugher.functions in human behavior.

This general conclusion does not actually rest on the one case that I have described but can also be established independently. The endence that has been supposed to show the importance of the frontal lobes does not withstand scrutiny (it has been renewed in detall elsewhere-Hebb, 1945b). What the Penfield case did was, first, to demonstrate conclusively that an area in whuch there is dysfunction (the area of scarning that produced both eplepsy and deterioration of behavior) can be far more deteriorating than complete removal of the area, and, second, it rassed the questoon, How are we to account for the astonshing difference between this case and other, apparently similar, cases, in which, however, profound deterioration remained after surgical operation on the frontal lobes?

Eramination of the other case reports at once showed strong reason to believe that the deterioration they described was due to dysfunction, or diffuse destruction in the rest of the brain resulting from the earlier pressure of a tumor, or both. It thus appeared that the behavior changes were not due to the surgical injury, and therefore that such cases are not suitable for studying the localuzation of function The extent of the total lesion in each case was not known, and the loss of tissue was complicated by additional factors Dysfunction is to be expected also in frontal lobotomy (p. 272), and so cases of lobotomy also are not a source of interpretable data for the localization of function, especially sunce we do not know at all what the effect would be of a similar bilateral mowion in other regions of the brain.

Ths line of argument then obtained confirmation from the reports of bilateral prefrontal lobectomy by Mixter, Tillotson, and Wies (1941) and Heath and Pool (1948) Equally important, It now appears that monkey or chimpanzee may lose both frontal 


\section{The Growth and Decline of Intelligence}

this function-have not asked the proper questions in such investigations, or (2) that once the development of behavior has been completed the prefrontal lobes have no great further importance. Surely it is the first of these, 1, that is the answer, not 2, but there is nothing in the evidence at present that justifies the idea that the frontal lobes haye some special relation to hugher.functions in human behavior.

This general conclusion does not actually rest on the one case that I have described but can also be established independently. The endence that has been supposed to show the importance of the frontal lobes does not withstand scrutiny (it has been renewed in detall elsewhere-Hebb, 1945b). What the Penfield case did was, first, to demonstrate conclusively that an area in whuch there is dysfunction (the area of scarning that produced both eplepsy and deterioration of behavior) can be far more deteriorating than complete removal of the area, and, second, it rassed the questoon, How are we to account for the astonshing difference between this case and other, apparently similar, cases, in which, however, profound deterioration remained after surgical operation on the frontal lobes?

Eramination of the other case reports at once showed strong reason to believe that the deterioration they described was due to dysfunction, or diffuse destruction in the rest of the brain resulting from the earlier pressure of a tumor, or both. It thus appeared that the behavior changes were not due to the surgical injury, and therefore that such cases are not suitable for studying the localuzation of function The extent of the total lesion in each case was not known, and the loss of tissue was complicated by additional factors Dysfunction is to be expected also in frontal lobotomy (p. 272), and so cases of lobotomy also are not a source of interpretable data for the localization of function, especially sunce we do not know at all what the effect would be of a similar bilateral mowion in other regions of the brain.

Ths line of argument then obtained confirmation from the reports of bilateral prefrontal lobectomy by Mixter, Tillotson, and Wies (1941) and Heath and Pool (1948) Equally important, It now appears that monkey or chimpanzee may lose both frontal 
poles without evident umpairment, though earher work seemed to have establushed the contrary.

We have at present, then, no reason to throk that a clean surgical removal of both frontal association areas is necessarily followed by observable, clmically evident defects of behavior, nor bave we as yet any real understanding of the functions of this part of the brain.

\section{DIFFERENCES BETWEEN EARLY AND LATE BRAIN INJURY}

The study of brain operations in human patients has not so far provided the data that would allow one to be very definte about the way in which higher functions are related to specral parts of the cerebral cortex, but it has raised one problem that gives us a valuable lead concerning the nature of intelligence The problem has been referred to already-it anses from the discovery that an IQ of 160 or higher is possible after the removal of one prefrontal lobe (Hebb, 1939) or an IQ of 115 after hem1decortication (Rowe, 1937), and from the frequency with which above-average scores are reported after cortical mjury in any except the speech area. It appears possible, however, that similar damage to the infant bram has a much greater effect on the subsequent IQ. Thus possibjlity suggests a clue to the nature of adult intelligence and suggests a distinction between two quite different meanings of the term "intelligence"-a distunction that may help to resolve current theoretical disagreements.

The average IQ after bram operation, in all cases in which an IQ was reported up to the end of 1941, was 108-at least 8 points above the normal adult average, which is probably well below 100 according to the U. S. Army data of 1917 and Weisenburg, Roe, and McBnde's (1936) data. When brayn damage is traumatic or pathological, instead of surgical, there are also frequent indications that on certain kands of test (an which the Stanford Binet IQ is mainly based) the patient's score is not much lower than it would have been had the bram damage not occurred These data are given in more detail elsewhere (Hebb, 1942a).

- See T. Spaet and H F Harlow, J Exp Psychol, 1943, 32, 424-494, R. A Blum, Amer Psychol, 1948, 3, 237-238 


\section{The Growth and Decline of Intelligence}

But in practically all such cases the use of other tests shows that there is a definite intellectual loss. That is, some tests such as the Stanford Binet show hittle sign of a loss of intelligence, others show much greater loss There are then two kinds of test matenal, or two extremes in test content that shade into one another. The same picture appears when older adult subjects are examined: some (Binet type) tests show no deterioration due to advanemg age, others show a marked deterioration, and most tests fall somewhere between the two extremes (Jones and Conrad, 1933, Wechsler, 1939).

The Binet IQ is determuned by the use of a composite test, and it amounts to an average level of performance in a miscellany of tasks. It is manly verbal, however, and maunly mvolves a kiod of problem to which the answer is rather obvious, to the subject who can solve it at all. Tests of intelhgence may also consist of nonverbal tasks, and puzzle-like tasks-ones that require an effort and have to be worked out logically. Analysis of the test scores made after brain operation, when aphasia is not present, indicates that it is the first of these two kinds of task that is little affected by brain damage, the second that is much affected (Weisenburg and McBnde, 1935, Rylander, 1939; Hebb, 1942a) The IQ shows little effect of brain damage outside the speech areas because it is manly based on "Binet type" tasks. These consist partucularly of tests of vocabulary, information, digit repettion, digits backward, understandung of the meaning of fables or proverbs, and certain problems of a famular kind, dependent usually on common sense (in the form of a cultural sophistication).

Now, for normal persons, vocabulary and information tests are among the most valid tests of intelligence, and the Binettype test in general is what best predicts the subject's level of problem-solving outside the laboratory of clinc, in a wide range of situations. Thus is what presents us with our problem: how can they also be the ones that show the least effect of brain operation, and the degenerative changes of senescence?

But we must consider at once a further fact. that in cortain essential respects, "intelligence" does not decrease after the age of twenty or thirty, and the bratn-operated patient very frequently demonstrates an unimpatred level of functioning in his 
dally occupations. The patient with an IQ of 160 after prefrontal lobectomy (Hebb, 1939) graduated with honors in his arts course and went on to do well in medical school, the patients with half the cortex removed (Dandy, 1933, Rowe, 1937, Karnosh and Gardner, 1941) clearly did not act in any respect like the feebleminded, in therr daily affaurs. Certain intellectual capacities are well retained by the patient; in some respects, the prediction made by the Bmet-type test is valid. The puzzile is not why the Bunet suddenly loses its dragnostic value, after brain mjury. but why an important part of intelligence is little affected by the injury The fact that it is not, at once suggests that this part of intelligence is not directly correlated with the integnity of the brain, and, since the problems in which "intelligence" is unimparred are in general those with which the patient is familar, a further suggestion is that part of intelligence is a product of experience.

An sdea of this sort led to the examination of the effects of early brain injury (with the help of Dr Heinz Werner), the results, in short, indicated that any damage to the infant brain would affect later performance on vocabulary tests, information, and the like at least as much as performance on other tests (Hebb, 1942a) The situation apparently is this Unless every known case of large damage to the infant cortex is a case of speech-area damage (a most improbable situation), destruction of tussue outside the speech areas will prevent the development of verbal ablities, but the same destruction may not greatly affect these abulities once development has occurred

- The endence for thes interpretation is not perfect, but it is rather strong The one flaw appears to be the possibulity that the known cortical-type birth-injury population is made up predominantly or enturely of patients with speech-area lesions It is concervable that when palsy is not present only speech-area cases are detected-that all the "exogenous" cases of mental deficsency (Werner and Strauss, 1939) are discovered because of damage to this particular cortical area alone. But aganst thrs possibility is the fact that cases of palsy, presumably detected because of it, also have the low verbal test scores characteristic of other burth-ujury cases The twelve cases reported by Doll, Phelps, and Melcher (1932) showed marked rocabulary retarda- 


\section{2

tron. The low vocabulary score of the burth-mjured is not due to deficient opportunity to learn the meaning of words ( $\mathrm{Hebb}$, 1942a) - a conclusion supported by the almost identical retardations of vocabulary and digit-repetition scores reported by Doll, Phelps, and Melcher. Further, Doll (1933) reported that there is no correlation of speech defect and IQ in the burth-mjured

It appears, therefore, that an early injury may prevent the development of some intellectual capacities that an equally extensive injury, at matunty, would not have destroyed. To complete the picture, it should be sajd again that this relatonship does not hold-at least not to the same degree-for all intellectual capacities, and sensory and motor capacitues after damage to the infant brain tend to reach a higher level than that attaned after destruction of the same regions at maturity.

How are we to understand the first type of capacity in the adult, the one that is not greatly affected by brain damage at maturity? Psychologically, the matter might be put this way The actual modifications of behavior that occur in intellectual development are from one point of view qualitative. faced with a complex situation, the more intelligent subject sees it in a different way, and makes a different response-not more responses, or responses that take more effort. The chief intellectual effort, - in such behavior, is demanded on the first occasion on which the new way of seemg the situation occurs. Learning to solve a problem usually demands more effort than solving other problems of the same kmd on later occasions when one has discovered the principle involved. The same is often true of perception, whether one is solving a formal problem or not, once one has seen a certain relationship in a picture, or heard it in music, It may take no effort to find it again in simllar pictures or music This perception of relationships is essential in problem-solving, and one would steadily accumulate an increasing stock of such ways of seeing and attackung problem situations, as age advances, so, in a famljar field, the fifty-year-ald may be definutely more "intelligent" (as defined by the problems he can solve) than a twenty-year-old At the same tume, the problem that 'requires a radically new approach, new ways of percerving, is not lukely to be solved by the older man with his shrunken brain - Thie difference in the demands that are made by different pro- 


\section{Early and Late Brain Injury}

fessions, or different fields of investigation, would account for the fact that some fields are characteristically the domam of young men, others of the middle-aged. We cannot simply generalize, and say that intellectual capacity, objectively defined," decreases from the age of twenty-five onward. Some capacities do, others do not

$\checkmark$ Physiologically, the matter may be put as follows: some types of behavior that require a large amount of brain tissue for their first establshment can then persist when the amount of avalable tissue has been decreased This of course is consistent with the theory of cell-assemblies that has been presented in this monograph. It has been postulated that, with the enlargement of synaptic knobs, the number of fibers necessary for transmission at the synapse decreases ( $p$ 66) In the first establishment of an assembly, then, more fibers are necessary than for its later functioning.

Also, it has been sard that there is a possibility of "shortcurcuting" in the phase sequence ( $p$ 228) so that, after a phase sequence has been well established, some assemblies may no longer be necessary to it. If bram injury occurs in a limited region of the bram, it would presumably remove a small number of transmission paths from each of a very large number of assemblies. These assemblies might still function, though they would have smaller safety margins (p 197) and therefore would function less reliably. In other assemblies, the loss might be great enough to prevent any functioning; but a phase sequence in which some of these assemblies were originally essentral might later short-circuit them, and produce the same ultunate insight or understanding of the situation, though some of the steps of inference would have been omitted

Visual stimulation may have been involved in the formation of some concept, and yet not be necessary to its later arousal (p 128). Thus the congentally blund would be mcapable of unsights and understandung possessed by those who have become blund after havmg had vision In the same way, an area-18 lesion might prevent certain "visual" assemblies from functioning. These may have been onginally necessary, as a connectung link, in the establishment of connections between other assembles, constituting a particular conceptual activity, but once the 


\section{The Growth and Decline of Intelligence}

connections are established, the connecting link may not be needed any longer The same area-18 lesion in infancy thus might prevent a conceptual development but not destroy it at maturity.

\section{THE TWO MEANINGS OF "INTELLIGENCE"}

The clinical evidence has indicated, in effect, that there are two components in intelligence-test performance and in any intelligent behaviori 2 One is dimmished immeduately by damage to the brain, and amounts to a factor of heredity $y^{2}$ one is related more to experience, consistung of permanent changés in the organization of pathways in the cerebrum (in the present theory, these changes are the establishment first of assemblies of cells, and secondly of interfacilitation between assemblies). The hereditary factor is essentrally the capacity for elaborating perceptions and conceptual actrvities, the experientual factor is the degree to which such elaboration has occurred (and particularly, when we speak of matelligence, the conceptual elaboration that is not specific to one occupation or situation, but that enters into many human actuvities, concepts of number, of causal relations, of common human bebavior, and so on).

From this point of view it appears that the word "intelligence" has two valuable meanings One is $(A)$ an innate potentual, the capacity for development, a fully innate property that amounts to the possession of a good bram and a good neural metabolusm. The second is $(B)$ the functioning of a brain in which development has gone on, determining an average level of performènce of comprehenston by the partly grown or mature person Nerther, of course, is observed drrectly; but intelligence $B, a$ hypothetical level of development in brain function, is a much more direct inference from behavor than intelligence $A$, the original potentual (I emphasize that these are not two parallel knds of untelligence, coexustent, but two dufferent meanings of "intelligence.) It is true that estumating inteligence $B$ requires a summation of observations of behavior in many dufferent situatons, at different times; however, if we_assume that such an estmate is possible, what we actually know about an intelligence- 
test score is that it is primanly related to intelligence $B$ rather than intelligence $A$. The relationship to $A$ is less direct.

Most of the disagreement in recent years over the nature of "intelligence" concerns the relation of $A$, innate potental, to $B$, the estumated level of functioning at maturity! If $A$ determines $B$ fully, "intelligence" is a matter of heredity and maturation only, the IQ is not dependent on expenence. But if intelligence $A$ is only one of the conditions of intelligence $B$, not the sole determinant, what then? Intelligence $A$ is still hereditary, and it would not be true to say that "intelligence" (without qualification) is influenced by experience. only intelligence $B$ is so affected.

The dispute in the current literature has arisen, I belseve, partly because of tho double reference of the term "intelligence" and partly because it has not been realized that if the effects of early experlence are more or less generalized and permanent one can concede a major effect of experience on the IQ, and still leave the IQ ts constancy and validuty as an index of future performance.

An innate potental for development is not logically a guar- antee that the development will occur. There is in fact an overywelming body of evidence to show that experience is essentral to the development (the original potentiality is of course equally essentual). Binet humselfi assumed the necessity of adequate experrence in the subject to be examined by his method, the content of intelligence tests still makes this a necessary precond1ton; and all psychologists recognize that one cannot compare/s the innate intelligence of subjects from two different cultures. experience affects their IQ's to an unknown degree $V_{\text {Why then }}$ should we object to the Idea that enriching an inadequate env1ronment will rause the IQ, as Stoddard and Wellman (1940) and others have urged?

Some of the objection, certainly, may be occasioned by a faulty use of evidence and be due to the impression that the case for an environmental influence on the IQ has been overstated. But the roots of the objection, I believe, are to be found in certan 1deas about the nature of learning. It is apt to be assumed that learning is more or less specific to particular situations, that what is learned can always be forgotten, or that if something 


\section{The Growth and Decline of Intelligence}

can be learned in infancy it can also be learned at maturity Assuming the truth of such propositions, one might then think as follows:

$\sqrt{ }$ "If the IQ has any great dependence on learning it must Aluctuate easily, it might be raised from 100 to 150 in the adult, or ? might fall from 100 to 70 if a child is removed from school and other stimulating influences But the IQ does not in fact behave so-therefore learning does not contribute to it. It must be hereditary, because it is so stable"

However, there are definte limits to the "constancy" of the IQ, and most of the facts that seem to show that it is hereditarily determined would be accounted for if we assume that heredity ooly sets a limit to the IQ, and assume also that early learning tends to be permanent, that it has generalized transfer effects, and that it is not specific to particular situations That early learning has such properties has already been suggested, in the first part of Chapter 6. I wish now to report some preliminary experiments with the rat, which seem to show that some aspects of this animal's intelligence at maturity are a function of his early experience If this principle can be established by means of anumal expenument, we can then see how it would be applied to the koown facts of human intelligence and intellygence-test performance.

\section{EXPERIMENT INFANT EXPERIENCE AND ADULT PROBLEM-SOLVING}

When we test human intelligence, we always assume that the subject has had a certain body of experience common to all "normal" children in the community, the experience whose lack may invalidate comparison of the innate potential of subjects from different communties Apart enturely from controversy about the effect of special expenences on the IQ, we are all agreed, in this sense, on the effect of a certain kind of expenence (which is not, however, well defined)

One purpose of the experinental work briefly described here was to find out more about how the ordinary experience of infancy affects mature behavor. It was not certain that such effects could be found in rat behavior, even if they exist for man, 
but if one could find some trace of them in rat behavior their nature could be investigated much more easily in a species that reaches maturity in three months than in one that takes fifteen years Also, the clinical evidence reviewed in this chapter has indicated that the effect of early expenence is not found equally? in all forms of behavior Accordingly, these were exploratory experiments, with the main object of establishing the existence of some lastung effect of early experience on the later problemsolvng of the rat The first explorations gave wholly negative results, but they have the value of showing that some behavior is not affected by early experience, that 1s, they serve to delimit the effect.

In the first part of the study, one group of 7 animals were blinded in infancy, and a group of 7 hittermates were blinded at maturity. Both groups were handled frequently, and were allowed daly to run outside their cages in a large space in whoh there were a number of objects The question asked was this Would the group with vision during growth learn something about finding their way around that they would retain after being blinded at maturity? Would this affect their behavior permanently, in other situations than those in which their visual experience occurred?

At the age of five months-two months after the second group were blinded-the rats were traned to find food $\mathrm{m}$ one of four contamers in an open field. Two bundred trals were given, and the learning was not complete for most of the animals in this tume-so this presumably is to be classed as rote learming rather than unsightful A second period of training in a smular task also appeared to give rote learning, and in netther could any significant difference be found between the two groups, early- ard late-blinded

At thus pount, however, a chance observation suggested the possibility that an "intelligence test" for the rat (Hebb and Wllaams, 1946) might reveal differences between the groups Although the test had been meant for normal anmals, it might work with the blind as well.

The test showed a clear difference between the groups. By this tume the experment was being done with only 3 late-blended animals and 4 early-blinded, a small number for statustical sig- 


\section{The Growth and Decline of Intelligence}

nificance. However, there was no overlap of scores in the two groups-all 3 late-blinded were better than all 4 early-blunded, which is certanly significant."

Before repeating and confirming this result, it appeared profitable to explore further. The effect of vision during development, on the bebavior of rats after being blinded at maturity, was great, would other variations of experience also bave effects that could be detected by this-apparently sensitive-method of testung? The obvious experunent was to compare rats reared in ordunary small cages with others that had had the run of a wider envuronment. Two litters were taken home to be reared as pets, $\uparrow$ a first group of 3 (after 1 mortality at home) and a second group of 4. They were out of theur cages a good deal of the tme and running about the house. While this was being done, 25 cage-reared rats from the same colony were tested

When the pet group were tested, all 7 scored in the top third of the total distribution for cage-reared and pets. More important still, the pets improved ther relative standing in the last 10 days of testing, following 10 days of prelummary training, and 11 days of testing (a total of 21 tests was used) One explanation of the better scores of the pets is just that they were tamer, more used to handling, and less disturbed by testung. But, if this were so, the longer the cage-reared animals were worked with the closer they would come to the pet group, as the cageseared became tamer with prolonged handling. On the contrary, the pets improved more than the cage-reared. This means that the rtcher expertence of the pet group during development made

- The experiments described in this section are now berng sepeated and carred further by B Hymovitch and $H$ Lansdell, and will be reported in detail later The method has been considerably unproved since the first report of Hebb and Williams, it will be described in detal in a separate publication The essental fentures of the method are. (1) 10 to 14 days" prelununary traning gets the animal used to handling, to the test situation, and to daly changes in the route he must take to food, and teaches him where the food is to be found (its position is constant throughout), (2) the test itself is a serres of 20 to 24 separate problems, each rehtively easy for the seeng rat; he is given between 6 and 10 thals wh each, and his score is the total number of entries into error zones (see figure 17, p 137).

$f$ Grateful acknowledgments are due to the Misses Jane and Ellen Hebb, ages seven and five, for their enthusiastic assistance in the investigation 
them better able to profit by new expersences at maturity-one of the chatracteristics of the "intelligent" human being. Furthermore, a measure of motivation and tameness in the cage-reared was available, and the correlation of this with the test score was negligible $(0.13 \pm 019)$

$\checkmark$ These results show a permanent effect of early expertence on problem-solving at maturity. "permanent," because, in the first experiment with early- and late-blinded, visual experlence that ended at three months of age had marked effects at eight months of age (a long period in the life of a rat), and because, in the second experment, the pet group improved its standing cormpared to the others as expersence moreased Differences of early experience can produce dufferences in adult problemsolvng that further experience does not erase.

These preluninary results are already being confinmed by more elaborate experiments now goung on, and a principle is established that, first, is fully in accord with other evidence showng lasting and generalized effects of early experience (Chapter 6) and, secondly, ctanfifes the interpretation of existang data concerning human melligence test performance.

\section{THE NATURE AND NURTURE OF INTELLIGENCE}

Actually, some of the data on normal human intelligence (as well as the clinical data) allow only one interpretation, namely, that there is a major effect of experience on the IQ One diff-* culty in acceptung this interpretation is that it may seem to deny the validity of the intelligence-test method But, if the pronciple of a lastng and generalized effect of early learning is accepted, not only on the IQ but aiso on the everyday problem-solving of the adult, the validity of the intelligence-test method may be extended, not reduced. It is common to say that an intelligence test is not valud when given to a foresgner or a Negro. Not valud in what sense? as an estimate of mnate potentrality, of intellgessce $A$. It may be quite valid, on a purely empirical footung, for estimatung watelligence $B-$ the actual level of comprehension, learning, and problem-solving in this culture. Separating these two meanings of "intelligence" allows one to show where, the test is valud, as well as where it is invalud. 


\section{The Growth and Decline of Intelligence}

Look closer at this question of the relationship between test 'score and culture. It is agreed by psychologists that the IQ's of different peoples should not be compared, since intelligence tests are culture-loaded and not equally faur to subjects from different cultures. Now it is quite clear from the context of such discussion that the "unfairness" lies in estimatung a subject's hereditary endowment. Negroes living in the United States make lower average scores on intelligence tests than whites do, but we cannot conclude that the Negro has a poorer bram than the white. Why? Negro and white speak the same language, are taught in the same curriculum and sometimes in the same schools, work at the same plants, and on the whole intermingle very freely. Why then does the Negro's IQ not have the same meaning as a white man's? Because Negro and white do not have the opportunity to learn to speak the language with equal range and accuracy, are not usually taught in equally good schools, and do not have equally good jobs or equal exposure to cultural influences that usually require a faurly good income."

All this we can accept, but when we do so we must recognize that we have completely undermined the argument that dufferences of IQ among white, native Americans are determined by heredity.

In what respect does the argument, concerning the Negro's IQ and his innate endowment, differ for the poor white in the South and the white tenement dweller in the North? They do not have opportunity to acquire the same vocabulary, are often taught in poor schools, do not get good jobs or have good salanes The extent of this influence of experience is quite unknown, concervably, it is small, but one cannot argue that Negro and white IQ's cannot be compared, and at the same time that those of white and white can be, when the white subjects have different social backgrounds. The IQ can be trusted as an index - of intelligence $A$ only when the social backgrounds of the subjects compared are identical, and this adds up to the proposition that we cannot in any rigorous sense measure a subject's innate endowment, for no two socıal backgrounds are identical and we do not know what the important envronmental varables are in the development of intellectual functions Intelligence $A$ may 


\section{The Nature and Nurture of Intelligence 301}

sometimes be estimated, but it cannot be measured (Hebb and . Morton, 1944)

Intelligence in sense $B$ is a different matter. We know, beyond dispute, that the adolescent with generally low intelligencetest scores, whether Negro, poor white Amencan, or foreigner, is a poor prospect for college traming, or training as a mechanc, or Army officer, or dress designer. The inability to determine intelligence $A$ from a test score should not blind us to the fact that a foreigner's infellugence $B$ can be estumated, as far as its operation in this culture is concerned.

To be a bank manager, an aurplane pilot, a mathematician, a secretary, or a surgeon requires a certain common conceptual development that must occur in this or a closely related culture, and in childhood manly, and intelligence tests on the whole can provide a rather good index of the extent to which that development has occurred No other interpretation seems possible of the differences, $\vec{b}$ oth in educability and in intelligence-test scores, of first- and second-generation ummugrants Supposing that both have an adequate nutrition, father and son must have on the average the same intelligence $A$; the son very often has a much higher intelligence $B$.

$\checkmark$ The nature of the cultural environment that is necessary to this conceptual development cannot be described accurately. It does not necessarly consist of a formal schooling, and it may be present in spite of poverty In general, one might guess, it consists of an exposure to ideas, to books, and to intelligent conversation, the opportunity to acquure common technical knowledge and skills, and exposure to persons with social skalls, who are good at getting along with other persons. Besıdes being a guess, of course, such a statement is pretty vague and shows rather clearly how much we cannot say about the matter Also, we have no way of lnowing what cellng there is on this envronmental influence, at what age it is greatest, and so on. Such questions can be answered only by further research.

Our information at present is scanty, almost entirely naturalstic rather than experimental in orgin. Neff's (1938) review will show the interested reader how definite the evdence is. that environment has a major effect on the IO. Identical-twn data, for example, are commonly supposed to bave shown that 


\section{The Growth and Decline of Intelligence}

heredity is the only major determunant of "intelligence." In none - of the studied pars, however, was one twm brought up in an enturely favorable environment, one in an enturely unfavorable environment, usually they were brought up in about the same stratum of society, so that sumilar IQ's may be the result of simifar environment, a simular heredity, or both.

What we really want, as evidence on this point, is one twin brought up in a good home, with books, toys, kudergarten training, and plenty of exposure to intelligent adults, the other brought up by illiterate, poverty-stricken, ant1-social mountaineers with low IQ's then test their intelligences at maturity, and try makmg doctors, or politicians, of both twins. When this is done, and when it has been shown that the twin with a poor environment (for the first fifteen years of life) can with time become as good a duagnostician or committeeman as his genet1cally identical brother, it will then bo in order to say that the factual evidence shows that "intelligence" and the IQ are without any major effect from experience, not before.

If the Iowa studies (Stoddard and Wellman, 1940) are distrusted, the studies of Gordon, Asher, and Jordan, among others reviewed by Neff, all tell the same story. The constancy of the IQ is the main argument for its being determined by heredity, but the fact is that the IQ is not constant-1t is stable, and changes slowly, and more and more slowly as maturity is approached (Anderson, 1939), so that the IQ of the adult may be constant (though there are few data on long-term comparisons even here). But prediction of the adult $I Q$, in infancy, is more accurate on the basis of the parents' IQ's than on that of the infant humself, from an IQ at the age of five, prediction of the IQ at twelve is about 20 per cent better than chance prediction, very little better than can be done from knowing what kind of home the child is growing up in

- There are then two determmants of mtellectual growth a completely necessary innate potentral (intelligence A), and a completely necessary stmulating environment. It is not to the point to ask which is more important, hypothetically, wie might suppose that intelligence will rise to the limit set by beredity or environment, whichever is lower. Given a perfect environment, 
The Nature and Nurture of Intelligence 303

the mherited constitution will set the pace, given the heredity of a genius, the environment will do so

The essentials of this environmental infuence cannot be specified Though we know that wealth, prolonged schooling, or "intelligent" parents (that is, with intelligence B) are not essential, these things all may contribute. 'Sunce the guess has been made that the essential is exposure to intelligence $B$, it is presumably true that the child must exther have intelligent parents or intelligent acquaintances and teachers Schooling also is becoming more and more necessary to an understanding of adult problems in this society, and a certain amount of wealth, of freedom from economic pressure, may be quite necessary to full intellectual development. The fact is, however, that we know almost nothung specufic about the matter. The country may bo full of potential genuses, for all we know, and it should be a pressing concern for psychology to discover the condinons that will develop whatever potentralines a cbuld may have. 



\section{Bibliography}

Adams, D K 1829 Expermental studies of adaptave behavior in cats Comp Psychol Monog, 6, No 1

Adran, E D 1951 Potentral changes in the solated nervous system of Dytiscus margtnalss J Physiol, 72, 132-151

Adnan, E D 1934 Electrical actwity of the nervous system Arch. Neurol. Psychiat, 32, 1125-1138

Adran, E D, and Buytendyks, F J J 193l Potential changes in the isolated brain stem of the goldfish. I Physiol, 71, 121-135

Adrian, E D, and Matthews, B H C 1934 The interpretation of potential waves in the cortex I Phystol, 81, 440-471

Allon, C, and Broster, L R 1945 A further case of paranond psychosis successfully treated by adrenalectomy Brit Med J, No 4402, 696-698

Allport, G W 1946 Effect a secondary principle of learning Psychol Reo, 53, 835-347

Anderson, J. E 1939 The limitations of infant and preschool tests in theA measurement of intellugence I Psychol, 8, 351-379,

Arvanitaki, A 1942 Effects evoked in an axon by the activity of a contguous one I Neurophysiol, 5, 89-108

Bard, P 1984 On emotional expression after decortication with some remarks on certain theoretical vews Psychal. Rev., 41, 309-329

Bard, P 1942 Neural mechanisms in emotional and sexual behavior Psychosom. Med, 4, 171-172

Bartley, S H, and Bushop, G H 1933 Factors determining the form of the electrical response from the optic cortex of the rabbit Amer $J$. Physiol, 103, 173-184

Bartley, S H, and Chute, E 1947 Fatigue and impaiment in man New York McGraw-Hill

Beach, F A 1987 The neural basis of innate behavior I Effects of cortical lesions upon the maternal behavor pattern in the rat $J$ Comp Psychol., 24, 303-439

Beach, F A 1939 The neural basis of mnate behavior III Compartson of learning abulty and instinctuve behavior th the rat, $J$ Comp Psychol, 28, 295-282

Beach, F A. 1942 Analysis of factors involved in the armusal, mantenanco and manifestation of serual excitement in male anumals Psychosom Med, 4, 173-108

Beach, F A 1947a A review of physiological and psychological studies of sexual behavior in mammals Phystol Rev, 27, 240-307 
Beach, $f$ A $1047 b$ Evolutronary changes is the physwologral control of mating behavor in mammals. Psuchol Reo, 54, 297-315

Beach, F. A 1948 Hormones and behavior Now York Hoeber

Bellak, L, and Willson, $E$ 1947. On the etrology of dementra pracox - J Nerv Ment Dis, 105, 1-24

Bellows, R. T 1939 Tyme factors in water drinking in dogs Amer. J. Phystol., 125, 87-97

Burch, H G 1945 The rotation of provious expenence to insightful problem-solving I Comp Psychol, 38, 867-383

Bushop, G. H 1946 Nerve and synuptic conduction Ann Rev Phystol, $8,355-374$.

v Bonin, G, Garol, H W, and McCulloch, W S 1942 The functional organization of the accipital lobe In Kluver, H, Visual mechassoms. Biol Symp, 7, 165-192

Borting, E G 1816 Cutaneous sensation after nerve-diviswon. Quart J Exp Physiol, 10, 1-85

Bonng, E G. 1930 A new ambiguous figure. Amer J Psychol, 42, $444-445$

Bonng, E G 1933 The physucal dimensions of consctoumets New York Century.

Borng, E. G 1946 Mind and mechanism Amer I Psychol., 59, 173102.

$r_{i}$ Bousfeld, W A. 1935. Quantitative induces of the effects of fasting on eatung-behavior $J$ Genet. Psychol, 46, 476-479.

Bowman, K M 1985 Psychoses with pemucious anemia Amer J. Psychiat, 92, 871-396

Bowman, K. M 1946 Modern concept of the neuroses I Amer Med. Assoc, 132, 555-557

Bridgman, C S, and Smith, K U 1945 Bllateral neural integration in visual percepton after section of the corpus callosum. J. Comp Neurol, 83, 57-68

Bronk, D W 1939 Symaptuc mechanismus to aympathetic gangha $J$ Neurophysial, 2, 380-401

Brown, Wamer. 1932 Spatial integrations in a human maze Unto Collf Publ Psychol, 5, 123-134

Bruetsch, W. L 1947 Rheumatic brain disease late sequel of rheumatic fever. I Amer Med. Assoc, 134, 450-454.

Carlson, A J 1916 The control of hunger in health and disease. Chicago: Univ Chic Press

Carmichael, L, Hogan, H P, and Walter, A A 1932. An experimental study of the effect of language on the reproduction of visually percenved form $J$ Exp Psychol., 15, 78-86

Chandler, A R 1934 Beauty and human nature New York. AppletonCentury.

Clark, G, and Lashley, K S 1947 Visual dusturbances following frontal ablations in the monkey Anat Rec, 87, 326 


\section{Bibliography}

Cobb, S 1944 Personality as affected by lesnons of the brain In Hunt, $\mathrm{J} \mathrm{McV,} \mathrm{Personality} \mathrm{and} \mathrm{the} \mathrm{behawor} \mathrm{disorders} \mathrm{New} \mathrm{York} \mathrm{Bonald,}$ Vol I, $\overline{\mathrm{pp}} \mathbf{5 5 0 - 5 8 1}$

Cobb, S, Cohen, M E, and Badal, D W 1946 Capillaries of the nal fold in patrents with neurocirculatory asthenla (effort syndrome, anxiety neurosss) Arch Neurol Psychuat, 56, 643-650

Cohn, \& 1945 Electroencephalographic study of prefrontal lobotomy Arch. Neurol Psychtat, 53, 288-288

Cowles, J T, and Nissen, H W 1937 Reward-expectancy in delayed responses of chumpanzees I Comp Psychol, 24, 345-858

Dandy, W E 1939 Physioloric studies following extirpation of the neht cerebral hemsphere in man Bull. Johns Hopkins Hosp., 53, 31-51

Danel, I S., and Smith, K U 1947 Tho sea-approach behavior of the neonate loggerhead turtle I Comp Physol. Psuchol, 40, 413-420

Dashell, J. F, 1928 Are there any native emotnons? Psychol. Reo, 35, 319-327

Davison, C, and Demuth, E L. 1945 Disturbances in sleep mechanism a climico-pathologic study II Lesions at the corticodiencephalic level. Arch Neurol. Psychiat, 54, 241-255

Denker, P G 1946 Results of treatment of psychoneuroses by the general practitioner a follow-up study of 500 cases $N Y$ State $J$ Mad, 46, 2164-2166

Dennis, W 1934 Congental cataract and unlearned behavior J Genet. Psychol, 44, 340-350

Dennis, $W 1940$ Infant reaction to restruint an evaluation of Watson's theory Trans $N$ Y Acad Sat, Ser 2, 2, No 8, 202-218

Denny-Brown, D 1932 Theoretical deductions from the physiology of the cerebral cortex I Neurol. Psychopathol, 13, 52-67

Dewan, J G, and Owen, T 1945 Mental illness and the principles of medicine Canad Med Ass. J, 52, 349-357

Doll, E. A 1933 Psychological significance of cerebral bith lesions Amer I Psychol, 45, 444-452

Doll, E A, Phelps, W M, and Melcher, R T 1932 Mental defictency due to birth injuness New York. Macmillan.

$\checkmark$ Drew, G C 1838 The function of punishment in learning $J$ Genet Psychol, 52, 257-267

Dubner, II H, and Gerard, R W 1939 Factors controllhng brain potentsals in the cat $J$ Neurophysiol, 2, 142-152

Humlap, K. 1932 Habits thetr making and remakting New York Livenght

Edutors, Nutution Reviews. 1944. Self-selection of diets. Nutrition Rat, 2, 199-203

Egaña, E, Johnson, A E, Bloomfield, R, Brouha, L, et al. 1942 The effects of a diet deficient in the vitamin $B$ complex on sedentary men Amer I Phystol, 187, 731-741 
Erlanger, J 1989 The Imtation of impulses in arons. I Neurophusral. $2,870-37 \theta$

Ferraro, A, Aneta, S, and Englush, W H 1945 Cerebral changes in the course of permicious anemis and their relatioashap to poychic symp-" torns. J. Neuropathol. Exp Neurol., 4, 217-239

Fuelds, P E 1932 Studses in concept formation, I Comp. Prychol. Monog, 8, No 2

Forbes, A 1939 Problems of synapte function. I Neurophysill, 2, $465-472$

Freeman, G I 1994 Introduction to phystological psychology New York Ronald

Freeman, W., and Watts, I W IS42 Psuchosurgery intelligence, emothon and soctal behastor following prefrontal bbotomy for mental disorders Springfield Thomas

Freeman, W, and Watts, J W 1946 Psychosurgery. In Spiegel, E A, Progress tn netrologe and psychiatry an enneval reolew New York Grune and Stratton, pp 049-601

Fuchs, W 1920. Untersuchungen uber des Sehen der Hemianopuker und Herriamblyopiker II In Gelb, A, and Goldstein, K, Psychologischen Analysen himpathologischer Folle Leipzig Barth, pp 419-501

Fulton, I. F 1943. Phpsiology of the nerwous system 2nd Ed, New York Oxford Univ Press

Cante, W H 1988 Extension of a conflect based upon food to other physiological systems and its reciprocal relations with sexual functoons. Amer I Phystol, 128, 73-74

Gantt, W H 1944 Experimental basis for netrottc behavior origin and development of artifficially produced disturbances of behevior in dogs Psychosom Med. Monog, 3, Nas 3 and 4

Garrison, M 1947 The genetics of schurophrenta J. Abn Soc Psuchol, 42, 122-124

Gasser, H S 1837 The control of excitation in the nervous system Harvey Lect, pp 169-193

Gelleman, L W 1933 Form discrimunation in chimpanzes and twoyear-old children. I Form (trnangulanty) per se I Cenot. Psychol, 42, 3-27

Gtbbs, F A 1945 Electracal activity of the bram Ane Red. Phystol, $7,427-454$

Gibson, J J 1929 The reproduction of nsually perceived forms $J$ Exp Psychol, 12, 1-39

Gibson, J J 1941 A critical review of the concept of set in contemporary experimental psychology Psychol. Bull., 88, 781-817

Gibson, J J, and Crooks, L E 1988. A theoretical feld-analysis of automobrle driving Amer I Psychol, 51, 453-471

Gillesple, W. I 1944 The psychoneuroses J Ment Sa, 90, 287-308. Goodenough, F L 1931 Anger in young childrem. Minneapolis. Univ. Minnesota Press. 


\section{Bibliography}

Greene, R, Paterson, A. S, and Ple, G. C. S 1945 Hypertrichosis with mental changes the effect of adrenalectomy Brtt Med J, No 4402, 698-699

NGuthrie, E R 1946 Psychological facts and psychological theory Prychol. Butl., 43, 1-20

*Halstead, W C 1947 Brain and intellagence. Chicago Univ. Chic Press

Hanawalt, N G 1987 Memory trace for figures in recall and recogmbon. Arch Psychol, No 216, 1-89

Hìñns, H J 1944 Brucellosis a case report illustratung a psychosonatic problem Psychosam Med, 6, 834-335

Hauptman, $A$ 1948 Capillanes in the finger nall fald in patients with neurosss, opilepsy, and migrame Anch Neurol Psychiat, 58, 631-042

Head, H 1920. Studres in reurology Londan Frowde, Hodder and Stoughton

Heath, R G, and Pool, J L 1948 Bilateral fractional resection of frontal cortex for the treatment of psychoses J Nero Ment Dts, 107, $411-429$

llebb, D O 1937a The innate organization of visual activity I Perception of figures by rats reared in total daxkness 1 Genet Pauchoh, $51,101-120$

ILebb, D $01987 b$ The innate orgenization of visual actrutty- II. Transfer of response in the discrimunation of brightness and size by rats reared in total darkness J Comp. Psychal, 24, 277-299

Hebb, D O 1838. Studies of the organzzation of behavior I Behavar of the rat in field arientation J Comp. Psychol, 25, 393-352

Hebb, D O 1938 Studies of the organization of behinor II Changes in the field orientation of the rat after cortical destruction. $J$ Comp Psychol, 26, 427-444

Hebb, D O. 1039 Intellugence in man after large removals of cerebral tresue. seport of four left frontal lobe cases J. Gen Psychol, 21, 73-87

Hebb, D O 1942a. The effect of early and late brain unjury upon test scores, and the nature of nomal adult intelligence Proc Amer Phul Soc, 85, 275-292

Hebb, D O 1942b Verbal test material independent of speczal vocabnlary duffeulty J Educe Psychol, 38, 691-690

Hebb, D O 1945a. The forms and conditions of chumpanzee anger. Bull, Canad Psychol Ass, 5, 32-35

Hebb, D O 1945b Man's frontal lobes A cntical review Arch. Netrol. Psuchiat, 54, 10-24

Hebb, D O 1940a Emotaon in man and anmal an analysis of the mtutive processes of reconmition Peychol Reo, 53, 88-106

Hebb, D O 19166 On the nature of fear Psychol. Heo, 59, 259-278

Hebb, D O 1947 Spontaneous neurosis in chmpanzees theoretical relatians with clunical and experimental phenomena Prychosom Med, 9, 3-16 
Hebb, D O. and Foord, E. N. 1945 Erroxs of voual recogrition and the nature of the trace J Exp. Povchoh, 35, 335-348

Hebb, D D, and Morton, N. W. 1943 The McGll Adult Comprehenson Examination. "Verbal Situation" and "Piature Apomaly" Series. A I Eduo. Psychol, 84, 16-25

Hebb, D O, and Morton, N. W 1944 Note on the measurement of adult intelingence $f$ Gen Psychol, 30, 217-223

Hebb, D O, and Penfield, $W 1040$ Human behsvor after extensive bulateral removal trom the frontal lobes Arch Neurol Prychiat, 44, 421-488

Hobb, $D O$, and Riesen, A H 1943 The gentesis of rratronal fears Bull. Carad Psychol Ass, 9, 49-50

Hebb, D O, and Whllams, $\mathbf{K} 1941$ Expenmental control of cues determusug the rat's ormentation Bull Conad Poychol Ass, $7,28-83$

+Hebb, D O, and Willams, K 1946 A method of rating anmal intalligenos J Cen. Psychol., 34, 59-85

Hernck, C J 1929 The thinking machige Chicago. Univ Chic. Press. Hilgard, E R, and Marquis, D G. 1940. Conditioning and loarning New York Appleton-Century

Hoagland, H IS47 Enryme lonetics and the dynamict of behavior I Comp Physial Psychol, 40, 107-127.

Hoagland, H, Malamud, W, Kaufman, I C, and Pincus, G 1946 Changes in the electroencephalogram and $m$ the excretuon of 17 -ketosteroids eccompanying electro-shock therapy of agitated depression Psychosom Med, 8, 246-251

Hobles, C E 1941. Mentel discorder in one of a par of identacal twm Amer I Psychlat, 98, 447-450

Hobhouse, L T. 1015. Mind in ovolution. and Ed London Macmillan.

Howland, C I 1930 "Inhabition of renforcernent" and phenomena of expenciental extuction. Proc. Nat Acad Sch, Wosh, 22, 430-433 (Quoted by Illigard and Marquas, 1940, p 148)

Hovland, C I. 1937 The generalization of conditioned responses II The sensory generalization of conditioned responses with varying intenstiles of tone $J$ Cenct. Psychol, 5I, 279-291

Hull, $C$ L. 1834 The concept of the habit-fanily hiartichy and maze learring. Psychol. Reo, 41, 39-54, 184-152

Hull, C. I 1943 Princlples of behoolor an introduction to behavior theors New York. Appletan-Century.

Hull, C L 1945 The dxcorminatron of stmulus configurations and the hypothesks of afferent neural interaction Paychol Reo, 52, 133-142

Humphrey, $\mathrm{G} 1940$ The problem of the dureetion of thought Brit J Paychol, 30, 188-198

Hunt, J MoV, 1942 The effects of infant feedung-trustration upon adult hoarding in the albno rat. I Abm. Soc Pryohol, 36, 338-380 
Hunter, W. S. 1994 Learning IV Experimental studies of learnung In Murchison, C, Handbook of general experimental psychology Worcester, Mass Clark Univ Press, pp 497-570

Jackson, T A 1942 Use of the stuck as a tool by young chmpanzes J Comp Psychol, 34, 223-235.

Jacobsen, C F, Jecobsen, M M, and Yoshiokn, J. G 1932 Development of an infant chumpanzee durng her first year Comp Psychol Monog, 9, 1-94

James, W 1910 Principles of psychology New York. Holt

Jasper, H H 1937. Electrical signs of cortical activity Psychol. Buh, $34,411-481$

Jasper, H H 1941 Electroencephalography in Penfleld, $W$, and Enckson, T C, Epilepsy and cerebral localzzation Springfield Thomas, pp $380-454$

Jasper, H H, and Fortuyn, J D 1940 Experimental studies on the functional anatomy of peht mal epulepsy Publ Ass Res Nero. Ment Dis , 26, 272-298

Jefferson, G 1997 Removal of right or left frontal lobes in man Brit Med J, 2, 190-200

Jersild, A. T, and Holmes, F. B 1935 Children's fears New York. Teach Coll. Bux Publ.

Jollufe, N. 1942 The neuropsychatric manifestations of vitamn deficrencles. J Mt Sinat Hosp, 8, 658-667

Jones, H. E., and Conrad, H S 1933. The growth and decline of intelhgence a study of a homogeneous group between the ages of ten and sixty Genet Psychol, Monog, 13, No 3

Jones, H E, and Joues, M C 1928 A study of fear Chlldhood Educ, $5,136-143$

Jones, M C 1933 Emotional development. In Murchson, C, A handbook of chlld prychology, 2nd Ed Worcester, Mass - Clark Univ Psess, pp 271-302

Kappers, C U A, Huber, G C, and Crosby, E C 1936. The comparathos anatomy of the nervous system of vertebrates, Including man New York Macmullan, Vol. I

Karnosh, L J, and Gardner, W J 1941 An evaluation of the physical and mental capabitties followng removal of the nght cerebral hemisphere. Cleveland Clin Quart, 8, 94-108

Kennard, Mi A. 1939 Alterations in response to visual stmull followng lesions of frontal lobe in monkeys Arch Neurol Psychlat, 41, 11581165

Kennard, M A, and Ectors, L. 1988 Forced circling in monkeys following lestons of the frontal lobes $J$ Neurophysioh, 1, 45-54

Kennedy, F, and Wolf, A 1936 The relatonship of intellect to speech defect in aphasic patients $J$ Nerv Ment Dis, 84, 125-145, 293-311. Keschner, M, Bender, M, and Strauss, I 1938 Mental symptoms asso- 
clated with brain turnor a study of $\mathbf{5 3 0}$ venfied cases I Amer Med Ass , 110, 714-718

Kinder, E F 1027 A study of the nest-bulding activity of the albino rat J Exp Zool, 47, 117-161

Kinsey, A C, Pomeroy, W B, and Martn, C E 1948 Sexual behavior in the human male Phladelphin Saunders

Klebanoff, S G 1945 Psychological changes in organic brain lesions and ablations Psychol. Bull., 42, 585-623

Kle1tman, N 1939 Sleep and wakefulness Chrago Univ Chic Press Klmeberg, $O 1940$ Soclal psychology New York Holt

Koftka, K 1924 The growth of the mind New York Harcourt, Brace

Kofflk, K 1935 Princsples of Cestalt psychology New York Harcourt, Brace

Kohler, W 1925 The mentality of apes New York Harcourt, Brace.

Kohler, W 1929 Cestalt psychology New York Liveright

Kohler, W 1940 Dynamics in psychology New York Lvenght

Kohler, W, and Wallach, $H 1944$ Figural after-effects an investigation of visual processes Proc Amer Phil. Soc, 88, 269-357

Krechevsky, I 1932 "Hypotheses" versus "chance" in the pre-solution penod in sensory discrimination-learning Unto Callf Publ. Psychol., $6,27-44$

Krechevsky, I 1938 An expenmental investigation of the principle of proximity in the visual perception of the rat. J Exp Psychol, 22, 497-528

Kubitschek, P E 1928 The symptomatology of tumors of the frontal lobe based on a senies of twenty-two cases Arch Neurol. Psychrat, 20, $559-579$

Landis, C 1947 A modem dynamic psychology J Comp Physrol. Psychol, 40, 135-141

Landss, C, and Hunt, W A 1932 Adrenalin and emotion Psychol Reo, 39, 467-485

Lashley, K S $1929 a$ Bran mechantsms and intelligence a quantitative study of infuries to the brain Chicago Univ Chic Press

Lashley, K S $1929 b$ Nervous mechanisms in learning In Murchsson, $\mathrm{C}$, The foundations of experimental psychology Worcester Clark Univ Press, pp 524-503

Lashley, K S 1930 Basic neural mechanisms in behavior Psychol. Rev, 87, 1-24

Lashley, K S 1934 The mechanism of vision VII The projection of the retina upon the cerebral cortex of the rat J Comp Neurol, 60, 57-79

Lashley, K S 1937 Functional determinants of cerebral localzzation Arch Neurol. Psychiat, 38, 371-387 -

Lashley, K S 1938a Expenmental analysis of instinctive behavior Psuchol Rev, 45, 445-471 
Leshley, K S $1938 b$ The mechanism of vision XV Preliminary studies of the rat's capacity for detal vision. J Gen Psychol, 18, 123-193

Lashley, K. S 1988c The thalamus and emoton. Psychol. Rov, 45, 42-61

Lashley, K. S 1941. Patterns of cerebral integration indicated by the scotomes of migraine Arch Neurol Psychiat, 46, 331-339

Lashley, K. S $1942 a$ The problem of cerebral organzation in vision In Kluver, H, Visual mechanisms Biol. Sympos, 7, 301-322

Lashley, K. S $1942 b$ An examination of the "contunuty theory" as apphed to discrimination learning J Gen Psychol, 26, 241-265

Lashley, $K$ S 1944 Studies of cerebral function in learning XII Apparent absence of transcortical association in maze learning J Comp Neurol, 80, 257-281

Lashley, K S, and Clark, G 1946 The cytoarchitecture of the cerebral cortex of Ateles a critical examination of architectonic studies $J$ Comp Neurol, 85, 223-306

Lashley, K S, and Wade, M 1946 The Pavlovian theory of generalizaton Psychol Heo, 53, 72-87

Leeper, $R$ W 1935 A study of a neglected portion of the field of learmng-the development of sensory arganzation $J$ Genet Psychol., 46, 41-75

Leeper, R W 1948 A motivational theory of emotion to replace "Emoton as disorganized response" Psychol. Rev, 55, 5-21

Lehmann, J E 1937a. The effect of changes in the potassium-calcium balance on the acton of mammalian A nerve fibers Amer $J$ Physiol, $118,613-619$

Lehmann, J E $1937 b$ The effect of changes in $\mathrm{pH}$ on the action of mammalian A nerve fibers Amer $J$ Physiol, 118, 600-612

Levne, J 1945a. Studles in the interrelations of central nervous structures in binocular vision I The lack of bilateral transfer of visual discriminatuve habits acqured monocularly by the pigeon $J$ Genet Psychol, 67, 105-129

Levne, J $1945 b$ Studies in the interrelations of central nervous structures in binocular vision II The conditions under which interocular transfer of discrimmative habits takes place in the pigeon $J$ Cenet Psychol, 67, 131-142

Lewn, $\mathrm{K} 1938$ Will and needs In Ellis, W D, A source book of Gestalt psychology London Kegan Paul, Trench, Trubner, pp 283-299

Lbet, B, and Gerard, R W 1939 Control of the potental rhythm of the isolated frog brain $J$ Neurophysiol, 2, 153-169

Liddell, H S 1938 The experimental neurosis and the problem of mental disorder Amer $J$ Psychlat, 94, 1035-1041

Liddell, H S 1944 Animal behavior studies bearing on the problem of pam Psychosom Med, 6, 261-263

Lorente de Nó, $\mathbf{R} 1938$ a Synaptuc stimulation of motoneurons as a local process J. Neurophystol, 1, 195-206 
Lorente de No, R $1938 b$ Analysis of the activity of the chans of mternuncial neurons $J$ Neurophysiol, 1, 207-244

Lorente de N6, R 1939 Transmission of impulses through cranial motor nucler J Neurophysiol, 2, 402-464

Lorente de Nó, R. 1943 Cerebral cortex. architecture In Fulton, J F, Physiology of the nervous system. 2nd Ed New York Oxford Unv Press, pp 274-901

Lorenz, K 1935 Der Kumpan in der Urawelt des Vogels J Ornith., 89, $187-213,289-413$

Loucks, R B 1935 Expenmental delimitation of neural structures essentral for learnug The atternpt to condition striped muscle responses with faradization of the sigmoid gyrn $J$ Psychol, 1, 5-44

Loucks, R B 1938 Studies of neural structures essential for learning II The conditioning of salivary and strnped muscle responses to faradization of cortical sensory elements, and the action of sleep upon such mechanisms J Comp Psychol, 25, 315-332

Luckhardt, A B, and Carlson, A J 1915 Contrnbutions to the physiology of the stomach XVII On the chemical control of the gastric hunger mechanism Amer $J$ Physiol, 36, 37-46

McBride, A F, and Hebb, D O 1948 Behavor of the captive bottlenose dolphin, Tursiops truncatus J. Comp Physiol Psychol, 41, 111123

McCulloch, T L, and Haslerud, G M 1939 Affective responses of an infant chimpanzee reared in isolation from its knd $J$ Comp. Psychol., $28,437-445$

McCulloch, W S 1944 a Cortco-cortical connections In Bucy, P, The precentral motor cortex Urbana, III Univ Illmois Press, pp 213-242

McCulloch, W S $1944 b$ The functional organzration of the cerebral cortex Physiol. Reo, 24, $390-407$

\&McGeoch, J A 1942 The psychology of human leaming New York Longmans, Green

Marer, N R. F, and Schnerrla, T C. 1935 Princtples of antmal psy: chology New York McGraw-Hill

Marshall, W H, and Talbot, S A 1942 Recent evidence for neural mechanisms in vision leading to a general theory of sensory acuity In Kluver, H, Visual mechanusms Brol. Symp, 7, 117-164

Masserman, J H 1942 The hypothalamus in psychuatry Amer J Psychiat, 98, 633-637

Masserman, J H 1943 Behavior and neurosis An experimental psychoanalytic approach to psychobiologic principles Chicago Unv Chic Press

Matthews, R S 1938 Pellagra and ncotinc acid J Amer Med Ass, $111,1248-1153$.

Miller, G A 1947 The masking of speech Psychol. Bull., 44, 105-129 Miner, J B 1905 A case of vision acquired in adult life Psychol. Rev Monog Suppl, 6, No 5, 103-118 
Mixter, W J.; Tillotson, K J, and Wies, D 1941 Reports of partial frontal lobectomy and frontal lobotomy performed on three patients one chronc epileptic and two cases of chronic agitated depression Psychosom. Med., 3, 26-37

Morgan, C T 1943 Phystological prychology New York McGraw-Hill. Morison, R. S, and Dempsey, E W 1943 Mechanism of thalamocortical augmentation and repethtion Amer. J Physiol, 138, 297-308

Moss, F A (Ed) 1942 "Comparative psychology Rev Ed New York Prentice-Hall.

Mow̄rer, O H 1941. Motwation and learning in relation to the national emergency Psychol. Bull, 38, 421-431

Mowrer, O H, and Mowrer, W M 1938 Enuresis-a method for its study and treatment Amer J Orthopsychiat, 8, 436-459

Muenzanger, K F 1934 Mothvation in learnung I Electric shock for correct response in the visual discrimination habit J Comp Psychol, 17, 267-277

Murphy, J P, and Gellhom, E 1945 Further investigations on diencephalc-cortical relations and their significance for the problem of emothon $J$ Neurophystol, 8, 431-447.

Nafe, J P 1934 The pressure, pain, and temperature senses In Murchlson, C, Handbook of general expermental psychology Worcester, Mass Clark Univ Press, pp 1037-1087

Nauta, W J H 1946 Hypothalamic regulation of sleep in rats An expermental study J Neurophystol, 9, 285-916

Neet, C C 1933 Visual pattern discrimmation in the Macacus rhesus monkey $J$ Genet Prychol, 43, 163-196

* Neff, Walter S 1938 Socioeconomic status and intelligence a critical survey Psychol Bull., 35, 727-757

Nichols, I, and Hunt, J McV 1940 A case of partal bilateral frontal lohectomy. a psychopathological study Amer I Psychiat, 96, 1063 1083

Nissen, $H W$, Machover, $S$, and Kunder, E F 1935 A study of performance tests given to a group of native African negro children Brit J Psychol, 25, 308-355

Pavlov, I P 1927 Conditioned reflexes Oxford Humphrey Milford.

Pavlov, I P. 1928. Lectures on conditioned reflexes Now York Internathonal.

Pavlov, I P 1932 The reply of a physiologist to psychologists. Psychol Rev, 39, 91-126

Penfield, W, and Jasper, H 1946 Highest level seizures Res Publ Ass Nerv Ment Dis, 26, 252-271

Pennington, L A 1938 The function of the brain in auditory localizaHon IV Method of trauning and control experiments. $J$ Comp Psychol, 25, 195-211

Pilgrm, F J, and Patton, R A 1947 Patterns of self-selection of purl- 
fied dretary components by the rat J Comp Phystol Psuchol, 40, 343-348.

Pullsbury, W. B I913 "Fluctuations of attention" and the refractory penod J. Phll. Psychol Sct. Meth, 10, 181-185

Polyak, S. L. 1941. The retina Chicago. Univ Chic Press

Postman, L. 1947 The histary and present status of the law of effect. Psychol Bull, 44, 480-563

Pratt, C C 1939 The logtc of modem psychology New York Macmillan.

Prentuce, W C H 1946 Operationism and psychological theory a note Psychal Rev, 53, 247-249

Prosser, C L 1934 Action potentials in the nervous system of the crayfish I. Spontaneous impulses J Cell Comp Physiol, 4, 185-209

Ruchter, C P, Holt, L E, and Barelare, B 1938 Nutntional revurements for normal growth and reproduction in rats studied by the selfselection method Amer I Physiol, 122, 734-744

Ruddoch, G. 1941. Phantom limbs and body shepe. Bratn, 64, 197-222

Riesen, A. H 1947 The development of virual percepton in man and chimpanzee Solence, 108, 107-108

Riesen, A H, and Nissen, H. W 1942 Non-spatal delayed response by the matching technque J Comp Psychol, 34, 307-31s

Roethlsburger, F $\}$, and Dickson, W J 1939 Management and the woorker Cambridge. Harvard Univ Press

Romano, J, and Coon, G P 1942 Physiologe and psychologre studies in spontaneous hypoglycemin Psychosom. Med , 4, 288-300

Rowe, S N 1937. Mental changes following the removal of the nght cerebral hemisphere for brain tumor Amer I Psychiat, 04, 605-614

Rubin, E 1921 Visuell wahrgenommene Figuren Studien in psychologwaher Analyse. Teil I Berlin Gyldendalske Boghandel.

Rylander, G 1939. Personality changes after operattons on the frontal lobes a clinical study of 32 cases London Humphrey Mslford

Saphir, W 1945 Chromic hypochloremia sumulating psychoneurosss $J$ Amer. Med Ass, 129, 510-512

Schneirla, T C 1948 Psychology, comparative Encycl. Brtt

Scott, W W, Scott, C C, and Luckhardt, A B 1938 Observations on the blood sugar level before, during, and after hunger periods in humans Amer J Physiol, 123, 243-247

Senden, $M \vee 1932$ Raum- und Cestaltauffassung bet operierten Blindgeborenen vor und nach der Operation. Leipzag Barth.

Sherrington, C S. 1908. Integrative action of the naroous system. New York Serbbrer

Sherrington, C S 1925 Remarks on some aspects of reflex inhibition. Proc Roy Soc, 97B, 519-545

Shernngton, C. S 1941 Man on his nature New York: Mecmillan

Slanner, B F 1838 The behavior of organisms an experimental analysts New York Appleton-Century 


\section{Bibliography}

Smith, D E 1939 Cerebral lacalyzation in sornesthetic discrimination in the rat. J Comp Psychol, 28, 161-188

Surth, X U 1938 Visual discrumination in the cat III The relative effect of parred and unpaured stumuls in the discriminatuve behavior of the cat J Genet Poychoh, 48, 20.57

Smith, W K. 1945. The fumetional signsficance of the rostral cungular gyrus as revenled by its responses to electrical excitation $I$ Neurophystol, 8, 241-255

Spearman, C 1927. The abilities of man. New York Macmillan

Spence, K W. 1938 Gradual versus sudden solution of discrimination problems by chimpanzees I Comp Psuchol, 25, 213-224

Spence, K. W. 1940 Continuous versus non-continuous interpretations of discrimination learning Psuchol Reo, 47, 271-288

Sperry, R W. 1943 Visuomotor coordination in the newt (Triturus virdescens) after regeneration of the optec nerve $J$ Comp Neurol, 79, $33-55$

Sperry, R. W 1947 Effect of crossing nerves to antagonistlc hmb muscles in the monkey Arch Neurol. Psychiat, 58, 452-473

Spiegel, E. A, Millex, H R, and Oppenhermer, M J 1940 Forebrain and rage reactions I Neurophystol, 3, 539-548

Spies, T D, Aring, C D, Gelpenn, J, and Bean, W B. 1938 The mental symptoms of pellagra. Therr relief with nucotinse acid Amer I Med. Sct, 186, 461-475

Spragg, S D S 1940 Morphine addiction in chumpanzees Comp Peychol Monog, 15, No 7

Stoddard, C. D, and Wellman, B L. 1940 Enviromment and the IQ Yearb Nat Soc Stud Educ, 89 (I), 405-442

Stookey, B, Scarf, J, and Toitelbaum, M 1941. Frontal lobectomy in the treatment of brain tumors Ann Surg, 113, 161-169

Swank, A L, and Marchand, W E 1946 Combat neuroses development of combat exhaustion. Arch. Neurol Psychitat., 55, 236-247

Thomdike, E L 1931 Human learning New York Century

ThurstonerL L. 1935 The vectors of mind. Chicago Unv Chic Prese

Tunbergen, N 1942 An objectivistac study of the innate behavior of enimals Bibl. Biotheoret., Leaden, 1, $39-98$

Tunklepaugh, $O$ L. 1928 An experimental study of representative factors in monkeys I Comp Psychol., 8, 197-236

Titchener, E B. 1920 Notes from the psychologncal laboratory of Comell University Amer I Psychol, 31, 212-214

Tolman, E C 1932 Purposive bohadior in anumals and men New York Century.

Tryon, R C 1939 Studies in Indivdual duferences in maze learning VI Disproof of sensory components experimental effects of stimulus variation J Comp. Psuchol, 28, 361-415

Velentine, C W. 1930 The innate bases of fear J Genet Psychol, 97, $394-419$. 
Walker, A E, and Weaver, T A 1940 Ocular movements from the occipital lobe in the monkey J Neurophysiol., 3, 359-357

Watson, J B 1924 Behavrorism. New York Norton

Watts, J W, and Freeman, W 1946 Psychosurgery for the relief of intractable pan J Int Coll. Surg, 9, 679-683

* Wechsler, D 1939 The measurement of adult intelligence Baltumore Willams and Wilkng

Weddel, G, Sinclaur, D C, and Feindel, W H 1948 An anatomucal basis for alterations in quality of pain sensibility $J$ Neurophystol., 11, 99-109

Weisenburg, T., and McBnde, K E 1935 Aphasta a clinical and psychological study New York Commonwealth Fund

- Weisenburg, T, Roe, A, and McBride, K E 1936 Adult intellugence a psychological study of test performances New York Commonwealth Fund

Weiss, P 1941a. Autonomous versus reflexogenous activity of the central nervous system Proc. Amer Phil. Soc, 84, 53-64

Weisg, $P$ 1941b Nerve patterns The mechanics of nerve growth Growth (Thind Growth Sympostum), 5, 163-203

Werner, H, and Strauss, A 1939 Types of visuo-motor activity in their relation to low and high performance eges Proc Amer Ass Ment Defic, 44, 168-168

Wulson, G, and Rupp, C 1947 Present trends in the practice of neurology I Amer Med Ass, 133, 509-511

Wolf, E, and Zerrahn-Wolf, G 1937. Floker and the reactions of bees to flowers $J$ Gen. Physioh, 20, 511-518

Wolf, G A, and Wolf, H G 1946 Studies on the nature of certain symptoms associnted with candrovascular disorders Psychosom. Med, 8, 293-819

Wolf, H G 1943 Fmotions and gastre function Sclence, 98, 481-484 Wolf, H G, and Hardy, J D 1947 On the nature of pain Physiol Rev, 27, 167-199

Woodrow, $H 1927$ The effect of type of traming on transference J Educ Prychol, 18, 160-171.

Woodworth, R S 1821 Psychology New York Holt

Woodworth, R. S 1938 Experimental psychology New York Holt

Worts, H, Stem, M H, and Jollife, N 1942 Fiber dissociation in penpheral neuropathy Aich Int Med, 69, 222-237

Yerkes, $R$ M 1916 The mental life of monkeys and apes a study of ideational behavior. Behavior Monog, 3, No 1

Young, P T 1941 The expenmental analysus of appetute. Psychol. Bull., $38,129-164$

Young, P T 1944. Studies of food preference, appetute and dietary habit. I Ruoning activity and dietary habit of the rat in relation to food prefexence. J Comp Psychol, 87, 327-870. 


\section{Bibliography}

Zangwill, O L 1937 A study of the significance of attutude in recogurton Brtt J Psychol, 28, 12-17

Zener, $K 1937$ The signficance of behavior accompanying conditioned salivary secretion for theories of the conditioned response. Amer J Psychol, 50, 384-408.

Zollinger, A 1935 Removal of left cerebral hemisphere report of a case Arch Neurol Psychiat, 84, 1055-1064 



\section{Index}

Abstracbon, 104f

Adams, D K, 150

Adduction, 200-200

distinchion from habut, $202 \mathrm{f}$

mtermediate stage of, 203

learning $\mathrm{m}, 208 f$

relation to food habits, enotion, 205

Adrian, E D, 8f, 124, 184, 217, 274

"Afferent neural interaction," 15, 58, 79

Agnosite, $285 f$

Agraphı, 285

Alcohol as removing intibition, 209 if

Alexa, 285

Allen, C, 262

Allport, G W, 179

Analogy in scientific thought, 118 i

Anderson, ] E, 302

Anger (indtation, rage), see Rage

Angles in perception, 47, 82f

Animism, xilf, rvm, 4, 158, 173, 232, 234,286

Anxiety, causes of, 264

Aphasta, mteligence in, 161, $270 f$

locus of lesion m, $284 f$

Appette, 200

Area 17, motor effects from, 82, 86

Ariet, S, 262

Arng, C D, 262

Art appreciation, 233

Arvantald, A, 65

A/S ratio, 124f

and atturude, 144

and instinct, 167

and intelligence, 126, 165

Asher, E J, 302

Assembly, "Cell-assembly"
Assochatoon, varyng ense of, 77,127

Assocuation areas, sensory control of, I23/f, 146, 165, 167

Associstion cortex, stze of, ses A/S retio

Assumption, concerning EEG, 8

of sensory dominance, $3-6$

of structural trace, 12-16, 60, 62$63,228-231$

of synaptic ressstances, $60,62-66$, 228-231

Asthma, 260

relation to emotuon, neurosus, $287 f$

Attention, es factor in learning, 151153

defined empancally, 4

defined theoreticully, 87, 102

lack of unity in, 152

relatuon to expectancy, 87

Attutude, affectung monotonous work. $228 f$

problem of, 141-146, $207 \mathrm{f}$

"Autonomous central process," 4 ff

as attention, 4, 87, 102ff, 15I-153

as attitude, 141-149, 207f, 227

as expectancy, xvili, 87, 102

as insight ar hypothesis, 133f, 158165

as intention, 151-158

as interest, 6, 223

as set, 1024, 107f, 144

as spontaneous neural action, $\boldsymbol{T}$

control of, as motivation, $180 \mathrm{f}$

enduring selectivity of, 141-146

interaction with sensation, 146157

time period of, 78;, 142ff 
Backtracking in mazo leaming, 195

Badal, D W. 262

Bard, P, 189, 2835t

Barelare, B, 190, 198

Bartley, S H, 8, 208, 217

Beach, F, A., 5t, 269, 207, 215f, 201

Bean, W, B, 262

Bedwetting, as neturotic, 280

Beebe-Centet, J. G, 188, 204

Bellak, L, 263f

Bellows, R T, 280

"Belongingness," $176 \mathrm{ft}$

Berger, $H$, rvi, 8

Binet, A, 895

Binet-type intelligence test, If, $290 \mathrm{f}$

Burch, H G, 117, 165

Birth mjury and $1 Q, 2,275$, 201f

Bushop, G F, If, 8, 11, sif

Black, perceptron of, $105 t$

Blodgett, II C, 179

Blood content, effect on neural firIng. 148, 100 \&

us emotronal disturbance, 148, 295-200, 2585

Bloomfield, $R, 262$

Blum, R A., 289

Body image, disturbance of, 230

$\checkmark$ Boxin, C, 67, 70, 96, 213

Border (contonir) in perceptan, 82

Boredom and sleep, 217, 223

Borms, E G, 25, 22, 25, 74, 78, 185

Boussield, W An 292

Bowmen, X M, 260, 202

Brain inury, anatomical data $\mathrm{m}$, 282-289

effects of, mereased by local syschrony, 293

esturates of intelligence following, 27ef

hemidecorticution, 2, 285, 289, 291

infant os adut, 289-294

IQ following, 2f, 282-292

made of retentios of, $292 f f$

Brain szze and learning, 12sf and atturudes, 144

and sntellagence, 126, 165

Brodges, $\mathrm{C}$., 255
Bridgmam, C S, 96

Bnghtness, perception of, 41-44

Bmox, P, 289

Brodmann (cortical arens of), 6?, 213, 219

Brogden, W 1, 70

Bnonk, D. W, 8, 195

Broster, L R., 262

Broulha, L, 262

Brown, W, 135

Bruetsel, W L, 262

Bnms, M, 187

Buptendryk, F J. I, 9

Cejal, 5 \&, 230

Curlsom, A I, 192, 1976

Carmichael, 2., 2B, 111

Catatonia not an mhrbition, 212

"Cell-assembly," elteraghong paths un. 73

as unstable equilibrium, 100

bilateral development of, 89

disruption of, by blood content, 3965

by long activation, 225

by senspry relationships, 1485, 182, $189,250-253$

durstion of activity $\mathrm{m}, 74,143$

Eirst stage of growth, 69-78

fixed variable order of stumult, 89

"growth" of, 76f

statistical factors on, 74-78

in the selatwe perception, 100

interference between assemblies, $88^{\circ}$

in emotion, $251 f$

motor fuclitabon from, 87, 29 , 107

properties of, after brain tnjury, $298 \%$

in metabolic disorder, 197

role of synaptic knobs in, 72, 197

safety margin in functionung of, 197, 298

"Central excitatory mechanism," sf, 216 
"Central motive state," 8

in hounger, 191, 200

Chandler, A B, 233

Chimpanzee, intelfigence of, $100 \mathrm{f}$ mental illness in, 245-250 morphine addiction in, 203ff uonspatial delayed response by, 95 ono-tral learming by, 112, 114 perception in, 29,23

rage and fear $3 n, 241-245$ reased in darkness, 32, 108, 112 recogmibon by observer, 27, 116

Claute, B, 208

Cingular regzon and cunsciousaess, 219

Clark, G, 34t, 87, 88

Cobb, S, 2624

Coffia, T, 193

Cohen, M E, 262

-Cohn, R., 273

Coms and EEG, 122

Comic books, 280, 285

Campletron, as assoctative, 103 in heraranopia, 51

Concept, "core" ws "fringe" of, 181134

in post-operative intelligence, 2 not solely verbal, 128, 183 vanaluhty wo, 99,133

"Condenser" dischange of excitation, 216

Conditioned refiex, not elicited in sleep, 2281

theory of, 174-180

central formalation of, 180

in motrvation, 176-180

Conduction trom visual area, 51, 67

"Configuration theory," xvil, 39t endence from heursnopta, 51 summary of crituge of, 585 23. learning thecry, $15,59,114$, 158-101

Congentally blind, memory for color, 29, 32

memory for patterns, 28f, 32, 114 perceptzon of vertical and horzzontal, 805
Congenitally blind, percentual generabeation, 28f, 50 recogniban of faces, 114 slow learning not due to motivaton, $111 \mathrm{f}$ study of, 18 if

time to gain pattern viston, 325 Connections, neural, as the control of behanow, ivit

condiboned by time factors, 10F, $72,188 E$

Full' theory of, 176-180

in present theory, xax, 81, 72, $107 f, 180$

in prestrate area, 67-69

infant os odult, 107f

needs as promoting $177 \mathrm{f}$

Pavlov's theory of, 174

Contad H S, 200

Conscroumess, 144-146

cortucal orgenuzghon in, 1221

duffusity of finng $\mathrm{m}^{2}, 145,219$. 221

mode of dinft to intrinsic organination, $221 f$

emotion es land of, 147, 239E

not function of cortex alone, 218 not bocalized, 219

selation to anatomical structures, 219

relation to EEG, sensation, 9f, 123, 145F

Constitution, and 12, 295f, 300-903 and schizophrenia, 2085

wo experience, basis of false dichotomy, 168, 198, 245, 259, 261-264

conceptually distinguished, 209 169, 259

Contours to perceptron, 82

Control group, chosce of, 278-281 normal os clinucal, 278 use in ratung intelligence, 276-291 Convergence, on association, 1298 in growth of zssembly, 87f, 72f, 75

Coon, G. P, 262 
Cortex, not a functional unit, 218 organization of, in infancy, 121128

in sleep, 122, 217-222 in the wakng adult, 122f, 141146,219 ff, 234 intrinsic, 121-123 mode of change in, $221 f$

Cowles, J T, 159

Crooks, L E, 21

Crasby, E C, 68

Culbertson, J T, I

Dallembach, K M, 187

Danuel, R S , 168

Dashuell, J F, 147

Davison, C, 222

Deafferentation, functional, in sleep, 218

ws de-efferentation, 220 rationale of, $220 \mathrm{f}$

Decerebrate rigidity, $212 f$

Dempsey, E W, 218

Demuth, E L, 222

Denker, P G, $270 f$

Dennis, W., 241, $244 f$

Denny-Brown, D, 7

Deterioration, clinical identufication of, 275-281

of learnung ("fading"), $224 \mathrm{f}$

Determmism in psychology, xili See also Anirnism

Dewan, J G, 262

Dickson, W J, $220 f$

Diet, self-selection of, 190f, 198, $204 \mathrm{f}$

Duffusity, of conducton from visual corter, 51, 67

of finng, essential to consciousness, 145,221

essential to motor control, $220 \mathrm{f}$

Dockeray, F C., 240

Dog, intelligence of, 160

Doll, E A, 2916

Drawngs and perceptual content, 9lf

Drew, G C, 177, 189

Dubner, H. H, 8
Dunlap, K, 224

Duration of reverberatory activity, 73f, 143

Dusser de Barenne, J G, xvi

Dysfunction, comphcating bram injury, 282-288

Ectors, L , 84f

Edes, B, 187

EEG, see Electroencephalogram

Egaña, E , 262

Electroencephalogram, after electroshock, 273

after lobotomy, 272f

assumption concerning, 8

crude inder, 122, 283

essential in study of braun unjury, $283 f$

ongin of, 8

related to sensation, consclousness, 9, 122f, 211

Emotion, and anumsm, 236f

as disorganizer, 238-24I

defined, 148, 238

James-Lange theory of, 237 .

more than sham rage, $235 \mathrm{f}$

not conscious state, 147, 298

two classes of, $240 \mathrm{f}$

us "emotional disturbance," 238241

Watson's theory of, 241

Emotzonal disturbance, as guding learning, 138f, 150f

causes of, 87, 179, 242-244, 250 254, $267 \mathbb{I}$

classified, 251-254

due to conflict, 87, 148f, $251 \mathrm{f}$

due to metabolic change, etc, 148, 195-197, 205, 253f, 267世

due to sensory lack, 148, 179, 252 f due to strange envronment, 87 , $149,251 f$

in asthma, 267ff

mechanisms of, 250-258

mental depression, $248 f$

nonspectllc first stage m, 243f, $255 f$ 
Cortex, not a functional unit, 218 organization of, in infancy, 121128

in sleep, 122, 217-222 in the wakng adult, 122f, 141146,219 ff, 234 intrinsic, 121-123 mode of change in, $221 f$

Cowles, J T, 159

Crooks, L E, 21

Crasby, E C, 68

Culbertson, J T, I

Dallembach, K M, 187

Danuel, R S , 168

Dashuell, J F, 147

Davison, C, 222

Deafferentation, functional, in sleep, 218

ws de-efferentation, 220 rationale of, $220 \mathrm{f}$

Decerebrate rigidity, $212 f$

Dempsey, E W, 218

Demuth, E L, 222

Denker, P G, $270 f$

Dennis, W., 241, $244 f$

Denny-Brown, D, 7

Deterioration, clinical identufication of, 275-281

of learnung ("fading"), $224 \mathrm{f}$

Determmism in psychology, xili See also Anirnism

Dewan, J G, 262

Dickson, W J, $220 f$

Diet, self-selection of, 190f, 198, $204 \mathrm{f}$

Duffusity, of conducton from visual corter, 51, 67

of finng, essential to consciousness, 145,221

essential to motor control, $220 \mathrm{f}$

Dockeray, F C., 240

Dog, intelligence of, 160

Doll, E A, 2916

Drawngs and perceptual content, 9lf

Drew, G C, 177, 189

Dubner, H. H, 8
Dunlap, K, 224

Duration of reverberatory activity, 73f, 143

Dusser de Barenne, J G, xvi

Dysfunction, comphcating bram injury, 282-288

Ectors, L , 84f

Edes, B, 187

EEG, see Electroencephalogram

Egaña, E , 262

Electroencephalogram, after electroshock, 273

after lobotomy, 272f

assumption concerning, 8

crude inder, 122, 283

essential in study of braun unjury, $283 f$

ongin of, 8

related to sensation, consclousness, 9, 122f, 211

Emotion, and anumsm, 236f

as disorganizer, 238-24I

defined, 148, 238

James-Lange theory of, 237 .

more than sham rage, $235 \mathrm{f}$

not conscious state, 147, 298

two classes of, $240 \mathrm{f}$

us "emotional disturbance," 238241

Watson's theory of, 241

Emotzonal disturbance, as guding learning, 138f, 150f

causes of, 87, 179, 242-244, 250 254, $267 \mathbb{I}$

classified, 251-254

due to conflict, 87, 148f, $251 \mathrm{f}$

due to metabolic change, etc, 148, 195-197, 205, 253f, 267世

due to sensory lack, 148, 179, 252 f due to strange envronment, 87 , $149,251 f$

in asthma, 267ff

mechanisms of, 250-258

mental depression, $248 f$

nonspectllc first stage m, 243f, $255 f$ 
Emotional disturbance, possible vascular ongin of, 253, 268f related to adduction, food habits, 205

related to learning, $138 f, 147-151$, 242, 245, 254-258

vigor of response in, 258

with interruption of sleep, 222

Enghsh, W H , 262

Enuresis, 260

Equpotentality, and tachustoscopuc experiments, $40-50$

In the cell-assembly, 74

meaning of, $39 f$

sensory, 39,43

Equivalence of stumul, 14, 39, 836

Erlanger, J, 65

Excitability, affected by frustration, conflict, fear, 230, 233

affected by memory, 227-229, 233

hypothesis of, 227-231

Excitation, discharge of, 216 problem of, 208-211

Expectancy, as a form of ideation, xvil

factor in attutude, 14If

in emotional disturbance, 87, 149

in instunctuve behavior, $169 f$

in voluntary action, 144, 154-156

relation to set, attention, 5, 87, 102

"Experunental neuros1s," 249, 264

Eyeblunk, learned, $107 f$

Eyemovement, and contours, 82

and straight lines, 48, $82 f$

in motor equivalence, $155 \mathrm{f}$

in perception, $33-37,45,85-87$

Factor analysis, $\mathbf{x}$

"Fading" during learning process, $224 f$

Fatgue, mental, problem of, 208f, 211

Fear, causes of, classified, 251-254

in chuldren, $179,241,244$

in chintpanzees, 241-245

mild, factor in pleasure, $1330-233$

of darkness, 179
Fear, of mutilated or dead body, $243 f$ of snakes, 242f

of solutude, 252

of the strange, 242ff

pleasant ("thnill"), 182, 283

Feindel, W H , 183

Ferraro, A, 262

Fessard, A, 8

Fields, P E , 29

Figural after-effects, $54-58$

Figure-ground relation, 19-26, 89 flucturation of, 25f, $99 \mathrm{f}$ mechanism of, $99 f$

Finch, G, 114

Foord, $E$ N, 13

Forbes, A., 75

Forgays, D , 49

Forturyn, J D, 218

Fractonation, 76, 89, 97

Freeman, G L, 280

Freeman, W, 270, $272 f$

French, E G, 281

Freud, $S$, xt

Freudian theory of learning, 250

Frontal lobe, lobectomy os lobotomy, 272

removal, bilateral, in man, 287

tn monkey, chimpanzee, $288 f$

stimulation vs inhibition from, 212

supposed functions of, 272, 286289

Frustration, effect on learning, $138 f$ maintaining excitabilty, 230, 233 source of pleasure, 233

Fuchs, $W, 51$

Fulton, J F, 213

Gantt, W H, 264

Gardner, L P, $224 f$

Gardner, W J, 291

Garol, H W, 67, 70, 96, 213

Gamson, M, 263

Gasser, H S, 10

Gellerman, L W, 29, 39

Gellhorn, E, 218 
Idealısm, philosophic, xdv

Ideation in animals, xvill, $158 f$

"Identity," 26-91

Image, a forbudden notion, xvu assembly as sumplest instance of, 60

eyemovement a factor $2 n, 34$ llusion of, 36

Incidental learning, m lower species,

- $\quad 115 f$

memory, 151-158, 181

Infant brain, effects of injury to, 289-294

intrinsic organization in, 121-129

learning in, 111-116, 120-126, 228f, 298

Inhibition, 208-215

as loss of facilitation, $213 \mathrm{f}$

as excitation, from frontal lobe, 212

from suppressor areas, $213 f$

"Inhibition of reinforcement," 224

Innate, see Constitution

Insect, efficient first leaming in, 113

Insight (intelligence), 158-105

after brain injury, $298 f$

as function of experience, 110, 117, 105

definition of, 162f

defficulties of conception of, 162 164

in early us later learning, 115

mechanism of, 133-134, 164f, $298 \mathrm{f}$

not a separate process, $164 f$

related to motivation, 181

two critern of, 160

Instunctive behavior, 113, 165-170

Insulin shock, 269

Intelligence, after burth injury, 291f after hernidecortication, 2, 289, 291

as factor in learning, 158-165

clunical estumates of, 276 ff

development of, neurologically, 293

psychologically, 292
Intelligence, experience as factor un, $110,117,119 f, 291 f, 295-303$

human os animal, 161

not separate process from learning, $164 f$

of aphasics, $161,276 \mathrm{f}$

of chimpanzees, 114, $160 \mathrm{f}$

of dogs, 160

of tdentical twins, $301 f$

of pnmitive peoples, $119 f$

of rats, 161, 296-298

retention of, after braln injury, 276f, 279f, 286-294

in old age, $290 \mathrm{ff}$

neural mechanisms of, $293 f$

tests of, affected by sophistication, age, $279 f$

two types of, 290

two meanings of, 294-296

use of control group in ratng, 275-281

Intention, 151-158

Interactionism, philosophic, xdv, 230f

Interest, factor in sleep, 6, 223 varzabuluty of, 141, $207 \mathrm{f}$

Interference-pattern hypothesis, 15, 47,52

Intrinsic organization in cortex, $121 f$ behavioral manfestations of, 122, 221

mode of dnft to diffuse organizaton, $221 f$

IQ, after brain injury, 289-292

and heredity, 295f, 300-303

in identical twnes, $301 f$

limited constancy of, 296, 302

of foreigner or Negro, invald for intelligence $A, 299 f$

valud for intelligence $B, 295$, 299ff

of Negro vs poor white, 120,300 Isomorphusm, 52ff

Jackson, Hughlings, 283

Jackson, T A, 117

Jacobsen, C F, $24 \theta$

Jacobsen, M M, 246 
Gelperin, J, 262

Generalvzation, perceptual, 12-16, $27,88,104$

in congenitally blind, $28 f, 32,50$ with relative values, 41-44

Generalization gradient, 59, 79

Gerard, R W, 8

Gestalt theary, assumptions of, 2023 , 3f, 13, 17f, 23, 41, 55, 114 difficulty for, with figural aftereffects, 56-58

with hemianopia, 51

with memory, 15

of intelligence, 158-165

sensory emphasis in, 3f, 22ff

source of current ideas, 59

summary of cntique of, $58 \mathrm{f}$

Gibbs, F A, 8

Gibson, J J, 5f, 8, 21, 111

Gillespie, W H, 262

Goodenough, F L , 192

Gordon, H, 302

Gottschaldt, K, 23

Greene, R, 262

Growth, in cortcal organization, 76f, 122

in perception, $76 \mathrm{fI}$

synaptic, $60,62-66,228-231$

Gum-shy dog, 120

Guthrie, E R, 180

"Habit-famuly herarchy," 155

Halstead, W C, $279 f$

Hanawalt, N G, 13

Hardy, J D, 185

Harlow, H F, 114, 289

Harns, H J, 262

Haslerud, G M, 243

Heuptmann, A, 262

Head, H , 185, 285

Heath, R G , 288

Hemianop1a, 51f after frontal injury, $34 f$

Hemianopic completions, 51

Heredity, and IQ, 295f, 300-308 and schizophrenta, $263 f$
Heredity, vs environment, basis of false dichotomy in behavor, $166,198,245,259,261-264$ conceptually distinct factors, 166-169, 259

Herrick, C J, xiv

Hulgard, E R, 5f, 8, 61, 145, 175f, 179,224

Hinsey, L E , 271

Hoagland, H, 263, 273 -

Hobbs, G E, 264

Hobhouse, L T, 158f, 163

Hogan, H P, 26, 111

Holnes, F B , 252

Holt, L E , 190, 193

Householder, A S, xd

Hovland, C .I , 176, 224

Huber, G C, 68

Hull, C L , 5, 7, 15f, 38, 41, 56, 58, $79,154 f, 176 f f, 180,224$

Humphrey, G, dix, 134

Hunger, 190-200 and emotonal disturbance, $192 f$ arousal of, 199f, 202 as addiction, 200-206 chronce vs acute effects, 192 defined, 190 for spectic substances, 190f, $204 f$ innate factors in, $197 f$ learning effects $\mathrm{m}, 192-195$ mechanism of, 197-200 metabolic factors in, 195-197, 201-206

satuation of, 190, 194, 202, 205 vs appetite, 200

Hunger hormone, 197

Humt, J MeV, 109, 120, 272

Hunt, W A, 237, 281

Hunter, W S, 163

Hymovitch, B , 298

Hypersynchrony, see Local synchrony $\bullet$

Hypotheses in rats, 158, 161-163 difficulties of conception of, 162 spatal, 81-95 
James, W., 110, 237

James-Lange theory, 287

Jasper, II H., 8f, 211, 217, $219 f$

Jefferson, C, 282

Jersild, A T, 252

Johnion, R E, 262

Jolliffe, N, 188, 262

Jones, $H$ E , 241, 243, 290

Jones, M. C, 241, 243, 255

Jordan, A. M, 302

Kallinan, Fus 263

Rappers, C U. A, 68

Karnosh, L J, 291

Kaufman, I C, 273

Kennard, M. A, $34 f$

Kennedy, F, 285

Kinder, E F, 119, 168

Kinsey, A C, 216, 231

Klebanoff, S G, 284

Klestman, N, 6, 217f, 220f, 224

Klineberg, $O, 265$

Kofflar, X, 10, 12f, 28, 51, 111

Xohlex, W, xiv, 10, 12, 15, 23ff, 38f, 54f, 80, 117, 158f, 252

Krechevsky, I, 92, 111, 159, 161

Iandahl, $\mathbf{H} \mathbf{D}, \mathbf{x i}$

Landis, C. 237, 240, 263, 271

Lange, C, 237

Language as set of condiboned responses, 117

Lansdell, $\mathbf{H}, 208$

Lashley, K S , 10, 12ff, 19, 27, 29ff, B8ff, $67,80 f, 88 f, 86,90,93$, $99,115,117,145,154,159$, 166, 184f, 285, 286

Law of effect, 159, 176ff

Leaming, adult, and insight, 110, $115,158-165$

as function of early learning, 109-111, 119f, 228f

as remforcing existing connections, 127, 130

mechanism of, 126-139

phase sequence a pre-condition of. 128
Learning, adult, role of meaning in, $117,128,130-134$

constitutional factor ubiquitous, 198

contugunty not sufficlent for, 127

early, channelung effect of, 109 , 119

generalized transfer from, 109 111

possuble permanence of, 109 , 208-231, 296, 299

effect of, on emotion, 143f, 244f, 250f, 254-258

on hunger, 136f, 192-195, 197206

on intelligence (insight), 110, 117, 119f, 291f, 295-303

on sleep, 220

effect of emotion on, 138;, 150, 108

effect of sleep on, 221

"free" of specific cues, 129

incidental or unmotuvated, 151153,181

in lower species, $115 f$

inconstancy of ("fading"), 140, 2244

mistaken for instinct, 113

motor, 98, 101f, 107f, 134-139, 158-157

one-trial, in chimpanzee, 114

prinary stage of, 120-126

as establishung sensory control, 125

slowness in, and motivation, $111 f$

relation to A/S ratio, brain size, 124-126

relation to phylogenesis, 112-116 relation to set, attention, $108 f$

theory of, continusty, nomcontanuity, $114 f$

not based on adult data only, 110

os heredity, sec Heredity is envxomment 
"Learning theory," es attack on animism, 4 naive, 4,159

sophustaceted, 89, 159, 177

os configurationism, 15, 59, 114f, 158-101

Leeper, A W., 22, 111, 238ff, 254

Left and nght in psychology, 59

Left-raght distinction, paradigm of adult learning, $117 f$

Lehmann, J E, 8, 195

Letter-square, illusion of image, 38

Leucotomy, see Lobotomy

Levine, $J, 45$

Lewm, X, 228

Lubet, $B, 8$

Liddell, H S, 189, 264

Lloyd, D. P C., 213

Lobotomy, 271-274

hypothesis of effect of, $272 \mathrm{f}$

not surted to localization, 288

Incal synchrony, after lobotomy, electroshock, $272 f$

assumption concerning EEG, 8 complicating brain injury, 283f reinforced by learning, 121f, 220 in infancy, sleep, 122

in sleep, 217 \&

negating function, rationale of, 213f, 220 of

1 Localization of function in human brain, 282-289

Iimited knowledge of, 284

tumor cases not suted to, 282, 288

Lorente de N6, R, xVi, 10, 61, 63fi, $72 f, 75,142,210,218$

Lorens, K., 120, 106

Loucks, II B, 176, 221

Luckhardt, A. B., 190, 107, 201

McBrdde, A, F, 243

McBnde, K. E, 277, 281, 285, 2896

McCulloch, T L, $249 f$

McCulloch, W S, dd, 67, 70, 75, 96, 213f, 218

McGeoch, J A, 109f, 128, 131, 177

MacLeod, J. F, 193
Machover, S, 119

Malamud, W., 273

Mammillothalemic tract, 218

Marchand, W E, 262

Marquis, D G, 5f, 8, 81, 145, 175f, 179,224

Marshall, W H., 4dx, 43, 48, 53, 58, 82

Martn, C E., 216, 231

Meson, H L, 262

Massed v8. distributed practice, 224

Masserman, J H , 285, 264

Matthews, B H C, 8, 217

Matthews, R S, 262

Maze learning, 134-139

Meaning, 128, 130t, 183, 165

"Means-end-readiness," 145f, 153157

Melcher, R. T, $291 f$

Memory trace, assumptions concerning, $12-16,62-68,228-231$

dual mechonusm of, $61 \mathrm{f}$

related to generalization, 13ff

reverberatory, 12f, 61f

tume factor in production of, 62, $230 \mathrm{~F}$

Mensh, I N, 281

Mental hygiene, 261, 204-206

Mental illness, accompanying other conditions, 202

causes of, 261-271

Ignorance about, 249f, $260 f$, $205 F$

in chimpanzees, 245-250

nature of, 259-264

no separnte psychogenic type of, 259, 201f, 271

not specifically sexual, 249

recovery without special treatment, 267, 269-271

treatment by gengral practitioner, $270 f$

Metabolic factors in emotion, 195$197,202,205,253 f$

Migrame scotoma, 51

Muller, G A, 188

Miller, $H R, 28 B$ 
Mind as nenal activty, siv, To

See aiso Animism, Conbosousness

Mmer, $] \mathrm{B}, 114$

Mtchlon, $M, 40$

Mrrtet, W J, 272, 288, 288

Mnemonic trace, see Memory traco

v. Monakow, C., 286

Morgan, C T, svith $0,8,137,172$, $189,190 \%, 195,107,2004,218$

Monssor, R S, 218

Morton, N W, 277, 301

Motavafion, as bodily stimulingon, 1726

as theoretical corception, 188, 173-174, 181

channeled by pain, 182

defined as aspect of phase sequence, 181

In CR theory, 176-180

lons of, due to monotomy, 224-22,

following error, 224

neural os animustuc, 284

related to untight, 181

Motor control and diffuse firing, 2006

Motor effects from visual area, 82,89

Motor equivalence, 153-15T

and perception, $155 f$

and volution, 145f

is instunctuve behavior, 160-169

Motor facilitation from assembly. 86r, 98, 202

Motor loarning, 98, 101f, 107-109

equivaleuco $\mathrm{m}, 158-157$

In the maze, 184-189

Matros links in perception, S5-97, 89f

Mowre, 0 H, 102, 260

Mowter, W. M, 200

Mueazinges, K F, 177, 389

Mnon, N. L, 240

Murpiny, I $\mathrm{B}, 218$

Music and pleasure, 233

Nafe, J P., $283 f$

Nauta, W J H, 217, 219, 292

"Nexd" in learming theory, 176-180

Net, C, C, 29

Neff, $W \leqslant, 801 f$
Neolognsm, difficulty of recall, 118

Neuralgre, $185 t$

Neuroblotaxis, 68,65

Neurosis, eed Mental illnoss

"Neturosts, expermerctal," 249, 284

Nochols, I, 272

Nossen, H. W, 25, 118:, 159, 201, 248

Norisense syilables, learning, 128

"Nonsensory" figure, 21-28

Old ago, intelligenco in, \&, 290, 293f memory in, 2228f

sleep in, 220

Operabonalusan, 189

Oppenhermer, $\mathrm{M}_{4} \mathrm{~J}, 266$

Opposites in chrldren's learming, 117 t

"Optonal transmession," 10, 72

"Oscullation princeple," 7

Owen, T, 298

Pam, 181-180

as guidung leasning, 150

as strengthening response, 177 . $180 f$

classical thearies of, 183

defintion of, 189

is glans pents, 186

increased by hypofonction, 184t, $188 \mathrm{~F}$

irhibition of, 188, 272

pleasant, 182

relatwon to $C$ fibers, 188

reason for, 188 t

velation to expotion, 148, 182

reluef by lobotongy, hypothesse of 273

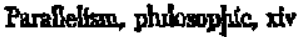

Paterson, A S, 869

Pattern of stimulation os locus, Ig I6, $39-41,44-50$

Pattox $\mathrm{A} A, 204$

Pavlov, I. P, xil, 5, 38, 11, 80, 174f, $180,189,212,284,284$

Pechstein, I, A, 115

Pentield, W, 219, 272, 2982, 2874

Penungton, L A., 88 
Perception, as action in specific cells, $25,17,42,44,48$

25 function of experience 22-24, 28f, 31-25, 111, 118E

duration of content of, 25, 74

equipoteatiahty in, 39-41, 44-50, 74

growth in, 77

innate organbation in, 236, 86f, $42 f$

integrabou of, pror to association, 77

unvaling conceptual activity, olf of bleck, $105 t$

of brghtness, 41-44

by raits reared in darkness, 42

hypothesis of, 485

of nearby us remole objects, 9195

of pattern, anstomical features in, 32-5\%, $57 \%$

and stomprplusm, 52-54, 57f

as summntive process, 18, 32, 34, 36 f

chenges with prolonged scare, 225

contours ra, 82

disbortions in cortex, 52-54, 57f

eyemovement in, 34, 36f, BQ102

figural afber-effects, $54-58$

"udenbty," 26-31

sdentrty function of experiEsce, 31ff

Blosery sumplicity of, $8 A$

integration of parts, 95-96, 103

Jocus of pattern, 12-15, 59.41, 44-50

"nomsensary figure," 21-20

part os whole in, 24f, 89t

"primitive unty," 19-21

recognistor of words, $40 \mathrm{f}$

right os left visual field, 50

whth varied angle of regard, 9 tF

with varled dextance, DDF

of pltch, size, 41f, 44, 106

phylogeneke differences $m, 29-91$
Perception, recurrent of cyclical, 25, 998

telatave, 41-44, 1055

Perceptual elements, 834

1n association of idess, 130

Perceptual generalzation, see Generalization

Phantom limb, pein in, 185

"Phase cycle," duratron of, 148

in perceptuon, 98f, 180f

"Phase sequetiog," 88-100

coudituons of persistence of, 142f, 202, 227, 220t, 232

confificts of, in emotion, 148-I51, 251-253

dommance of partly cuganized. 228, 232

flexibllity of, 87, 149

in mokvation, 181, 191

is simple perceptions, 996

relation to whong center, $218 \mathrm{f}$

self-destructura or perpetuation, 228

secosary dusturbanse of, 148-151, $251-253$

in pain, 150, 181-190

sensory puidsence of, 159-157, 182

sensony lacik, effect of, 148, 252f

short-crountang in, 228, 295

Pheips, W M, 20if

Fhylogenetic differences, in A/S ratio,

- brain size, 124-126, 167

In attitude, 141

in insight, 125f, 160t, $104 f$

in learing, 112-110

in perception, $20-31$

Pbysiologizing in psychology, xv

Physiology, relation of, in psycholOgy, XV

Psgenn, interoculat transfer wh, 45

Pise, G C. S, 262

Exlgrim, I 3, 204

Pilsbury, W B, 25, 74

Pincus, G, 273

Pitts, $W, \mathbf{x}$

Pleasent fear ("thrill"), 182, 293

Pleasant parn, $182^{\circ}$ 
Pleasure, 227-294

in art, music, sports, 293

relation to memory, 227-281

theory of, substitute for animism, 234

Polyak, S L, 52f

Pomeroy, W B , 216, 231

Pool, J L , 288

Position habit, 92, 161, 168

Positivism, xdv, 162

Postman, L , 177

Pratt, C C, xiv

Prentice, W C H, 162

"Primitive unity," 10-21

Probabulity of association, 127

in perceptual integration, 77

Prosser, C L, 8

Psychoanalysis, xil, 247, 261, 269-271

Psychosis, see Mental illness

Psychosomntics, 266-269

Psychotherapy, 261, 266-271

Purpose, 144-146

Rage (anger, imtation), causes of, $148,252,257 f$

in children, 192, 241

in chimpanzees, 241-245

not dependent on physical restraint, 242

not localized in hypothalamus, $235 f$

related to hunger, 192

sham, 235f

Ramsdell, D, 252 f

Rashevsky, $\mathbf{N}, \boldsymbol{x}$

Rat, hunger in, 193-195

intelligence of, 137, 161, 296-299

"Intelligence test" for, 135, 137, $297 f$

perception in, different from man's, 80f, 95

of durection, place, 02ff

reared in darkness, bnghtness discrumunation in, 42

figure-ground perceptlon in, $19 f$ slow pattern learning in, 81, 113

Rat, visual concepts after blinding, 129, $297 \mathrm{f}$

"Reaction to deficit," 166

"Reactive inhibition," 224

Reading speed, 49

Recognition of faces, 27, 114, 116, $127 f$

Recruitment in growth of assembly, 76, 97

in effects of lobotomy, 273

in sleep, 221

strengthening matrinsic organization, $121 f$

Relative perceptions, 41-44, $105 f$

Reverberation, bnef in smple cricunts, 73, $142 \mathrm{f}$

Ruchter, C P, 190, 193

Riddoch, G, 185

Riesen, A H , 18, 32, 78, 95, 108, $112,117,204,244,255$

Roe, A, 281, 289

Roethlusberger, F J, $226 f$

Romano, J, 262

Rowe, $S$ N , 2, 289, 291

Rubin, E, 19

Rupp, C , 260

Rylander, G, 279f, 290

"Salted-nut phenomenon," 199f, 202

Santa Claus vs mental hygrene, 200

Saphir, W, 102, 205, 262

Satiation, of hunger, 190, I94f, 202. 205

without sufficient food, 194, 205

of sex motivation, as neural discharge, 216

"Satuetion" of visual cortex, 54-58

Scarf, J, 282

Schema of perception, 69-74, 84$91,95-100$

"Scherma wh correction," 46, 111

Schneurla, T C, 168

Scientific method, xiv

Scientic thought, xiv, $118 \mathrm{f}$

Scott, C C, 190, 201

Seott, W W, 190, 201

Self-selection of dret, 190f, 193, 204f 
Senden, M v, 18f, 28f, 31f, 50, 78, 80f, 111f, 117

Senescence, intelligence in, 2, 290, 2926

mermory in, $228 f$

sleep $\mathrm{m}, \mathbf{2 2 0}$

Senson-motor connections, cutenon of, 108

linear theory of, xvil, 4, IOf, $40 \mathrm{f}$

modification of ides of, 180

sophustucated theory of, 59, 158, 177

of relative perceptions, 15f, $41 f$

Senson-sensory assocjation, 70

Sensory activity, cumulative effect of, 7, 128f, 128

in motivation, 172f, 177-179, 181

In sex behavor, $215 f$

in sleep, 217f, 220, 223

in voluntary acts, conscrousness, 9f, 123, 145f, 153-157

lack of, determining response, 166, 178, $252 f$

Sensory-central interaction, modes of, $87,146-157,232 \mathrm{f}, 250-253$

Sensory control, of cortex, 9f, 122127, 145f, $232 f$

and $A / S$ ratio, 125

as steering phase sequence, 87, 138f, 150f, I53-157, 181

establushed in prumary learning, 125

of motor activity, 107, 154-157, $167,220 \mathrm{f}$

variations in directness of, 107

Sensory dynamics in Cestalt theory, 3f, 10-25, 51, 54-58

Set, $4 f, 102 f f$

in voluntary act, 107F, 144

Sex behavior, as problem of motrvaton, 207

effect of novelty on, 231 . time course in, $215 f$

Sherrington, C S, 79, 182, 189, 212

Shock treatment, electrical, 271-274 insulin, 269
Short clrcuting in phase sequence, 298

and to retention of intalligence, 293

Shyness, 244

Similanty, 26f, 104

association by, 132

Sinclaur, D C, 189

Sze, perception of, 41f, 100

Sldnner, B F, xiv, 146

Sleep, 216-224

as causing relaration, 221

as extreme loss of motivation, 223

as intrinsic organization, $122 \mathrm{f}$

effect of, on learning, 221

effect of learming on, 220

unduced by cortical lesions, 292

interest a factor in, 217

mode of drift to waking state, $221 \mathrm{f}$

not a lack of organization, $219 f$

not a lowered cortical activity, 211

not only sensonly determuned, $21 B$

relation of, to sensation, of, 122f, $146,218,220,223$

smilarity to infancy and pathological conditions, 122

Sleep center, 217, 213

Smith, B F, 262

Smith, D E, 62

Smith, K U, 29, 96, 168

Smith, W K, 219

Sophustrated learning theory, 59, 159,177

Spact, T, 289

Spatial hypothesis, in rats, 91-95, 181, 168

Spearman, C, $\mathbf{x l}$

Speech area, localuzatuon of, $284 f$

Spence, K W, 41, 159, 163

Sperry, R W, 63, 157

Spiegel, E A, 238

Spies, T D, 262

Spontaneous neural activity, $8 f$

as problem in learning, 121-126

as regulated by sensation, 9

relation to motivation, $171 f$

Spragg, S D S, 203f, 248 
Stein, M. H, 188

Stumulation, physolosticals as dasrapting nomal function, 218i, 219

Stimulus, in motrotion theory, 172 pattem of, 12. lecus, 12-15, 39 $41,44-50$

Stimulus-response theory, see "Learning thenry"

Stoddard, G D, 295, 302

Stone, L. J, 187

Stookey, B, 282

Straight line, and eyemovement, 48, 821

learning to percenve, 81

physiological propertues of, $69 \mathrm{f}$

Stranss, A, 291

Supercosdinate perceptuons, 95-98

Suppressor areas, 2136

Swark, A L., 262

Symapte knots, and probability of transmisnon, 68, 76, 197, 293

as affecting newral control, 72 assunptions concernmg, 60-68, 228-231

formation of, 65f, 288-231

in one-trial leaming, 190

offsettung brain demage, 74, 2939

offsetting netabolic disorder, 197

permaneace of, 298-231

o*. thrme factot, 10\%, 72, 1986

Synchrony of fring, see Locel syeohrongy

Tachistoscople visson, and equipotencality, 48-50

essentials of, 47

number of hahit necenary for, 48

Talbot, S A, xn, 43, 48, 53, 58, 82

Tetelbewn, M , 282

Thalarms and consclousuress, 219

Thurst, Hanger

Thondike, E L, 264, 150, 1765, 180

Thought, ss ker problem, art-2viil restrocturing of, 134

See also Cousciousness; Phase quenco
Therstose, $\mathrm{L} \mathbf{L}, \mathbf{D}$

Tillotson, B 3, 272, 282, 288

Timberigen, N, 109, 113, 120, 100

Tinklegaugh, O L, 159, 258

Titchene, E $B_{1}, 41,187$

Tolman, E. C, 59, 148, 154, 159

Train of thought as phase sequence, Iix, $300,130-134$

Transfex of traming, 14 infant-adult, $109 f$ interocular, in pigeons, 45

Trensmustion, cortucel, and $A / S$ retio, 124f

as problem of thought, avt

Ttyon, R. C. 117, 129

"Vactumn ectrity," 160

Velentrne, C W, 288, 241

Varisbity, in motor response, 153357

of baraing, 140, 2245

Visual concepts in the blund, 129, 283

Vitalison, xivi

Seb also Animsm

Volition, 344fF

motor control in, 153-157

Wade, M., 27

"Wakefulnes of chouse" 218

Wolding center, 217, 219

Waking organizatuon, 122, 141-146, 2194, 234

mode of deft to satrensic organinatuon, 221f

Walker, A E, 88, 88

Wellach, H, 29, 64f

Waltet, A A, 2A, 111

Watson, I B, 40f, 2A1

Watts, I Wo, 270, $272 f$

Weaver, $T A, 82,80$

Wechsier, D, 990

Weddel, G, 188

Welsenburg, $T$, 877, 281, 285, 299

Weiss, $\mathcal{2}, 8,63,124,184$

Wellman, B. L., 295,302

Werner, H, 291 


\section{Index}

Wholes, untegration of, 95-98, 103

Wies, D., 272, 282, 288 Wilhams, K, 93, 135, 197, 188, 297 .

Willams, R D, 262

Willson, E, 2635

Wison, G, 260

Wolf, A, 285

WoIf, $E, 168$

WoII, G A, $200 \mathrm{E}$

Wolf, H. G, 185, $206 \mathrm{~F}$

Woodrow, $\mathrm{H}, 110$

Woodworth, R. S, 36, 46, 111, 131f,

$135,180,233,240$
Yerkes, $\mathrm{R}$ Mn $90,158 f$

Yerkes Laboratones of Primate B2ology, 114, 2425, 260

Yoshinka, J Co, 246

Young, $P$ T, 190, 193, 204, 240

Zangwill, O. Z, 20, 111

Zener, $\mathrm{K}_{\text {. }} 175$

Zener, Km 17 , G, 168
Zerrahn-Wolf,
Zollinger, R, 885 


\section{CENTRAL-INGTITUTE OF EDUCATION}

LIBRARY;

of $50.150 .1943 / 414511$

Ac. so. $46 \mathrm{~K} / \mathrm{G}$

Thas book abould be retaraed on or before the date leat atsumped below An overdue charge of one anna will be made for exolh dey the book it kept overbime Text books if overkept are lisble to a fine of As -/8/. pẹr day.

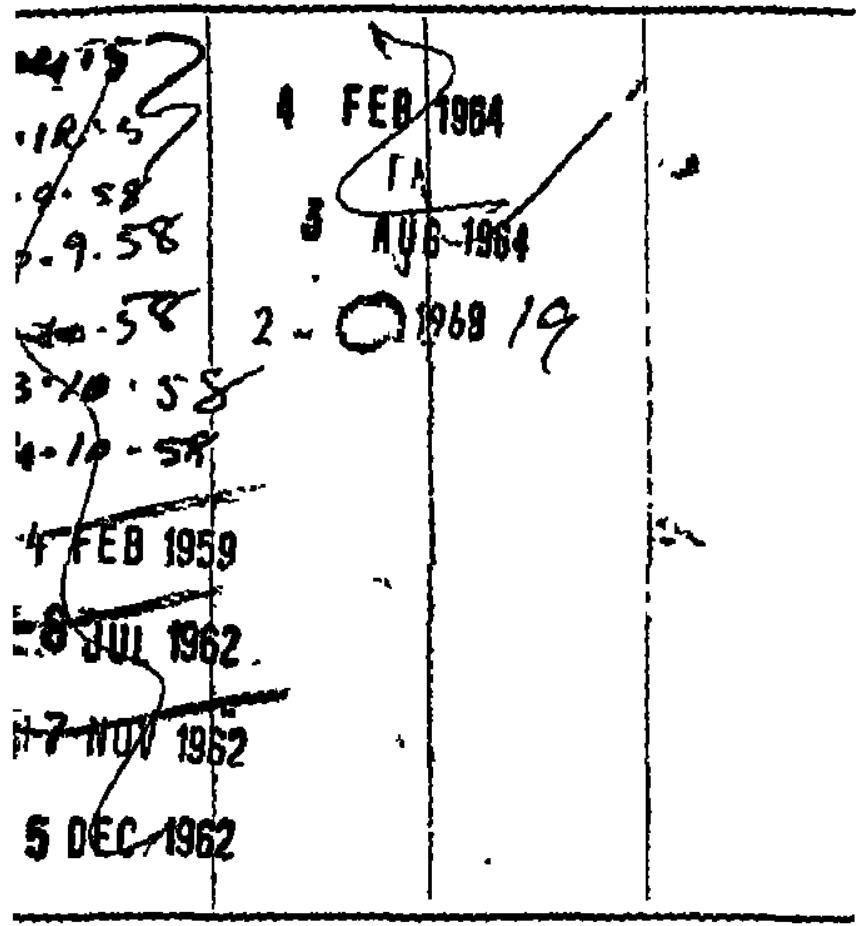

6/21/48 P] 8.2.48 406/109-4-4.6.48 3000 

Text books are issued for one day and the
general books tor 7 days excludipg the days of 1ssue and return

Books should be returned within the per tod for which they are issued as otherwise, $-11 /$ - per er 18 liable to a fine of annas and gener? day fur Texr Books kept overtione BE HELD STRICTS! BORROWERS WILL BE HELD SA DAME LY RESPONSIBLE FOR AHILE IN THEIR DONE TO THE BOOKS BOOK IS IN A POSSESSION. IF TH N BORROWED, DAMAGED STATE THE FACT SHOU THE LIBRARIAN. THE NOIICE OE THE LIBRARIAN.

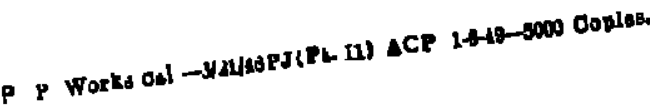

